\title{
Key role for VSMCs in vascular remodeling and calcification
}

Citation for published version (APA):

Jaminon, A. M. G. (2020). Key role for VSMCs in vascular remodeling and calcification. [Doctoral Thesis, Maastricht University]. Maastricht University. https://doi.org/10.26481/dis.20201002aj

Document status and date:

Published: 01/01/2020

DOI:

10.26481/dis.20201002aj

Document Version:

Publisher's PDF, also known as Version of record

\section{Please check the document version of this publication:}

- A submitted manuscript is the version of the article upon submission and before peer-review. There can be important differences between the submitted version and the official published version of record.

People interested in the research are advised to contact the author for the final version of the publication, or visit the DOI to the publisher's website.

- The final author version and the galley proof are versions of the publication after peer review.

- The final published version features the final layout of the paper including the volume, issue and page numbers.

Link to publication

\footnotetext{
General rights rights.

- You may freely distribute the URL identifying the publication in the public portal. please follow below link for the End User Agreement:

www.umlib.nl/taverne-license

Take down policy

If you believe that this document breaches copyright please contact us at:

repository@maastrichtuniversity.nl

providing details and we will investigate your claim.
}

Copyright and moral rights for the publications made accessible in the public portal are retained by the authors and/or other copyright owners and it is a condition of accessing publications that users recognise and abide by the legal requirements associated with these

- Users may download and print one copy of any publication from the public portal for the purpose of private study or research.

- You may not further distribute the material or use it for any profit-making activity or commercial gain

If the publication is distributed under the terms of Article $25 \mathrm{fa}$ of the Dutch Copyright Act, indicated by the "Taverne" license above, 


\section{Key role for VSMCs in vascular remodeling and calcification}




\section{Colofon}

(C)Armand Jaminon, Maastricht 2020

Cover design by Armand Jaminon, image provided by Chiara Recarti Printed by Ipskamp Printing, Enschede ISBN: 978-94-6421-008-8

All rights reserved. No part of this thesis may be reproduced, stored in a retrieved system or transmitted in any form or by any means, without the permission in writing from the author, or, when appropriate, of the publisher of the publication. 


\title{
Key role for VSMCs in vascular remodeling and calcification
}

\author{
PROEFSCHRIFT \\ Ter verkrijging van de graad doctor aan de Universiteit Maastricht, \\ Op gezag van de Rector Magnificus, Prof. dr. R.M. Letschert \\ Volgens het besluit van het College van Decanen, \\ In het openbaar te verdedigen op \\ Vrijdag 2 oktober 2020 om 12:00 uur
}

Door

Armand Mario Gerard Jaminon

Geboren op 21 november 1989 te Heerlen 


\section{Promotoren}

Prof. dr. L.J. Schurgers

Prof. dr. C.P.M. Reutelingsperger

\section{Beoordelingscommissie}

Prof. dr. P. de Leeuw (voorzitter)

Prof. dr. C Shanahan (King's College London)

Prof dr. J. Beulens (Amsterdam UMC)

Prof. dr. M.E. Kooi

Dr. M. Blankesteijn 
Voor mijn vrouw Famke, voor haar onvoorwaardelijke steun 



\section{Content}

$\begin{array}{lll}\text { Chapter } 1 \text { General introduction } & 9\end{array}$

$\begin{array}{lll}\text { Chapter } 2 & \text { The Role of Vascular Smooth Muscle Cells in Arterial } & 19\end{array}$

Remodeling: Focus on Calcification-Related Processes

Chapter 3 The role of medial vascular calcification on the development 49 and progression of atherosclerosis

Chapter 4 Medial vascular smooth muscle cell apoptosis accelerates 63 atherogenesis and promotes features of a vulnerable plaque

Chapter 5 Use of primary vascular smooth muscle cells to predict 81 vascular calcification potential of patients: Development of a BioHybrid Assay

Chapter 6 Matrix Gla protein is an independent predictor of both 97 intimal and medial vascular calcification in chronic kidney disease

Chapter 7 General discussion

Appendices Summary

Nederlandse samenvatting

Valorization

Curriculum vitae

List of publications

Dankwoord 


\section{Chapter 1}

General introduction 
Chapter 1

\section{Cardiovascular disease}

The world health organization has reported that cardiovascular disease (CVD) is the number one cause of mortality worldwide ${ }^{1}$. In 2017 an estimated 17.8 million people died from CVD which represents $32 \%$ of all global deaths. The majority of CVD related deaths is caused by myocardial infarction and ischemic stroke ${ }^{2}$. Additionally, most CVDs occur in low- to middle-income countries, where early detection and treatment is not included in primary healthcare programs.

One of the key players in CVD development is arterial remodeling. Continues stress on the vessel wall results in arterial remodeling initiating mechanisms to repair the vessel wall. Long-term stress induces structural and biological changes that alter characteristics of the vessel wall, leading to chronic vascular remodeling, pathological adaptation and vascular stiffness. This results in increased vessel wall thickness due to hyperplasia and phenotypic switching of vascular smooth muscle cells (VSMCs). Increased arterial stiffness is often accompanied with presence of vascular calcification. In the last decade, vascular calcification was added to the growing list of risk factors for CVD mortality and morbidity, however the precise role of vascular calcification in CVD still needs to be unraveled ${ }^{3,4}$.

\section{Arterial remodeling}

Arterial remodeling reflects adaptation of the vessel wall to biochemical and biomechanical stimuli ${ }^{5,6}$. In the vasculature, there are two types of remodeling, namely outward and inward remodeling. Both types of remodeling coincide with hypertrophy (thickening) or hypotrophy (thinning) of the vessel wall. VSMCs are key in the development of arterial remodeling. In the vessel wall, VSMCs are connected to a fenestrated network of elastin and collagen fibres. The capacity of the vessel wall to elastically distend is important to accommodate the volume ejected with each heartbeat and to limit peripheral pressure pulsations. The active tone and spatial arrangement of VSMCs may influence mechanical load on extracellular matrix (ECM) components and therefore, modulate vessel diameter and stiffness ${ }^{7}$.

Chronic exposure to high blood pressure increases tensile stress. VSMCs respond by proliferation, resulting in hyperplasia and thickening of the vascular wall. Activation of MMPs facilitate breakdown of elastin and synthetic VSMCs produce collagen, attempting to preserve stiffness homeostasis ${ }^{8}$. Additional ECM disturbances, such as presence of vascular calcification, have further impact on remodelling and stiffening of the medial vascular layer ${ }^{9,10}$. Vascular calcification is defined as pathological mineral deposits in the vessel wall. There are two distinct sites of vascular calcification: medial and intimal vascular calcification, referring to the layer of the vessel wall were calcification is found ${ }^{11}$. Arterial remodeling is complex and this thesis aims to address unresolved issues in the interplay between vascular stiffening, vascular calcification and atherosclerosis (Figure 1). 

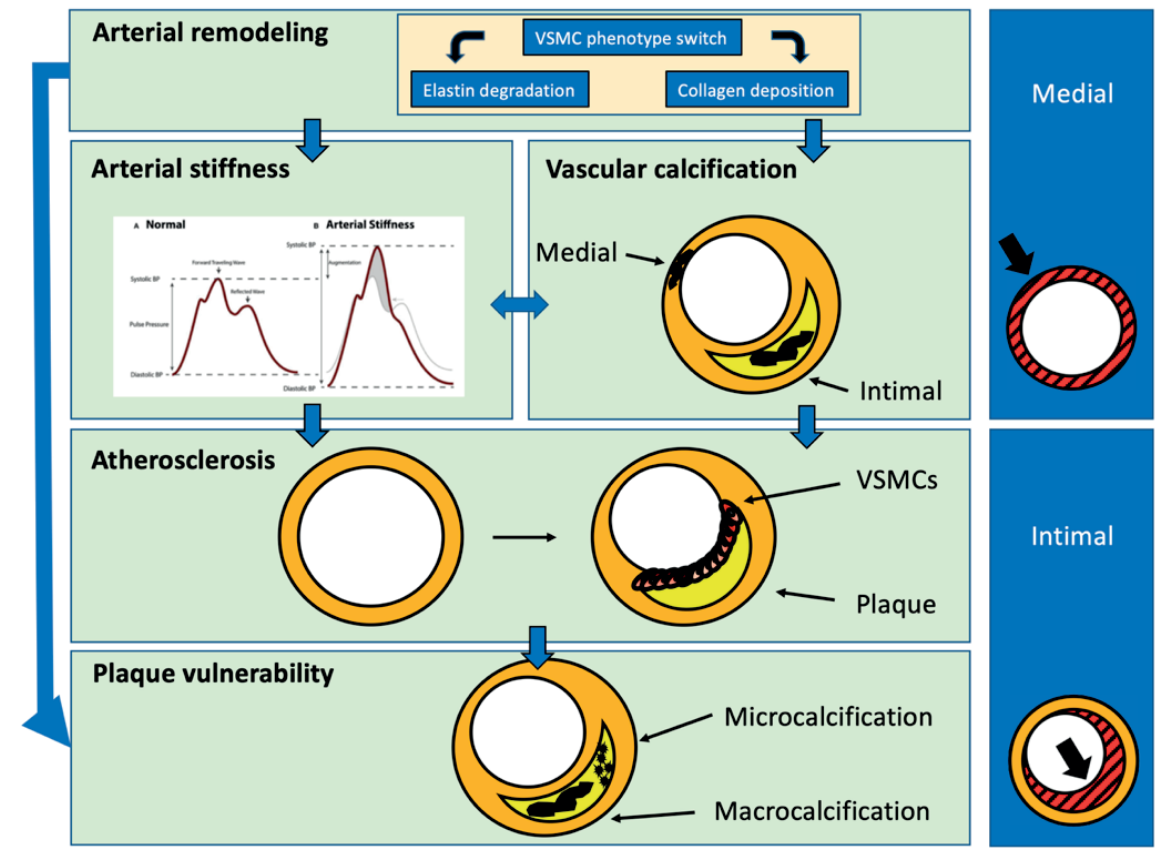

Figure 1. The interplay between arterial remodeling, vascular stiffening, vascular calcification and atherosclerosis. Arterial remodeling processes are initiated by VSMCs who undergo a phenotype switch under stress stimuli, leading to collagen deposition and elastin degradation. The increased collagen deposition combined with lower elasticity lead to arterial stiffness and can form a nidus for the development of vascular calcification. Vascular calcification can be found at two distinct sites: medial and intimal. Both arterial stiffness and medial calcification can initiate atherosclerosis due to flow disturbances and shear stress. Once plaques mature they develop into vulnerable plaques that are prone to rupture. Inside atherosclerotic plaques VSMC phenotype switching plays an important role. Here VSMCs can induce calcification that starts as microcalcification and can develop into large macrocalcifications.

\section{Atherosclerosis}

Atherosclerosis is the pathological build-up of plaques in the intimal space thereby thickening the vessel wall and narrowing the lumen. Atherosclerosis is considered an inflammatory disease that is ignited by endothelial stress and lipid accumulation with subsequent leucocyte infiltration ${ }^{12,13}$. Atherosclerosis development is, in part, driven by factors that are present in the blood compartment. Lipids, high glucose and disturbed hemodynamics, e.g. caused by high blood pressure, can activate the endothelium initiating plaque development ${ }^{14}$. More recently, local vascular processes are considered to be important in the development of atherosclerosis ${ }^{15,16}$. Lineage tracing studies in mice revealed that many cells present in the atherosclerotic plaque are derived from VSMC precursors ${ }^{17}$. Moreover, VSMC traces are found in atherosclerotic lesions which often co-localise with macrophage markers ${ }^{18}$. This implies that cells in the intima are recruited from the vessel media instead of infiltrating 
mesenchymal cells from the blood compartment ${ }^{18,19}$. VSMCs exert multiple roles and functions in atherosclerosis. During plaque development, VSMCs can migrate and form a fibrous cap in which they excrete extracellular matrix proteins (ECMs) that stabilize the plaque. Oppositely, intra-plaque VSMCs can change toward a macrophage-like phenotype 19,20. Furthermore, VSMCs undergoing apoptosis with diminished efferocytosis form a nidus for calcification ${ }^{21,22}$.

\section{Vascular calcification}

Intimal calcification often coincides with atherosclerosis as both are pathological processes affecting the intimal layer of the vessel wall and initiate luminal narrowing. Intimal calcification refers to build-up of calcified areas within atherosclerotic lesions. Intimal calcification can exist of two forms: micro- and macrocalcification. Intimal calcification is used as a measure of atherosclerotic burden 23 and large areas of calcification $(>50 \mu \mathrm{m})$ are considered macrocalcification. Macrocalcification has been recently associated with increased plaque stability ${ }^{24}$. The plaque stabilising effect of calcification was further adopted since statins, used to lower plasma lipids, have plaque stabilising effects and concomitant increase intimal calcification ${ }^{25-27}$. On the other hand, microcalcification $(<50 \mu \mathrm{m})$ has been shown to decrease plaque stability and thereby favour thrombus formation ${ }^{28}$. Clinically, the distinction between micro- and macrocalcification is not possible due to a lack of resolution of computed tomography (CT), being $200 \mu \mathrm{m}{ }^{29}$. Distinguishing vascular calcification based on size is limited and not telling the complete story as density and location of calcification also contribute to the pathogenesis of cardiovascular disease. Coronary artery calcium (CAC) volume is associated with CVD risk whereas CAC density is inversely correlated with CVD risk ${ }^{30}$. Additionally, the location where calcium deposits reside inside atherosclerotic plaques determines its vulnerability to rupture. Calcium deposits that are located in the necrotic core do not seem to have significant impact on plaque stability whereas calcium deposits that are in the fibrous cap region may lead to plaque rupture ${ }^{31}$.

Medial calcification, also known as Mönckebergsclerosis, is associated with ageing and is regularly found in peripheral arteries ${ }^{32,33}$. Medial calcification can be found along the entire vascular tree and is often found in patients suffering from diabetes and/or chronic kidney disease (CKD) ${ }^{34,35}$. Medial calcification is an important confounding factor in the development of vascular stiffening and hypertension, two clinical entities that affect the physiological condition of the vessel wall ${ }^{36,37}$. Interestingly, early literature reports that medial remodeling or calcification precedes at sites of atherosclerosis ${ }^{38}$. More recent data suggest an interaction between both medial and intimal calcification, in which both can give impact one another ${ }^{39,40}$. There are multiple factors and pathways leading to vascular calcification (Figure 2). Below I will summarize the most important. 


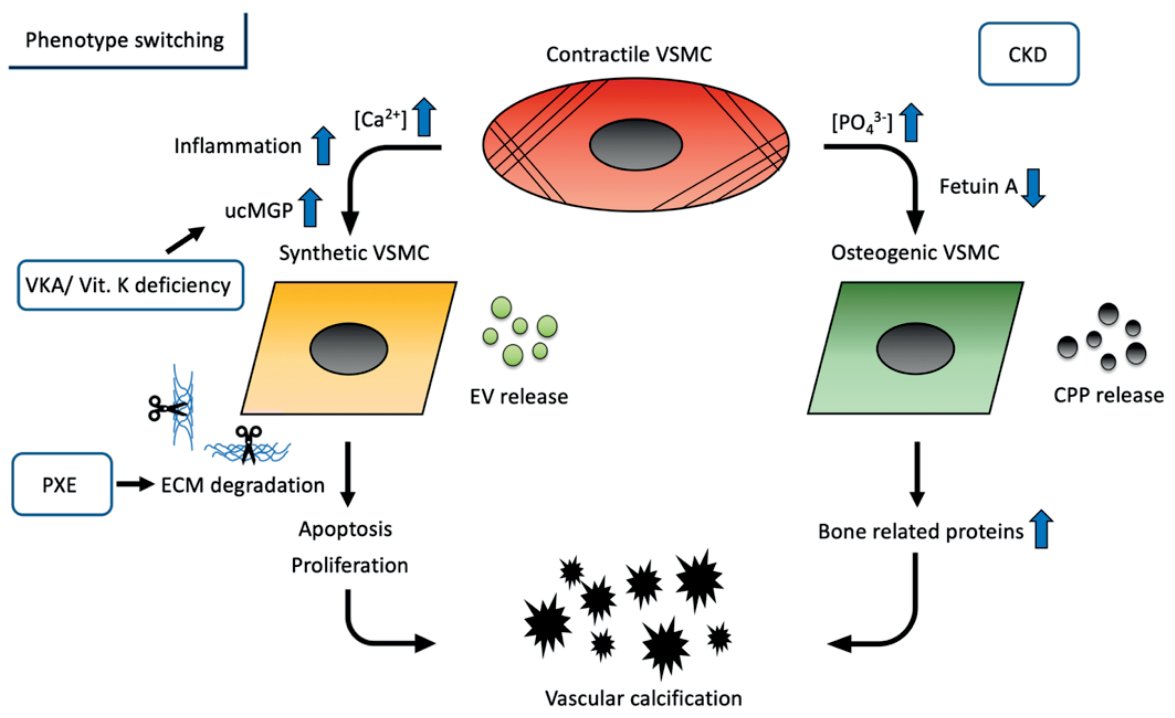

Figure 2. VSMC phenotype switching. Under physiological condition VSMCs have a contractile phenotype which is necessary to cope with alterations in blood pressure. Upon pathological signals VSMCs respond and initiate a phenotype switch. VSMCs can switch to a synthetic phenotype under increased calcium and inflammation. Vitamin K deficiency, often found in patients on Vitamin K antagonist, induces a VSMC synthetic switch. Synthetic VSMCs are known to induce apoptosis, proliferation, extracellular vesicle release and affect the extracellular matrix. VSMC osteogenic phenotype switch is induced by increased phosphate and lower Fetuin A plasma levels. Osteogenic VSMCs are known to secrete calciprotein particles and increase bone related protein expression. Both synthetic and osteogenic VSMCs are known to cause vascular calcification.

\section{VSMC phenotype switching}

VSMCs display phenotypic plasticity during normal development, disease and repair of the vasculature. Under pathological signals such as inflammation or mineral disbalance, VSMCs undergo phenotypic switching. This VSMC phenotype switching is characterized by increased proliferation and migration ${ }^{41}$ and extracellular vesicle (EV) release ${ }^{42}$. High levels of calcium and phosphate drive VSMCs towards an osteogenic phenotype which express bone related proteins and deposit mineralized bone-like matrix 43.

Tissue damage and inflammation of the vessel wall can initiate calcification by the induction of VSMC apoptosis. Apoptosis is considered to be a central aspect of vascular calcification, due to the shedding of apoptotic bodies that can form a nidus for calcification ${ }^{44,45}$. Moreover, synthetic VSMCs are able to shed EVs that can serve as nidus for calcification ${ }^{42,46}$. Furthermore, in vitro experiments have demonstrated that apoptosis increases calcification whereas inhibition of apoptosis decreases vascular calcification $^{21}$. 


\section{Loss of calcification inhibitors}

In order to prevent spontaneous calcification, inhibitors of calcification are present in both the circulation and in the vasculature. Fetuin-A and matrix Gla protein (MGP) are such proteins with strong ion binding capacity ${ }^{47,48}$. Fetuin-A is a plasma protein, mainly synthesized in liver and plays a critical role in the stabilization of proteinmineral complexes, also termed calciprotein particles (CPPs). The formation of CPPs prevents pathological calcium and phosphate deposition in tissue if they are cleared from the circulation. CPP clearance is attributed to liver Kupffer cells and if not performed correctly leads to particle-induced inflammation and vascular calcification ${ }^{49}$.

MGP is a vitamin $\mathrm{K}$-dependent protein that requires vitamin $\mathrm{K}$ to become biologically active ${ }^{48}$. In mice, MGP knock-out ${ }^{50}$ or drug induced knock-down of active MGP ${ }^{10,51}$ results in both intimal and medial vascular calcification. Additionally, in patients, usage of vitamin $\mathrm{K}$ antagonists is found to induce vascular calcification ${ }^{52-54}$.

Other well-known calcification inhibitors are osteoprotegerin (OPG), pyrophosphate (ENPP1) and osteopontin (OPN). Clinically, high levels of OPG and lower levels of ENPP1 are associated with atherosclerosis and vascular calcification ${ }^{55}$. Furthermore, OPN is found in mineralized tissues such as bone and teeth but also in calcified plaques ${ }^{56}$.

\section{Disease-related calcification}

Many diseases are associated with a higher risk for vascular calcification. Type 2 Diabetes Mellitus (T2DM) is associated with increased prevalence of CVD and mortality. T2DM patients appear to have extensive medial calcification which is an independent predictor of cardiovascular mortality ${ }^{57}$.

CKD patients suffer from hyperphosphatemia which is, on its own, a risk factor for increased mortality ${ }^{58}$. Additionally, high phosphate levels are associated with severe progression of vascular calcification ${ }^{59}$. The uremic environment that CKD patients display evokes VSMC osteogenic phenotype switching that results in mineralization of surrounding tissue ${ }^{33,60}$.

The interaction between inflammation and vascular calcification is not completely understood. Vascular calcification can be found in atherosclerosis, which is considered an inflammatory disease, and many inflammatory markers are associated with vascular calcification ${ }^{61}$. On the other hand, medial vascular calcification is less associated with inflammation. Chronic inflammation is known to be associated with atherosclerotic calcification in mice ${ }^{62}$ and has been confirmed by ${ }^{18} \mathrm{~F}$-FDG positron emission tomography with subsequent CT scanning in patients ${ }^{63}$.

Pseudoxanthoma elasticum (PXE) is a monogenetic disorder caused by a mutation in the ATP binding cassette subfamily $\mathrm{C}$ member 6 (ABCC6) gene. PXE is characterized by dystrophic mineralization of soft connective structures, primarily elastic fibers. PXE manifests in several organs such as the skin, eye and heart ${ }^{64}$. Additionally, CVD is often encountered, primarily reflected by mineralization of mid-sized arteries in 
the lower extremities. The onset of PXE is usually rather late and slowly progressing, which clinically manifests as mineralization of the Bruchs' membrane in the eye and or elastic fibers in the arterial blood vessels causing increased morbidity with occasional mortality ${ }^{65}$.

\section{Outline of this thesis}

The research described in this thesis addresses arterial remodeling: the interaction between vascular stiffness and vascular calcification both in relation to atherosclerosis. In more detail, I investigated the role of vascular smooth muscle phenotypic switching in vascular remodeling in relation to calcification.

Chapter 2 elaborates on the interactions between vascular remodeling, vascular stiffness and vascular calcification. In this chapter, we discuss the role and phenotypic plasticity of VSMCs in vascular remodeling. We review in detail the latest clinical techniques of measuring vascular remodeling.

Chapter 3 focusses on the role of VSMC calcification in the tunica media in relation to the initiation and development of atherosclerosis. To address this, I used DBA/2 mice that are prone to develop medial vascular calcification. Using a viral LDLr knock-down this allowed me to study the role of medial calcification on the development of atherosclerosis.

Chapter 4 focusses on the role of VSMC apoptosis in the tunica media in relation to the initiation and development of atherosclerosis. I have employed an $\mathrm{ApoE}^{-}$ - mouse model that has knocked in the human diphtheria toxin receptor specifically in VSMCs which allowed me to reduce VSMC number in the vessel wall prior to initiation of atherosclerosis. In Chapter 4, I present data on the role and contribution of medial VSMCs in the development of atherosclerosis.

In chapter 5 I describe a novel approach to measure VSMC calcification. We reason that the biomarker for vascular calcification is not a single one but rather the collection of all plasma components. This paper describes the new cell-based assay for measuring the composite biomarker for vascular calcification.

Chapter 6 elaborates on this first clinical study by focusing on the role of vitamin K-dependent MGP in vascular calcification and whether MGP can be used as biomarker to predict presence of vascular calcification. We measured MGP by different means, ranging from genomic level (MGP SNPs) to expression (RNA levels) and protein excretion (immunohistochemical staining and plasma levels).

In chapter 7 key findings are summarized and the results from this thesis are discussed, additionally, future research directions are provided. 


\section{References}

1. World Health Organization. Global Health Estimates 2016: Death by Cause, Age, Sex, by Country and by Region, 2000-2016. 1-65 (2018).

2. Roth, G. A. et al. Global, regional, and national age-sex-specific mortality for 282 causes of death in 195 countries and territories, 1980-2017: a systematic analysis for the Global Burden of Disease Study 2017. The Lancet 392, 1736-1788 (2018).

3. Sage, A. P., Tintut, Y. \& Demer, L. L. Regulatory mechanisms in vascular calcification. Nature Reviews Cardiology 2014 11:7 7, 528-536 (2010).

4. Rennenberg, R. J. M. W. et al. Vascular calcifications as a marker of increased cardiovascular risk: a meta-analysis. Vasc Health Risk Manag 5, 185-197 (2009).

5. O'Rourke, M. F. \& Nichols, W. W. Aortic Diameter, Aortic Stiffness, and Wave Reflection Increase With Age and Isolated Systolic Hypertension. Hypertension 45, 652-658 (2005).

6. van Varik, B. J. et al. Mechanisms of arterial remodeling: lessons from genetic diseases. Front Genet 3, 290 (2012).

7. Reesink, K. D. \& Spronck, B. Constitutive interpretation of arterial stiffness in clinical studies: a methodological review. Am. J. Physiol. Heart Circ. Physiol. 316, H693-H709 (2019).

8. Humphrey, J. D., Dufresne, E. R. \& Schwartz, M. A. Mechanotransduction and extracellular matrix homeostasis. Nat. Rev. Mol. Cell Biol. 15, 802-812 (2014).

9. Sekikawa, A. et al. Aortic stiffness and calcification in men in a population-based international study. Atherosclerosis 222, 473-477 (2012).

10. Kruger, T. et al. Warfarin Induces Cardiovascular Damage in Mice. Arteriosclerosis, Thrombosis, and Vascular Biology 33, 2618-2624 (2013).

11. Willems, B. A. G., Vermeer, C., Reutelingsperger, C. P. M. \& Schurgers, L. J. The realm of vitamin K dependent proteins: Shifting from coagulation toward calcification. Mol Nutr Food Res 58, 1620 1635 (2014).

12. Hopkins, P. N. Molecular Biology of Atherosclerosis. Physiological Reviews 93, 1317-1542 (2013).

13. Bennett, M. R., Sinha, S. \& Owens, G. K. Vascular Smooth Muscle Cells in Atherosclerosis. Circ. Res. 118, 692-702 (2016).

14. Vaidya, D. et al. Association of endothelial and oxidative stress with metabolic syndrome and subclinical atherosclerosis: multi-ethnic study of atherosclerosis. European Journal of Clinical Nutrition 65, 818-825 (2011).

15. Chatrou, M. L. L. et al. Intra-Section Analysis of Human Coronary Arteries Reveals a Potential Role for Micro-Calcifications in Macrophage Recruitment in the Early Stage of Atherosclerosis. PLoS ONE 10, e0142335 (2015).

16. Hao, Y.-M. et al. Endothelial to mesenchymal transition in atherosclerotic vascular remodeling. Clin. Chim. Acta 490, 34-38 (2019).

17. Liu, M. \& Gomez, D. Smooth Muscle Cell Phenotypic Diversity. Arteriosclerosis, Thrombosis, and Vascular Biology 75, ATVBAHA119312131 (2019).

18. Feil, S. et al. Transdifferentiation of vascular smooth muscle cells to macrophage-like cells during atherogenesis. Circ. Res. 115, 662-667 (2014).

19. Dzau, V. J., Braun-Dullaeus, R. C. \& Sedding, D. G. Vascular proliferation and atherosclerosis: new perspectives and therapeutic strategies. Nat. Med. 8, 1249-1256 (2002).

20. Allahverdian, S., Chehroudi, A. C., McManus, B. M., Abraham, T. \& Francis, G. A. Contribution of intimal smooth muscle cells to cholesterol accumulation and macrophage-like cells in human atherosclerosis. Circulation 129, 1551-1559 (2014).

21. Proudfoot, D. et al. Apoptosis regulates human vascular calcification in vitro: evidence for initiation of vascular calcification by apoptotic bodies. Circ. Res. 87, 1055-1062 (2000).

22. Clarke, M. C. H. et al. Apoptosis of vascular smooth muscle cells induces features of plaque vulnerability in atherosclerosis. Nat. Med. 12, 1075-1080 (2006).

23. Greenland, P. et al. ACCF/AHA 2007 clinical expert consensus document on coronary artery calcium scoring by computed tomography in global cardiovascular risk assessment and in evaluation 
of patients with chest pain: a report of the American College of Cardiology Foundation Clinical Expert Consensus Task Force (ACCF/AHA Writing Committee to Update the 2000 Expert Consensus Document on Electron Beam Computed Tomography). in 115, 402-426 (2007).

24. Shioi, A. \& Ikari, Y. Plaque Calcification During Atherosclerosis Progression and Regression. $J$. Atheroscler. Thromb. 25, 294-303 (2018).

25. Terry, J. G. et al. Effect of simvastatin $(80 \mathrm{mg})$ on coronary and abdominal aortic arterial calcium (from the coronary artery calcification treatment with zocor [CATZ] study). Am. J. Cardiol. 99, 1714-1717 (2007).

26. Räber, L. et al. Effect of high-intensity statin therapy on atherosclerosis in non-infarct-related coronary arteries (IBIS-4): a serial intravascular ultrasonography study. Eur. Heart J. 36, 490-500 (2015).

27. Chen, Z. et al. Does statins promote vascular calcification in chronic kidney disease? Eur. J. Clin. Invest. 47, 137-148 (2017).

28. Irkle, A. et al. Identifying active vascular microcalcification by $<$ sup $>18</$ sup $>$ F-sodium fluoride positron emission tomography. Nature Communications 2015 6 6, 7495 (2015).

29. Stary, H. C. Natural history of calcium deposits in atherosclerosis progression and regression. $Z$ Kardiol 89 Suppl 2, 28-35 (2000).

30. Criqui, M. H. et al. Coronary Artery Calcium Volume and Density: Potential Interactions and Overall Predictive Value: The Multi-Ethnic Study of Atherosclerosis. JACC Cardiovasc Imaging 10, 845854 (2017).

31. Li, Z.-Y. et al. Does calcium deposition play a role in the stability of atheroma? Location may be the key. Cerebrovasc. Dis. 24, 452-459 (2007).

32. Covic, A. et al. Vascular calcification in chronic kidney disease. Clin. Sci. 119, 111-121 (2010).

33. Dai, L., Qureshi, A. R., Witasp, A., Lindholm, B. \& Stenvinkel, P. Early Vascular Ageing and Cellular Senescence in Chronic Kidney Disease. Comput Struct Biotechnol J 17, 721-729 (2019).

34. Edmonds, M. E. Medial arterial calcification and diabetes mellitus. Z Kardiol 89 Suppl 2, 101-104 (2000).

35. Garimella, P. S. \& Sarnak, M. J. Cardiovascular disease in CKD in 2012: moving forward, slowly but surely. Nat Rev Nephrol 9, 69-70 (2013).

36. Blacher, J., Guérin, A. P., Pannier, B., Marchais, S. J. \& London, G. M. Arterial calcifications, arterial stiffness, and cardiovascular risk in end-stage renal disease. Hypertension 38, 938-942 (2001).

37. Neven, E. \& D'Haese, P. C. Vascular calcification in chronic renal failure: what have we learned from animal studies? Circ. Res. 108, 249-264 (2011).

38. Blumenthal, H. Calcification of the media of the human aorta and its relation to intimal arteriosclerosis, ageing and disease. 1-23 (2007).

39. Giachelli, C. M. Vascular calcification mechanisms. J. Am. Soc. Nephrol. 15, 2959-2964 (2004).

40. Clarke, M. C. H. et al. Chronic apoptosis of vascular smooth muscle cells accelerates atherosclerosis and promotes calcification and medial degeneration. Circ. Res. 102, 1529-1538 (2008).

41. Allahverdian, S., Chaabane, C., Boukais, K., Francis, G. A. \& Bochaton-Piallat, M.-L. Smooth muscle cell fate and plasticity in atherosclerosis. Cardiovasc. Res. 114, 540-550 (2018).

42. Kapustin, A. N. et al. Vascular smooth muscle cell calcification is mediated by regulated exosome secretion. Circ. Res. 116, 1312-1323 (2015).

43. Goettsch, C. et al. Sortilin mediates vascular calcification via its recruitment into extracellular vesicles. J. Clin. Invest. 126, 1323-1336 (2016).

44. Demer, L. L. \& Tintut, Y. Vascular calcification: pathobiology of a multifaceted disease. Circulation 117, 2938-2948 (2008).

45. Shroff, R. C. et al. Dialysis accelerates medial vascular calcification in part by triggering smooth muscle cell apoptosis. Circulation 118, 1748-1757 (2008).

46. Schlieper, G., Schurgers, L., Brandenburg, V., Reutelingsperger, C. \& Floege, J. Vascular calcification in chronic kidney disease: an update. Nephrol. Dial. Transplant. 31, 31-39 (2016). 


\section{Chapter 1}

47.

48.

54.

Jahnen-Dechent, W., Heiss, A., Schäfer, C. \& Ketteler, M. Fetuin-A regulation of calcified matrix metabolism. Circ. Res. 108, 1494-1509 (2011).

Schurgers, L. J., Uitto, J. \& Reutelingsperger, C. P. Vitamin K-dependent carboxylation of matrix Gla-protein: a crucial switch to control ectopic mineralization. Trends Mol Med 19, 217-226 (2013). Köppert, S. et al. Cellular Clearance and Biological Activity of Calciprotein Particles Depend on Their Maturation State and Crystallinity. Front Immunol 9, 1991 (2018).

Luo, G. et al. Spontaneous calcification of arteries and cartilage in mice lacking matrix GLA protein. Nature 386, 78-81 (1997).

Schurgers, L. J. et al. Vitamin K-antagonists accelerate atherosclerotic calcification and induce a vulnerable plaque phenotype. PLOS ONE 7, e43229 (2012).

Weijs, B. et al. Patients using vitamin K antagonists show increased levels of coronary calcification: an observational study in low-risk atrial fibrillation patients. Eur. Heart J. 32, 2555-2562 (2011).

Chatrou, M. L. L., Winckers, K., Hackeng, T. M., Reutelingsperger, C. P. \& Schurgers, L. J. Vascular calcification: The price to pay for anticoagulation therapy with vitamin $\mathrm{K}$-antagonists. YBLRE 26, 155-166 (2012).

4. Caluwé, R., Pyfferoen, L., De Boeck, K. \& De Vriese, A. S. The effects of vitamin K supplementation and vitamin $\mathrm{K}$ antagonists on progression of vascular calcification: ongoing randomized controlled trials. Clin Kidney J 9, 273-279 (2016).

5azama, J. J. et al. Increased circulating levels of osteoclastogenesis inhibitory factor (osteoprotegerin) in patients with chronic renal failure. Am. J. Kidney Dis. 39, 525-532 (2002).

Giachelli, C. M., Speer, M. Y., Li, X., Rajachar, R. M. \& Yang, H. Regulation of vascular calcification: roles of phosphate and osteopontin. Circ. Res. 96, 717-722 (2005).

Chen, N. X. \& Moe, S. M. Arterial calcification in diabetes. Curr. Diab. Rep. 3, 28-32 (2003).

Bai, W., Li, J. \& Liu, J. Serum phosphorus, cardiovascular and all-cause mortality in the general population: A meta-analysis. Clin. Chim. Acta 461, 76-82 (2016).

Kovesdy, C. P. \& Kalantar-Zadeh, K. Serum phosphorus and the risk of progression of chronic kidney disease. Nephrol. Dial. Transplant. 22, 3679-3680 (2007).

Voelkl, J. et al. Signaling pathways involved in vascular smooth muscle cell calcification during hyperphosphatemia. Cell. Mol. Life Sci. 76, 2077-2091 (2019).

Demer, L. L. \& Tintut, Y. Inflammatory, metabolic, and genetic mechanisms of vascular calcification. Arteriosclerosis, Thrombosis, and Vascular Biology 34, 715-723 (2014).

Aikawa, E. et al. Osteogenesis associates with inflammation in early-stage atherosclerosis evaluated by molecular imaging in vivo. Circulation 116, 2841-2850 (2007).

63. Abdelbaky, A. et al. Focal arterial inflammation precedes subsequent calcification in the same location: a longitudinal FDG-PET/CT study. Circ Cardiovasc Imaging 6, 747-754 (2013).

Uitto, J., Li, Q. \& Jiang, Q. Pseudoxanthoma elasticum: molecular genetics and putative pathomechanisms. J. Invest. Dermatol. 130, 661-670 (2010).

Uitto, J., Li, Q., van de Wetering, K., Váradi, A. \& Terry, S. F. Insights into Pathomechanisms and Treatment Development in Heritable Ectopic Mineralization Disorders: Summary of the PXE International Biennial Research Symposium-2016. J. Invest. Dermatol. 137, 790-795 (2017). 


\section{Chapter 2}

The Role of Vascular Smooth Muscle Cells in Arterial Remodeling: Focus on Calcification-Related Processes

A.M.G. Jaminon, K. Reesink, A.A. Kroon and L.J. Schurgers

Int. J. Mol. Sci. 2019, 20, 5694 


\title{
Chapter 2
}

\begin{abstract}
Arterial remodeling refers to the structural and functional changes of the vessel wall that occur in response to disease, injury, or aging. Vascular smooth muscle cells (VSMC) play a pivotal role in regulating the remodeling processes of the vessel wall. Phenotypic switching of VSMC involves oxidative stress-induced extracellular vesicle release, driving calcification processes. The VSMC phenotype is relevant to plaque initiation, development and stability, whereas, in the media, the VSMC phenotype is important in maintaining tissue elasticity, wall stress homeostasis and vessel stiffness. Clinically, assessment of arterial remodeling is a challenge; particularly distinguishing intimal and medial involvement, and their contributions to vessel wall remodeling. The limitations pertain to imaging resolution and sensitivity, so methodological development is focused on improving those. Moreover, the integration of data across the microscopic (i.e., cell-tissue) and macroscopic (i.e., vessel-system) scale for correct interpretation is innately challenging, because of the multiple biophysical and biochemical factors involved. In the present review, we describe the arterial remodeling processes that govern arterial stiffening, atherosclerosis and calcification, with a particular focus on VSMC phenotypic switching. Additionally, we review clinically applicable methodologies to assess arterial remodeling and the latest developments in these, seeking to unravel the ubiquitous corroborator of vascular pathology that calcification appears to be.
\end{abstract}




\section{Introduction}

The vessel wall consists of multiple cells, including endothelial cells (ECs), vascular smooth muscle cells (VSMCs), fibroblasts and pericytes, and structural extracellular matrix (ECM) components, such as elastin and collagen ${ }^{1}$. ECs form the innermost layer, being the barrier between the blood-carrying lumen and vessel wall. In the vessel wall, ECs are surrounded by the tunica media, consisting almost entirely of circumferentially oriented VSMCs. Medial VSMCs, facilitating vessel dilation and constriction, are surrounded by interconnected elastic lamellae ${ }^{2}$. The outer layer of the vessel wall is the adventitia and contains ECM, maintaining structural integrity under peak mechanical load, and fibroblasts and progenitor (mesenchymal stem cell-like, MSC-like) cells. The interplay of all components of the vessel wall is needed to preserve vascular health ${ }^{3-5}$.

The vessel wall is continuously exposed to biomechanical and biochemical stressors that elicit functional and adaptative responses. For instance, when blood flow is acutely increased, the increased wall shear stress (or friction) is sensed by ECs, which release relaxants. These relaxants (e.g., nitric oxide, NO) lower the active tone of VSMCs, leading to (flow-mediated) vessel dilation to counteract the initial increase in wall shear stress ${ }^{6}$. Long-term changes in wall shear stress provoke diameter adaptation ${ }^{7}$. Wall thickness changes are seen in response to increased wall stress (or wall tension), due to high blood pressure ${ }^{8}$. Such arterial remodeling responses shape early development of the macro-circulation, but are also affected by normal aging processes ${ }^{9}$. A multitude of biological processes contribute to pathological vascular remodeling, such as inflammation, oxidative stress, lipid accumulation, and degradation of the ECM ${ }^{10,11}$. Due to the heterogeneity of vascular remodeling, it is difficult to pin-point single biological processes responsible for vascular disease. Lessons from genetic disorders such as pseudoxanthoma elasticum (PXE), Marfan's syndrome and Keutel syndrome, have addressed the complexity of arterial remodeling ${ }^{12}$. A better understanding of the complexity of arterial remodeling will help unravel its role as a cause or consequence of cardiovascular disease (CVD).

VSMCs in the medial layer of the vessel wall play a key role in arterial remodeling. VSMCs are the most abundant cell type in the arterial vessel wall and are pivotal in maintaining vessel structure and function ${ }^{13}$. VSMCs are considered heterogeneous and display a high degree of plasticity ${ }^{5}$. Under physiological conditions, VSMCs have a contractile phenotype which facilitates the contraction and dilation of the vasculature, which, in smaller resistance arteries, is key to the regulation of blood flow. Upon biological stress signals or vascular injury, VSMCs respond by losing contractility markers and differentiate towards a synthetic VSMC phenotype. Synthetic VSMCs subsequently express proteins involved in proliferation and migration ${ }^{14}$. Contractile VSMCs may adapt to stress by differentiating towards synthetic VSMCs which are able to newly synthesize ECM components such as collagen. To remodel the ECM, synthetic VSMCs produce metalloproteinases (MMPs), such as collagenases and elastases, that allow them to migrate to sites of injury ${ }^{15-17}$. VSMC-mediated remodeling of ECM within the vessel wall may result in increased arterial stiffness ${ }^{13,18}$, contributing to systolic hypertension and altered hemodynamic conditions in end-organs such as the brain, kidneys and heart ${ }^{19}$.

VSMCs are involved in many vascular diseases, such as atherosclerosis and aneurysm formation. In all these vascular pathologies, vascular calcification is involved 
$5,20,21$. Vascular calcification is defined as the deposition of calcium crystals within the vessel wall, initiated as microcalcification and propagating towards macrocalcifications ${ }^{22}$, and, eventually, encroaching entire segments of the vasculature ${ }^{23}$. Vascular calcification is an active process with a key role for VSMCs, including apoptosis ${ }^{24}$, osteochondrogenic transdifferentiation ${ }^{25}$, extracellular vesicle release ${ }^{2}$, calcium overload ${ }^{26}$, and cellular senescence ${ }^{27}$ (Figure 1). The aim of the present review is to provide an overview of the pathways underlying arterial remodeling, with a particular focus on the role of VSMCs and calcification-related processes.

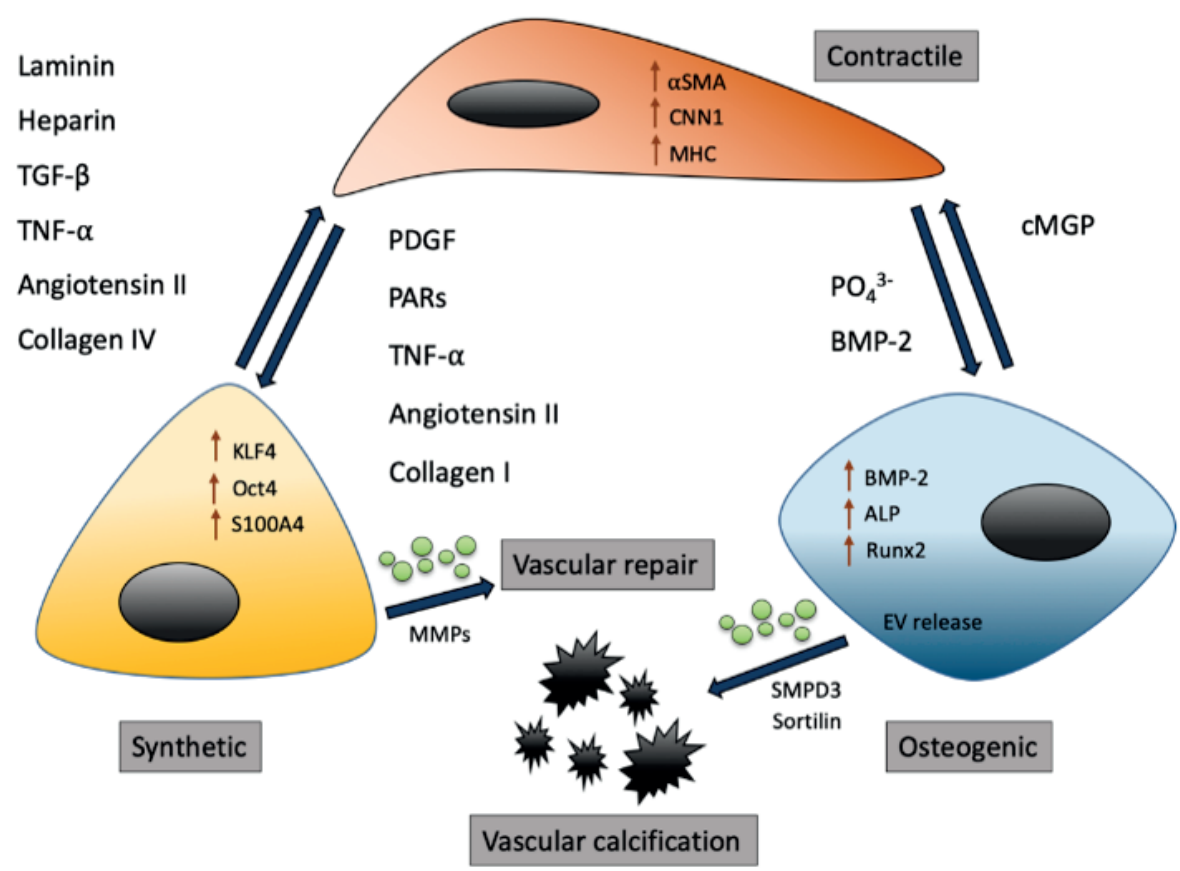




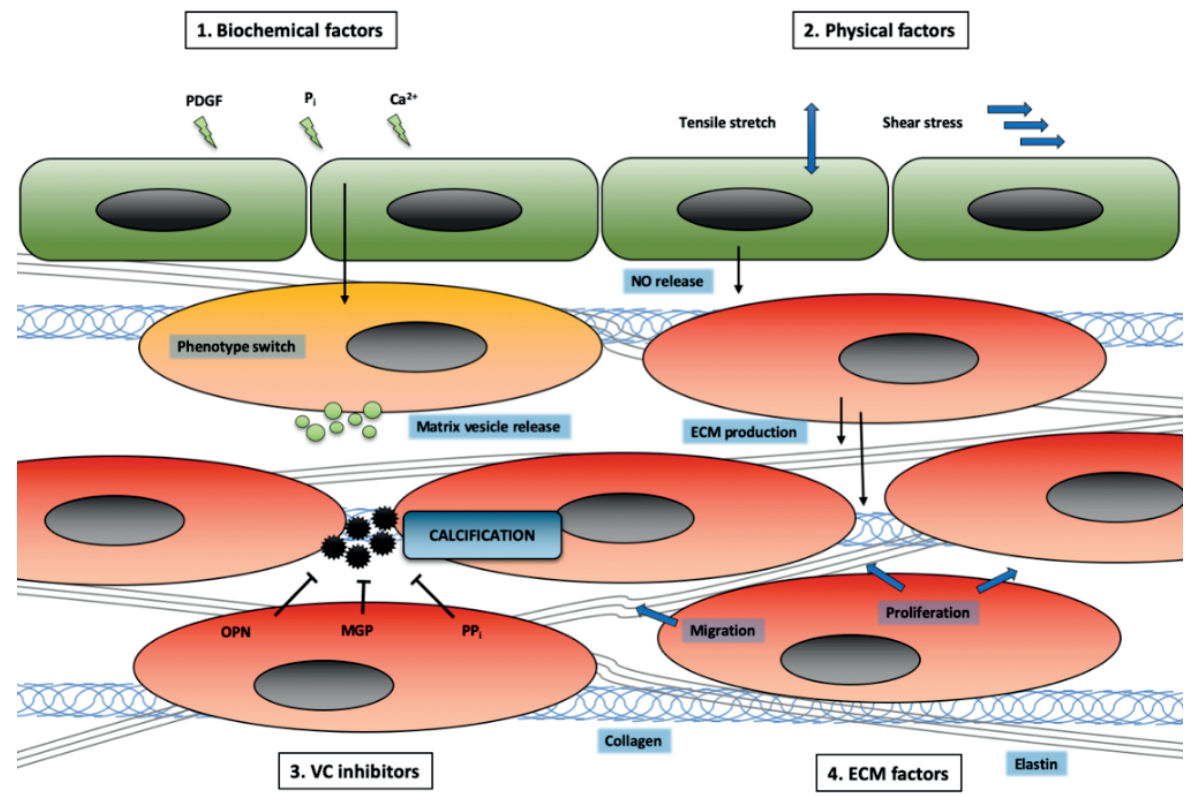

b

Figure 1. (a) Vascular smooth muscle cell (VMSC) phenotypic switching. Under physiological conditions, VSMCs display a contractile phenotype that regulates vessel structure and function. Under stress, arterial remodeling will occur, leading to VSMC phenotypic switching. Factors inducing the synthetic phenotype include platelet derived growth factors (PDGF), protease-activated receptors (PARs) and tumor necrosis factoralpha (TNF- $\alpha$ ). Synthetic VSMCs initiate vessel repair and can switch back to the contractile phenotype, driven by factors such as heparin or laminin. The osteogenic phenotype can be induced by prolonged exposure to BMP-2 or high phosphate. Osteogenic VSMCs shed extracellular vesicles that promote vascular calcification. Panel (b) Pathways affecting vascular calcification. Vascular calcification is an active process which can be initiated by several pathways, including: 1 . biochemical factors, 2. physical factors, 3. vascular calcification (VC) inhibitors and 4. ECM factors. Biochemical factors, such as raised calcium and phosphate levels and PDGF, are stress molecules that induce VSMCs to switch towards a synthetic or osteogenic phenotype, including an increased release of extracellular vesicles. The ECM of the vessel wall directly influences VSMCs. Changes in collagen and elastin content cause VSMCs to change morphology and phenotype. VSMCs in turn, produce MMPs that induce structural changes in the vessel wall by rearranging collagen and elastin, promoting the migration and proliferation of VSMCs and other cell types. Physical factors such as shear stress and tensile stress affect ECs' NO release, which influences the VSMC phenotype. Shear-induced stress induces a synthetic phenotype by decreasing a-SMA, MYH and smtn expression. Tensile stretch induces VSMCs to produce ECM proteins, such as collagen and fibronectin promoting vessel fibrosis. VC inhibitors, such as OPN, MGP and $\mathrm{PP}_{1}$, affect the VSMC phenotype by inducing changes in RNA expression patterns. Taken together, all stimuli play a part in an orchestrated VSMC response, ultimately promoting vascular calcification. 


\section{Biology of Vascular Remodeling}

\subsection{Arterial Remodeling}

Arterial remodeling reflects the adaptation of the vessel wall to biochemical and biomechanical stimuli 9,12. In the vasculature, two types of remodeling can be distinguished: outward and inward remodeling, with respective hypertrophy (thickening) or hypotrophy (thinning) of the vessel wall. Large conduit vessels do not have the ability to constrict in response to stress, and therefore show hypertrophic remodeling. Atherosclerosis is characterized by an increase in vessel diameter, with the thickening of both the media and intima, and classically termed outward hypertrophic remodeling ${ }^{28}$. Aneurysm formation is characterized by an increase in vessel diameter, with a thinning of the vessel wall, and termed outward hypotrophic remodeling ${ }^{29}$ (Figure 2). Inward remodeling is less frequently seen and is observed in more muscular peripheral arteries, probably reflecting the sustained vasoconstriction of vessels ${ }^{30}$.

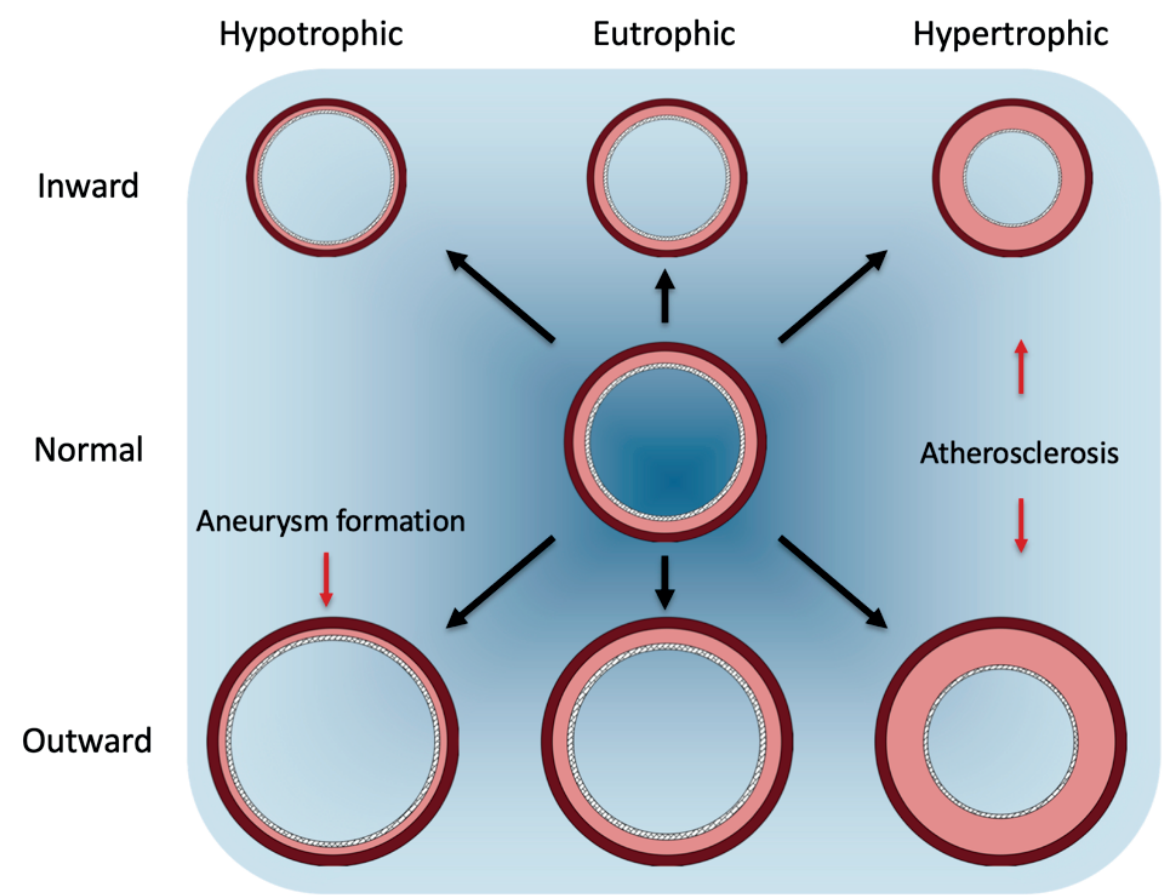

Figure 2. Vascular remodeling types. There are several types of arterial remodeling: hypotrophic, eutrophic and hypertrophic. Additionally, remodeling can be inward and outward. Hypotrophic remodeling results in a thinner vessel wall, which can be both inward and outward. In both cases, the wall-to-lumen ratio decreases. Hypertrophic remodeling results in the thickening of the vessel wall, that can also be inward and outward. The thickening of the vessel wall results in an increased wall-to-lumen ratio. In the eutrophic situation, wall-tolumen ratios do not differ, but the size of the vessel can change. Atherosclerosis is characterized by an increased wall-to-lumen ratio, with both thickening of the media and intima and, therefore, is classified as inward or outward hypertrophic remodeling. Aneurysm formation is characterized by an increase in vessel diameter with a thinning of the vessel wall (outward hypotrophic remodeling). Inward remodeling is less frequent and is more associated with muscular peripheral arteries, reflecting sustained vasoconstriction of vessels. 
Under low laminar flow, platelet derived growth factors (PDGF) and transforming growth factor- $\beta$ (TGF- $\beta$ ) promote inward remodeling by increasing VSMC proliferation and collagen deposition ${ }^{31}$. Inward remodeling also contributes to atherosclerosis, but does not lead to an increased vessel size (Figure 2). Atherosclerosis development involves many cellular processes, like the recruitment of inflammatory cells by chemotaxis and infiltration ${ }^{32}$.

VSMCs are connected to a fenestrated network of elastin and collagen fibers. The capacity of the vessel wall to elastically distend is important to accommodate the volume ejected with each heartbeat and to limit peripheral pressure pulsations. The active tone and spatial arrangement of VSMCs may influence the mechanical load on the ECM components and, therefore, modulate vessel diameter and stiffness ${ }^{18}$. Chronic exposure to high blood pressure increases tensile stress ${ }^{9}$ to which VSMCs respond by proliferation, resulting in hyperplasia and thickening of the vascular wall. Concomitant activity of MMPs facilitates the structural breakdown of elastin ECM, and synthetic VSMCs produce collagen ECM, attempting to preserve stiffness homeostasis ${ }^{33}$. High blood pressure thus aggravates age-related stiffening of arteries ${ }^{34-36}$. Additional ECM disturbances, such as the presence of calcium crystals, have a further impact on the stiffening of the medial layer ${ }^{37,38}$.

During aging, it is generally accepted that the number of cells in the vasculature decreases, although the causes of this finding remain to be established ${ }^{34}$. It has been hypothesized that VSMCs become senescent and that cell division rates decrease ${ }^{39}$. The recent literature ignores vessel wall cellularity and often refers to cellular processes, such as apoptosis, inflammation, calcification and epigenetic effects, all playing a part in vessel wall aging ${ }^{20}$. Additionally, with aging, collagen content in major arterial vasculature increases, whereas elastin content decreases and the number of VSMCs declines ${ }^{40}$. As a consequence, remaining VSMCs are embedded in a collagen-enriched ECM with fewer cellular focal adhesions ${ }^{41-43}$. Within arterial vessels, differences exist in the content of elastin to VSMC ratios ${ }^{44}$. Large arteries close to the heart contain more elastin and are therefore called "elastic" arteries. It is particularly elastic arteries that stiffen with age ${ }^{45}$. Large artery stiffening results in decreased arterial compliance, especially in those aged over 60 years ${ }^{19,46,47}$. Peripheral vessels contain more VSMCs relative to elastin and are termed "muscular" arteries. In muscular arteries, the relative elastin content increases with age, most likely caused by the decline in the number of VSMCs and decreased collagen content ${ }^{44}$. It should be noted that the absolute amount of ECM proteins in the vasculature decreases with age, but that fat and extracellular material, such as calcium crystals, increase ${ }^{48}$. Taken together, the number of VSMCs within the vasculature strongly correlates with vascular stiffening and the arterial remodeling processes ${ }^{34}$.

Endothelial function plays an important role in arterial remodeling. Blood flow and normal wall shear stress stimulate ECs to produce NO (Figure 3). NO induces the relaxation of local VSMCs, which leads to dilation of the vessel wall. Endotheliumdependent vasodilation decreases with age, which appears to be associated (perhaps causally or bi-directionally) with a quiescent state of VSMCs and is clearly implicated to be associated with hypertension and CVD ${ }^{49,50}$. Under pathological conditions, ECs are known to produce cytokines and growth factors ${ }^{51}$, which induces VSMCs phenotype switching from contractile to a more synthetic phenotype. An increased wall shear stress increases endothelial derived NO release, further decreasing VSMC proliferation and 
increasing VSMC apoptosis ${ }^{52}$, resulting in outward remodeling and stiffened vasculature, due to the formation of the ECM matrix by remaining synthetic VSMCs 34,53 .

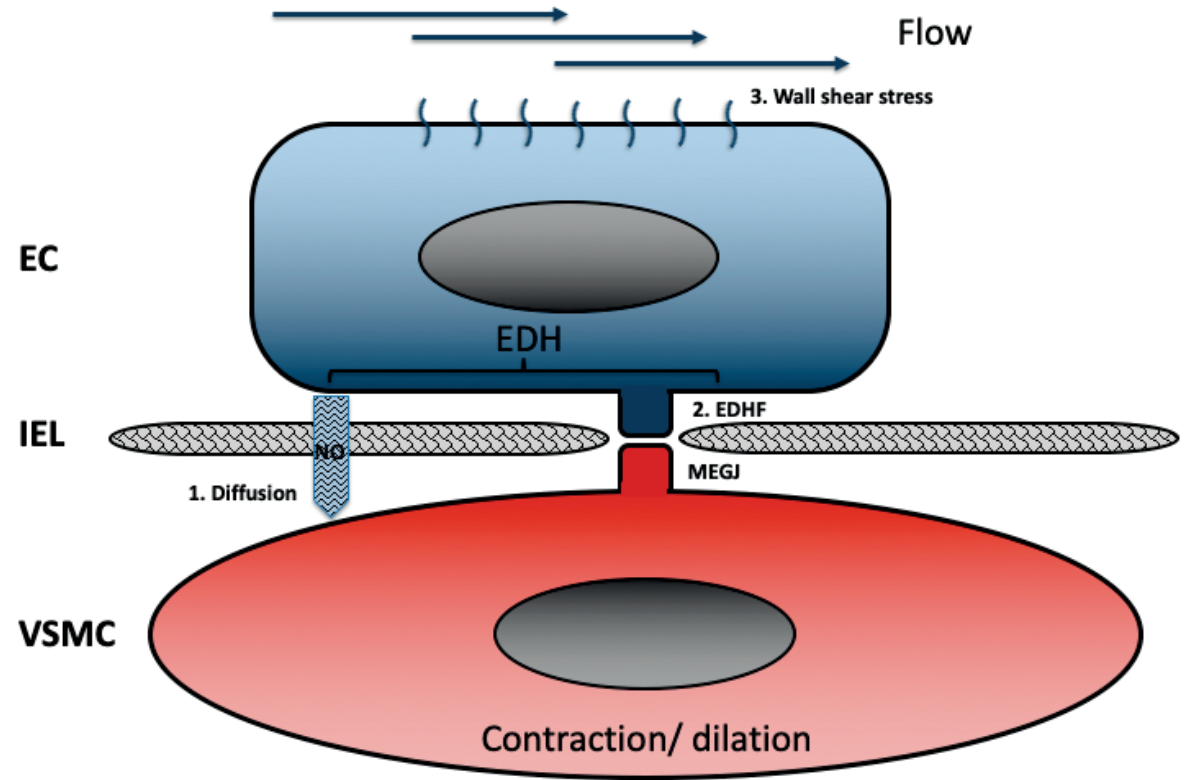

Figure 3. Endothelial cell-smooth muscle cell communication. Endothelial cells (ECs) communicate with vascular smooth muscle cells (VSMCs) in two ways, called endothelial derived hyperpolarization (EDH). EDH has two major pathways: 1. Diffusion of nitric oxide (NO), which is produced by ECs and diffuses through the internal elastic lamina (IEL) to induce the relaxation of VSMCs. 2. Signaling through myoendothelial gap junctions (MEGJ) that connect ECs directly to VSMCs and cross the IEL. This direct communication between ECs and VSMC via MEGJ occurs via so-called endothelial derived hyperpolarizing factors (EDHF). 3. Major factors that stimulate EC NO secretion are flow or shear-stress alterations. Differences in wall shear rates are known to induce endothelial-derived changes in VSMC phenotype.

\subsubsection{Inflammation and Arterial Remodeling}

Inflammatory processes play a significant role in arterial remodeling and are linked to the development of atherosclerosis. Chronic low-grade inflammation is a key driver of arterial aging. VSMC phenotypic switching and ECM biochemical changes activate inflammatory pathways and oxidative stress signaling ${ }^{54}$. Cytokines determine processes such as endothelial dysfunction, renin angiotensin activation and metalloproteinases release ${ }^{55,56}$. VSMCs are involved in local vessel wall inflammatory functions.

\section{Proliferation}

VSMCs have low turnover rates in healthy vessels, but increase proliferation upon vascular injury to initiate repair ${ }^{57}$. Increased expression of aging genes p16 $6^{\mathrm{INK} 4 \mathrm{a}}$ and p21 ${ }^{58}$ have been linked to VSMC senescence and are present in atherosclerotic plaques, indicating lower proliferation and, thus, decreased repair capacity ${ }^{59}$. 


\section{Cell Death}

Cell death is linked to vascular disease, but absolute rates are difficult to determine. VSMC apoptosis in atherosclerosis has been linked to plaque vulnerability features ${ }^{60}$, and chronic VSMC apoptosis has been shown to promote atherogenesis and plaque progression ${ }^{61}$. In vascular aging and medial remodeling, VSMCs cell death does not cause significant inflammation, due to the efficient IL-1 $\beta$-mediated clearance of apoptotic bodies by VSMCs ${ }^{62}$.

\section{Platelets and Extracellular Vesicles (EVs)}

Platelets have shown to affect VSMC inflammatory phenotypes and injury responses ${ }^{63}$. Additionally, platelet EVs induce an inflammatory response in VSMC in vitro ${ }^{64}$. Platelet factor 4 (PF4) has a central role in the stimulation of VSMC-mediated cytokine release, which is primarily affected by increased krüppel-like factor 4 (KLF-4) transcription. Experimental atherosclerosis models indicated that PF4 is proatherogenic and it has been found to penetrate deep into the vessel wall, underlining the importance of platelets aside from their effect on the endothelium ${ }^{65}$.

\subsection{The Role of VSMC Plasticity in Vascular Remodeling}

Under physiological conditions, VSMCs display a contractile phenotype, in which markers such as alpha-smooth muscle actin ( $\alpha$-SMA), smooth muscle 22-alpha (SM22- $\alpha$ ), smoothelin A/B (smtn), smooth muscle-myosin heavy chain (MYC11) and Calponin-1 (CNN-1) are highly expressed ${ }^{14,66,67}$ (Table 1). VSMCs are heterogeneous with regard to their expression, are derived from different embryonic origins and have a strong genetic component causing VSMC diversity. Numerous in vivo studies have shown the existence of diverse VSMC populations within the same artery in rats ${ }^{68}$, pigs ${ }^{69}$ and humans ${ }^{70}$. We refer to VSMC plasticity here as the capability of VSMCs to switch phenotype. VSMC phenotypes are changed by environmental cues, such as soluble biochemical compounds, ECM proteins and biophysical conditions ${ }^{33,71}$ (Figure 1). VSMCs exhibit a whole array of phenotypes, ranging from contractile-quiescent to migratory-proliferative-synthetic and osteogenic-, macrophage- or MSC-like ${ }^{72-74}$ (Table 1). In the following subsections, we will briefly describe the most relevant factors that affect VSMC phenotype (Figure 1 and Table 2).

Table 1. Proteins that define VSMC phenotype.

\begin{tabular}{|c|c|c|c|c|}
\hline \multirow{2}{*}{ Protein } & \multirow{2}{*}{ Gene Name } & \multirow{2}{*}{ Abbreviation } & \multicolumn{2}{|c|}{ Expression } \\
\hline & & & Contractile & Synthetic \\
\hline Alpha smooth muscle actin & $A C T A-2$ & $\alpha-\mathrm{SMA}$ & + & + \\
\hline Smooth muscle myosin heavy chain & MYH11 & SMMHC & + & - \\
\hline Smooth muscle 22 alpha & $T A G L N$ & $\mathrm{SM} 22-\alpha$ & + & - \\
\hline Smoothelin & $S M T N$ & Smtn & + & - \\
\hline Calponin & $C N N-1$ & CNN-1 & + & - \\
\hline Tumor necrosis factor alpha & $T N F A$ & TNF- $\alpha$ & & + \\
\hline S100 calcium binding protein A4 & S100A4 & $\mathrm{S} 100 \mathrm{~A} 4$ & - & + \\
\hline Monocyte chemoattractant protein 1 & $C C L 2$ & MCP-1 & - & + \\
\hline
\end{tabular}

+ , positive effect; -, negative effect. 
Table 2. Factors involved in VSMC phenotype switching.

\begin{tabular}{ccc}
\hline Factors Involved in VSMC Phenotype Switching & \multicolumn{2}{c}{ Phenotype } \\
\hline Biochemical compounds & Contractile & Synthetic \\
\hline PDGF & - & + \\
TGF- $\beta$ & + & - \\
PARs & - & + \\
TNF- $\alpha$ & + & + \\
Angiotensin II & + & + \\
Extracellular matrix components & + & - \\
Integrin: $\alpha 1 \beta 1, \alpha 7 \beta 1, \alpha 8 \beta 1$ & - & + \\
Integrin: $\alpha 2 \beta 1, \alpha 5 \beta 1, \alpha v \beta 3$ & - & + \\
Collagen type I & + & - \\
Collagen type IV & + & - \\
Elastin & + & + \\
Heparin & - & - \\
Fibronectin & + & + \\
Laminin & & + \\
Physical factors & + & + \\
Tensile stress & & + \\
Shear stress & - & + \\
Transcription & & + \\
KLF4 & & + \\
Oct4 & & + \\
\hline
\end{tabular}

Abbreviations: PDGF, platelet derived growth factor; TGF- $\beta$, transforming growth factor beta; PARs Proteaseactivated receptors; TNF- $\alpha$, tumor necrosis factor alpha; KLF4, krüppel-like factor 4; Oct4, octamer-binding transcription factor $4 .+$, positive effect; -, negative effect.

\subsubsection{Biochemical Compounds}

PDGF is an important signaling molecule in the initial phase of VSMC differentiation. During vascular development, PDGF causes mesenchymal cell recruitment and subsequent proliferation ${ }^{75}$. PDGF is known to induce VSMC differentiation towards a synthetic phenotype, as it downregulates the contractile marker aSMA in aortic VSMCs ${ }^{76}$. Additionally, PDGF increases proliferation and migration in pig and human coronary VSMCs ${ }^{69,77,78}$. Moreover, in vivo studies showed that inhibition of PDGF results in reduced VSMC proliferation and migration after arterial balloon injury ${ }^{79,80}$.

TGF- $\beta$, as opposed to PDGF, induces a contractile and non-proliferative VSMC phenotype. An absence of TGF- $\beta$ results in severe congenital cardiovascular disease, mainly shown in structural defects in experimental animals ${ }^{81}$. In vitro treatment of VSMCs with TGF- $\beta$ induces expression of $\alpha$ SMA, MHC and CNN1 ${ }^{69,82,83}$.

Activated coagulation proteins also have been shown to affect VSMC phenotype via protease activated receptors (PARs) ${ }^{84}$. Both tumor necrosis factor-alpha $(\mathrm{TNF}-\alpha)$ and angiotensin II have been shown to induce the contractile, as well as the synthetic, phenotype of VSMCs. It has been shown that angiotensin II affects VSMC phenotypic plasticity via the induction of ROS and decreased scavenging activity of nitrate reductase $(\mathrm{NAD}(\mathrm{P}) \mathrm{H})$ oxidases ${ }^{85}$ and subsequently induces aneurysm formation via oxidative stress ${ }^{21,86}$. 


\subsubsection{Extracellular Components}

The ECM in which VSMCs are embedded also affects VSMC phenotype. VSMC phenotype is modulated via integrin receptors that are present on ECM proteins ${ }^{87}$. ECM consists of structural proteins, such as collagen, elastin and proteoglycans. For instance, the proteoglycan heparin promotes VSMC contractility. In vitro, heparin treatment of VSMCs induces a contractile phenotype and slows down its proliferation ${ }^{69,88}$. Collagen has pleiotropic effects on VSMC phenotype, depending on the type of collagen. Collagen type-I and fibronectin induce a synthetic VSMC phenotype ${ }^{89,90}$ with proliferation ${ }^{91,92}$. On the contrary, collagen type-IV and laminin promote a contractile VSMC phenotype ${ }^{89,93}$. Intact elastin is associated with a contractile VSMC phenotype. The loss of elastin, by a genetic mutation, is associated with increased hypertrophy and hyperplasia of VSMCs ${ }^{94,95}$. Additionally, elastin remodeling, by the modulation of cathepsins and MMPs, promotes VSMC phenotypic switching that induces calcification 96,97

Not only composition, but also the organization of structural fibers, determines VSMC phenotype. Culturing VSMCs in $3 \mathrm{D}$ compared to $2 \mathrm{D}$ increases contractile expression markers and induces TGF- $\beta$ expression ${ }^{98,99}$. Culturing VSMCs on scaffold templates controls spatial organization and morphological response of VSMCs, indicating that VSMCs react to structural environmental changes to retain vessel function 100 .

\subsubsection{Biophysical Factors}

Hemodynamics at the vessel level (flow and transmural pressure) also affect VSMCs by determining tensile loading and active tone (e.g., by NO). Changes in vessel wall shear stress modulate EC NO release, and influence VSMCs via cell-cell interaction with ECs ${ }^{101}$. Using co-cultures of ECs and VSMCs, shear stress induces a synthetic VSMC phenotype by decreasing $\alpha$-SMA, MYH and smtn expression ${ }^{69}$. However, 2D co-culture systems do not mimic the complex vessel wall architecture in vivo. Changes in blood pressure directly transfer into changes in ECM tensile stress, which, as determined by (local) matrix elastic behavior and VSMC stiffness, results in VSMC deformation (i.e., mechanical strain). Alterations in VSMC strain are understood to modulate cellular phenotype ${ }^{33}$. Mechanical stretching forces applied directly to VSMCs enhance the expression of ECM proteins, such as collagen and fibronectin 102 . Biophysical factors in the physiological range promote VSMCs to maintain or adopt a contractile phenotype, whereas pathological biophysical stimuli promotes VSMCs to switch towards a synthetic phenotype.

\subsubsection{Transcriptional Regulators}

KLF4 is an important transcriptional regulator defining VSMC phenotype during development ${ }^{103}$ and after vascular injury ${ }^{104}$. KLF4 expression is increased in lesions of $\mathrm{ApoE}^{-/-}$mice on a Western type diet ${ }^{105}$. Additionally, there is increased binding of KLF4 to $\alpha$ SMA and SM22 $\alpha$ promotors upon vascular injury in mouse carotid arteries ${ }^{104}$. The loss of KLF4 has favorable effects, inhibiting plaque pathogenesis and reducing plaque vulnerability ${ }^{106}$. KLF4 is critical in the regulation of phenotypic switching of VSMCs, both in vitro and in vascular injury models. KLF4 expression results in profound activation of pluripotency genes such as Oct4 and Sox2, indicating that VSMCs can reactivate its pluripotency network in response to vascular stress or 
damage ${ }^{5}$. Moreover, KLF4 activates $>800$ pro-inflammatory VSMC genes, of which many are atherosclerosis relevant ${ }^{106}$. Additionally, KLF4 knock-out in an atherosclerosis animal model revealed the switch of VSMCs toward a synthetic phenotype, while suppressing the macrophage-like form ${ }^{106}$.

\section{Clinical Features of Vascular Remodeling}

\subsection{Hypertension}

Hypertension is widely accepted as a risk factor for the development of CVD. However, hypertension was first considered to be a consequence of aging and seen as insurmountable ${ }^{107}$. Later, several studies revealed that hypertension was associated with increased cardiovascular mortality ${ }^{108,109}$. A meta-analysis on blood pressure and cardiovascular disease showed that a rise of $20 \mathrm{mmHg}$ in systolic blood pressure (SBP) and of $10 \mathrm{mmHg}$ in diastolic blood pressure (DBP) was associated with a more than twofold increase in vascular mortality ${ }^{110}$. More recently, the CALIBER study revealed that patients with hypertension (defined as $>140 / 90 \mathrm{~mm} \mathrm{Hg}$ or those receiving blood pressure-lowering drugs) have a lifetime risk for overall CVD of $63.3 \%$ at 30 years of age (compared to $46.1 \%$ in patients with normal blood pressure) and develop CVD 5 years earlier ${ }^{111}$. Decreasing the SBP by $20 \mathrm{mmHg}$ was associated with a $39 \%$ reduction in cardiovascular (CV) events in the total group and in a $69 \%$ reduction in patients between 60 and 69 years of age ${ }^{112}$. Currently, much of the focus on the primary and secondary prevention of CVD revolves around control of blood pressure ${ }^{112}$.

The systolic aspect of hypertension is quite strongly determined by age- and disease-related decreases in arterial compliance ${ }^{19,113-115}$. Therefore, arterial remodeling processes affecting medial elastic properties are directly relevant in risk profiling and as treatment targets.

\subsubsection{Cellular Components and Hypertension}

High blood pressure is a multifactorial disorder in which genetic alterations, environmental factors and comorbidities interact ${ }^{26,116}$. ECs play an important role in the development of hypertension. In health, ECs are exposed to physiological shear stress, which is necessary to maintain proper functioning of the endothelium. The NO excreted by ECs regulates vascular tone, and thereby preserves ECM and VSMCs' functioning ${ }^{117}$. In pathology, blood flow is more oscillatory, with higher peaks and blood stasis during diastole ${ }^{118}$. Turbulent flow and changes in shear stress, either high or low, affect EC function. Low shear stress areas are considered prone to developing atherosclerosis, inducing pro-inflammatory pathways and lead to dysregulation of the cytoskeleton and junctional proteins of ECs ${ }^{117,119,120}$. High shear stress induces morphological changes in ECs, as they align in the direction of the flow. Additionally, arterial outward remodeling occurs, leading to increased NO synthesis ${ }^{121,122}$.

In hypertension, the amplitude and rate of elastic distension of arteries are increased, causing fatigue, damage and degradation of the vessel wall ECM ${ }^{34}$. Moreover, excessive VSMC strains induce phenotypic switching of these VSMCs to more synthetic types and, consequently, promotes arterial fibrosis, (further) compromising arterial compliance $^{123}$. 


\subsubsection{Calcification and Hypertension}

Several pathologies are associated with VSMC phenotype switching ${ }^{14}$. Synthetic VSMCs produce EVs ${ }^{2,124,125}$, that have been found in both the intimal and medial layers of the vessel wall ${ }^{126}$. VSMCs are known to release EVs upon phenotypic switching towards synthetic or osteogenic phenotype. EVs derived from VSMCs share similarities with EVs from osteoblasts, having calcium-binding capacities and osteoblast-like ECM production ${ }^{2,83}$. Recently, it was shown that not all EVs promote calcification ${ }^{127}$. This implies that EV content is variable and dependent on VSMCmediated biogenesis. A specific subclass of EVs are exosomes, which express tetraspanins (CD9, CD63 and CD81) and differ in expression pattern, and, hence, mineralizing capacity ${ }^{127}$. Furthermore, calcifying conditions in vitro increase the multivesicular body, forming enzyme sphingomyelin phosphodiesterase 3 (SMPD3) and subsequent exosome genesis ${ }^{83}$. Consequently, the inhibition of SMPD3 ablates the generation of exosomes and their subsequent calcification. Additionally, sortilin has been suggested as a key player in the VSMC-mediated calcifying EVs genesis and release. Sortilin is a sorting receptor that directs target proteins to a designated location via the secretory or endocytic compartment ${ }^{128}$. Recent findings have identified that sortilin promotes vascular calcification via the trafficking and loading of tissuenonspecific alkaline phosphatase into EVs ${ }^{129}$. Moreover, sortilin co-localized with calcification in human calcified vessels ${ }^{129}$. Differences between mineralizing and nonmineralizing EVs eventually determine calcification of the ECM in proximity of VSMCs. Hence, better insight into EV composition, such as lipid content and RNA and protein profile, might provide new insights into the mechanism by which VSMC derived EVs contribute to vascular calcification and result in novel targets for treatment.

In response to calcified ECM, neighboring ECs and VSMCs react and induce the production of osteogenic factors, such as bone morphogenetic protein 2 (BMP-2) and 4 (BMP-4) ${ }^{130,131}$. Besides this expression of bone-associated proteins, the number of VSMCs decreases with age, and an increase of collagen-to-elastin ratio further increases the stiffening of the vessel wall ${ }^{132}$.

In hypertension, calcium handling is disturbed, which is associated with an increased activation of L-type calcium channels and sensitivity of (hypertensive) patients toward calcium channel blockers ${ }^{133,134}$. Increased intracellular calcium induces the activation of receptors coupled to phospholipase $\mathrm{C}$, leading to the generation of second messengers that trigger cytokines, ROS and miRNAs, and also cellular derived EVs ${ }^{135}$. Additionally, increased intracellular calcium activates the contractile machinery of VSMCs, leading to hyper-contractility. Excessive intracellular calcium rapidly disintegrates both mitochondria and structural components of VSMCs, and results in calcium depositions within elastic fibers ${ }^{136}$. Consequently, elasticity of the vasculature decreases, further contributing to increased blood pressure ${ }^{137,138}$ and VSMC stiffening ${ }^{135}$. Specific calcium antagonists, blocking L-type voltage-dependent calcium channels, reduce calcium internalization and normalize blood pressure ${ }^{26,133}$. Of note, these calcium channel blockers also prevent calcification of the vasculature ${ }^{139,140}$.

\subsubsection{Consequences of Vascular Calcification}

Vascular calcification is associated with arterial stiffness ${ }^{141-143}$. In chronic kidney disease, medial calcification of large arteries is directly responsible for the increased stiffness (or reduced distensibility) ${ }^{144}$. The presence of vascular calcification 
(process) in the vessel wall contributes to ECM degradation and wall thickening ${ }^{34,145}$. Taken together, vascular calcification contributes directly and indirectly to arterial stiffening and, ultimately, hypertension ${ }^{146,147}$. Atherosclerosis has also been associated with arterial stiffness and hypertension ${ }^{51,148}$. Additionally, increased blood pressure and wall shear stress increase vulnerability and, consequently, atherosclerotic plaques become prone to rupture ${ }^{149,150}$.

\subsection{Atherosclerosis}

Atherosclerosis is characterized by intimal plaque build-up and often referred to as a thickening of the vessel wall, ultimately narrowing the lumen of the vessel. Atherosclerosis is considered an inflammatory disease, starting with endothelial stress and subsequent monocyte infiltration ${ }^{151,152}$. Clinically, plaques may rupture, resulting in stroke or myocardial infarction ${ }^{32,153}$. Besides the classical view that systemic factors are causative for atherosclerosis, recent data suggest that local vascular processes also initiate atherogenesis ${ }^{154,155}$. Lineage tracing studies revealed that many of the cells present in atherosclerotic plaques are derived from VSMC precursors ${ }^{106}$. Additionally, it is now recognized that atherosclerotic plaque macrophages have lineage traces of VSMCs, indicating that cells in the intima originate from the vascular media, and not necessarily from the circulating mesenchymal cells that infiltrate the vessel wall at sites of injury ${ }^{106}$.

Arterial remodeling is considered key to the development of atherosclerosis ${ }^{32}$. Arterial remodeling of the vessel wall can be initiated via many processes, including oxidative stress ${ }^{156}$, proliferation ${ }^{152,157}$, VSMC phenotype switching ${ }^{2}$, cell infiltration, apoptosis and calcification ${ }^{60}$.

\subsection{Intimal and Medial Aspects of Vascular Calcification}

Vascular calcification can be found at two anatomical locations, the medial and intimal layer. In the 1940s, post-mortem analysis of aortic specimens from CVD patients revealed that atherosclerosis is only present at vascular areas with either medial degradation or medial calcification ${ }^{158}$. Histological examination of aortas showed that medial calcification preceded atherosclerotic plaque build-up. Intimal calcification is seen as a hallmark of atherosclerosis and, clinically, is used as a marker for atherosclerotic burden ${ }^{159-161}$. For a long time, calcification has been considered as a passive degenerative detrimental process, not amendable for intervention. This view has recently changed, since statin treatment is associated with both increased plaque stability and increased plaque calcification ${ }^{162-165}$. However, the apparent protective role of calcification in stabilizing plaque is in contrast with the predictive value of calcification for CVD morbidity and mortality ${ }^{166}$. Moreover, medial calcification has been shown to change blood dynamics by inducing arterial remodeling processes and increasing arterial stiffness ${ }^{167-169}$. Medial calcification strongly correlates with the phenotypical switching of VSMCs and a rise in blood pressure. Macrocalcifications in patients who underwent a computed tomography (CT) scan were shown to have a three- to four-fold risk of developing fatal cardiovascular events ${ }^{164,168}$. Additionally, microcalcifications, which are precursors of macrocalcification, have been shown to induce plaque vulnerability ${ }^{170-}$ 172 . 


\section{Cellular Processes of Vascular Calcification}

Specific stimuli, such as elevated calcium or phosphate levels, induce the switching of VSMCs to an osteogenic phenotypic where the cells acquire features of chondrocytes and osteoblasts ${ }^{173}$. Osteogenic VSMCs show an increased expression of osteogenic markers, such as alkaline phosphatase, BMP-2 and runt-related transcription factor 2 (Runx2), but show decreased calcification-inhibitor protein expression ${ }^{174}$. VSMCs exposed to elevated calcium levels display an intracellular calcium overload that may induce microcalcifications, eventually resulting in macrocalcification, which contributes to vascular stiffness and hypertension ${ }^{26}$. The process of calcification, culminating in either micro- or macrocalcification, compromises the structural integrity of the vessel wall and, hence, its functional properties.

Oxidative stress is an important player in the development of vascular calcification. Oxidative stress pathways in VSMCs have been linked to development aortic valve calcification ${ }^{175}$. Increased oxidative stress induces expression of Runx2, a key transcription factor associated with osteoblast differentiation ${ }^{176}$. VSMC-specific Runx2 knockout was shown to decrease vascular calcification and atherosclerosis development ${ }^{177}$. Additionally, increased $\mathrm{H}_{2} \mathrm{O}_{2}$ levels have been found in the close vicinity of calcification nucleation foci, together with an enhanced expression of oxidases ${ }^{178}$. High phosphate levels, present in chronic kidney disease, are associated with extent of vascular calcification ${ }^{179}$. In vitro, phosphate has been shown to induce mitochondrial-derived free radicals, by enhancing mitochondrial membrane potential ${ }^{180}$. Antioxidants inhibit phosphate-induced ROS production ${ }^{180}$. Increased oxidative stress is also found in diabetic patients ${ }^{181}$, leading to an increased accumulation of advanced glycation end products (AGEs). AGEs have been shown to induce vascular calcification via increased oxidative stress of VSMC ${ }^{182,183}$.

\subsection{Fibrosis}

Fibrosis of different organs contributes to up to $50 \%$ of worldwide mortality ${ }^{184}$. In the vasculature, myofibroblasts produce ECM proteins such as collagen and fibronectin ${ }^{185}$. Myofibroblasts are derived from MSC-like cells that reside in the vessel wall. The role of these MSC-like cells in the vessel wall is not fully understood, and is believed to play an important role in structural vessel repair. In vascular fibrosis, excessive collagen deposition leads to a reduced compliance of the vessel, which indicates increased arterial stiffness. Furthermore, high blood pressure induces structural changes including hypertrophy of the vessel wall ${ }^{186,187}$ and cellular ${ }^{14}$ and ECM remodeling ${ }^{188,189}$.

Lineage tracing studies have shown that resident $\mathrm{Glil}^{+}$MSC-like cells are a major contributor of vascular fibrosis in arterial remodeling ${ }^{190}$. Acute vessel wall injury revealed that a significant number of $\mathrm{Gli1}^{+}$cells were detected in the media and neointima, and that they express VSMC markers such as $\alpha \mathrm{SMA}$ and CNN1. Additionally, some $50 \%$ of the newly formed VSMCs were derived from $\mathrm{Gli}^{+}$ progenitors, indicating the involvement of adventitial cells in arterial remodeling ${ }^{191}$. Likewise, angiotensin II induced hypertension was caused by an exuberant production of collagen in the adventitial layer ${ }^{192}$. VSMCs that migrate towards the adventitia start expressing stem cell markers Sca-1 and CD34 ${ }^{193}$. These "adventitial stem cells" change towards fibroblasts and deposit collagen and other ECM, contributing to adventitial fibrosis ${ }^{194}$. 
During atherosclerosis development, fate-tracing studies in mice have shown that MSC-like cells migrate into the media and neo-intima. Also, MSC-like Gli1 ${ }^{+}$cells were a major source of osteoblasts-like cells, significantly contributing to the process of vascular calcification in both vascular media and intima. The genetic ablation of $\mathrm{Gli}^{+}$ cells before injury dramatically reduced the severity of vascular calcification ${ }^{191}$. These data indicate that fibrotic processes are regulated by myofibroblasts and MSC-like cells and that these are important in the development of atherosclerosis and vascular calcification.

\section{Assessing Vascular Remodeling and Disease}

\subsection{Pulse Wave Velocity (PWV), Distensibility Coefficient (DC) and Intima-Media Thickness (IMT) \\ Carotid-femoral PWV is considered the gold standard for assessing central} arterial stiffness ${ }^{195,196}$. PWV, however, only measures vascular characteristics of the aorta and does not harbor information on any other vessels. Multi-slice CT, measuring calcification, reflects the atherosclerotic burden, and IMT measures atherosclerosis in the carotid arteries. Below, we describe in more detail measures of vascular remodeling and disease.

\subsubsection{Pulse Wave Velocity}

Measuring arterial stiffness in the clinic is challenging. The methods that are available for measuring arterial stiffness are non-invasive vascular imaging, such as ultrasound and magnetic resonance imaging (MRI), and high-fidelity recording of the pulse wave, performed by tonometry or ultrasound Doppler. Functional measures that can be obtained are pulse wave velocity (PWV) and the distensibility coefficient (DC). In the clinic, carotid-femoral PWV (cfPWV) adds to existing risk scores in cardiovascular risk management ${ }^{197}$, while arterial stiffening has been shown to be independently associated with cardiovascular risk and mortality ${ }^{198,199}$. Brachial-ankle PWV is also used as an alternative to cfPWV, correlating reasonably well ${ }^{200}$.

The interpretation of arterial stiffness findings in human studies is subject to (1) the stiffness measure/method used and (2) the mechanistic or constitutive focus of the study. Constitutive properties that are often considered to interpret arterial stiffness findings are those related to the $\mathrm{ECM}$ or $\mathrm{VSMCs}^{18}$.

\subsubsection{Distensibility Coefficient}

Arterial stiffness can be determined by measuring local vessel diameter and distension by ultrasound or MRI ${ }^{18}$. The measured data can be used to calculate the local distensibility coefficient (DC) or compliance. PWV, as well as DC, are well known to be pressure dependent and require blood pressure adjustment ${ }^{201,202}$. Adjustment is valid when considering group analyses, but lacks applicability in the use of individual cases 203 .

PWV is largely dependent on the elastic properties of the large arteries. Therefore, PWV should be interpreted with care, since it only reflects the stiffness of the aorta. In conduit arteries, the relative amount of VSMCs are limited and, thus, the contribution of ECM to arterial stiffening may be expected to be more pronounced. In peripheral, more muscular, arteries, age-related stiffening is not as straightforward ${ }^{204}$. 
PWV in the lower extremities can change significantly, which may explain why no evident relation between PWV and age has been reported 34,205. Additionally, hypertrophy of VSMCs is commonly observed in hypertension ${ }^{206,207}$, with the potential to (partially) determine PWV ${ }^{208}$. Thus, PWV is an important clinical marker for risk assessment, as it correlates with cardiovascular risk, mortality, and organ damage of the heart, kidney and brains ${ }^{209}$.

Techniques for measuring PWV are developing. cfPWV is most commonly used for aortic stiffness, but is susceptible to local wave reflections and not suitable for obtaining information on carotid arteries ${ }^{210}$. Ultrasound ultrafast imaging techniques are being explored to directly measure the propagation of the pulse wave, with limited success for the common carotid artery ${ }^{211,212}$. Elastography is a promising technique, as it measures the propagation of shear waves in the tissue ${ }^{213}$. The propagation of the shear wave is directly proportional to the elastic modulus of the tissue ${ }^{214}$. PWV can also be measured using MRI, which also allows for segmentation. Its drawbacks are costs and long scanning times ${ }^{215}$. Currently, the available PWV toolbox can only be applied to large and medium-sized arteries, but techniques are developing for smaller vessels.

At present, the interpretation of stiffness measurements in terms of arterial wall constitutive properties remains a challenge ${ }^{18}$. Medial calcifications are known to colocalize with elastin fragmentation spots, but the mechanical contribution of the calcific deposits to vessel stiffness remains to be established; the elastin degradation alone could be sufficient to explain stiffening ${ }^{35}$.

\subsubsection{Intima-Media Thickness}

IMT is used as a measurement for assessing the thickness of the tunica intima and media (estimated from lumen-intima and media-adventitia echo complexes). IMT is often measured using ultrasound and can be readily obtained in superficial arteries. One aspect that is not measured by IMT is adventitial thickness is a significant contributor of pathology development ${ }^{192}$. Wall thickness data are important in determining arterial wall material stiffness and wall stress. But, IMT can also be used to detect atherosclerosis, and is used to follow progression of the disease ${ }^{216}$. IMT is shown to be an independent predictor of overall cardiovascular events, including myocardial infarction and stroke ${ }^{217}$. Multiple studies have shown that increases in IMT can be significantly delayed using effective cardiovascular drugs, such as statins ${ }^{218,219}$. However, further observational studies revealed that this delay did not result in reduced cardiovascular events ${ }^{220}$.

To study large populations, non-invasive techniques such as IMT are preferred over invasive measurement tools. As such, IMT provides a reliable tool for assessing vessel wall macro-structural changes. However, some limitations for IMTs exist. There is a lack of standardized protocols for the use of IMT, and attempts to establish them have not reached consensus yet ${ }^{221,222}$. IMTs of certain segments, such as the common carotid artery, are related to hypertension, blood pressure and vascular hypertrophy ${ }^{223}$, whereas the internal carotid artery correlates with atherosclerotic plaques ${ }^{224}$. Taken together, additional risk assessments, such as plaque presence and stenosis severity, will be complementary to the use of IMT alone. A novel technique to determine extra-media thickness may have great potential in combination with IMT to discriminate between the medial and adventitial layer which aids in risk assessment ${ }^{225}$. Moreover, other imaging techniques, such as coronary artery calcium (CAC) scanning, have been demonstrated to be superior for predicting CVD events ${ }^{226,227}$. 


\subsection{Calcification Imaging by Computed Tomography}

Calcification imaging in the clinic is mostly based on CT, which is an angiography technique based on X-ray radiation and makes use of computer processing to create a detailed 3D image. Calcification scores, such as the coronary artery calcium (CAC) score, can be derived from CT scans and, in recent decades, this has been used as a measure of atherosclerotic burden. The Agatson score is the most frequently used method to calculate CAC. The Agatston score is defined as a weighed sum of the calcification area, with density given as Hounsfield Units ${ }^{228,229}$. CAC represents an accurate measure to assess atherosclerotic burden ${ }^{159,230}$. Additionally, CAC correlates with traditional risk factors for atherosclerosis, such as hypertension, hypercholesterolemia and diabetes ${ }^{231}$. CAC also associates with cardiovascular morbidities and mortalities, including cardiac death and stroke ${ }^{232,233}$.

$\mathrm{CAC}$ is regarded as the best clinical predictor for the accumulation and progression of vascular calcification over time ${ }^{232,233}$. CAC can be used to identify highrisk individuals that need immediate medical attention or intervention ${ }^{234}$. Interventions to slow down or even regress calcification, using the supplementation of vitamin $\mathrm{K}$, are currently being conducted ${ }^{235-237}$. High dietary intake of vitamin $\mathrm{K}$ is associated with a lower risk of coronary heart disease ${ }^{238}$ and supplementation with vitamin $\mathrm{K}$ significantly delays the progression of calcification ${ }^{239}$. Conversely, statin treatment is known for its beneficial cardiovascular effects, however, it is also shown to increase CAC score ${ }^{240-242}$. This indicates that type of calcification, as well as the volume or density of calcification, determines whether it is plaque-stabilizing or plaque-destabilizing. Indeed, CAC volume is positively and independently associated with CVD, whereas CAC density was inversely correlated with CVD risk ${ }^{243}$.

Measuring CAC as a clinical marker for CVD has some limitations. Firstly, $\mathrm{CAC}$ is limited to coronary arteries, which do not represent the calcification of other vessels of the arterial system. CAC does not represent total plaque size, as histopathological studies showed that the area of calcium deposition was much smaller than total plaque volume ${ }^{244}$. Additionally, CAC score poorly correlated with luminal narrowing and severity ${ }^{245}$. Secondly, distinction between medial and intimal calcification is not possible with current resolutions of CT. Intimal and medial calcification are different pathologies, with different risk factors for developing CVD 246,247 . Additionally, microcalcification $(<15 \mu \mathrm{m})$ cannot be assessed, as the resolution of CT is some $200 \mu \mathrm{m}^{222,248,249}$.

Thirdly, evidence suggests that atherosclerotic plaques that have denser calcification have smaller lipid cores, with increased stability of plaques, resulting in lower CVD risk ${ }^{250}$. Greater CAC volume, however, is associated with a higher risk of $\mathrm{CVD}^{251}$. This indicates that future research should integrate density and volume into CAC scoring. Finally, CAC involves exposure to radiation, which limits the use of calcium scores as a follow-up on atherosclerosis progression ${ }^{252}$.

\section{3. ${ }^{18} \mathrm{~F}-\mathrm{NaF}$ Positron Emission Tomography}

${ }^{18} \mathrm{~F}-\mathrm{NaF}$ PET scanning gained recent attention as a novel imaging modality for vascular calcification ${ }^{253}$. In bone, ${ }^{18} \mathrm{~F}-\mathrm{NaF}$ is incorporated into newly formed hydroxyapatite crystals by exchanging fluoride with the hydroxyl groups ${ }^{254}$. In the vasculature, ${ }^{18} \mathrm{~F}-\mathrm{NaF}$ PET imaging has been reported to identify active 
(micro)calcification with high specificity ${ }^{255}$. Importantly, CT and echocardiography cannot identify active vascular calcification, which is considered the main driver of disease progression ${ }^{256}$. Assessing the molecular bioactivity of calcification in atherosclerotic plaques is of additive value to identify vulnerable plaques ${ }^{256-258}$. Additionally, ex vivo histological studies showed that the resolution of ${ }^{18} \mathrm{~F}-\mathrm{NaF}$ has the potential to discriminate between micro-and macrocalcification ${ }^{257}$. Taken together, ${ }^{18} \mathrm{~F}$ $\mathrm{NaF}$ uptake provides complementary information to $\mathrm{CT}$, as it allows for the detection of ongoing active calcification of plaques, which is a major improvement to the prediction of future cardiovascular risk. Limitations of ${ }^{18} \mathrm{~F}-\mathrm{NaF}$ PET imaging are the relatively high costs and exposure to radiation, but, also, ${ }^{18} \mathrm{~F}-\mathrm{NaF}$ PET imaging lacks the resolution to discriminate between medial and intimal calcification.

\section{Conclusions}

Arterial remodeling is defined as the adaptation of the vessel wall away from a normal homeostatic state towards pathologic development, triggered by chronic exposure to stress signals. Different arterial remodeling processes have been described, such as inflammation, oxidative stress, lipid accumulation, and degradation of the ECM. Many of these are regulated by VSMCs and affect vessel wall morphology and properties. VSMCs exhibit cellular plasticity and, upon stress stimuli, they may differentiate towards synthetic, macrophage-like and osteogenic VSMCs. As a consequence, VSMCs initiate and support remodeling processes involving ECM synthesis and cell proliferation, migration and contraction, which aid in maintaining tissue functionality. Disruption of VSMC phenotypic plasticity has many pathological consequences, including the development of hypertension, atherosclerosis, aneurysm formation, intimal and medial calcification, and vascular fibrosis, ultimately leading to increased CVD morbidity and mortality. Early detection of vascular remodeling is key in the prevention and prognosis of CVD. Clinically, assessment of calcification-mediated remodeling remains difficult. Assessment tools include measurements of pulse wave velocity, distensibility coefficient and intima-media thickness. These techniques may capture changes in dynamic and geometric vessel wall properties, but cannot directly monitor the underlying arterial remodeling processes. The addition of techniques, such as ${ }^{18} \mathrm{~F}-\mathrm{NaF}$ PET and $\mathrm{CT}$ imaging may provide further mechanistic insight into vascular calcification development and other VSMC-mediated processes.

Combining expertise in vascular biology, clinical vascular expertise and multiscale/multi-modality imaging should advance the understanding and identification of critical determinants of vascular remodeling, ultimately resulting in tailor-made diagnoses and treatments. 


\section{References}

1. Mazurek, R. et al. Vascular Cells in Blood Vessel Wall Development and Disease. Advances in Pharmacology 78, 323-350 (2017).

2. Schurgers, L. J. et al. Initiation and Propagation of Vascular Calcification Is Regulated by a Concert of Platelet- and Smooth Muscle Cell-Derived Extracellular Vesicles. Front Cardiovasc Med 5, 36 (2018).

3. Roostalu, U. \& Wong, J. K. Arterial smooth muscle dynamics in development and repair. Dev. Biol. 435, 109-121 (2018).

4. Hao, H., Gabbiani, G. \& Bochaton-Piallat, M.-L. Arterial smooth muscle cell heterogeneity: implications for atherosclerosis and restenosis development. Arteriosclerosis, Thrombosis, and Vascular Biology 23, 1510-1520 (2003).

5. Alexander, M. R. \& Owens, G. K. Epigenetic control of smooth muscle cell differentiation and phenotypic switching in vascular development and disease. Annu. Rev. Physiol. 74, 13-40 (2012).

6. van Bussel, F. C. G. et al. A control systems approach to quantify wall shear stress normalization by flow-mediated dilation in the brachial artery. PLOS ONE 10, e0115977 (2015).

7. van der Heijden, O. W. H. et al. Uterine artery remodeling and reproductive performance are impaired in endothelial nitric oxide synthase-deficient mice. Biol. Reprod. 72, 1161-1168 (2005).

8. Boutouyrie, P. et al. Common carotid artery stiffness and patterns of left ventricular hypertrophy in hypertensive patients. Hypertension 25, 651-659 (1995).

9. O'Rourke, M. F. \& Nichols, W. W. Aortic Diameter, Aortic Stiffness, and Wave Reflection Increase With Age and Isolated Systolic Hypertension. Hypertension 45, 652-658 (2005).

10. Libby, P. Inflammation in atherosclerosis. Nature 420, 868-874 (2002).

11. Henning, R. J., Bourgeois, M. \& Harbison, R. D. Poly(ADP-ribose) Polymerase (PARP) and PARP Inhibitors: Mechanisms of Action and Role in Cardiovascular Disorders. Cardiovasc. Toxicol. 18, 493-506 (2018).

12. van Varik, B. J. et al. Mechanisms of arterial remodeling: lessons from genetic diseases. Front Genet 3, 290 (2012).

13. Lacolley, P., Regnault, V., Segers, P. \& Laurent, S. Vascular Smooth Muscle Cells and Arterial Stiffening: Relevance in Development, Aging, and Disease. Physiological Reviews 97, 1555-1617 (2017).

14. Owens, G. K., Kumar, M. S. \& Wamhoff, B. R. Molecular Regulation of Vascular Smooth Muscle Cell Differentiation in Development and Disease. Physiological Reviews 84, 767-801 (2004).

15. Willis, A. I., Pierre-Paul, D., Sumpio, B. E. \& Gahtan, V. Vascular smooth muscle cell migration: current research and clinical implications. Vasc Endovascular Surg 38, 11-23 (2004).

16. Johnson, J. L. Matrix metalloproteinases: influence on smooth muscle cells and atherosclerotic plaque stability. Expert Rev Cardiovasc Ther 5, 265-282 (2014).

17. Peeters, S. A. et al. Plasma levels of matrix metalloproteinase-2, -3, -10, and tissue inhibitor of metalloproteinase- 1 are associated with vascular complications in patients with type 1 diabetes: the EURODIAB Prospective Complications Study. Cardiovasc Diabetol 14, 31 (2015).

18. Reesink, K. D. \& Spronck, B. Constitutive interpretation of arterial stiffness in clinical studies: a methodological review. Am. J. Physiol. Heart Circ. Physiol. 316, H693-H709 (2019).

19. Smulyan, H., Mookherjee, S. \& Safar, M. E. The two faces of hypertension: role of aortic stiffness. J Am Soc Hypertens 10, 175-183 (2016).

20. Lacolley, P., Regnault, V., Nicoletti, A., Li, Z. \& Michel, J.-B. The vascular smooth muscle cell in arterial pathology: a cell that can take on multiple roles. Cardiovasc. Res. 95, 194-204 (2012).

21. Petsophonsakul, P. et al. Role of Vascular Smooth Muscle Cell Phenotypic Switching and Calcification in Aortic Aneurysm Formation. Arteriosclerosis, Thrombosis, and Vascular Biology 27, ATVBAHA119312787 (2019).

22. Pawade, T. A., Newby, D. E. \& Dweck, M. R. Calcification in Aortic Stenosis: The Skeleton Key. J. Am. Coll. Cardiol. 66, 561-577 (2015).

23. Neven, E. \& D'Haese, P. C. Vascular calcification in chronic renal failure: what have we learned from animal studies? Circ. Res. 108, 249-264 (2011).

24. Proudfoot, D. Molecular mechanisms of arterial calcification. Artery Research 3, 128-131 (2009).

25. Tyson, K. L. et al. Osteo/chondrocytic transcription factors and their target genes exhibit distinct patterns of expression in human arterial calcification. Arteriosclerosis, Thrombosis, and Vascular Biology 23, 489-494 (2003).

26. Fleckenstein-Grün, G., Frey, M., Thimm, F., Hofgärtner, W. \& Fleckenstein, A. Calcium Overload - An Important Cellular Mechanism in Hypertension and Arteriosclerosis. Drugs 44, 23-30 (1992). 
Shanahan, C. M. Mechanisms of vascular calcification in CKD-evidence for premature ageing? Nat Rev Nephrol 9, 661-670 (2013).

O'Rourke, M. F. \& Hashimoto, J. Mechanical Factors in Arterial Aging: A Clinical Perspective. J. Am. Coll. Cardiol. 50, 1-13 (2007).

Mulvany, M. J. et al. Vascular remodeling. Hypertension 28, 505-506 (1996).

Mulvany, M. J. Small artery remodelling in hypertension: causes, consequences and therapeutic implications. Med Biol Eng Comput 46, 461-467 (2008).

Ward, M. R., Pasterkamp, G., Yeung, A. C. \& Borst, C. Arterial remodeling. Mechanisms and clinical implications. Circulation 102, 1186-1191 (2000).

Hansson, G. K. \& Libby, P. The immune response in atherosclerosis: a double-edged sword. Nature Reviews Immunology 2006 6:7 6, 508-519 (2006).

Humphrey, J. D., Dufresne, E. R. \& Schwartz, M. A. Mechanotransduction and extracellular matrix homeostasis. Nat. Rev. Mol. Cell Biol. 15, 802-812 (2014).

Greenwald, S. E. Ageing of the conduit arteries. J. Pathol. 211, 157-172 (2007).

Spronck, B. et al. A constitutive modeling interpretation of the relationship among carotid artery stiffness, blood pressure, and age in hypertensive subjects. Am. J. Physiol. Heart Circ. Physiol. 308, H568-82 (2015).

Humphrey, J. D., Harrison, D. G., Figueroa, C. A., Lacolley, P. \& Laurent, S. Central Artery Stiffness in Hypertension and Aging: A Problem With Cause and Consequence. Circ. Res. 118, 379-381 (2016).

Sekikawa, A. et al. Aortic stiffness and calcification in men in a population-based international study. Atherosclerosis 222, 473-477 (2012).

Kruger, T. et al. Warfarin Induces Cardiovascular Damage in Mice. Arteriosclerosis, Thrombosis, and Vascular Biology 33, 2618-2624 (2013).

Chi, C. et al. Vascular smooth muscle cell senescence and age-related diseases: State of the art. Biochim Biophys Acta Mol Basis Dis 1865, 1810-1821 (2019).

Sans, M. \& Moragas, A. Mathematical morphologic analysis of the aortic medial structure. Biomechanical implications. Anal. Quant. Cytol. Histol. 15, 93-100 (1993).

Cliff, W. The aortic tunica media in aging rats. Experimental and Molecular Pathology 13, 172 189 (1970).

Zieman, S. J. \& Kass, D. A. Advanced Glycation Endproduct Crosslinking in the Cardiovascular System. Drugs 64, 459-470 (2004).

Wang, J. C. \& Bennett, M. Aging and Atherosclerosis. Circ. Res. 111, 245-259 (2012).

Leondes, C. T. Biomechanical Systems : Techniques and Applications, Volume II: Cardiovascular Techniques. Biomechanical Systems (CRC Press, 2000). doi:10.1201/9781420049534

Kelly, R., Hayward, C., Avolio, A. \& O'Rourke, M. Noninvasive determination of age-related changes in the human arterial pulse. Circulation 80, 1652-1659 (1989).

Laogun, A. A. \& Gosling, R. G. In vivo arterial compliance in man. Clin Phys Physiol Meas 3, 201-212 (1982).

Zieman, S. J., Melenovsky, V. \& Kass, D. A. Mechanisms, Pathophysiology, and Therapy of Arterial Stiffness. Arteriosclerosis, Thrombosis, and Vascular Biology 25, 932-943 (2005).

Cattell, M. A., Anderson, J. C. \& Hasleton, P. S. Age-related changes in amounts and concentrations of collagen and elastin in normotensive human thoracic aorta. Clinica Chimica Acta 245, 73-84 (1996).

Sun, Z. Aging, arterial stiffness, and hypertension. Hypertension 65, 252-256 (2015).

Brandes, R. P., Fleming, I. \& Busse, R. Endothelial aging. Cardiovasc. Res. 66, 286-294 (2005). Urschel, K., Cicha, I., Daniel, W. G. \& Garlichs, C. D. Shear stress patterns affect the secreted chemokine profile in endothelial cells. Clin. Hemorheol. Microcirc. 50, 143-152 (2012).

Qiu, J. et al. Biomechanical regulation of vascular smooth muscle cell functions: from in vitro to in vivo understanding. $J$ R Soc Interface 11, 20130852 (2014).

3. Yu, H., Clarke, M. C. H., Figg, N., Littlewood, T. D. \& Bennett, M. R. Smooth muscle cell apoptosis promotes vessel remodeling and repair via activation of cell migration, proliferation, and collagen synthesis. Arteriosclerosis, Thrombosis, and Vascular Biology 31, 2402-2409 (2011).

Mozos, I. \& Luca, C. T. Crosstalk between Oxidative and Nitrosative Stress and Arterial Stiffness. Curr Vasc Pharmacol 15, 446-456 (2017).

McMaster, W. G., Kirabo, A., Madhur, M. S. \& Harrison, D. G. Inflammation, immunity, and hypertensive end-organ damage. Circ. Res. 116, 1022-1033 (2015). 


\section{Chapter 2}

56.

Jain, S., Khera, R., Corrales-Medina, V. F., Townsend, R. R. \& Chirinos, J. A. "Inflammation and arterial stiffness in humans". Atherosclerosis 237, 381-390 (2014).

Lutgens, E. et al. Biphasic pattern of cell turnover characterizes the progression from fatty streaks to ruptured human atherosclerotic plaques. Cardiovasc. Res. 41, 473-479 (1999).

Matthews, C. et al. Vascular smooth muscle cells undergo telomere-based senescence in human atherosclerosis: effects of telomerase and oxidative stress. Circ. Res. 99, 156-164 (2006).

Moon, S.-K., Cha, B.-Y. \& Kim, C.-H. In vitro cellular aging is associated with enhanced proliferative capacity, G1 cell cycle modulation, and matrix metalloproteinase-9 regulation in mouse aortic smooth muscle cells. Arch. Biochem. Biophys. 418, 39-48 (2003).

Clarke, M. C. H. et al. Apoptosis of vascular smooth muscle cells induces features of plaque vulnerability in atherosclerosis. Nat. Med. 12, 1075-1080 (2006).

Clarke, M. C. H. et al. Chronic apoptosis of vascular smooth muscle cells accelerates atherosclerosis and promotes calcification and medial degeneration. Circ. Res. 102, 1529-1538 (2008).

Bennett, M. R., Gibson, D. F., Schwartz, S. M. \& Tait, J. F. Binding and phagocytosis of apoptotic vascular smooth muscle cells is mediated in part by exposure of phosphatidylserine. Circ. Res. 77, 1136-1142 (1995).

Shi, G. \& Morrell, C. N. Platelets as initiators and mediators of inflammation at the vessel wall. Thromb. Res. 127, 387-390 (2011).

Vajen, T. et al. Platelet extracellular vesicles induce a pro-inflammatory smooth muscle cell phenotype. J Extracell Vesicles 6, 1322454 (2017).

Shi, G. et al. Platelet factor 4 mediates vascular smooth muscle cell injury responses. Blood 121,

$4417-4427$ (2013).
Miano, J. M. Serum Mol. Cell. Cardiol. 35, 577-593 (2003).

. Rensen, S. S. M., Doevendans, P. A. F. of vascular smooth muscle cell phenotypic diversity. Neth Heart J 15, 100-108 (2007).

Bochaton-Piallat, M.-L., Ropraz, P., Gabbiani, F. \& Gabbiani, G. Phenotypic Heterogeneity of Rat Arterial Smooth Muscle Cell Clones. Arteriosclerosis, Thrombosis, and Vascular Biology 16, 815-820 (1996).

Hao, H. et al. Heterogeneity of Smooth Muscle Cell Populations Cultured From Pig Coronary Artery. Arteriosclerosis, Thrombosis, and Vascular Biology 22, 1093-1099 (2002).

$\mathrm{Li}$, S. et al. Innate diversity of adult human arterial smooth muscle cells: cloning of distinct subtypes from the internal thoracic artery. Circ. Res. 89, 517-525 (2001).

Wanjare, M., Kuo, F. \& Gerecht, S. Derivation and maturation of synthetic and contractile vascular smooth muscle cells from human pluripotent stem cells. Cardiovasc. Res. 97, 321-330 (2013).

Gerthoffer, W. T. Mechanisms of vascular smooth muscle cell migration. Circ. Res. 100, 607-621 (2007).

Frismantiene, A., Philippova, M., Erne, P. \& Resink, T. J. Smooth muscle cell-driven vascular diseases and molecular mechanisms of VSMC plasticity. Cellular Signalling 52, 48-64 (2018).

Allahverdian, S., Chaabane, C., Boukais, K., Francis, G. A. \& Bochaton-Piallat, M.-L. Smooth muscle cell fate and plasticity in atherosclerosis. Cardiovasc. Res. 114, 540-550 (2018).

Schattemann, G. C., Loushin, C., Li, T. \& Hart, C. E. PDGF-A is required for normal murine cardiovascular development. Dev. Biol. 176, 133-142 (1996).

Li, X. et al. Suppression of smooth-muscle $\alpha$-actin expression by platelet-derived growth factor in vascular smooth-muscle cells involves Ras and cytosolic phospholipase A2. Biochemical Journal 327, 709-716 (1997).

Li, J.-Y. et al. Inhibitory effect of PDGF-BB and serum-stimulated responses in vascular smooth muscle cell proliferation by hinokitiol via up-regulation of p21 and p53. Arch Med Sci 14, 579587 (2018).

Ishigami, M., Swertfeger, D. K., Granholm, N. A. \& Hui, D. Y. Apolipoprotein E inhibits plateletderived growth factor-induced vascular smooth muscle cell migration and proliferation by suppressing signal transduction and preventing cell entry to G1 phase. J. Biol. Chem. 273, 2015620161 (1998).

Kotani, M. et al. Chimeric DNA-RNA hammerhead ribozyme targeting PDGF A-chain mRNA specifically inhibits neointima formation in rat carotid artery after balloon injury. Cardiovasc. Res. 57, 265-276 (2003). 
80. Deguchi, J. et al. Targeting endogenous platelet-derived growth factor B-chain by adenovirusmediated gene transfer potently inhibits in vivo smooth muscle proliferation after arterial injury. Gene Therapy 1999 6:6 6, 956-965 (1999).

81. Sanford, L. P. et al. TGFbeta2 knockout mice have multiple developmental defects that are nonoverlapping with other TGFbeta knockout phenotypes. Development 124, 2659-2670 (1997).

82. Hautmann, M. B., Madsen, C. S. \& Owens, G. K. A transforming growth factor beta (TGFbeta) control element drives TGFbeta-induced stimulation of smooth muscle alpha-actin gene expression in concert with two CArG elements. J. Biol. Chem. 272, 10948-10956 (1997).

83. Kapustin, A. N. et al. Vascular smooth muscle cell calcification is mediated by regulated exosome secretion. Circ. Res. 116, 1312-1323 (2015).

84. Borissoff, J. I., Spronk, H. M. H. \& Cate, ten, H. The hemostatic system as a modulator of atherosclerosis. N. Engl. J. Med. 364, 1746-1760 (2011).

85. Li, F.-F., Shang, X.-K., Du, X.-L. \& Chen, S. Rapamycin Treatment Attenuates Angiotensin II induced Abdominal Aortic Aneurysm Formation via VSMC Phenotypic Modulation and Downregulation of ERK1/2 Activity. Curr Med Sci 38, 93-100 (2018).

86. Jiang, F. et al. Angiotensin-converting enzyme 2 and angiotensin 1-7: novel therapeutic targets. Nature Reviews Cardiology 2014 11:7 11, 413-426 (2014).

87. Moiseeva, E. P. Adhesion receptors of vascular smooth muscle cells and their functions. Cardiovasc. Res. 52, 372-386 (2001).

88. Zhang, J. et al. ECM-mimetic heparin glycosamioglycan-functionalized surface favors constructing functional vascular smooth muscle tissue in vitro. Colloids Surf B Biointerfaces 146, 280-288 (2016).

89. Sazonova, O. V. et al. Extracellular matrix presentation modulates vascular smooth muscle cell mechanotransduction. Matrix Biol. 41, 36-43 (2015).

90. Finney, A. C., Stokes, K. Y., Pattillo, C. B. \& Orr, A. W. Integrin signaling in atherosclerosis. Cell. Mol. Life Sci. 74, 2263-2282 (2017).

91. Ichii, T. et al. Fibrillar collagen specifically regulates human vascular smooth muscle cell genes involved in cellular responses and the pericellular matrix environment. Circ. Res. 88, 460-467 (2001).

92. Chung, C. H., Lin, K. T., Chang, C. H., Peng, H. C. \& Huang, T. F. The integrin $\alpha 2 \beta 1$ agonist, aggretin, promotes proliferation and migration of VSMC through NF-kB translocation and PDGF production. British Journal of Pharmacology 156, 846-856 (2009).

93. Thyberg, J. \& Hultgårdh-Nilsson, A. Fibronectin and the basement membrane components laminin and collagen type IV influence the phenotypic properties of subcultured rat aortic smooth muscle cells differently. Cell Tissue Res 276, 263-271 (1994).

94. O'Connor, W. N. et al. Supravalvular aortic stenosis. Clinical and pathologic observations in six patients. Arch. Pathol. Lab. Med. 109, 179-185 (1985).

95. Bunton, T. E. et al. Phenotypic alteration of vascular smooth muscle cells precedes elastolysis in a mouse model of Marfan syndrome. Circ. Res. 88, 37-43 (2001).

96. Bouvet, C., Moreau, S., Blanchette, J., de Blois, D. \& Moreau, P. Sequential activation of matrix metalloproteinase 9 and transforming growth factor beta in arterial elastocalcinosis. Arteriosclerosis, Thrombosis, and Vascular Biology 28, 856-862 (2008).

97. Pai, A., Leaf, E. M., El-Abbadi, M. \& Giachelli, C. M. Elastin degradation and vascular smooth muscle cell phenotype change precede cell loss and arterial medial calcification in a uremic mouse model of chronic kidney disease. The American Journal of Pathology 178, 764-773 (2011).

98. Li, S. et al. Genomic analysis of smooth muscle cells in 3-dimensional collagen matrix1. The FASEB Journal 17, 97-99 (2002).

99. Tijore, A., Behr, J.-M., Irvine, S. A., Baisane, V. \& Venkatraman, S. Bioprinted gelatin hydrogel platform promotes smooth muscle cell contractile phenotype maintenance. Biomed Microdevices 20, 32 (2018).

100. Sarkar, S., Dadhania, M., Rourke, P., Desai, T. A. \& Wong, J. Y. Vascular tissue engineering: microtextured scaffold templates to control organization of vascular smooth muscle cells and extracellular matrix. Acta Biomater 1, 93-100 (2005).

101. van Engeland, N. C. A. et al. A biomimetic microfluidic model to study signalling between endothelial and vascular smooth muscle cells under hemodynamic conditions. Lab Chip 18, 16071620 (2018).

102. O'Callaghan, C. J. \& Williams, B. Mechanical Strain-Induced Extracellular Matrix Production by Human Vascular Smooth Muscle Cells. Hypertension 36, 319-324 (2000). 


\section{Chapter 2}

103. Cordes, K. R. et al. miR-145 and miR-143 regulate smooth muscle cell fate and plasticity. Nature 11, 441 (2009).

104. Yoshida, T., Kaestner, K. H. \& Owens, G. K. Conditional Deletion of Krüppel-Like Factor 4 Delays Downregulation of Smooth Muscle Cell Differentiation Markers but Accelerates Neointimal Formation Following Vascular Injury. Circ. Res. 102, 1548-1557 (2008).

105. Cherepanova, O. A. et al. Oxidized Phospholipids Induce Type VIII Collagen Expression and Vascular Smooth Muscle Cell Migration. Circ. Res. 104, 609-618 (2009).

106. Shankman, L. S. et al. KLF4-dependent phenotypic modulation of smooth muscle cells has a key role in atherosclerotic plaque pathogenesis. Nat. Med. 21, 628-637 (2015).

107. Kotchen, T. A. Historical trends and milestones in hypertension research: a model of the process of translational research. Hypertension 58, 522-538 (2011).

108. Franklin, S. S. \& Wong, N. D. Hypertension and cardiovascular disease: contributions of the framingham heart study. Glob Heart 8, 49-57 (2013).

109. Dawber, T. R. et al. Some factors associated with the development of coronary heart disease: six years' follow-up experience in the Framingham study. Am J Public Health Nations Health 49, 1349-1356 (1959).

110. Lewington, S. et al. Age-specific relevance of usual blood pressure to vascular mortality: a metaanalysis of individual data for one million adults in 61 prospective studies. The Lancet $\mathbf{3 6 0}, 1903-$ 1913 (2002).

111. Rapsomaniki, E. et al. Blood pressure and incidence of twelve cardiovascular diseases: lifetime risks, healthy life-years lost, and age-specific associations in 1.25 million people. Lancet $\mathbf{3 8 3}$, 1899-1911 (2014).

112. Rahimi, K., Emdin, C. A. \& MacMahon, S. The epidemiology of blood pressure and its worldwide management. Circ. Res. 116, 925-936 (2015).

113. Najjar, S. S. et al. Pulse wave velocity is an independent predictor of the longitudinal increase in systolic blood pressure and of incident hypertension in the Baltimore Longitudinal Study of Aging. J. Am. Coll. Cardiol. 51, 1377-1383 (2008).

114. Franklin, S. S. \& Levy, D. Aging, blood pressure, and heart failure: what are the connections? Hypertension 58, 760-762 (2011).

115. Verwoert, G. C. et al. Arterial stiffness and hypertension in a large population of untreated individuals: the Rotterdam Study. J. Hypertens. 32, 1606-12; discussion 1612 (2014).

116. Harvey, A., Montezano, A. C. \& Touyz, R. M. Vascular biology of ageing-Implications in hypertension. J. Mol. Cell. Cardiol. 83, 112-121 (2015).

117. Gimbrone, M. A. \& García-Cardeña, G. Vascular endothelium, hemodynamics, and the pathobiology of atherosclerosis. Cardiovasc. Pathol. 22, 9-15 (2013).

118. Mitchell, G. F. Increased Aortic Stiffness: An Unfavorable Cardiorenal Connection. Hypertension 43, 151-153 (2004).

119. Dai, G. et al. Distinct endothelial phenotypes evoked by arterial waveforms derived from atherosclerosis-susceptible and -resistant regions of human vasculature. Proc. Natl. Acad. Sci. U.S.A. 101, 14871-14876 (2004).

120. Passerini, A. G. et al. Coexisting proinflammatory and antioxidative endothelial transcription profiles in a disturbed flow region of the adult porcine aorta. Proc. Natl. Acad. Sci. U.S.A. 101, 2482-2487 (2004).

121. Blackman, B. R., García-Cardeña, G. \& Gimbrone, M. A. A New In Vitro Model to Evaluate Differential Responses of Endothelial Cells to Simulated Arterial Shear Stress Waveforms. $J$ Biomech Eng 124, 397-407 (2002).

122. Wang, Y.-X. et al. A multi-component parallel-plate flow chamber system for studying the effect of exercise-induced wall shear stress on endothelial cells. Biomed Eng Online 15, 154 (2016).

123. Williams, B. Mechanical influences on vascular smooth muscle cell function. J. Hypertens. 16, 1921-1929 (1998).

124. Kapustin, A. N. et al. Prothrombin Loading of Vascular Smooth Muscle Cell-Derived Exosomes Regulates Coagulation and Calcification. Arteriosclerosis, Thrombosis, and Vascular Biology 37, e22-e32 (2017).

125. Hutcheson, J. D. et al. Enrichment of calcifying extracellular vesicles using density-based ultracentrifugation protocol. J Extracell Vesicles 3, 25129 (2014).

126. Tanimura, A., McGregor, D. H. \& Anderson, H. C. Matrix Vesicles in Atherosclerotic Calcification 1:. Proceedings of the Society for Experimental Biology and Medicine 172, 173-177 (2016). 
127.

128.

130.

131.

146.

Chen, N. X., O'Neill, K. D. \& Moe, S. M. Matrix vesicles induce calcification of recipient vascular smooth muscle cells through multiple signaling pathways. Kidney Int. 93, 343-354 (2018).

Carlo, A.-S., Nykjaer, A. \& Willnow, T. E. Sorting receptor sortilin-a culprit in cardiovascular and neurological diseases. J. Mol. Med. 92, 905-911 (2014).

Goettsch, C. et al. Sortilin mediates vascular calcification via its recruitment into extracellular vesicles. J. Clin. Invest. 126, 1323-1336 (2016).

Boström, K. I., Rajamannan, N. M. \& Towler, D. A. The regulation of valvular and vascular sclerosis by osteogenic morphogens. Circ. Res. 109, 564-577 (2011).

Shanahan, C. M. et al. Expression of mineralisation-regulating proteins in association with human vascular calcification. $Z$ Kardiol 89 Suppl 2, 63-68 (2000).

Fornieri, C., Quaglino, D. \& Mori, G. Role of the extracellular matrix in age-related modifications of the rat aorta. Ultrastructural, morphometric, and enzymatic evaluations. Arteriosclerosis, Thrombosis, and Vascular Biology 12, 1008-1016 (1992).

Godfraind, T. Discovery and Development of Calcium Channel Blockers. Front Pharmacol 8, 286 (2017).

Zamponi, G. W., Striessnig, J., Koschak, A. \& Dolphin, A. C. The Physiology, Pathology, and Pharmacology of Voltage-Gated Calcium Channels and Their Future Therapeutic Potential. Pharmacol. Rev. 67, 821-870 (2015).

Touyz, R. M. et al. Vascular smooth muscle contraction in hypertension. Cardiovasc. Res. 114, 529-539 (2018).

Georg Thieme Verlag KG et al. A Mechanism Underlying Hypertensive Occurrence in the Metabolic Syndrome: Cooperative Effect of Oxidative Stress and Calcium Accumulation in Vascular Smooth Muscle Cells. Horm Metab Res 46, e2-e2 (2013).

Fleckenstein, A., Fleckenstein-Grün, G., Frey, M. \& Thimm, F. Experimental antiarteriosclerotic effects of calcium antagonists. J Clin Pharmacol 30, 151-154 (1990).

Zhu, Y., He, L., Qu, J. \& Zhou, Y. Regulation of Vascular Smooth Muscle Cell Stiffness and Adhesion by [Ca2+]i: An Atomic Force Microscopy-Based Study. Microsc. Microanal. 24, 708$712(2018)$.

Chen, N. X., Kircelli, F., O'Neill, K. D., Chen, X. \& Moe, S. M. Verapamil inhibits calcification and matrix vesicle activity of bovine vascular smooth muscle cells. Kidney Int. 77, 436-442 (2010).

Caballero-Gonzalez, F. J. Calcium Channel Blockers in the Management of Hypertension in the Elderly. Cardiovascular \& Hematological Agents in Medicinal Chemistry 12, 160-165 (2014).

Niederhoffer, N. et al. Vasodilators, aortic elasticity, and ventricular end-systolic stress in nonanesthetized unrestrained rats. Hypertension 30, 1169-1174 (1997).

London, G. M. et al. Arterial media calcification in end-stage renal disease: impact on all-cause and cardiovascular mortality. Nephrol. Dial. Transplant. 18, 1731-1740 (2003).

Odink, A. E. et al. The association of arterial stiffness and arterial calcification: the Rotterdam study. J Hum Hypertens 22, 205-207 (2008).

Guérin, A. P., London, G. M., Marchais, S. J. \& Metivier, F. Arterial stiffening and vascular calcifications in end-stage renal disease. Nephrol. Dial. Transplant. 15, 1014-1021 (2000).

Wagenseil, J. E. \& Mecham, R. P. Elastin in large artery stiffness and hypertension. J Cardiovasc Transl Res 5, 264-273 (2012).

308, 875-881 (2012).

Sehgel, N. L. et al. Increased vascular smooth muscle cell stiffness: a novel mechanism for aortic stiffness in hypertension. Am. J. Physiol. Heart Circ. Physiol. 305, H1281-H1287 (2013).

Csiszar, A., Lehoux, S. \& Ungvari, Z. Hemodynamic Forces, Vascular Oxidative Stress, and Regulation of BMP-2/4 Expression. https://home.liebertpub.com/ars 11, 1683-1697 (2009).

Richardson, P. D. Biomechanics of plaque rupture: progress, problems, and new frontiers. Ann Biomed Eng 30, 524-536 (2002).

Tang, D. et al. A negative correlation between human carotid atherosclerotic plaque progression and plaque wall stress: in vivo MRI-based 2D/3D FSI models. J Biomech 41, 727-736 (2008).

Hopkins, P. N. Molecular Biology of Atherosclerosis. Physiological Reviews 93, 1317-1542 (2013).

Bennett, M. R., Sinha, S. \& Owens, G. K. Vascular Smooth Muscle Cells in Atherosclerosis. Circ. Res. 118, 692-702 (2016).

Ross, R. Atherosclerosis--an inflammatory disease. N. Engl. J. Med. 340, 115-126 (1999). 
154. Chatrou, M. L. L. et al. Intra-Section Analysis of Human Coronary Arteries Reveals a Potential Role for Micro-Calcifications in Macrophage Recruitment in the Early Stage of Atherosclerosis. PLoS ONE 10, e0142335 (2015).

155. Feil, S. et al. Transdifferentiation of vascular smooth muscle cells to macrophage-like cells during atherogenesis. Circ. Res. 115, 662-667 (2014).

156. Vaidya, D. et al. Association of endothelial and oxidative stress with metabolic syndrome and subclinical atherosclerosis: multi-ethnic study of atherosclerosis. European Journal of Clinical Nutrition 65, 818-825 (2011).

157. Ross, R. \& Glomset, J. A. Atherosclerosis and the arterial smooth muscle cell: Proliferation of smooth muscle is a key event in the genesis of the lesions of atherosclerosis. Science 180, 1332 1339 (1973).

158. Blumenthal, H. Calcification of the media of the human aorta and its relation to intimal arteriosclerosis, ageing and disease. 1-23 (2007).

159. Sangiorgi, G. et al. Arterial Calcification and Not Lumen Stenosis Is Highly Correlated With Atherosclerotic Plaque Burden in Humans: A Histologic Study of 723 Coronary Artery Segments Using Nondecalcifying Methodology. J. Am. Coll. Cardiol. 31, 126-133 (1998).

160. Pundziute, G. et al. Head-to-head comparison of coronary plaque evaluation between multislice computed tomography and intravascular ultrasound radiofrequency data analysis. JACC Cardiovasc Interv 1, 176-182 (2008).

161. Rodriguez-Granillo, G. A., Carrascosa, P., Bruining, N., Waksman, R. \& Garcia-Garcia, H. M. Defining the non-vulnerable and vulnerable patients with computed tomography coronary angiography: evaluation of atherosclerotic plaque burden and composition. Eur Heart J Cardiovasc Imaging 17, 481-491 (2016).

162. Nissen, S. E. Effect of intensive lipid lowering on progression of coronary atherosclerosis: evidence for an early benefit from the Reversal of Atherosclerosis with Aggressive Lipid Lowering (REVERSAL) trial. Am. J. Cardiol. 96, 61F-68F (2005).

163. Ridker, P. M., Pradhan, A., MacFadyen, J. G., Libby, P. \& Glynn, R. J. Cardiovascular benefits and diabetes risks of statin therapy in primary prevention: an analysis from the JUPITER trial. Lancet 380, 565-571 (2012).

164. Puri, R. et al. Long-term effects of maximally intensive statin therapy on changes in coronary atheroma composition: insights from SATURN. Eur Heart J Cardiovasc Imaging 15, 380-388 (2014).

165. Räber, L. et al. Effect of high-intensity statin therapy on atherosclerosis in non-infarct-related coronary arteries (IBIS-4): a serial intravascular ultrasonography study. Eur. Heart J. 36, 490-500 (2015).

166. Rennenberg, R. J. M. W. et al. Vascular calcifications as a marker of increased cardiovascular risk: a meta-analysis. Vasc Health Risk Manag 5, 185-197 (2009).

167. Essalihi, R., Dao, H. H., Yamaguchi, N. \& Moreau, P. A new model of isolated systolic hypertension induced by chronic warfarin and vitamin K1 treatment. Am. J. Hypertens. 16, 103110 (2003).

168. Essalihi, R., Ouellette, V., Hao Dao, H., McKee, M. D. \& Moreau, P. Phenotypic Modulation of Vascular Smooth Muscle Cells During Medial Arterial Calcification: a Role for Endothelin? Journal of Cardiovascular Pharmacology 44, S147-S150 (2004).

169. Lanzer, P. et al. Medial vascular calcification revisited: review and perspectives. Eur. Heart J. 35, 1515-1525 (2014).

170. Ehara, S., Kobayashi, Y. \& Yoshiyama, M. Spotty calcification typifies the culprit plaque in patients with acute myocardial infarction: An intravascular ultrasound study. ACC Current Journal Review 14, 39 (2005).

171. Vengrenyuk, Y. et al. A hypothesis for vulnerable plaque rupture due to stress-induced debonding around cellular microcalcifications in thin fibrous caps. Proc. Natl. Acad. Sci. U.S.A. 103, 1467814683 (2006).

172. Schurgers, L. J. et al. Vitamin K-antagonists accelerate atherosclerotic calcification and induce a vulnerable plaque phenotype. PLOS ONE 7, e43229 (2012).

173. Iyemere, V. P., Proudfoot, D., Weissberg, P. L. \& Shanahan, C. M. Vascular smooth muscle cell phenotypic plasticity and the regulation of vascular calcification. J. Intern. Med. 260, 192-210 (2006).

174. Shanahan, C. M., Crouthamel, M. H., Kapustin, A. \& Giachelli, C. M. Arterial calcification in chronic kidney disease: key roles for calcium and phosphate. Circ. Res. 109, 697-711 (2011). 
175. Liberman, M., Pesaro, A. E. P., Carmo, L. S. \& Serrano, C. V., Jr. Vascular calcification: pathophysiology and clinical implications. Einstein (Sao Paulo) 11, 376-382 (2013).

176. Ducy, P., Zhang, R., Geoffroy, V., Ridall, A. L. \& Karsenty, G. Osf2/Cbfa1: a transcriptional activator of osteoblast differentiation. Cell 89, 747-754 (1997).

177. Sun, Y. et al. Smooth muscle cell-specific runx2 deficiency inhibits vascular calcification. Circ. Res. 111, 543-552 (2012).

178. Liberman, M. et al. Oxidant generation predominates around calcifying foci and enhances progression of aortic valve calcification. Arteriosclerosis, Thrombosis, and Vascular Biology 28, 463-470 (2008).

179. Block, G. A. \& Port, F. K. Re-evaluation of risks associated with hyperphosphatemia and hyperparathyroidism in dialysis patients: recommendations for a change in management. Am. J. Kidney Dis. 35, 1226-1237 (2000).

180. Zhao, M.-M. et al. Mitochondrial reactive oxygen species promote p65 nuclear translocation mediating high-phosphate-induced vascular calcification in vitro and in vivo. Kidney Int. 79, 1071-1079 (2011)

181. Tiwari, B. K., Pandey, K. B., Abidi, A. B. \& Rizvi, S. I. Markers of Oxidative Stress during Diabetes Mellitus. J Biomark 2013, 378790-8 (2013).

182. Wei, Q. et al. Advanced glycation end products accelerate rat vascular calcification through RAGE/oxidative stress. BMC Cardiovasc Disord 13, 13-10 (2013).

183. Tada, Y. et al. Advanced glycation end products-induced vascular calcification is mediated by oxidative stress: functional roles of NAD(P)H-oxidase. Horm Metab Res 45, 267-272 (2013).

184. Friedman, S. L., Sheppard, D., Duffield, J. S. \& Violette, S. Therapy for fibrotic diseases: nearing the starting line. Sci Transl Med 5, 167sr1-167sr1 (2013).

185. Hinz, B. et al. Recent developments in myofibroblast biology: paradigms for connective tissue remodeling. The American Journal of Pathology 180, 1340-1355 (2012).

186. Girerd, X. et al. Noninvasive detection of an increased vascular mass in untreated hypertensive patients. Am. J. Hypertens. 7, 1076-1084 (1994).

187. Fridez, P. et al. Geometrical, functional, and histomorphometric adaptation of rat carotid artery in induced hypertension. J Biomech 36, 671-680 (2003).

188. Safar, M., Chamiot-Clerc, P., Dagher, G. \& Renaud, J. F. Pulse pressure, endothelium function, and arterial stiffness in spontaneously hypertensive rats. Hypertension 38, 1416-1421 (2001).

189. Arribas, S. M., Hinek, A. \& González, M. C. Elastic fibres and vascular structure in hypertension. Pharmacol. Ther. 111, 771-791 (2006).

190. Kramann, R. et al. Perivascular Gli1+ progenitors are key contributors to injury-induced organ fibrosis. Cell Stem Cell 16, 51-66 (2015).

191. Kramann, R. et al. Adventitial MSC-like Cells Are Progenitors of Vascular Smooth Muscle Cells and Drive Vascular Calcification in Chronic Kidney Disease. Cell Stem Cell 19, 628-642 (2016).

192. Bersi, M. R. et al. Excessive Adventitial Remodeling Leads to Early Aortic Maladaptation in Angiotensin-Induced Hypertension. Hypertension 67, 890-896 (2016).

193. Majesky, M. W. et al. Differentiated Smooth Muscle Cells Generate a Subpopulation of Resident Vascular Progenitor Cells in the Adventitia Regulated by Klf4Novelty and Significance. Circ. Res. 120, 296-311 (2017).

194. Wu, J. et al. Origin of Matrix-Producing Cells That Contribute to Aortic Fibrosis in Hypertension. Hypertension 67, 461-468 (2016).

195. Cecelja, M. \& Chowienczyk, P. Role of arterial stiffness in cardiovascular disease. JRSM Cardiovasc Dis 1, (2012).

196. Lee, H.-Y. \& Oh, B.-H. Aging and arterial stiffness. Circ. J. 74, 2257-2262 (2010).

197. Mancia, G. et al. 2013 ESH/ESC Practice Guidelines for the Management of Arterial Hypertension. Blood pressure 23, 3-16 (2014).

198. Nürnberger, J. et al. Augmentation index is associated with cardiovascular risk. J. Hypertens. 20, 2407-2414 (2002).

199. Vlachopoulos, C. V., Terentes-Printzios, D. G., Ioakeimidis, N. K., Aznaouridis, K. A. \& Stefanadis, C. I. Prediction of Cardiovascular Events and All-Cause Mortality With Erectile Dysfunction. Circulation: Cardiovascular Quality and Outcomes 6, 99-109 (2013).

200. Chow, B. \& Rabkin, S. W. Brachial-ankle pulse wave velocity is the only index of arterial stiffness that correlates with a mitral valve indices of diastolic dysfunction, but no index correlates with left atrial size. Cardiol Res Pract 2013, 986847-7 (2013).

201. Spronck, B. et al. Pressure-dependence of arterial stiffness: potential clinical implications. $J$. Hypertens. 33, 330-338 (2015). 
202. Spronck, B. et al. Arterial stiffness index beta and cardio-ankle vascular index inherently depend on blood pressure but can be readily corrected. J. Hypertens. 35, 98-104 (2017).

203. Spronck, B. et al. Patient-specific blood pressure correction technique for arterial stiffness: evaluation in a cohort on anti-angiogenic medication. hypertension research 40, 752-757 (2017).

204. Dao, H. H., Essalihi, R., Bouvet, C. \& Moreau, P. Evolution and modulation of age-related medial elastocalcinosis: impact on large artery stiffness and isolated systolic hypertension. Cardiovasc. Res. 66, 307-317 (2005).

205. Laucyte-Cibulskiene, A. et al. Mismatch between stiffness in elastic and muscular arteries as a predictor of vascular calcification in dialysis patients. Aging Clin Exp Res 30, 375-382 (2018).

206. Sehgel, N. L. et al. Augmented Vascular Smooth Muscle Cell Stiffness and Adhesion When Hypertension Is Superimposed on Aging. Hypertension (2014). doi:10.1161/HYPERTENSIONAHA.114.04456

207. Imanishi, M. et al. Smooth muscle cell-specific Hif-1 $\alpha$ deficiency suppresses angiotensin IIinduced vascular remodelling in mice. Cardiovasc. Res. 102, 460-468 (2014).

208. Gonzalez, W. et al. Molecular plasticity of vascular wall during N(G)-nitro-L-arginine methyl ester-induced hypertension: modulation of proinflammatory signals. Hypertension 36, 103-109 (2000).

209. Laurent, S. \& Boutouyrie, P. Arterial stiffness and stroke in hypertension: therapeutic implications for stroke prevention. CNS Drugs 19, 1-11 (2005).

210. Segers, P., Swillens, A., Taelman, L. \& Vierendeels, J. Wave reflection leads to over- and underestimation of local wave speed by the PU- and QA-loop methods: theoretical basis and solution to the problem. Physiol Meas 35, 847-861 (2014).

211. Hermeling, E., Reesink, K. D., Kornmann, L. M., Reneman, R. S. \& Hoeks, A. P. The dicrotic notch as alternative time-reference point to measure local pulse wave velocity in the carotid artery by means of ultrasonography. J. Hypertens. 27, 2028-2035 (2009).

212. Mirault, T. et al. Carotid stiffness change over the cardiac cycle by ultrafast ultrasound imaging in healthy volunteers and vascular Ehlers-Danlos syndrome. J. Hypertens. 33, 1890-6- discussion 1896 (2015).

213. de Korte, C. L., Fekkes, S., Nederveen, A. J., Manniesing, R. \& Hansen, H. R. H. G. Review: Mechanical Characterization of Carotid Arteries and Atherosclerotic Plaques. IEEE Trans Ultrason Ferroelectr Freq Control 63, 1613-1623 (2016).

214. Couade, M. et al. Quantitative assessment of arterial wall biomechanical properties using shear wave imaging. Ultrasound Med Biol 36, 1662-1676 (2010).

215. Grotenhuis, H. B. et al. Validation and reproducibility of aortic pulse wave velocity as assessed with velocity-encoded MRI. J Magn Reson Imaging 30, 521-526 (2009).

216. Polak, J. F. et al. The Value of Carotid Artery Plaque and Intima-Media Thickness for Incident Cardiovascular Disease: The Multi-Ethnic Study of Atherosclerosis. Journal of the American Heart Association 2, (2013).

217. Iwakiri, T. et al. Usefulness of carotid intima-media thickness measurement as an indicator of generalized atherosclerosis: findings from autopsy analysis. Atherosclerosis 225, 359-362 (2012).

218. Crouse, J. R. et al. Effect of Rosuvastatin on Progression of Carotid Intima-Media Thickness in Low-Risk Individuals With Subclinical Atherosclerosis. JAMA 297, 1344-1353 (2007).

219. de Groot, E. et al. B-Mode Ultrasound Assessment of Pravastatin Treatment Effect on Carotid and Femoral Artery Walls and Its Correlations With Coronary Arteriographic Findings: A Report of the Regression Growth Evaluation Statin Study (REGRESS). J. Am. Coll. Cardiol. 31, 1561-1567 (1998).

220. Lorenz, M. W. et al. Carotid intima-media thickness progression to predict cardiovascular events in the general population (the PROG-IMT collaborative project): a meta-analysis of individual participant data. Lancet 379, 2053-2062 (2012).

221. Ravani, A. et al. Assessment and Relevance of Carotid Intima-Media Thickness (C-IMT) in Primary and Secondary Cardiovascular Prevention. Current Pharmaceutical Design 21, 1164 1171 (2015).

222. Zaid, M., Fujiyoshi, A., Kadota, A., Abbott, R. D. \& Miura, K. Coronary Artery Calcium and Carotid Artery Intima Media Thickness and Plaque: Clinical Use in Need of Clarification. $J$. Atheroscler. Thromb. 24, 227-239 (2017).

223. Iglesias del Sol, A., Bots, M. L., Grobbee, D. E., Hofman, A. \& Witteman, J. C. M. Carotid intimamedia thickness at different sites: relation to incident myocardial infarction. The Rotterdam Study. Eur. Heart J. 23, 934-940 (2002). 
224. O'Leary, D. H. \& Bots, M. L. Imaging of atherosclerosis: carotid intima-media thickness. Eur. Heart J. 31, 1682-1689 (2010).

225. Skilton, M. R. et al. Noninvasive measurement of carotid extra-media thickness: associations with cardiovascular risk factors and intima-media thickness. JACC Cardiovasc Imaging 2, 176-182 (2009).

226. Malik, S. et al. Impact of subclinical atherosclerosis on cardiovascular disease events in individuals with metabolic syndrome and diabetes: the multi-ethnic study of atherosclerosis. Diabetes Care 34, 2285-2290 (2011).

227. Boulos, N. M., Gardin, J. M., Malik, S., Postley, J. \& Wong, N. D. Carotid Plaque Characterization, Stenosis, and Intima-Media Thickness According to Age and Gender in a Large Registry Cohort. Am. J. Cardiol. 117, 1185-1191 (2016).

228. Agatston, A. S. et al. Quantification of coronary artery calcium using ultrafast computed tomography. J. Am. Coll. Cardiol. 15, 827-832 (1990).

229. Popovic, W. J., Brown, J. E. \& Adamson, J. W. The influence of thyroid hormones on in vitro erythropoiesis. Mediation by a receptor with beta adrenergic properties. J. Clin. Invest. 60, 907913 (1977).

230. Greenland, P. et al. ACCF/AHA 2007 clinical expert consensus document on coronary artery calcium scoring by computed tomography in global cardiovascular risk assessment and in evaluation of patients with chest pain: a report of the American College of Cardiology Foundation Clinical Expert Consensus Task Force (ACCF/AHA Writing Committee to Update the 2000 Expert Consensus Document on Electron Beam Computed Tomography). in 115, 402-426 (2007). Fujiyoshi, A. et al. Cross-Sectional Comparison of Coronary Artery Calcium Scores Between Caucasian Men in the United States and Japanese Men in JapanThe Multi-Ethnic Study of Atherosclerosis and the Shiga Epidemiological Study of Subclinical Atherosclerosis. Am J Epidemiol 180, 590-598 (2014).

232. Raggi, P., Callister, T. Q. \& Shaw, L. J. Progression of coronary artery calcium and risk of first myocardial infarction in patients receiving cholesterol-lowering therapy. Arteriosclerosis, Thrombosis, and Vascular Biology 24, 1272-1277 (2004).

233. Raggi, P. Cardiovascular disease: Coronary artery calcification predicts risk of CVD in patients with CKD. Nat Rev Nephrol 13, 324-326 (2017).

234. Yamamoto, H., Kitagawa, T. \& Kihara, Y. Clinical Implications of the Coronary Artery Calcium Score in Japanese Patients. J. Atheroscler. Thromb. 21, 1101-1108 (2014).

235. Krueger, T. et al. Vitamin K1 to slow vascular calcification in haemodialysis patients (VitaVasK trial): a rationale and study protocol. Nephrol. Dial. Transplant. 29, 1633-1638 (2014).

236. Vossen, L. M. et al. Menaquinone-7 Supplementation to Reduce Vascular Calcification in Patients with Coronary Artery Disease: Rationale and Study Protocol (VitaK-CAC Trial). Nutrients 7, 8905-8915 (2015).

237. Peeters, F. E. C. M. et al. Bicuspid Aortic Valve Stenosis and the Effect of Vitamin K2 on Calcification Using 18F-Sodium Fluoride Positron Emission Tomography/Magnetic Resonance: The BASIK2 Rationale and Trial Design. Nutrients 10, 386 (2018).

238. Geleijnse, J. M. et al. Dietary Intake of Menaquinone Is Associated with a Reduced Risk of Coronary Heart Disease: The Rotterdam Study. The Journal of Nutrition 134, 3100-3105 (2004). Brandenburg, V. M. et al. Slower Progress of Aortic Valve Calcification With Vitamin K Supplementation: Results From a Prospective Interventional Proof-of-Concept Study. Circulation 135, 2081-2083 (2017).

241. Chen, Z. et al. Does statins promote vascular calcification in chronic kidney disease? Eur. J. Clin. Invest. 47, 137-148 (2017).

242. Terry, J. G. et al. Effect of simvastatin $(80 \mathrm{mg})$ on coronary and abdominal aortic arterial calcium (from the coronary artery calcification treatment with zocor [CATZ] study). Am. J. Cardiol. 99, 1714-1717 (2007).

243. Criqui, M. H. et al. Calcium density of coronary artery plaque and risk of incident cardiovascular events. JAMA 311, 271-278 (2014).

244. Rumberger, J. A., Simons, D. B., Fitzpatrick, L. A., Sheedy, P. F. \& Schwartz, R. S. Coronary Artery Calcium Area by Electron-Beam Computed Tomography and Coronary Atherosclerotic Plaque Area. Circulation 92, 2157-2162 (1995)

245. Cho, I. et al. Prognostic implications of coronary artery calcium in the absence of coronary artery luminal narrowing. Atherosclerosis 262, 185-190 (2017). 


\section{Chapter 2}

246. Amann, K. Media calcification and intima calcification are distinct entities in chronic kidney disease. Clin J Am Soc Nephrol 3, 1599-1605 (2008).

247. Vos, A. et al. Risk factors for atherosclerotic and medial arterial calcification of the intracranial internal carotid artery. Atherosclerosis 276, 44-49 (2018).

248. Alluri, K. et al. Scoring of coronary artery calcium scans: History, assumptions, current limitations, and future directions. Atherosclerosis 239, 109-117 (2015).

249. Tanaka, R., Yoshioka, K., Takagi, H., Schuijf, J. D. \& Arakita, K. Novel developments in noninvasive imaging of peripheral arterial disease with CT: experience with state-of-the-art, ultrahigh-resolution CT and subtraction imaging. Clin Radiol (2018). doi:10.1016/j.crad.2018.03.002

250. Goldberger, Z. D. et al. Are changes in carotid intima-media thickness related to risk of nonfatal myocardial infarction? A critical review and meta-regression analysis. American Heart Journal 160, 701-714 (2010).

251. Criqui, M. H. et al. Coronary Artery Calcium Volume and Density: Potential Interactions and Overall Predictive Value: The Multi-Ethnic Study of Atherosclerosis. JACC Cardiovasc Imaging 10, 845-854 (2017).

252. Kim, K. P., Einstein, A. J. \& Berrington de González, A. Coronary artery calcification screening: estimated radiation dose and cancer risk. Archives of Internal Medicine 169, 1188-1194 (2009).

253. Bucerius, J., Dijkgraaf, I., Mottaghy, F. M. \& Schurgers, L. J. Target identification for the diagnosis and intervention of vulnerable atherosclerotic plaques beyond $18 \mathrm{~F}$-fluorodeoxyglucose positron emission tomography imaging: promising tracers on the horizon. Eur. J. Nucl. Med. Mol. Imaging 46, 251-265 (2019).

254. Blau, M., Ganatra, R. \& Bender, M. A. 18F-fluoride for bone imaging. Seminars in Nuclear Medicine 2, 31-37 (1972).

255. Dweck, M. R. et al. Coronary arterial 18F-sodium fluoride uptake: a novel marker of plaque biology. J. Am. Coll. Cardiol. 59, 1539-1548 (2012).

256. Joshi, N. V. et al. 18F-fluoride positron emission tomography for identification of ruptured and high-risk coronary atherosclerotic plaques: a prospective clinical trial. The Lancet 383, 705-713 (2014).

257. Irkle, A. et al. Identifying active vascular microcalcification by $<$ sup $>18</$ sup $>$ F-sodium fluoride positron emission tomography. Nature Communications 20156 6, 7495 (2015).

258. Hutcheson, J. D., Maldonado, N. \& Aikawa, E. Small entities with large impact: microcalcifications and atherosclerotic plaque vulnerability. Curr. Opin. Lipidol. 25, 327-332 (2014). 


\section{Chapter 3}

The role of medial vascular calcification on the development and progression of atherosclerosis

A.M.G. Jaminon, N. Rapp, R. van Gorp, M. Halder, P. Leenders, M. Bauwens, F Mottaghy, K. Reesink, R. Kramann, I. Dijkgraaf, C. Reutelingsperger, L.J. Schurgers

In Preparation 


\begin{abstract}
Background: Vascular calcification is becoming increasingly important in cardiovascular risk stratification. Vascular calcification is classified as either intimal or medial calcification, although clinically available methods do not allow this distinction yet. Suitable models that can study both medial and intimal calcification are scarce. We developed a novel mouse model to study the role of medial calcification on atherosclerosis development.

Methods: DBA/2 mice were treated with warfarin for either $0,1,2$ or 4 weeks to induce medial calcification. After warfarin treatment mice received a 12 week Western type diet (WTD) with or without a gain of function PCSK9 virus to induce LDLr knockdown. Before and after WTD, carotid-femoral pulse wave velocity (cfPWV) was measured. ${ }^{18} \mathrm{~F}-\mathrm{NaF}$ positron emission tomography (PET) spectra of the vasculature were taken. Hearts and the aorta were isolated and stained for Hematoxylin \& Eosin, Alizarin red, uncarboxylated matrix Gla protein and Elastica von Gieson.

Results: Warfarin treatment induced time dependently vascular, valvular and ectopic heart calcification. Warfarin induced extensive medial remodeling, such as extracellular matrix production, elastin damage and medial calcification. Additionally, we detected increased vessel stiffness and higher uptake of ${ }^{18} \mathrm{~F}-\mathrm{NaF}$ after warfarin treatment. Overexpression of PCSK9 in DBA/2 mice resulted in LDL receptor knockdown with a subsequent increase in plasma cholesterol levels. In LDLr knockdown animals, warfarin induced medial calcification resulted in accelerated atherosclerosis and concomitant increased intimal calcification.

Conclusion: To the best of our knowledge we report on the first mouse-model to study the effect of medial calcification on atherosclerosis development. We show that warfarin induces medial remodeling which results in accelerated atherosclerosis development. Our model provides the foundation to further study the impact of vascular remodeling on cardiovascular disease and develop new strategies for treatment.
\end{abstract}




\section{Introduction}

Vascular calcification is associated with an increase in CVD risk and affects about $90 \%$ of patients with symptomatic CVD ${ }^{1}$. Moreover, progression of arterial calcification ( $>15 \%$ annually) is associated with a significant increase in risk for myocardial infarction ${ }^{2}$. It has become evident that atherosclerotic calcification burden is a potent risk marker of cardiovascular events. Cardiovascular calcification is often present in elderly populations or patients with high atherosclerotic risk profile such as diabetes and chronic kidney disease. However, both chronic kidney disease (CKD) and diabetes patients are predominantly characterized by the development of medial calcification ${ }^{3,45}$. Recent meta-analyses indicated that over $70 \%$ of diabetic patients and up to $93 \%$ of CKD have arterial medial calcification ${ }^{6,7}$. Arterial medial calcification is associated with a 5- to 10-fold increased risk of CVD in CKD and diabetes patients ${ }^{1}$.

Vascular calcification can be classified as intimal and medial calcification, based on the anatomical site where it is detected ${ }^{8,9}$. Medial calcification, also known as Monckeberg sclerosis, is formed along the elastic fibers of the tunica media and can be found throughout the entire vascular tree ${ }^{10}$. Medial calcification is linked to vessel stiffness, hypertension and increased pulse wave velocity (PWV) ${ }^{11,12}$. Clinical events associated with medial calcification include heart failure, aneurysm formation and stroke through isolated systolic hypertension ${ }^{13-16}$. Data suggest that medial calcification is a stronger predictor for CVD events than intimal calcification in diabetes ${ }^{17,18}$ and CKD 19,20 .

Since medial calcification has been described to precede occurrence of atherosclerosis ${ }^{21}$ we aimed to investigate the role of medial calcification on atherosclerosis development in a novel developed experimental animal model.

\section{Methods}

\section{Animals}

DBA/2 mice were bought from Charles River Laboratories Inc ('sHertogenbosch, The Netherlands). Mice were kept in a temperature-controlled environment $\left(20^{\circ} \mathrm{C}\right)$ with a regular day \& night cycle. All animal experiments were conducted under a protocol approved by the ethics committee for animal experiments of Maastricht University.

Warfarin (a vitamin K antagonist; VKA) diet was prepared as described previously ${ }^{22}$. In brief, warfarin (3 mg/g; Sigma, Zwijndrecht, The Netherlands) supplemented with vitamin K1 (1.5 mg/g; Sigma, Zwijndrecht, The Netherlands) was mixed with normal chow diet (Arie Blok diets, Woerden, The Netherlands). Regular Western type diet (WTD) was bought directly from the supplier $(0.25 \%$ cholesterol; Arie Blok diets, Woerden, The Netherlands). pAAV/D374Y-hPCSK9 (PCSK9) overexpressing virus was obtained from the Viral Core Facility (Charité Universitäts Medizin, Berlin, Germany). PCSK9 virus was diluted in $0.9 \%$ Saline and stored at $-80^{\circ} \mathrm{C}$ until use.

Experimental animals (10-12 weeks old males) were weighed, tonometry was performed and subsequently mice were placed on warfarin diet for either 0 week (control), 1, 2 or 4 weeks (Figure 1). After warfarin diet the animals underwent a second tonometry measurement after which they were placed on a WTD for 12 weeks. One day before sacrifice animals received a third and last tonometry measurement. For the pilot 
experiment, animals were treated similarly except after warfarin diet, mice were injected IV with PCSK9 virus $\left(5^{*} 10^{10}\right.$ particles/ mouse) after which they were placed on a WTD for 12 weeks.

At sacrifice, blood was collected in $3.2 \%$ sodium citrate by vena cava puncture and the aorta was flushed with $100 \mu \mathrm{M}$ sodium nitroprusside in phosphate buffered saline (PBS). The aortic arch, its main branches as well as other organs, were excised and fixated in $1 \%$ paraformaldehyde overnight and embedded in paraffin. Some mice $(n=6)$ received ${ }^{18} \mathrm{~F}-\mathrm{NaF}$ intravenously (IV) to image active vascular calcification via micro positron emission tomography - computed tomography ( $\mu$ PET-CT) scanning. Aortic arch and aortic root were sectioned and stained for hematoxylin and eosin (HE), alizarin red (AR), Elastica von Gieson (EvG) and uncarboxylated matrix Gla-protein (ucMGP).

\section{Plasma cholesterol determination}

Total cholesterol (Horiba Medical, Montpellier, France) measurements were performed as described in manufacturer's manual.

$\mu P E T^{18} \mathrm{~F}-\mathrm{NaF}$

Animals were starved 2 hours before ${ }^{18} \mathrm{~F}-\mathrm{NaF}$ injection. Mice were anaesthetized and $\pm 10 \mathrm{MBq}{ }^{18} \mathrm{~F}-\mathrm{NaF}$ was injection IV after which mice were left to wake up. ${ }^{18} \mathrm{~F}-\mathrm{NaF}$ was circulated for 30 minutes before the animals were sacrificed as described above. Aortic arches were isolated and imaged with $\mu$ PET.

\section{Pulse wave velocity}

Animals were anaesthetized and the left cervix and groin were shaved. ECG electrodes were connected, and the first tonometer probe was placed on the cervix (left carotid) while the second probe was placed on the groin (left femoral artery). After a stable heartbeat signal was obtained the measurement was started and the distance between the cervix and groin was measured using a caliper.

\section{Histology and histochemistry}

$5 \mu \mathrm{M}$ serial sections were cut from paraffin embedded aortic arches and aortic roots and stained with Alizarin Red (AR), hematoxylin and eosin (HE). AR positivity was determined using FIJI software (v2.0.0-RC-69) and normalized against total aortic area. Atherosclerotic plaque burden was analyzed on HE stains and normalized against total arch area using FIJI software (v2.0.0-RC-69). Total atherosclerotic burden was measured in the aortic arch and its major branching points. Atherosclerotic calcification was determined as AR positivity inside plaques.

\section{Western blotting}

Livers were homogenized with glass beads and a bead beater in RIPA buffer (89900, Thermo Scientific) containing MS-SAFE (MSSAFE-5VL, Sigma). Protein concentrations were determined using microBCA assay (23235, Thermo Scientific) and lysates were separated on 7.5\% Mini-PROTEAN TGX Precast Protein Gels (456-1024, BioRad). Samples were transferred to a nitrocellulose membrane (BioRad) and incubated overnight with anti-LDLr (3839-100, Biovision) and anti-GAPDH (MAB374, Millipore). Protein was detected using HRP-conjugated secondary antibodies (antichicken: ab97135, Abcam; anti-rabbit: 7074S, Cell Signalling) and visualized by 
enhanced chemiluminescence (Pierce ECL Western Blotting Substrate, Thermo Scientific).

\section{Immunohistochemistry}

Antigen retrieval was performed by heating samples in target retrieval solution (Dako, Heverlee, Belgium). Subsequently, tissues were blocked and incubated with primary antibody overnight as described: ucMGP (1:400; IDS, Boldon, UK). After overnight incubation, sections were incubated for 1 hour with HRP-conjugated secondary antibody (p0447; Dako) and detected with NovaRED (Vector Labs, Amsterdam, the Netherlands).

Elastin fibers were detected using Elastica von Gieson staining. In brief, tissues were stained in Lawson solution (Klinipath, Olen, Belgium) and differentiated in 70\% ethanol. Tissues were counterstained for collagen and connective tissue in von Gieson solution.

Images were captured using a DM400 microscope (Leica, Eindhoven, the Netherlands) and imaging software (LAS V, Eindhoven, the Netherlands).

\section{Statistical analysis}

All values are shown as mean \pm SD unless stated differently. Statistical analysis was performed by 1- way ANOVA with Bonferroni post hoc test using PRISM software (GraphPad). Values of $\mathrm{P}<0.05$ were considered statistically significant.

\section{Results}

In this study we exposed animals to a VKA antagonist diet (warfarin) to induce time dependently medial calcification ( 1 week, 2 weeks and 4 weeks warfarin treatment). A schematic overview of the experimental design is displayed in Figure 1A.
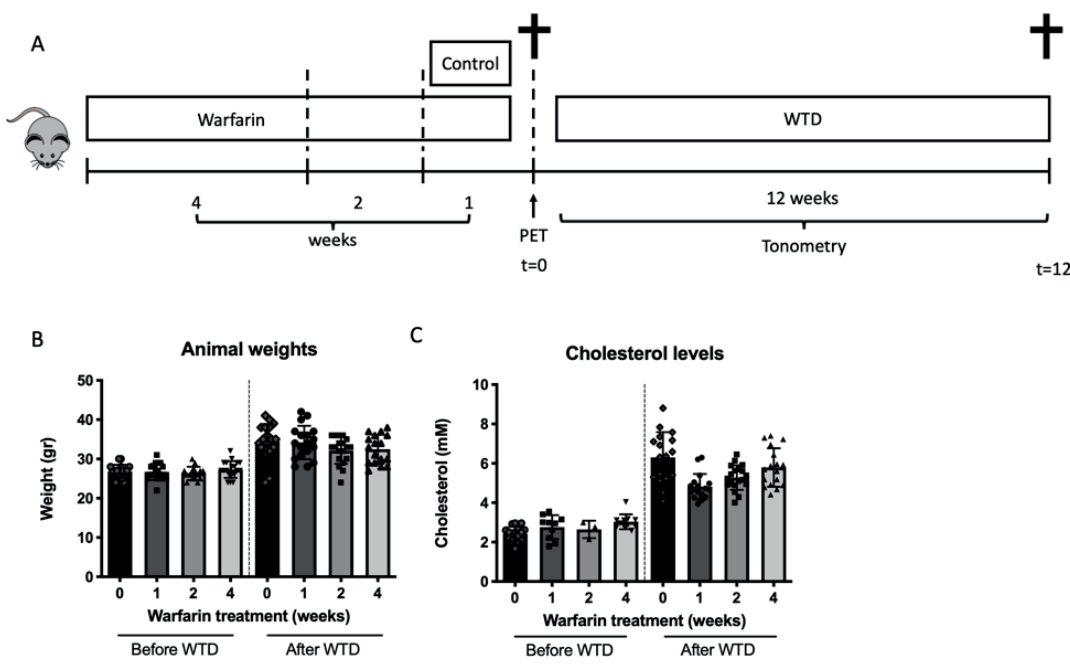

Figure 1. Baseline characteristics. (A) Schematic overview of the experimental design of the study. (B) Bodyweights of DBA $/ 2$ mice at $\mathrm{t}=0$ and $\mathrm{t}=12$ weeks of WTD diet. (C) Cholesterol levels of DBA $/ 2$ mice at $\mathrm{t}=0$ and $\mathrm{t}=12$ weeks of WTD. 


\section{Baseline characteristics}

Total bodyweights of mice at $\mathrm{t}=0$ and $\mathrm{t}=12$ were not changed by exposure to different durations of warfarin (Figure 1B). As expected, weight of animals increased after WTD (mean $33.3 \pm 4.0$ gr), as compared to before WTD (mean $26.8 \pm 1.9$ gr; Figure 1B). Additionally, cholesterol levels after $\mathrm{t}=0$ and $\mathrm{t}=12$ were not altered between control and warfarin treated animals (Figure 1C). Cholesterol levels were significantly higher when comparing the animals before (mean $2.7 \mathrm{mM}$ ) and after WTD (mean $5.5 \mathrm{mM}$; $\mathrm{p}<0.0001$; Figure 1C).

Atherosclerosis does normally not develop in rodents ${ }^{23}$, and during warfarin treatment we could not detect atherosclerotic plaques (supplemental Figure 1).

\section{Cardiovascular calcification}

Medial calcification of the aorta as quantified by AR staining was significantly increased in the animals in the 4-week warfarin treated group (Figures 2A and 2B) compared to animals treated for 0,1 and 2 weeks of warfarin $(0$ and 1 week, $p<0.0001$; 2 weeks, $\mathrm{p}<0.001)$. Medial calcification was predominantly found in the ascending aorta, close to the aortic root. Furthermore, after WTD medial calcification was significantly increased in animals that received warfarin for only 1 or 2 weeks as compared to no warfarin pretreatment. Additionally, aortic valve calcification was significantly increased in the 4 week warfarin group ( $p=0.026$; Figures $2 \mathrm{C}$ and $2 \mathrm{D})$. Noticeably, calcification did not occur in the luminal/ leaflet region but was found at the border of the aortic valve area (black arrow, Figure 2C).

$\mathrm{DBA} / 2$ mice are also prone to develop calcification of the heart, more specifically dystrophic cardiac calcifications ${ }^{24}$. Therefore, dystrophic calcification of the heart was determined before and after WTD. Warfarin treated animals displayed increased calcification of the heart compared to control treated animals ( $p=0.002$; Figure $3 \mathrm{~A})$.

A

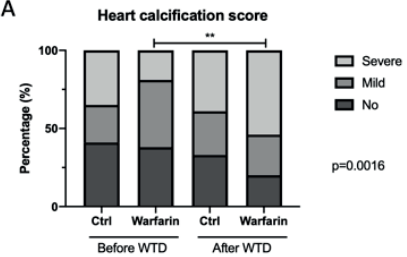

C

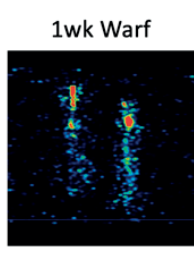

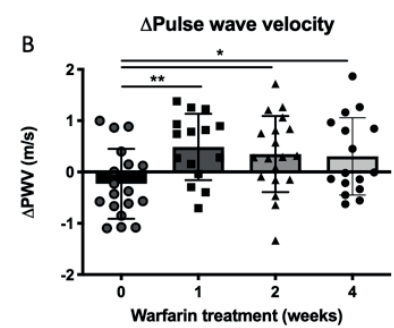

D

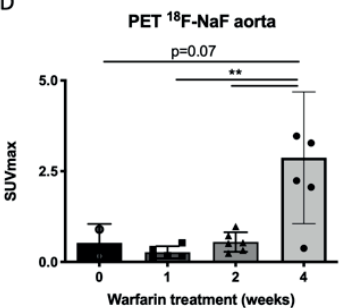


Figure 2. Heart calcification, pulse wave velocity and ${ }^{18} \mathrm{~F}-\mathrm{NaF}$ uptake. (A) Heart calcification score of animals at $\mathrm{t}=0$ and $\mathrm{t}=12$ weeks. A significant increase in heart calcification was found in animals treated with VKA. (B) Delta pulse wave velocity (PWV) comparing $\mathrm{t}=0$ with $\mathrm{t}=12$ weeks of WTD. All animals on WTD showed a significant increase in pulse wave velocity when challenged with a VKA diets, while animals on a control diet did not increase PWV. (C) ${ }^{18} \mathrm{~F}-\mathrm{NaF}$ PET scans of aortic arches. (D) Quantification of ${ }^{18} \mathrm{~F}-\mathrm{NaF}$ signal. There was a significant increase in ${ }^{18} \mathrm{~F}-\mathrm{NaF}$ uptake in animals treated for 4 weeks with VKA. *p $<0.05$; $* * \mathrm{p}<0.01$

\section{Pulse wave velocity and ${ }^{18} \mathrm{~F}-\mathrm{NaF}$ uptake}

Medial calcification is linked to vessel wall stiffness and therefore we measured the carotid-femoral PWV (cfPWV). PWV was assessed before and after WTD and is displayed as delta value $(\triangle \mathrm{PWV}=(\mathrm{PWV}$ at $\mathrm{t}=\mathrm{end})-(\mathrm{PWV}$ at $\mathrm{t}=$ Before $\mathrm{WTD}))$. Priming the vasculature for 1,2 and 4 weeks of warfarin showed a significant increase in cfPWV compared to control (no warfarin treatment; $p<0.01$; Figure $3 B$ ).

Since warfarin inhibits the activation of vitamin $\mathrm{K}$ dependent proteins, and thus induces calcification by impaired calcification inhibition, we measured calcification activity using the PET tracer ${ }^{18} \mathrm{~F}-\mathrm{NaF} .{ }^{18} \mathrm{~F}-\mathrm{NaF}$ uptake is measured as an increase in SUVmax. ${ }^{18} \mathrm{~F}-\mathrm{NaF}$ uptake in the 4-week warfarin treatment was significantly increased as compared to the other treatment groups ( $\mathrm{p}<0.01$; Figures $3 \mathrm{C}$ and $3 \mathrm{D})$.
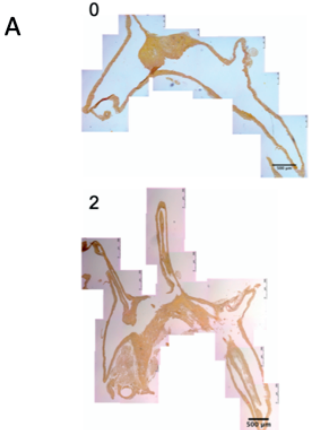

C
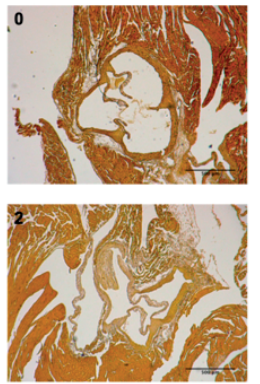
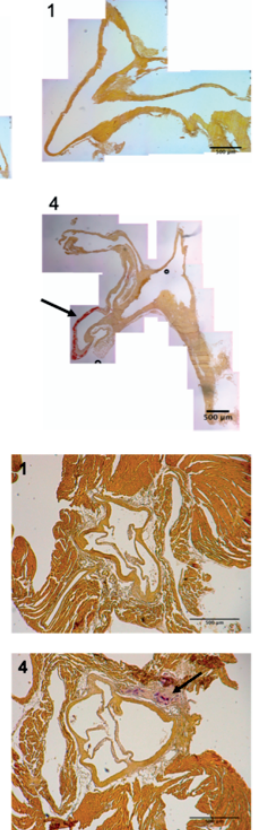

B

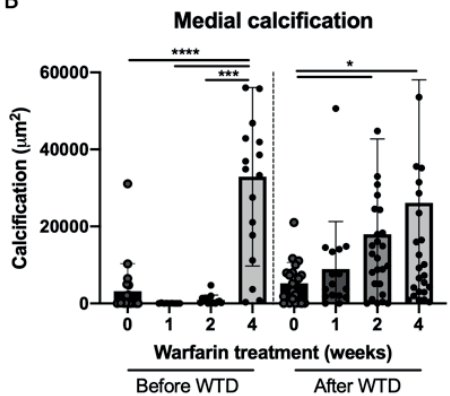

D

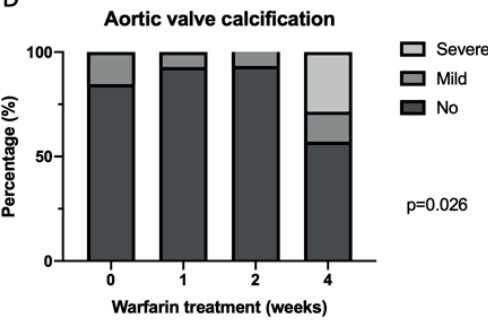

Figure 3. Heart calcification. (A) Representative images of the aortic arch and stained for alizarin red (AR). (B) Quantification of AR signal. At $t=0$ a significant increase in AR positivity was found in the 4 week VKA groups. At $\mathrm{t}=12$ weeks both 2 and 4 weeks of VKA treatment significantly increased AR positivity. (C) Representative images of the aortic root area stained for AR. (D) calcification score of AR positivity in the aortic root area. A significant increase was found in the 4 weeks VKA group. ${ }^{*} p<0.05 ; * * * p<0.001$; $* * * * \mathrm{p}<0.0001$ 


\section{PCSK9 pAAV gain of function to induce atherosclerosis}

DBA/2 mice do not develop atherosclerosis. Therefore, to provoke atherosclerosis, we employed PCSK9 overexpressing virus (pAAV) to induce LDL receptor (LDLr) knockdown, followed by 12 weeks of WTD ${ }^{25}$. Virus efficacy was determined by protein expression of the LDL receptor in liver lysates. LDLr knockdown was achieved up to $95 \%$ compared to control animals, were no virus was injected (Figure 4A and supplemental Figure 2B). Additionally, plasma cholesterol levels significantly increased after PCSK9 overexpression (mean $31.2 \pm 9.1 \mathrm{mM}$ ) compared to control animals on WTD (mean $5.6 \pm 1.2 \mathrm{mM})$ for the same duration $(\mathrm{p}<0.0001$; Figure $4 \mathrm{~B}$ and Table 1).
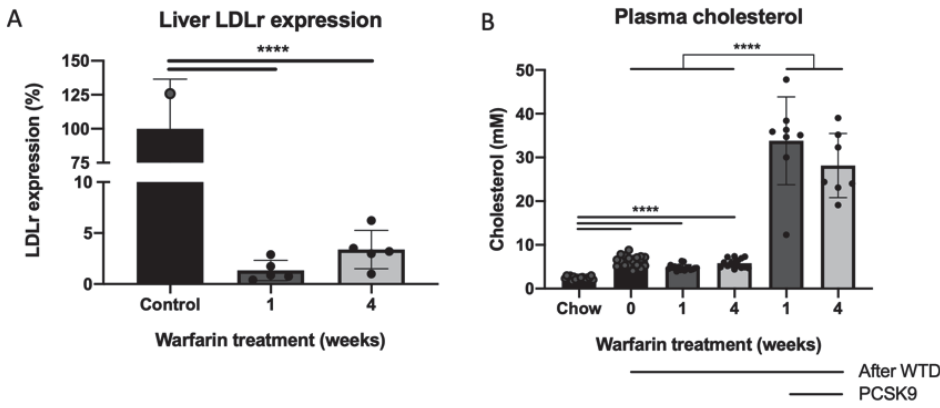

Figure 4. Atherosclerosis induction. (A) LDL receptor expression was significantly decreased in liver lysates of mice that received PCSK9 overexpressing virus (1 and 4 weeks VKA groups). (B) plasma cholesterol levels before and after WTD and with and without the PCSK9 virus. WTD significantly increased the plasma cholesterol levels. PCSK9 overexpression significantly increased plasma cholesterol compared to WTD only. $* * * * \mathrm{p}<0.0001$

Table 1. Animal weight and Cholesterol levels.

\begin{tabular}{|c|c|c|c|c|c|c|c|c|c|}
\hline & & \multicolumn{2}{|c|}{$t=0$} & \multicolumn{2}{|c|}{$t=4$} & \multicolumn{2}{|c|}{$t=8$} & \multicolumn{2}{|c|}{$t=12$} \\
\hline & & Average & SD & Average & SD & Average & SD & Average & SD \\
\hline \multirow{2}{*}{ Weight (g) } & $1 \mathrm{wk} \mathrm{W}$ & 24,8 & 2,4 & 28,5 & 3,4 & 31,4 & 3,4 & 31,6 & 3,3 \\
\hline & 4 wk W & 28,2 & 3,7 & 30,1 & 5,5 & 33,2 & 5,8 & 34,1 & 6,0 \\
\hline \multirow{2}{*}{ Cholesterol (mM) } & $1 \mathrm{wk} \mathrm{W}$ & & & 33,8 & 7,2 & 39,3 & 8,3 & 33,8 & 10,0 \\
\hline & 4 wk W & & & 43,8 & 4,7 & 25,9 & 7,4 & 28,1 & 7,3 \\
\hline
\end{tabular}

\section{Atherosclerosis development after medial calcification.}

As described above, DBA/2 mice treated with warfarin develop medial calcification without atherosclerosis. After different time durations of warfarin treatment, DBA/2 mice were injected with PCSK9 virus and treated for 12 weeks with WTD. Warfarin treated animals developed extensive medial calcification (Figures 5A and 5B) with simultaneously also atherosclerotic plaque calcification, that was significantly increased compared to control animals ( $<<0.0001$; Figures $5 \mathrm{C}$ and 5D). 
Additionally, warfarin duration was associated with increased development of intimal calcification $(\mathrm{p}<0.01$; Figures $5 \mathrm{C}$ and $5 \mathrm{D})$.
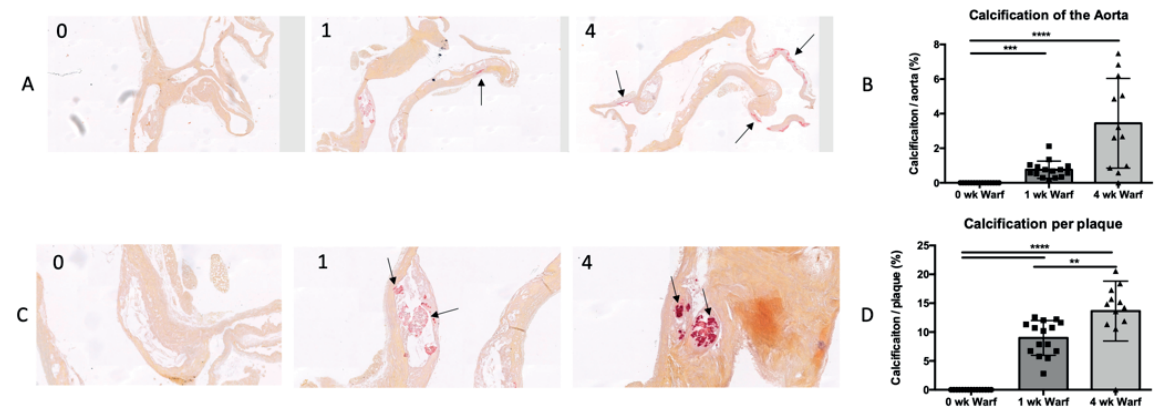

Figure 5. Atherosclerosis development after vascular calcification induction. (A) Representative images of aortic arches and stained for AR. Animals were treated for 0, 1 and 4 weeks of VKA (4x magnification). (B) Quantification of AR signal. Both 1 week and 4 weeks of VKA use significantly increased the AR signal. (C) representative images of atherosclerotic plaques stained for AR. Animals were treated with VKA for 0, 1 and 4 weeks (10x magnification). (D) Quantification of AR signal normalized for plaque area. Both 1 week and 4 weeks of VKA treatment induced a significant increase in plaque calcification. ${ }^{* *} \mathrm{p}<0.01 ; * * * \mathrm{p}<0.001$; $* * * * \mathrm{p}<0.0001$

\section{ucMGP and medial remodeling}

UcMGP positivity was found in close proximity of intimal calcification (Figure 6A, panel A2 and A3) and at areas of medial calcification (Figure 6A, panel A4 and A5). Warfarin treated animals showed significantly more ucMGP positivity compared to control animals ( $p<0.0001$; Figure 6B).

Medial remodeling is often found at shoulder regions of atherosclerotic plaques (Figure 6C, panel $\mathrm{C} 4$ and $\mathrm{C} 5$ ). Warfarin treatment induced significantly more elastin damage in the medial layer of the vessel wall compared to control treatment $(p<0.01$; Figure 6D). Elastin fibers underneath atherosclerotic plaques were often thinner (Figure 6C, panel $\mathrm{C} 2$ ) and showed a higher degree of debranching (Figure 6C, panel C5). Furthermore, elastin breaks were found more frequently underneath atherosclerotic plaques (Figure 6C, panel C3) compared to areas without atherosclerotic plaques (Figure $6 \mathrm{C}$, panel $\mathrm{C} 1$ ). Additionally, elastin breaks were found at areas with medial calcification (Figure 6C, panel C6). 


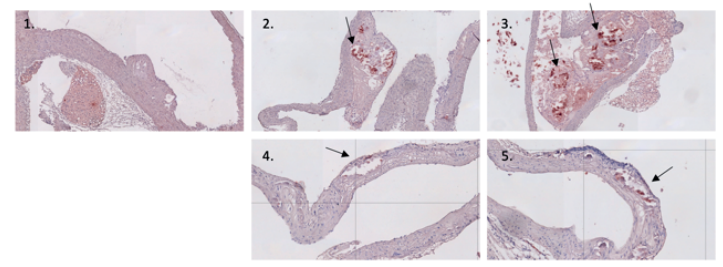

C

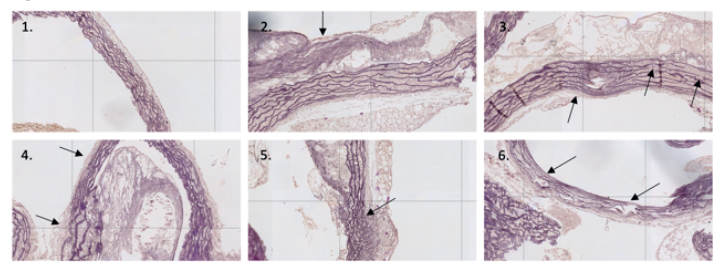

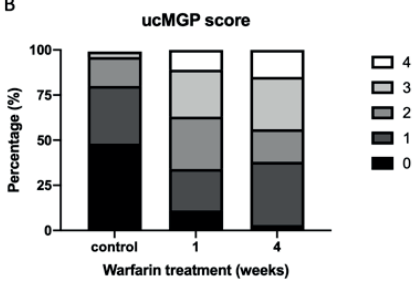

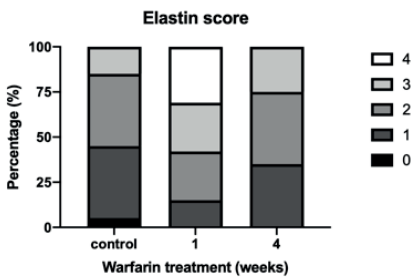

Figure 6. ucMGP and elastin staining. (A) Representative images of areas of ucMGP positivity. 1.0 week VKA animal with no ucMGP positivity. 2. 1 week VKA animal with ucMGP positivity inside the atherosclerotic plaque. 3. 4 week VKA animal with ucMGP positivity inside the atherosclerotic plaque. 4. \& 5. Medial calcification show colocalisation with ucMGP positivity. (B) ucMGP positivity score was significantly increased in the 4 week VKA group compared to control $p<0.0001$. (C) Representative images of Elastica von Gieson staining. 1. Vessel wall without atherosclerotic plaque. 2. Atherosclerotic plaque. 3. Elastin breaks underneath atherosclerotic plaques. 4. Medial remodeling at paque shoulder regions. 5. Debranching at plaque shoulder regions. 6. Medial calcification. (D) Elastin damage score. A significant increase in elastin damage was found in animals treated with VKA $\mathrm{p}<0.01$.

\section{Discussion}

In this paper, we describe the first experimental animal model to investigate effects of vascular medial remodeling and calcification on development of atherosclerosis. We show that short term VKA treatment has major consequences on the long term, both in development of medial calcification, aortic valve calcification and increased vascular stiffness. Additionally, even short term VKA treatment resulted in significantly increased intimal calcification. Our data indicate that VKA induces vascular remodeling that is not visible with current imaging modalities, but which results in increased progression of atherosclerosis and intimal calcification.

We and others have shown that VKA treatment induces development of both medial ${ }^{22}$ and intimal calcification ${ }^{26,27}$. Warfarin has been extensively used as inducer for medial calcification ${ }^{28}$. C57bl/6 mice however, do not develop medial calcification when treated with warfarin. Even 5/6 nephrectomy, a well-established vascular calcification model in rats, did not induce significant vascular calcification in $\mathrm{C} 57 \mathrm{bl} / 6$ mice ${ }^{29}$. Moreover, Apoe deficient mice on a C57bl/6 background are known to develop atherosclerosis when fed a western type diet, yet hardly develop significant medial calcification ${ }^{26}$. DBA/2 mice are known for developing ectopic calcification, which is due to a defect in the $A b c c 6$ gene and low plasma magnesium levels ${ }^{24}$. DBA/2 mice develop spontaneous ectopic heart calcification ${ }^{22}$ which is concurrent with our results. We observed that almost $70 \%$ of animals displayed signs of calcification of the heart which was further enhanced by VKA treatment. 
Since DBA/2 mice do not develop atherosclerosis, we applied a recently developed LDLr knockdown using PCSK9 gain of function virus ${ }^{25}$. In animals on a WTD but with no LDLr knockdown, cholesterol levels only increased slightly compared to chow-fed mouse plasma cholesterol levels. Animals that received the virus displayed high cholesterol plasma levels that remained elevated for the whole duration of the experiment. It has been shown that LDLr knockdown using PCSK9 gain of function results in atherosclerotic calcification, however without signs of medial calcification ${ }^{30}$. Therefore, we developed an experimental animal model sensitive to develop both medial calcification and atherosclerosis.

Literature that dates back to the time before non-invasive imaging, reported that atherosclerotic plaques develop in areas of medial degradation or calcification ${ }^{21}$. With the introduction of non-invasive imaging detailed information about the precise location of calcification is lost. Current multi slice CT cannot differentiate between medial and intimal calcification. Most literature reports on a relation between progression of lesion size and plaque calcification ${ }^{31,32}$. In our study we show that medial calcification present prior to atherosclerosis results in progression of atherosclerotic plaque calcification. We hypothesize that vitamin $\mathrm{K}$ deficiency can lead to detrimental medial remodeling causing elastin damage forming the nidus for calcification. Moreover, we show that extensive medial remodeling contributes to accelerated atherosclerosis.

${ }^{18} \mathrm{~F}-\mathrm{NaF}$ PET scanning has recently been introduced as novel imaging modality for vascular calcification ${ }^{33}$ and is used to detect active calcification with relatively high specificity ${ }^{34}$. In our study we examined uptake of ${ }^{18} \mathrm{~F}-\mathrm{NaF}$ to confirm active mineralisation. We found a significant increase in ${ }^{18} \mathrm{~F}-\mathrm{NaF}$ PET signal in warfarin treated animals, indicative for active calcification. Measurement of active calcification by ${ }^{18} \mathrm{~F}$ $\mathrm{NaF}$ is considered complementary to $\mathrm{CT}$ and predicts progression of calcification ${ }^{35,36}$. Furthermore, ${ }^{18} \mathrm{~F}-\mathrm{NaF}$ detects high risk lesions and future cardiovascular events more accurately ${ }^{37,38}$. The presence of both medial and intimal vascular calcification was confirmed by histochemical staining. ${ }^{18} \mathrm{~F}-\mathrm{NaF}$ positivity was found to colocalize in areas of calcification positive stain in the aortic arch. It is known that presence of vascular calcification increases the risk for cardiovascular morbidity and mortality ${ }^{1}$. We also noticed that VKA increased aortic valve calcification. Aortic valve calcification is common in the ageing population and has become a substantial disease burden in the Western world ${ }^{39}$. The precise pathophysiology of calcific aortic valve stenosis is still unclear but use of VKA is known to accelerate this process ${ }^{40-42}$. Moreover, aortic valve calcification is associated with increased cardiovascular events and mortality ${ }^{43}$.

\section{Conclusion}

Medial calcification represents an underestimated risk factor for cardiovascular disease. In this study we developed a mouse model that develops both medial calcification and atherosclerosis. We show that VKA use, dose dependently increases vascular, valvular and ectopic heart calcification. Additionally, short term VKA treatment increases vessel stiffness and elastic fiber damage resulting in accelerated atherosclerosis. Our research opens new avenues to study the impact of vascular remodeling on atherosclerosis and whether targeting medial calcification results in reduced cardiovascular disease. 


\section{References}

1. Rennenberg, R. J. M. W. et al. Vascular calcifications as a marker of increased cardiovascular risk: a meta-analysis. Vasc Health Risk Manag 5, 185-197 (2009).

2. Raggi, P., Callister, T. Q. \& Shaw, L. J. Progression of coronary artery calcium and risk of first myocardial infarction in patients receiving cholesterol-lowering therapy. Arteriosclerosis, Thrombosis, and Vascular Biology 24, 1272-1277 (2004).

3. Niskanen, L., Siitonen, O., Suhonen, M. \& Uusitupa, M. I. Medial artery calcification predicts cardiovascular mortality in patients with NIDDM. Diabetes Care 17, 1252-1256 (1994).

4. Garimella, P. S. \& Sarnak, M. J. Cardiovascular disease in CKD in 2012: moving forward, slowly but surely. Nat Rev Nephrol 9, 69-70 (2013).

5. Durham, A. L., Speer, M. Y., Scatena, M., Giachelli, C. M. \& Shanahan, C. M. Role of smooth muscle cells in vascular calcification: implications in atherosclerosis and arterial stiffness. Cardiovasc. Res. 114, 590-600 (2018).

6. Kramer, C. K. et al. Coronary artery calcium score prediction of all cause mortality and cardiovascular events in people with type 2 diabetes: systematic review and meta-analysis. $B M J$ 346, f1654-f1654 (2013).

7. Wang, X.-R., Zhang, J.-J., Xu, X.-X. \& Wu, Y.-G. Prevalence of coronary artery calcification and its association with mortality, cardiovascular events in patients with chronic kidney disease: a systematic review and meta-analysis. 41, 244-256 (2019).

8. Amann, K. Media calcification and intima calcification are distinct entities in chronic kidney disease. Clin J Am Soc Nephrol 3, 1599-1605 (2008).

9. Willems, B. A. G., Vermeer, C., Reutelingsperger, C. P. M. \& Schurgers, L. J. The realm of vitamin K dependent proteins: Shifting from coagulation toward calcification. Mol Nutr Food Res 58, 1620 1635 (2014).

10. Mizobuchi, M., Towler, D. \& Slatopolsky, E. Vascular calcification: the killer of patients with chronic kidney disease. J. Am. Soc. Nephrol. 20, 1453-1464 (2009).

11. Neven, E. \& D'Haese, P. C. Vascular calcification in chronic renal failure: what have we learned from animal studies? Circ. Res. 108, 249-264 (2011).

12. Chow, B. \& Rabkin, S. W. The relationship between arterial stiffness and heart failure with preserved ejection fraction: a systemic meta-analysis. Heart Fail Rev 20, 291-303 (2015).

13. Vos, A. et al. Risk factors for atherosclerotic and medial arterial calcification of the intracranial internal carotid artery. Atherosclerosis 276, 44-49 (2018).

14. Petsophonsakul, P. et al. Role of Vascular Smooth Muscle Cell Phenotypic Switching and Calcification in Aortic Aneurysm Formation. Arteriosclerosis, Thrombosis, and Vascular Biology 27, ATVBAHA119312787 (2019).

15. Kaess, B. M. et al. Aortic stiffness, blood pressure progression, and incident hypertension. JAMA 308, 875-881 (2012).

16. Lanzer, P. et al. Medial vascular calcification revisited: review and perspectives. Eur. Heart J. 35, $1515-1525$ (2014).

17. Lehto, S., Niskanen, L., Suhonen, M., Rönnemaa, T. \& Laakso, M. Medial artery calcification. A neglected harbinger of cardiovascular complications in non-insulin-dependent diabetes mellitus. Arteriosclerosis, Thrombosis, and Vascular Biology 16, 978-983 (1996).

18. Everhart, J. E., Pettitt, D. J., Knowler, W. C., Rose, F. A. \& Bennett, P. H. Medial arterial calcification and its association with mortality and complications of diabetes. Diabetologia 31, 1623 (1988).

19. Toussaint, N. D. \& Kerr, P. G. Vascular calcification and arterial stiffness in chronic kidney disease: implications and management. Nephrology (Carlton) 12, 500-509 (2007).

20. Disthabanchong, S., Vipattawat, K., Phakdeekitcharoen, B., Kitiyakara, C. \& Sumethkul, V. Abdominal aorta and pelvic artery calcifications on plain radiographs may predict mortality in chronic kidney disease, hemodialysis and renal transplantation. Int Urol Nephrol 50, 355-364 (2018).

21. Blumenthal, H. T., Lansing, A. I. \& Wheeler, P. A. Calcification of the Media of the Human Aorta and Its Relation to Intimal Arteriosclerosis, Ageing and Disease. The American Journal of Pathology 20, 665-687 (1944).

22. Kruger, T. et al. Warfarin Induces Cardiovascular Damage in Mice. Arteriosclerosis, Thrombosis, and Vascular Biology 33, 2618-2624 (2013).

23. Paigen, B., Ishida, B. Y., Verstuyft, J., Winters, R. B. \& Albee, D. Atherosclerosis susceptibility differences among progenitors of recombinant inbred strains of mice. Arteriosclerosis 10, 316-323 (1990). 
24. van den Broek, F. A. \& Beynen, A. C. The influence of dietary phosphorus and magnesium concentrations on the calcium content of heart and kidneys of DBA/2 and NMRI mice. Lab. Anim. 32, 483-491 (1998).

25. Bjørklund, M. M. et al. Induction of atherosclerosis in mice and hamsters without germline genetic engineering. Circ. Res. 114, 1684-1689 (2014).

26. Schurgers, L. J. et al. Vitamin K-antagonists accelerate atherosclerotic calcification and induce a vulnerable plaque phenotype. PLoS ONE 7, e43229 (2012).

27. Chatrou, M. L. L., Winckers, K., Hackeng, T. M., Reutelingsperger, C. P. \& Schurgers, L. J. Vascular calcification: The price to pay for anticoagulation therapy with vitamin K-antagonists. YBLRE 26, 155-166 (2012).

28. Price, P. A., Faus, S. A. \& Williamson, M. K. Warfarin Causes Rapid Calcification of the Elastic Lamellae in Rat Arteries and Heart Valves. Arteriosclerosis, Thrombosis, and Vascular Biology 18, 1400-1407 (1998).

29. Shobeiri, N., Adams, M. A. \& Holden, R. M. Vascular calcification in animal models of CKD: A review. Am. J. Nephrol. 31, 471-481 (2010).

30. Goettsch, C. et al. A single injection of gain-of-function mutant PCSK9 adeno-associated virus vector induces cardiovascular calcification in mice with no genetic modification. Atherosclerosis 251, 109-118 (2016).

31. Win, T. T. et al. Apixaban versus warfarin in evaluation of progression of atherosclerotic and calcified plaques (prospective randomized trial). American Heart Journal 212, 129-133 (2019).

32. Lee, J. et al. Randomized trial of rivaroxaban versus warfarin in the evaluation of progression of coronary atherosclerosis. American Heart Journal 206, 127-130 (2018).

33. Bucerius, J., Dijkgraaf, I., Mottaghy, F. M. \& Schurgers, L. J. Target identification for the diagnosis and intervention of vulnerable atherosclerotic plaques beyond $18 \mathrm{~F}$-fluorodeoxyglucose positron emission tomography imaging: promising tracers on the horizon. Eur. J. Nucl. Med. Mol. Imaging 46, 251-265 (2019).

34. Dweck, M. R. et al. Coronary arterial $18 \mathrm{~F}$-sodium fluoride uptake: a novel marker of plaque biology. J. Am. Coll. Cardiol. 59, 1539-1548 (2012).

35. Pawade, T. A., Newby, D. E. \& Dweck, M. R. Calcification in Aortic Stenosis: The Skeleton Key. J. Am. Coll. Cardiol. 66, 561-577 (2015).

36. Nakahara, T., Narula, J., Fox, J. J., Jinzaki, M. \& Strauss, H. W. Temporal relationship between $18 \mathrm{~F}$-sodium fluoride uptake in the abdominal aorta and evolution of CT-verified vascular calcification. J Nucl Cardiol 15, 827-10 (2019).

37. Irkle, A. et al. Identifying active vascular microcalcification by $<$ sup $>18</$ sup $>$ F-sodium fluoride positron emission tomography. Nature Communications 2015 6 6,7495 (2015).

38. Kitagawa, T. et al. Predictive Value of 18F-Sodium Fluoride Positron Emission Tomography in Detecting High-Risk Coronary Artery Disease in Combination With Computed Tomography. Journal of the American Heart Association 7, e010224 (2018).

39. Bonow, R. O. \& Greenland, P. Population-wide trends in aortic stenosis incidence and outcomes. Circulation 131, 969-971 (2015).

40. Schurgers, L. J., Aebert, H., Vermeer, C., Bültmann, B. \& Janzen, J. Oral anticoagulant treatment: friend or foe in cardiovascular disease? Blood 104, 3231-3232 (2004).

41. Koos, R. et al. Relation of oral anticoagulation to cardiac valvular and coronary calcium assessed by multislice spiral computed tomography. Am. J. Cardiol. 96, 747-749 (2005).

42. Peeters, F. E. C. M. et al. Calcific aortic valve stenosis: hard disease in the heart: A biomolecular approach towards diagnosis and treatment. Eur. Heart J. 39, 2618-2624 (2018).

43. Owens, D. S. et al. Aortic valve calcium independently predicts coronary and cardiovascular events in a primary prevention population. JACC Cardiovasc Imaging 5, 619-625 (2012). 


\section{Supplemental material}
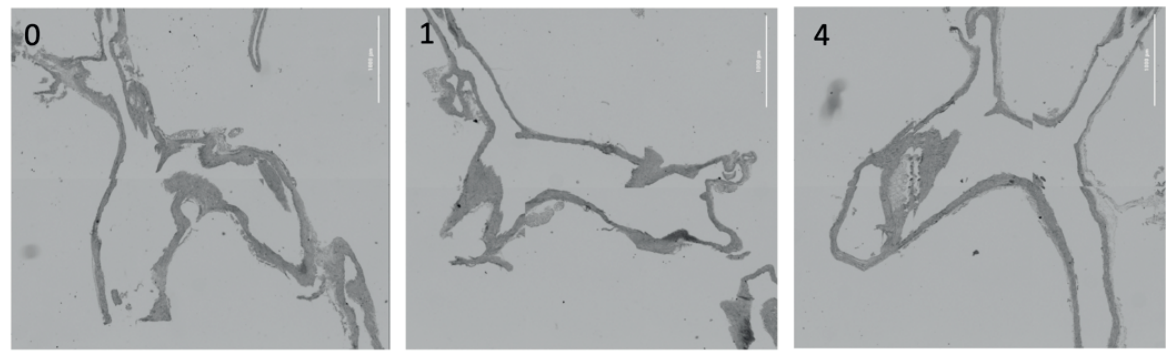

Supplemental figure 1. Hematoxylin and Eosin (HE) staining. Representative images of aortic arches stained for HE. No plaque development was found after VKA treatment.

A

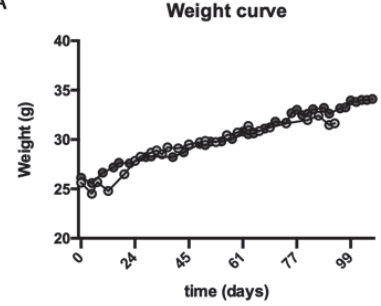

B

$$
\begin{aligned}
& -0-4 \text { wk Warf } \\
& \text {-a } 1 \text { wk Warf }
\end{aligned}
$$

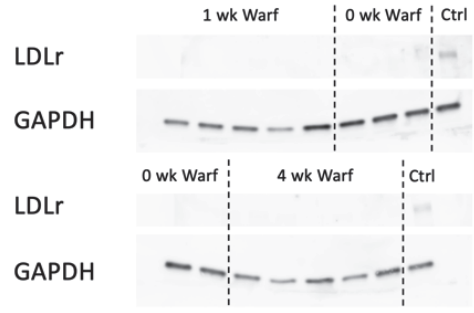

Supplemental figure 2. Weight curve and liver LDL receptor knockdown. (A) Weight curve of animals receiving PCSK9 virus injection and a WTd for 12 weeks. (B) Liver LDL receptor protein expression. All animals that received the PCSK9 overexpressing virus were showing knockdown of the LDL receptor. 


\section{Chapter 4}

Medial vascular smooth muscle cell apoptosis accelerates atherogenesis and promotes features of a vulnerable

plaque

A.M.G. Jaminon, B.A.G. Willems, M. van Zandvoort, C. Recarti, P.M.H. Schiffers, K. Reesink, E.A. Biessen, M. Clarke, M. Bennett, J. de Mey, C. Vermeer, R. Kramann, C.P.M. Reutelingsperger, L.J. Schurgers

In preparation 
Chapter 4

\begin{abstract}
Background: Vascular smooth muscle cells (VSMCs) are involved in many arterial diseases including atherosclerosis and aneurysm formation. In the vessel wall, VSMCs are key players in vascular remodeling, calcification and apoptosis. In a mouse model of inducible VSMC-specific apoptosis, chronic apoptosis of VSMCs in established plaques has been shown to induce features of plaque instability and accelerated atherogenesis. Based on these findings, we studied the role of medial VSMCs on the initiation and progression of atherosclerotic lesions.

Methods: SM22 $\alpha$-iDTR/ApoE ${ }^{-/-}$mice were injected with diphtheria toxin (DT) for 3 weeks to induce medial VSMC apoptosis. After 3 weeks, DT treatment was arrested and some mice were sacrificed $(\mathrm{t}=0)$. Remaining mice were fed a Western type diet (WTD, $0.25 \%$ cholesterol) and sacrificed after 6 and 18 weeks of WTD, respectively. Before sacrifice mice underwent applanation tonometry to measure vessel stiffness. Next, the aortic arch (including branches) was excised and tissues were stained for Hematoxylin \& Eosin, Mac3, Von Kossa, picro Sirius Red and $\alpha$-smooth muscle actin. Additionally, vessel contractility was determined using a wire myograph.

Results: Some $50 \%$ decrease in cellularity was observed in SM22 $\alpha$ $\mathrm{iDTR}+/ \mathrm{ApoE}^{-/-}$mice after DT exposure as compared to control. Vessel wall relaxation was deteriorated in SM22 $\alpha$-iDTR+/ApoE ${ }^{-/-}$mice which was endothelial cell dependent. Additionally, in vitro isolated SM22 $\alpha$-iDTR $+/ \mathrm{ApoE}^{-/}$VSMCs displayed hypertrophy and increased expression of contractile markers. Atherosclerotic plaque size in SM22 $\alpha-$ $\mathrm{iDTR}+/ \mathrm{ApoE}^{-/-}$mice was significantly increased as compared to control mice. After respectively 6 and 18 weeks of WTD, atherosclerotic plaques in SM22 $\alpha$-iDTR+/ApoE ${ }^{-/}$ mice displayed features of plaque vulnerability.

Conclusion: We show that VSMCs are key in maintaining vessel wall homeostasis and that disruption by DT induces vessel wall remodeling, resulting in declined vessel wall responsiveness. Additionally, VSMCs are pivotal in vessel health as depletion results in accelerated atherosclerosis induction and promotes features of a vulnerable plaque.
\end{abstract}




\section{Introduction}

Cardiovascular disease (CVD) is the number one cause of death in the western world and manifests in various phenotypes including atherosclerosis, stroke, myocardial infarction and aneurysm formation 1,2. Despite the development and application of effective treatment, such as the use of medicines and surgery, there is still a relatively high CVD morbidity and mortality ${ }^{3,4}$.

Under physiological conditions, vascular smooth muscle cells (VSMCs) in the tunica media regulate vascular tone and diameter in order to maintain hemodynamic balance ${ }^{5,6}$. Besides VSMCs, extracellular matrix (ECM) proteins including elastin, collagen, fibrinogen and endothelial cells (ECs) are involved in maintenance of physiological vascular function. Remodeling of the vascular media is caused by various stress factors which affect the functionality of the vessel wall ${ }^{6,7}$. Under physiological conditions, the majority of VSMCs in the vessel wall display a contractile phenotype that supports vascular tone. However, a subset of medial VSMCs has the ability to differentiate into a synthetic phenotype ${ }^{8}$. This phenotypic plasticity of VSMCs is necessary to deal with varying conditions of the vascular tissue. Synthetic VSMCs are prone to undergo apoptosis or further phenotypic differentiation towards an osteochondrogenic phenotype ${ }^{9}$. Both apoptosis and osteochondrogenic differentiation of VSMCs are known to contribute to vascular remodeling, which results in adaptation of the vessel wall. Vascular remodeling is related to pathological processes such as inflammation, fibrosis and calcification of arteries. Vascular calcification can lead to increased elastin breaks and further induction of apoptosis of VSMCs ${ }^{10}$. Vascular remodeling has been linked to the development of atherosclerosis and vessel wall occlusion ${ }^{11-13}$. Atherosclerosis is known as a chronic inflammatory disease characterized by endothelial activation and lipid accumulation in the vessel wall ${ }^{14}$. Accumulation of VSMC into the neointima has long been considered a key event in atherosclerosis progression. Here, VSMC promote atherosclerotic plaque stability by depositing extracellular matrix proteins such as collagen to create a thick fibrous cap.

To investigate the role of arterial remodeling on atherosclerosis and features of plaque vulnerability we used SM22 $\alpha-\mathrm{iDTR}+/ \mathrm{ApoE}^{-/-}$mice that are previously described by Clarke and coworkers ${ }^{15,16}$. The authors showed that apoptosis of VSMCs in the vasculature is a key factor in the development of plaque instability in the early and late phase of atherosclerosis ${ }^{15}$. Medial vascular calcification and apoptosis has been shown to affect atherosclerosis formation ${ }^{16-18}$.

In our study, we used SM22 $\alpha-\mathrm{iDTR}+/ \mathrm{ApoE}^{-/-}$mice to investigate the role of VSMCs in the vascular media on vessel wall properties and the initiation and progression of atherosclerosis. VSMC apoptosis, and consequently depletion of medial VSMCs was induced prior to formation of atherosclerotic plaques. We demonstrate the important contribution of medial VSMCs on vessel wall homeostasis and on atherogenesis and plaque stability.

\section{Methods}

\section{Animal protocols}

SM22 $\alpha$-iDTR/ApoE ${ }^{-/-}$mice were kindly provided by Prof. Dr. M. Bennett ${ }^{15}$. Control animals were diphtheria toxin receptor negative littermates (SM22 $\alpha$ $\mathrm{iDTR} / \mathrm{ApoE}^{-/-}$mice $)$. Mice were kept in a temperature-controlled environment $\left(20^{\circ} \mathrm{C}\right)$ 
with a regular day \& night cycle. All animal experiments were conducted under a protocol approved by the local ethics committee for animal experiments, Maastricht University, The Netherlands.

Purified, recombinant, unnicked human diphtheria toxin (DT) (Quadratech Diagnostics, Surrey, United Kingdom) was dissolved in $0.9 \%$ saline solution containing $0.2 \%$ low endotoxin bovine serum albumin (Sigma, Zwijndrecht, the Netherlands), sterile filtered and stored at $-80^{\circ} \mathrm{C}$ until use.

Experimental animals ( 12 week old males) were weighed and injected 3 times a week intraperitoneally with $1 \mathrm{ng} / \mathrm{g}$ bodyweight of DT for 3 weeks. Next, a 1-week wash-out period was provided. During this period, mice were fed a normal chow diet and water ad libitum.

At sacrifice, blood was collected in $3.2 \%$ sodium citrate by vena cava puncture and the aorta was flushed with $100 \mu \mathrm{M}$ sodium nitroprusside in phosphate buffered saline (PBS). Bone marrow was collected by flushing the femur with PBS. The aortic arch and its main branches were excised and fixated in $1 \%$ paraformaldehyde overnight and embedded in paraffin. First-order mesenteric arteries and carotid artery were used in myograph and two-photon imaging experiments. Besides, aortas were snap-frozen in liquid nitrogen and stored in $-80^{\circ} \mathrm{C}$ after which RNA extraction was performed.

To study atherosclerosis development, mice were fed a WTD containing $0.25 \%$ cholesterol, $15 \%$ cacao butter and $1 \%$ corn oil (AB diets, Woerden, the Netherlands) for either 6 or 18 weeks and were sacrificed using the same procedure.

\section{Plasma cholesterol and triglyceride determination}

Total cholesterol (Horiba Medical, Montpellier, France) and triglyceride (Horiba Medical, Montpellier, France) measurements were performed as prescribed.

\section{FACS analysis of blood and bone marrow}

The whole blood cell composition was measured using a haematology-analyzer (XP-300, Sysmex, Etten-Leur, the Netherlands). After measurement, erythrocyte lysis was performed and the blood cell composition was further determined using antibodies against CD11b, CD4, Ly6G, B220 (all BD Biosciences, Temse, the Netherlands), Ly6C (Miltenyi Biotec, Leiden, the Netherlands), CD3e, NK1.1 and CD8a (all eBioscience, Vienna, Austria). Bone marrow cell composition was analysed using antibodies versus Ly6G, Ly6C, B220, NK1.1, CD3e, CD11b and CD115 (eBioscience, Vienna, Austria). Flow cytometry was performed using a FACS Canto (BD Biosciences, Temse, the Netherlands) and analysed using FACSDiva software (v6.1.3, BD Biosciences, Temse, the Netherlands).

\section{Wire Myograph}

Drugs used: Phenylephrine (PHE), Acethylcholine (ACh), Indomethacin (INDO, cyclooxygenase-inhibitor), Sodium Nitroprusside (SNP) and L-NAME (NO synthase inhibitor) were all purchased from Sigma Aldrich (Zwijndrecht, the Netherlands).

First order mesenteric resistance arterial segments of approximately $2 \mathrm{~mm}$ in length were isolated and mounted in wire myographs (Model 610M, DMT, Denmark) for recording of isometric force development. After mounting, the vessel segments were equilibrated for 15 minutes at $37^{\circ} \mathrm{C}$ in an oxygenated $\left(95 \% \mathrm{O}_{2}\right.$ and $\left.5 \% \mathrm{CO}_{2}, \mathrm{pH} 7.4\right)$ 
Krebs-Ringer bicarbonate buffer containing (in $\mathrm{mM}$ ): $118.5 \mathrm{NaCl}, 4.7 \mathrm{KCl}, 2.5 \mathrm{CaCl}_{2}$, $1.2 \mathrm{MgSO}_{4}, 1.2 \mathrm{KH}_{2} \mathrm{PO}_{4}, 25 \mathrm{NaHCO}_{3}$ and 5.5 glucose. Following equilibration, the vessel segments were distended to a diameter corresponding to $90 \%$ of the passive diameter at a transmural pressure of $100 \mathrm{mmHg}{ }^{19}$.

Contractile responses to $\mathrm{KCl}\left(62.5 \mathrm{mM}, \mathrm{K}^{+}\right)$and increasing concentrations of Phenylephrine $(0.01-30 \mu \mathrm{M}, \mathrm{PHE})$ and relaxing responses to increasing concentrations to Acethylcholine (0.01- $10 \mu \mathrm{M}$, ACh) were recorded. The effects of nitric oxide synthase inhibition $(100 \mu \mathrm{M}, \mathrm{L}-\mathrm{NAME})$ and cyclo-oxygenase inhibition $(1 \mu \mathrm{M}$, INDO) were evaluated. Endothelium-independent NO-mediated vasorelaxation was measured using increasing concentrations of Sodium Nitroprusside $(0.001-1 \mu \mathrm{M}, \mathrm{SNP})$ during precontraction with PHE $(30 \mu \mathrm{M})$.

\section{Two-photon imaging}

For imaging of the mounted and pressurized blood vessels, a Leica TCS SP5 (Leica Microsystems GmbH, Wetzlar, Germany) two-photon laser scanning microscope was used with a Ti-Sapphire Chameleon Ultra II (Coherent Inc, Santa Clara, CA, USA) laser. Excitation was at $840 \mathrm{~nm}$ using a fs-pulsed laser (maximal power at the sample at $100 \%$ of $50 \mathrm{~mW}$ ). A Leica objective, HCX APO L 20x/1.00 was used. Fluorescence detection was performed using four detectors (1 forward and 3 backward), set according to the emission spectra. For DAPI a setting between 440 and $500 \mathrm{~nm}$ (channel 1), for the autofluorescence of Elastin a setting between 500-560 nm (channel 2) was used. For the SHG, both in forward (channel 4) and backward (channel 3) direction, a setting of 400440 was used. With this choice of settings DAPI appears strongest in channel 1 and 2 and is color-coded Cyan. Elastin appears in all channels, but strongest in channel 2 and is color-coded green. Backward SHG appears exclusively channel 3 and is color-coded Magenta. Forward SHG is color-coded yellow.

Maximum projection images were made via the Imaris software (Oxford instruments, Bitplane, Zürich, Switserland), looking specifically at channel 2 and separating nuclei from elastin structures using segmentation based on shape. VSMCs were separated from other cell nuclei (fibroblasts and endothelial cells) segmenting on their typical orientation and shape. The segmentation was checked manually and the separated VSMC nuclei were counted in Imaris.

\section{Histology and immunohistochemistry}

$5 \mu \mathrm{M}$ serial sections were cut from paraffin embedded aortic arches and stained with haematoxylin and eosin (H\&E). Plaque burden was analysed on these stained sections using ImageJ software (v1.47). Total plaque burden was measured in the aortic arch and major branching points.

Antigen retrieval was performed by heating samples in target retrieval solution (Dako, Heverlee, Belgium). Subsequently, tissues were blocked and incubated with primary antibodies overnight as described: $\alpha$ SMA (1A4, Dako; 1:500); Mac3 (M3/84; Pharmingen, 1:200). After overnight incubation, sections were incubated for 1 hour with HRP-conjugated secondary antibodies and detected with NovaRED (Vector Labs, Amsterdam, the Netherlands). Apoptotic cells were detected using a terminal deoxynuceotidyl transferase dUTP nick-end labelling (TUNEL) kit (Roche, Woerden, the Netherlands) as described in the manufacturers protocol. Calcification was detected using Von Kossa and collagen fibers using picro sirius red. Additionally, picro sirius red 
stained sections were also used for determination of the necrotic core size (percentage of the plaque) and the thickness of the fibrous cap. Fibrous caps were determined as the collagen rich area overlying the necrotic core. Images were captured using a DM400 microscope (Leica, Eindhoven, the Netherlands) and imaging software (LAS V, Eindhoven, the Netherlands).

\section{RNA extraction, cDNA synthesis and quantative analysis}

Aortic arches we homogenised by glass beads (BioSpec, Bartlesville, USA) in TRI reagent (Sigma). Isolated primary vascular smooth muscle cells (VSCMs) were directly lysed in TRI reagent. After incubation, total RNA was extracted using a phenolchloroform method as previously described by Chomczynski et al ${ }^{20}$. RNA concentration was quantified spectrophotometrically at $260 \mathrm{~nm} .1 \mu \mathrm{g}$ of total RNA was treated with DNase I (Promega) and reverse transcribed using Moloney Murine Leukemia Virus Reverse Transcriptase (M-MLV RT), RNAout, dNTPs, dithiothreitol (all Invitrogen) and an oligo(dT)adapter (Eurogentec) for 1 hour at $37^{\circ} \mathrm{C}$. Gene expression levels were quantified by real-time quantitative PCR (qPCR) on a LightCycler 480 Real-Time PCR instrument (Roche Applied Science). Amplification reactions were carried out in a volume of $10 \mu \mathrm{l}$ including $20 \mathrm{ng}$ of total cDNA, $5 \mu \mathrm{l}$ QuantiTect SYBR Green PCR Kit (Qiagen) and $2 \mu \mathrm{M}$ of each primer (supplemental table 3). An initial denaturation step (15 minutes at $95^{\circ} \mathrm{C}$ ) was followed by 50 cycles of amplification (denaturation: 15 seconds at $95^{\circ} \mathrm{C}$, annealing: 30 seconds at $57^{\circ} \mathrm{C}$ : extension: 45 seconds at $72^{\circ} \mathrm{C}$ ). Specificity was analysed by melting curve plots. Fluorescence curves were analysed with LightCycler 480 Software (Version 1.5) and relative quantification by plotting N0 ration.

\section{Statistical analysis}

All values are shown as mean \pm SEM unless stated differently. Statistical analysis was performed by 1- way ANOVA with Bonferroni post hoc test using PRISM software (GraphPad). Values of $\mathrm{P}<0.05$ were considered statistically significant.

\section{Results}

\section{Diphtheria toxin reduces medial VSMC cellularity in SM22 $\alpha$-iDTR+/ApoE ${ }^{-/-}$mice}

A schematic overview of the animal study performed is displayed in figure $1 \mathrm{~A}$. Mice were injected 3 times/ week for 3 weeks with diphtheria toxin after which a washout period of one week was maintained. At $t=0$, cellularity of the vessel wall was measured for different vessels (Figure 1B). Aorta nuclei were counted by HE stain and SM22 $\alpha$-iDTR+/ApoE ${ }^{-/}$showed some $50 \%$ reduction in cell count compared to SM22 $\alpha-$ iDTR-/ApoE ${ }^{-/}$mice (Figure 1C). First-order mesenteric and carotid artery were imaged by two-photon microscopy and SM $22 \alpha-\mathrm{iDTR}+/ \mathrm{ApoE}^{-/-}$showed some $40 \%$ reduction in the number of cells compared to SM22 $\alpha$-iDTR-/ApoE ${ }^{-/-}$mice (Figures 1 D-F). VSMCs were stained with DAPI to visualize their nuclei (representative images displayed in Figure $1 \mathrm{G}$ and $\mathrm{H}$ ). Using two-photon microscope, 3D reconstruction of the vessel was performed (Figure 1I) and cell number was measured in 3D reconstructed vessel.

Mice fed standard chow diet did not present atherosclerotic plaques in the aortic arch and its main branches of either SM22 $\alpha-\mathrm{hDTr}^{+} / \mathrm{ApoE}^{-/-}$or SM22 $\alpha-\mathrm{hDTr} / \mathrm{ApoE}^{-/-}$ mice at $\mathrm{t}=0$, which is consistent with previously reported data ${ }^{21,22}$. 


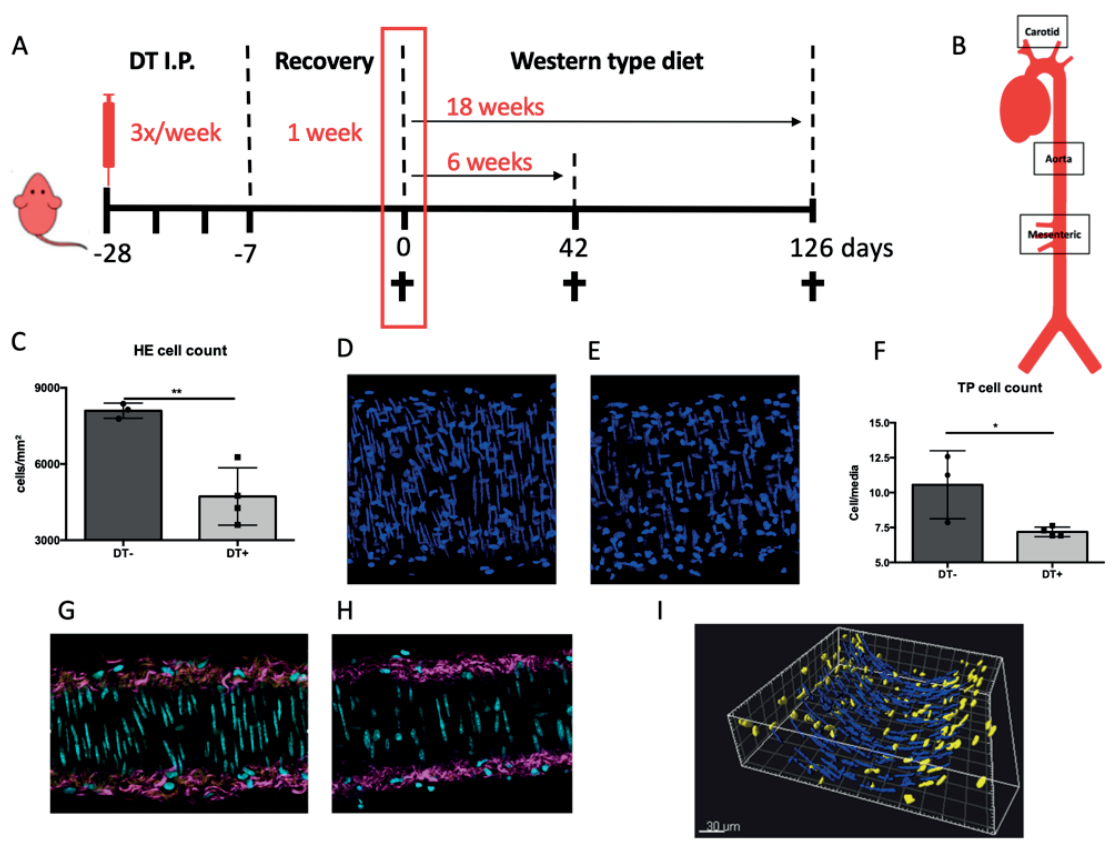

Figure 1. Characterization of mice exposed to DT. (A) Experimental flow chart and SM22 $\alpha-i D T R+/ A_{p o E}$ - model validation at $\mathrm{t}=0$. (B) Schematic representation of the vessels that were used. (C) Cellularity of the vessel wall (cells $/ \mathrm{mm}^{2}$ ) was determined by quantifying the number of nuclei in the vessel wall after the wash out period. SM22 $\alpha$-iDTR+/ApoE $\mathrm{E}^{-/}$mice showed a $50 \%$ reduction in cell count. (D) Representation of an aortic arch of SM22 $\alpha$-iDTR+/ApoE ${ }^{-/-}$mouse. (E) Representation of an aortic arch of SM22 $\alpha-i D T R-/ A p o E^{-/-}$mouse. (F) Cellularity of the vessel wall determined by TP imaging. SM $22 \alpha-\mathrm{iDTR}+/ \mathrm{ApoE}^{-/-}$mice showed a $40 \%$ reduction in cell count. ( $\mathbf{G}$ and $\mathbf{H})$ Representing images of mesenteric artery by TP microscopy of both SM22 $\alpha$ $\mathrm{iDTR}+\mathrm{ApoE}^{-/-}(\mathrm{G})$ and SM22 $\alpha-\mathrm{iDTR}-/ \mathrm{ApoE}^{-/-}(\mathrm{H})$ mice. (I) 3D reconstruction of the vessel wall. ${ }^{*} \mathrm{p}<0.05$; $* * \mathrm{p}<0.001$

Media thickness is increased in SM22 $\alpha$-iDTR+/ApoE $\mathrm{E}^{-/-}$mice and in vitro isolated iDTR+ VSMCs show increased cell size and signs of a contractile phenotype

VSMCs isolated from SM22 $\alpha$-iDTR+/ApoE ${ }^{-/-}$mice aorta's showed hypertrophy when comparing to VSMCs isolated from SM22 $\alpha$-iDTR-/ApoE ${ }^{-/-}$mice (Figures $2 \mathrm{~A}, 2 \mathrm{G}$ ). Also, medial layer thickness of mesenteric arteries in SM22 $\alpha-\mathrm{iDTR}+/ \mathrm{ApoE}^{-/-}$was significantly increased compared to SM22 $\alpha$-iDTR-/ApoE ${ }^{-/}$mice (Figures 2B, 2H). Additionally, mRNA expression of $\alpha \mathrm{SMA}$ and $\mathrm{CNN}-1$ was increased in thoracic aorta and VSMCs isolated from SM22 $\alpha-\mathrm{iDTR}+/ \mathrm{ApoE}^{-/}$(Figures $2 \mathrm{C}-\mathrm{F}$ ). 
A

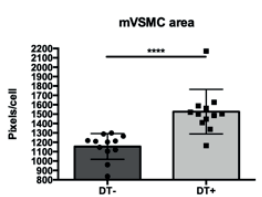

C

VSMC
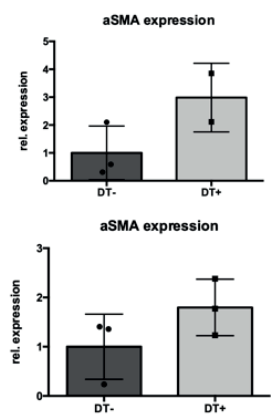

B
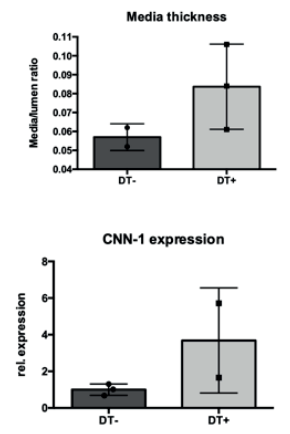

$\mathrm{F}$

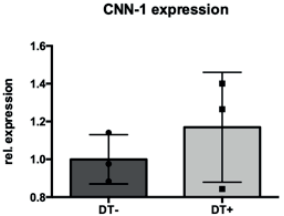

G
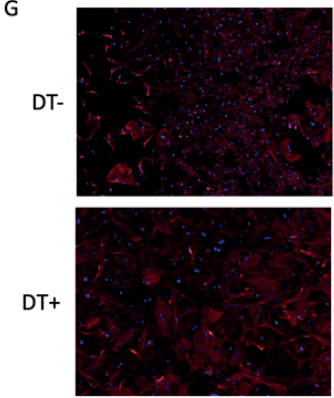

$\mathrm{H}$

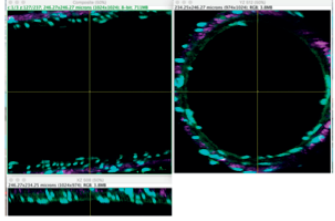

Figure 2. In vitro isolated VSMCs and media thickness. (A) Mouse VSMC cellular size. A significant increase in VSMC size was found in SM22 $\alpha$-iDTR+/ApoE $E^{-/-}$mice. (B) Media thickness determined by TP imaging. (C) Mouse VSMC $\alpha$ SMA RNA expression. (D) Mouse VSMC CNN-1 expression. (E) Thoracic aorta aSMA RNA expression. (F) Thoracic aorta CNN-1 RNA expression. (G) Representative images mVSMC size. Top panel; VSMCs isolated from SM22 $\alpha$-iDTR+/ApoE ${ }^{-/-}$mice, bottom panel; VSMCs isolated from SM22 $\alpha$-iDTR-/ApoE ${ }^{-/ 2}$ mice. (H) TP imaging representation to determine media thickness. ${ }^{* * * *} \mathrm{p}<0.0001$

\section{SM22 $\alpha$-iDTR+/ApoE ${ }^{-/-}$mice show decreased mesenteric artery vessel vasodilation with increased cfPWV}

$\mathrm{SM} 22 \alpha-\mathrm{iDTR}+/ \mathrm{ApoE}^{-/-}$first-order mesenteric arteries (Figure 3A) displayed no change in $\mathrm{K}^{+}$and phenylephrine (PHE) induced contraction (Figure 3B). To assess endothelium-dependent responses, mesenteric arteries were contracted using PHE and next exposed to increasing concentrations of acetylcholine (ACh). SM22 $\alpha$ iDTR+/ApoE ${ }^{-/}$showed a significant decrease in $E_{\max }$ compared to SM22 $\alpha$-iDTR-/ApoE ${ }^{-}$ / mice, while $\mathrm{pEC}_{50}$ remained the same (Figure $3 \mathrm{C}$ ). To assess the contribution of endothelial derived hyperpolarizing factors (EDHF) to ACh induced relaxing responses, arteries were contracted with PHE in the presence of l-NAME (to prevent involvement of NO). Sensitivity and maximal response did not differ significantly between SM22 $\alpha$ $\mathrm{iDTR}+/ \mathrm{ApoE}^{-/-}$and SM22 $\alpha$-iDTR-/ApoE ${ }^{-/-}$mice. However, SM22 $\alpha$-iDTR+/ApoE ${ }^{-/-}$ showed a complete ablation of EDHF-related vessel dilation (Figure 3C). To assess maximal vasodilation, Na-nitroprusside (NP) was added and there was no difference in $\mathrm{pEC}_{50}$ and $\mathrm{E}_{\max }$ between SM22 $\alpha-\mathrm{iDTR}+/ \mathrm{ApoE}^{-/-}$and SM22 $\alpha-\mathrm{iDTR}-/ \mathrm{Apo} \mathrm{E}^{-/-}$mice (Figure 3D). Carotid-femoral pulse wave velocity (cfPWV) was significantly increased in SM22 $\alpha$-iDTR-/ApoE ${ }^{-/-}$compared to SM22 $\alpha$-iDTR+/ApoE ${ }^{-/-}$mice. 
A

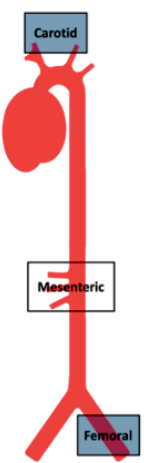

B

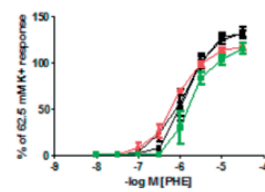

D

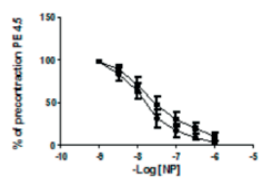

C

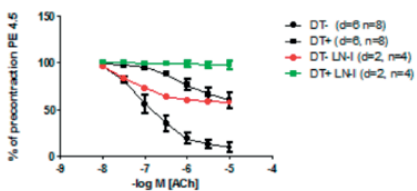

$\mathrm{E}$

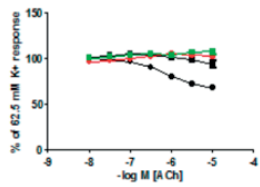

F

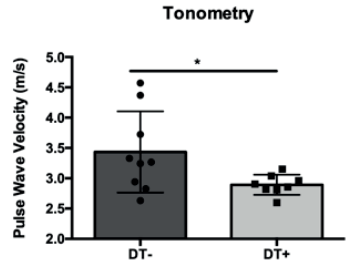

Figure 3. Vessel contractility properties. (A) Schematic representation of the aorta indicating which vessels were used. Blue boxed vessels used in cfPWV, mesenteric arteries (white box) used for myograph measurements. (B) Contractile response to PHE in SM22 $\alpha$-iDTR+/ApoE ${ }^{-/ 2}$ (closed squares) and SM22 $\alpha$-iDTR$/ \mathrm{ApoE}^{-/-}$(closed circles) vessels. (C) Relaxing responses to ACh in SM22 $\alpha-\mathrm{iDTR}+/ \mathrm{ApoE}^{-/-}$(closed squares) and SM22 $\alpha$-iDTR-/ApoE ${ }^{-/-}$(closed circles) vessels, in addition with l-NAME in SM22 $\alpha$-iDTR+/ApoE ${ }^{-/}$(green squares) and SM22 $\alpha-\mathrm{iDTR}-/ \mathrm{ApoE}^{-/-}$(red circles). (D) Relaxing responses to NP in SM22 $\alpha-\mathrm{iDTR}+/ \mathrm{ApoE}^{-/-}$ (closed squares) and SM22 $\alpha$-iDTR-/ApoE ${ }^{-/}$(closed circles) vessels. (E) Relaxing responses to ACh in SM22 $\alpha-$ iDTR+/ApoE ${ }^{-/}$(closed squares) and SM22 $\alpha$-iDTR-/ApoE ${ }^{-/}$(closed circles) vessels, in addition with l-NAME in $\mathrm{SM} 22 \alpha-\mathrm{iDTR}+/ \mathrm{ApoE}^{-/}$(green squares) and SM22 $\alpha-\mathrm{iDTR}_{-} / \mathrm{ApoE}^{-/}$(red circles). (F) cfPWV results. A significant decrease in PWV was found in SM22 $\alpha-\mathrm{iDTR}+/ \mathrm{ApoE}^{-/ /}$mice. ${ }^{*} \mathrm{p}<0.05$

\section{Baseline characteristics of SM22 $\alpha$-iDTR+/-/ApoE ${ }^{-/-}$mice after atherosclerosis induction}

We investigated parameters affecting atherogenesis in both SM22 $\alpha$ iDTR+/ApoE ${ }^{-/-}$and SM22 $\alpha-\mathrm{iDTR}-/ \mathrm{ApoE}^{-/-}$mice. At time of sacrifice, the observed bodyweights after 6 or 18 weeks of WTD did not significantly differ between SM22 $\alpha$ iDTR+/ApoE ${ }^{-/-}$and SM22 $\alpha$-iDTR-/ApoE ${ }^{-/-}$(Table 1). Additionally, blood lipid levels were measured in mice sacrificed after 6 and 18 weeks of WTD. Similarly, there were no significant differences in cholesterol and triglyceride levels between SM22 $\alpha$ iDTR+/ApoE ${ }^{-/-}$and SM22 $\alpha-\mathrm{iDTR}_{-} / \mathrm{ApoE}^{-/-}$mice (Table 1). At the time of sacrifice, no organ abnormalities were observed in mice of both strains, as has been shown before ${ }^{15}$.

Following treatment with DT, circulating leukocyte profiles had not significantly altered, both in SM22 $\alpha$-iDTR+/ApoE ${ }^{-/-}$and SM22 $\alpha$-iDTR-/ApoE ${ }^{-/-}$mice (Supplemental table 1). Analysis of the leukocyte populations in bone marrow did reveal a difference. After 6 weeks of WTD, B-cell levels had significantly increased in SM22 $\alpha$ iDTR+/ApoE ${ }^{-/-}$mice as compared to SM22 $\alpha$-iDTR-/ApoE ${ }^{-/-}$mice (Supplemental Table 
2). No significant differences were observed between other leukocyte populations in bone marrow from SM22 $\alpha-\mathrm{iDTR}+/ \mathrm{ApoE}^{-/}$and SM22 $\alpha-\mathrm{iDTR}-/ \mathrm{ApoE}^{-/-}$mice.

Table 1. Baseline characteristics of SM22 $\alpha$-iDTR-/ApoE ${ }^{-/-}$and SM22 $\alpha-$ iDTR+/ApoE $E^{-/}$mice. All values are represented as mean \pm SD. Significance was analyzed using a one-way ANOVA with Bonferroni's multiple comparison test.

\begin{tabular}{|c|c|c|c|c|}
\hline & $\begin{array}{c}6 \text { weeks } \\
\text { SM22a-hDTr/ApoE } / \%\end{array}$ & 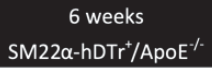 & $\begin{array}{c}18 \text { weeks } \\
\text { SM22a-hDTr/ApoE }{ }^{-1}\end{array}$ & 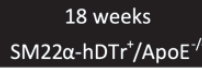 \\
\hline Weight (g) & $29,00 \pm 1,30$ & $28,40 \pm 1,80$ & $33,20 \pm 3,60$ & $32,2 \pm 3,60$ \\
\hline Cholesterol (mM) & $5,77 \pm 0,70$ & $6,21 \pm 1,24$ & $5,21 \pm 0,80$ & $5,66 \pm 0,59$ \\
\hline Triglycerides $(\mathrm{mM})$ & $1,49 \pm 0,30$ & $1,67 \pm 0,94$ & $1,47 \pm 0,53$ & $1,62 \pm 0,58$ \\
\hline
\end{tabular}

\section{DT induced medial VSMC apoptosis accelerates early and late atherogenesis}

After DT induction and VSMC ablation mice were fed a WTD for either 6 or 18 weeks (Figure 4A). After 6 weeks of WTD, atherosclerotic plaques were present in both SM22 $\alpha-\mathrm{iDTR}+/ \mathrm{ApoE}^{-/-}$and SM22 $\alpha-\mathrm{iDTR}-/ \mathrm{ApoE}^{-/-}$mice (Figures 4B and C). Quantification of plaque size using H\&E stained aortic sections showed a 2-fold larger atherosclerotic plaque burden in SM22 $\alpha$-iDTR $+/ \mathrm{ApoE}^{-/}$mice as compared to SM22 $\alpha$ iDTR/ApoE ${ }^{-/}$littermates. After 18 weeks of WTD (Figures 4B and C), a similar 2-fold significantly increased plaque burden was observed in SM22 $\alpha$-iDTR $+/ \mathrm{ApoE}^{-/-}$mice (Figure 4C).

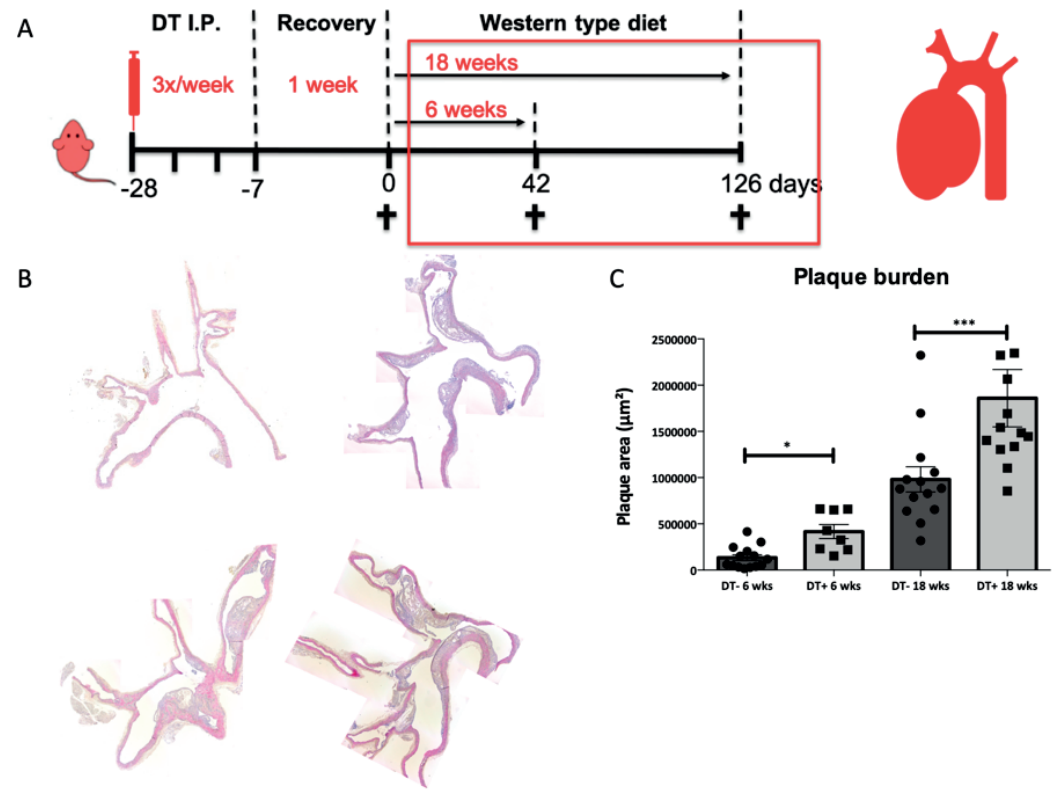

Figure 4. VSMCs apoptosis accelerates atherosclerosis. (A) Schematic overview of the study indicating the period of pro-atherogenic diet. (B and C) After 6 weeks of WTD, atherosclerotic plaques in SM22 $\alpha$ $\mathrm{iDTR}+/ \mathrm{ApoE}^{-/-}$mice are significantly larger as compared to SM22 $\alpha-\mathrm{iDTR}-/ \mathrm{ApoE}^{-/-}$mice. Representative images of the aortic arch and main branch points are shown (panel B, top left: SM22 $\alpha$-iDTR-/ApoE ${ }^{-/}$, top right: SM22 $\alpha$-iDTR+/ApoE ${ }^{-/}$). After 18 weeks of WTD, atherosclerotic plaques in DT mice are significantly larger as compared to WT mice. Representative images of the aortic arch and main branch points are shown (panel B, bottom left: SM22 $\alpha$-iDTR-/ApoE $E^{-/}$, bottom right: SM22 $\alpha-\mathrm{iDTR}+/ \mathrm{ApoE}^{-/}$). (C) Quantification of atherosclerotic plaque area. Images were taken at $4 \mathrm{x}$ magnification. ${ }^{*} \mathrm{p}<0.05 ; * * * \mathrm{p}<0.001$ 


\section{Reduced medial VSMC number is associated with features of plaque vulnerability}

In addition to atherosclerotic plaque burden, atherosclerotic plaques of both $\mathrm{SM} 22 \alpha-\mathrm{iDTR}+/ \mathrm{ApoE}^{-/-}$and SM22 $\alpha-\mathrm{iDTR}-/ \mathrm{ApoE}^{-/-}$mice were analyzed for features of plaque vulnerability. Atherosclerotic plaques of SM22 $\alpha$-iDTR $+/ \mathrm{ApoE}^{-/-}$mice had more Mac3 positive stain after 6 weeks of WTD, indicative of a more inflammatory plaque phenotype, in comparison to SM22 $\alpha$-iDTR-/ApoE-/- mice (Figures 5A and 5B). After 18 weeks of WTD, the difference in Mac3 disappeared.

Next, we analyzed atherosclerotic plaque calcification in both SM22 $\alpha$ iDTR+/ApoE ${ }^{-/}$and SM22 $\alpha-\mathrm{iDTR}_{-} / \mathrm{ApoE}^{-/-}$mice (Figures 6B and 6D). After 6 weeks of WTD, no calcification was detectable in both SM22 $\alpha$-iDTR+/ApoE ${ }^{-/}$or SM22 $\alpha$-iDTR$/ \mathrm{ApoE}^{-/-}$(data not shown). After 18 weeks of WTD, calcification was significantly present. However, there was no significant difference in relative plaque calcification between SM22 $\alpha$-iDTR+/ApoE ${ }^{-/-}$and SM22 $\alpha$-iDTR-/ApoE ${ }^{-/-}$mice (Figure 5C and 5D). However, when calculating absolute values, there is an increase in plaque calcification of $72 \%$ between SM22 $\alpha$-iDTR+/ApoE ${ }^{-/-}$(mean $31123 \mu \mathrm{m}^{2} \pm 28221 \mu \mathrm{m}^{2}$ ) and SM22 $\alpha-$ iDTR-/ApoE ${ }^{-/-}\left(\right.$mean $\left.18676 \mu \mathrm{m}^{2} \pm 14606 \mu \mathrm{m}^{2}\right)$ mice $(\mathrm{p}=0.081$; Figure 5E).

A

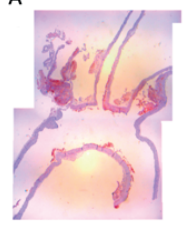

C
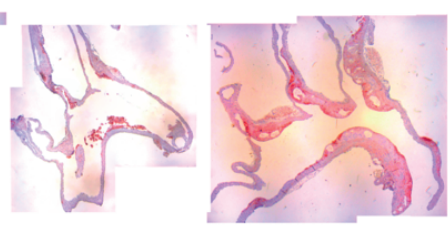

D

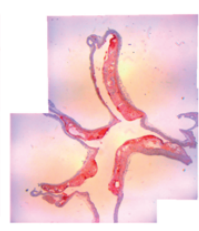

vK positivity

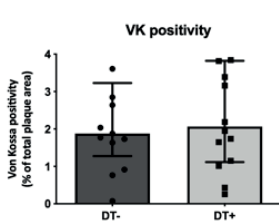

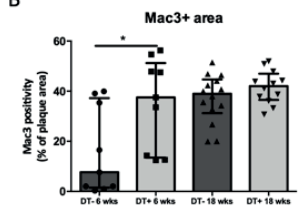

$E$
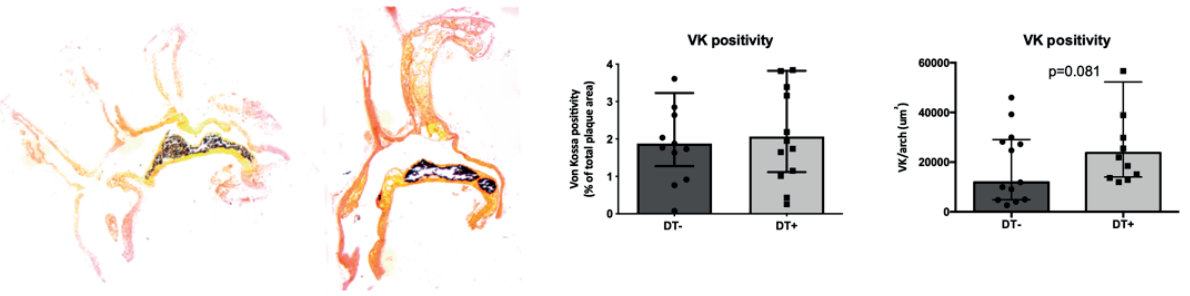

Figure 5. Plaque macrophage infiltration and calcification. (A) Representative images of Mac3+ IHC staining. (B) Quantification of Mac3+ per plaque, in which a significant increase is found in the SM22 $\alpha-$ iDTR+/ApoE ${ }^{-/}$vs. SM22 $\alpha$-iDTR-/ApoE ${ }^{-/}$group at 6 weeks. (C) Representation of Von Kossa staining which was only detected at the later time point of 18 weeks. (D) Quantification of relative Von Kossa positivity in which no differences were found between SM22 $\alpha-\mathrm{iDTR}+/ \mathrm{ApoE}^{-/-}$and SM22 $\alpha-\mathrm{iDTR}-/ \mathrm{ApoE}^{-/}$. (E) Quantification of absolute Von Kossa positivity in which a trend was found between SM22 $\alpha-\mathrm{iDTR}+/ \mathrm{ApoE}^{-/-}$ and SM22 $\alpha$-iDTR-/ApoE ${ }^{-/}$. All images were taken at $4 \mathrm{x}$ magnification. ${ }^{*} \mathrm{p}<0.05$

VSMCs are key to build the atherosclerotic fibrous cap. SM22 $\alpha-i \mathrm{DTR}+/ \mathrm{ApoE}^{-}$ - mice displayed significantly thinner fibrous caps as deduced from picro sirius red staining (Figures 6A and 6B) at both 6 and 18 weeks of WTD. Collagen content in atherosclerotic plaques was not significantly different after both 6 and 18 weeks of WTD (Figure 6C and 6D).

After 6 weeks of WTD, no necrotic core could be observed in either SM22 $\alpha$ iDTR+/ApoE ${ }^{-/}$or SM22 $\alpha$-iDTR-/ApoE ${ }^{-/-}$mice. After 18 weeks SM22 $\alpha-i D T R+/ A_{p o E}{ }^{-/-}$ 
mice had significantly larger necrotic cores as compared to SM22 $\alpha-\mathrm{iDTR}-/$ ApoE $^{-/-}$ littermates (Figures 6E and 6F).

Subsequently, VSMC content of atherosclerotic plaques in both SM22 $\alpha$ iDTR+/ApoE ${ }^{-/-}$and SM22 $\alpha-$ iDTR-/ApoE ${ }^{-/-}$mice after 6 or 18 weeks of western type diet was analyzed. After 6 weeks, significantly less VSMC were present in plaques of $\mathrm{SM} 22 \alpha-\mathrm{iDTR}+/ \mathrm{ApoE}^{-/-}$mice as compared to SM22 $\alpha$-iDTR-/ApoE ${ }^{-/-}$mice (Figures $6 \mathrm{G}$ and $\mathrm{H}$ ). After 18 weeks, the difference in plaque VSMC number disappeared. TUNEL staining revealed no difference in the degree of apoptosis in plaques of either SM22 $\alpha$ iDTR+/ApoE ${ }^{-/-}$or SM22 $\alpha$-iDTR-/ApoE ${ }^{-/-}$mice after 6 or 18 weeks of western type diet (data not shown).
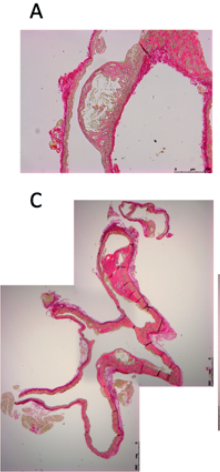
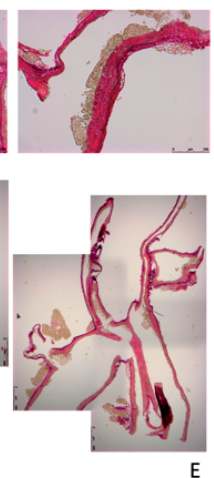
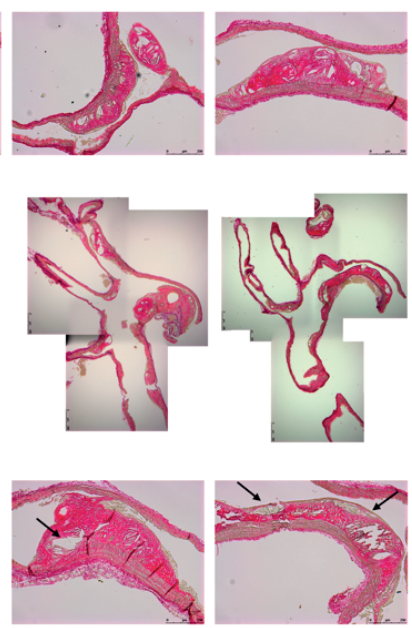
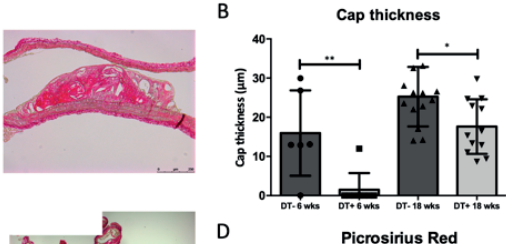

D

Picrosirius Red
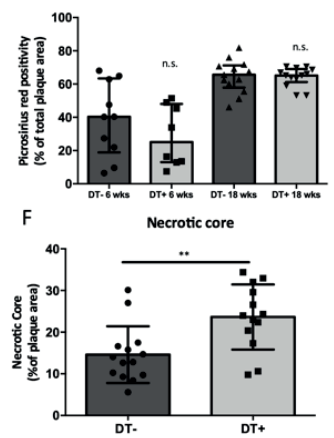

aSMA positivity

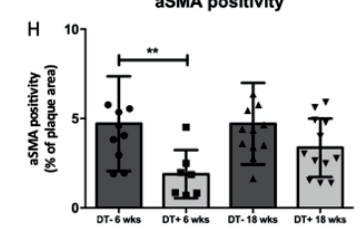

G

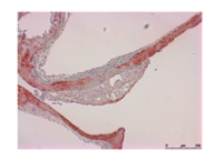

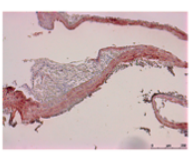

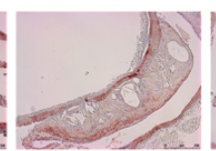

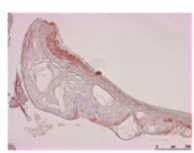

Figure 6. Plaque vulnerability features. (A) Representation of picro Sirius Red staining (PSR) images that were taken to assess cap thickness. (B) At both time points SM22 $\alpha$-iDTR+/ApoE-/- mice were showing thinner fibrous caps compared to SM22 $\alpha$-iDTR-/ApoE-/-. (C) Representation of overall PSR staining. (D) No significant differences were found in SM22 $\alpha$-iDTR+/ApoE-/- vs. SM22 $\alpha$-iDTR-/ApoE-/- mice when looking at collagen content. (E) Representation of PSR images for necrotic core determination. (F) A significant increase of necrotic core was found in SM22 $\alpha$-iDTR+/ApoE-/- plaques compared to SM22 $\alpha$-iDTR-/ApoE-/-. (G) Representative images of aSMA positive stained images. (H) A significant decrease in aSMA positively was found in SM22 $\alpha$-iDTR+/ApoE-/- vs. SM22 $\alpha$-iDTR-/ApoE-/- mice after 6 weeks of diet. (C) Images were taken at $4 \mathrm{x}$ magnification. (A), (E) and (G) Images were taken at $10 \mathrm{x}$ magnification. ${ }^{*} \mathrm{p}<0.05 ; * * \mathrm{p}<0.01$ 


\section{Discussion}

In this paper, we studied the role of medial VSMC apoptosis on atherogenesis. VSMC-specific apoptosis of medial VSMCs was induced in SM22 $\alpha-i D T R+/ A_{p o E}{ }^{-/}$ mice to study the contribution of vascular medial remodeling in atherosclerosis. We demonstrate that a loss of medial VSMCs prior to atherosclerosis results in accelerated de novo and on the long term larger atherosclerotic plaques. Additionally, atherosclerotic plaques had features of a more vulnerable phenotype, characterized by a thin fibrous cap, a large necrotic core, increased inflammation and reduced number of VSMCs.

Our observation that reducing VSMCs in the medial layer prior to atherogenesis accelerates plaque growth, extends the findings of Clarke and coworkers who demonstrated that VSMC apoptosis in mice with established atherosclerotic plaques increased atherosclerotic plaque size and calcification including medial degeneration 15,16. In our mouse model, the accelerated growth of atherosclerotic plaques might be caused by a number of reasons. Firstly, VSMCs slow down plaque growth by formation of a thick fibrous cap, which inhibits monocyte and macrophage infiltration into the atherosclerotic plaque and reduces plaque inflammation and necrotic core formation ${ }^{16}$. Fibrous cap thickness depends on the number of VSMCs in the fibrous cap and the extracellular matrix they deposit ${ }^{23,24}$. Our data support these observations as atherosclerotic plaques of SM22 $\alpha-\mathrm{iDTR}+/ \mathrm{ApoE}^{-/-}$mice show significantly less $\alpha \mathrm{SMA}$ positive cells and a much thinner fibrous cap.

Secondly, VSMCs are efficient efferocytes and contribute to clearance of apoptotic remnants in their environment ${ }^{25}$. Due to their high plasticity, VSMCs can switch to a macrophage-like phenotype expressing typical macrophage markers like CD68 and Mac2 26-28. However, during hyperlipidaemia, defective phagocytosis is induced when VSMCs switch to macrophage-like cells accompanied by IL-1 driven inflammation ${ }^{29,30}$. Apoptotic cell remnants like apoptotic bodies serve as a nidus for vascular calcification ${ }^{10,31}$. Additionally, clearance of apoptotic cells in the atherosclerotic plaque is severely impaired, in part due to oxidative stress and cytoplasmic saturation of indigestible components ${ }^{30}$. Reduced efferocytosis has been demonstrated to promote atherosclerotic plaque growth ${ }^{32,33}$. Hence, the pro-calcifying environment in atherosclerotic plaques, combined with poor clearance of apoptotic bodies form a strong stimulus for calcification in this situation ${ }^{34,35}$. Additionally, we need to reconsider the use of therapies aiming to reduce restenosis by inducing VSMC apoptosis as these therapies might have drastic effects on the stability of developing and established atherosclerotic plaques in patients ${ }^{36-38}$.

In our study, apoptosis of medial VSMC is induced prior to atherogenesis. Under physiological conditions, apoptotic cells are rapidly cleared from the vasculature 39. In contrast to previous results, we did not observe a difference in atherosclerotic plaque calcification. However, absolute values of calcification were increased in SM22 $\alpha-\mathrm{iDTR}_{+} / \mathrm{ApoE}^{-/-}$compared to SM22 $\alpha-\mathrm{iDTR}-/ \mathrm{ApoE}^{-/-}$mice. Clinically, coronary artery calcification (CAC) volume is positively associated with CVD ${ }^{40}$ and higher risk for rupture ${ }^{41}$. Our results support these clinical data that increased of plaque calcification volume results in decreased stability of atherosclerotic plaques ${ }^{42}$.

Aging of the vessel wall is accompanied by vascular remodeling with loss of elasticity and increased deposition of calcium in the media. Moreover, aging is related to medial calcification and thus a higher cardiovascular morbidity and mortality ${ }^{43}$. For 
a long time, it has been demonstrated that calcification and degeneration of the media is an important risk factor for developing atherosclerosis ${ }^{17}$. Clarke and coworkers showed that inducing apoptosis of VSMCs in established atherosclerotic plaques induced intimal calcification. We show that reduced medial VSMC number results in accelerated development of atherosclerosis.

Initially, VSMCs were thought to promote atherosclerotic plaque growth by migrating into the plaque and proliferate, causing plaque growth and occlusion of the vessel $15,16,44$. However, our study demonstrates that medial VSMCs significantly contribute to both inhibition and stability of atherosclerotic plaques. This may occur through several mechanisms, including the interaction between VSMCs and ECs. Here we show that the loss of medial VSMCs is compensated by hypertrophy of the remaining VSMCs. Isolated VSMCs in vitro display signs of increased contractility, which might be a response to maintain vessel wall contraction. Ex vivo myograph analysis revealed significant differences in vessel dilation, with a clear reduced dilation capability in vessels with hypertrophic VSMCs, an effect caused by a loss of endothelial derived hyperpolarizing factors. It is noteworthy that the communication between VSMCs and ECs is affected without disturbing NO mediated pathways. The exact mechanism by which this decrease in vessel wall dilation properties are caused is not fully understood but might arise from the impaired cellular connections in gap junctions between the ECs and the VSMCs ${ }^{45}$. The reduced capacity in interaction between ECs and VSCMs might lead to EC activation with subsequent VSMC phenotype switching, consequently contributing to the development of atherosclerosis ${ }^{46,47}$. This corroborates with our findings of a more pro-inflammatory plaque phenotype in mice that had reduced medial VSMCs. Finally, our data revealed decreased cfPWV in mice with reduced medial VSMCs, indicating that vessel compliance is increased.

In conclusion, we show that medial VSMCs are important for maintaining vessel wall integrity and in preventing vascular medial remodeling and atherosclerosis. Our work highlights the need for further studies to unravel the driving forces of medial remodeling as an inducer of accelerated atherosclerosis. 


\section{References}

1. GBD 2015 Mortality and Causes of Death Collaborators. Global, regional, and national life expectancy, all-cause mortality, and cause-specific mortality for 249 causes of death, 1980-2015: a systematic analysis for the Global Burden of Disease Study 2015. Lancet 388, 1459-1544 (2016).

2. Willems, B. A. G., Vermeer, C., Reutelingsperger, C. P. M. \& Schurgers, L. J. The realm of vitamin K dependent proteins: Shifting from coagulation toward calcification. Mol Nutr Food Res 58, 16201635 (2014).

3. Nichols, M., Townsend, N., Scarborough, P. \& Rayner, M. Cardiovascular disease in Europe 2014: epidemiological update. Eur. Heart J. 35, 2950-2959 (2014).

4. Kandaswamy, E. \& Zuo, L. Recent Advances in Treatment of Coronary Artery Disease: Role of Science and Technology. Int J Mol Sci 19, 424 (2018).

5. Owens, G. K., Kumar, M. S. \& Wamhoff, B. R. Molecular Regulation of Vascular Smooth Muscle Cell Differentiation in Development and Disease. Physiological Reviews 84, 767-801 (2004).

6. Jaminon, A., Reesink, K., Kroon, A. \& Schurgers, L. The Role of Vascular Smooth Muscle Cells in Arterial Remodeling: Focus on Calcification-Related Processes. Int J Mol Sci 20, 5694 (2019).

7. Shanahan, C. M., Crouthamel, M. H., Kapustin, A. \& Giachelli, C. M. Arterial calcification in chronic kidney disease: key roles for calcium and phosphate. Circ. Res. 109, 697-711 (2011).

8. Gerthoffer, W. T. Mechanisms of vascular smooth muscle cell migration. Circ. Res. 100, 607-621 (2007).

9. Chatrou, M. L. L., Winckers, K., Hackeng, T. M., Reutelingsperger, C. P. \& Schurgers, L. J. Vascular calcification: The price to pay for anticoagulation therapy with vitamin $\mathrm{K}$-antagonists. YBLRE 26, 155-166 (2012).

10. Proudfoot, D. et al. Apoptosis regulates human vascular calcification in vitro: evidence for initiation of vascular calcification by apoptotic bodies. Circ. Res. 87, 1055-1062 (2000).

11. Pasterkamp, G. et al. Paradoxical arterial wall shrinkage may contribute to luminal narrowing of human atherosclerotic femoral arteries. Circulation 91, 1444-1449 (1995).

12. Nishioka, T. et al. Contribution of inadequate compensatory enlargement to development of human coronary artery stenosis: an in vivo intravascular ultrasound study. J. Am. Coll. Cardiol. 27, 15711576 (1996).

13. Smits, P. C. et al. Shrinkage of human coronary arteries is an important determinant of de novo atherosclerotic luminal stenosis: an in vivo intravascular ultrasound study. Heart 79, 143-147 (1998).

14. Ross, R. Atherosclerosis--an inflammatory disease. N. Engl. J. Med. 340, 115-126 (1999).

15. Clarke, M. C. H. et al. Apoptosis of vascular smooth muscle cells induces features of plaque vulnerability in atherosclerosis. Nat. Med. 12, 1075-1080 (2006).

16. Clarke, M. C. H. et al. Chronic apoptosis of vascular smooth muscle cells accelerates atherosclerosis and promotes calcification and medial degeneration. Circ. Res. 102, 1529-1538 (2008).

17. Blumenthal, H. T., Lansing, A. I. \& Wheeler, P. A. Calcification of the Media of the Human Aorta and Its Relation to Intimal Arteriosclerosis, Ageing and Disease. The American Journal of Pathology 20, 665-687 (1944).

18. Kothapalli, D. et al. Cardiovascular protection by ApoE and ApoE-HDL linked to suppression of ECM gene expression and arterial stiffening. Cell Rep 2, 1259-1271 (2012).

19. Mulvany, M. J. \& Halpern, W. Contractile properties of small arterial resistance vessels in spontaneously hypertensive and normotensive rats. Circ. Res. 41, 19-26 (1977).

20. Chomczynski, P. \& Sacchi, N. Single-step method of RNA isolation by acid guanidinium thiocyanate-phenol-chloroform extraction. Anal. Biochem. 162, 156-159 (1987).

21. Nakashima, Y., Plump, A. S., Raines, E. W., Breslow, J. L. \& Ross, R. ApoE-deficient mice develop lesions of all phases of atherosclerosis throughout the arterial tree. Arterioscler. Thromb. 14, 133140 (1994).

22. Meir, K. S. \& Leitersdorf, E. Atherosclerosis in the apolipoprotein-E-deficient mouse: a decade of progress. Arteriosclerosis, Thrombosis, and Vascular Biology 24, 1006-1014 (2004).

23. Ross, R. The pathogenesis of atherosclerosis: a perspective for the 1990s. Nature 362, 801-809 (1993).

24. Newby, A. C. \& Zaltsman, A. B. Fibrous cap formation or destruction--the critical importance of vascular smooth muscle cell proliferation, migration and matrix formation. Cardiovasc. Res. 41, 345-360 (1999).

25. Bennett, M. R., Gibson, D. F., Schwartz, S. M. \& Tait, J. F. Binding and phagocytosis of apoptotic vascular smooth muscle cells is mediated in part by exposure of phosphatidylserine. Circ. Res. 77, 1136-1142 (1995). 


\section{Chapter 4}

26.

Feil, S. et al. Transdifferentiation of vascular smooth muscle cells to macrophage-like cells during atherogenesis. Circ. Res. 115, 662-667 (2014).

Rong, J. X., Shapiro, M., Trogan, E. \& Fisher, E. A. Transdifferentiation of mouse aortic smooth muscle cells to a macrophage-like state after cholesterol loading. Proc. Natl. Acad. Sci. U.S.A. 100, 13531-13536 (2003).

Gomez, D. \& Owens, G. K. Smooth muscle cell phenotypic switching in atherosclerosis. Cardiovasc. Res. 95, 156-164 (2012).

Clarke, M. C. H., Talib, S., Figg, N. L. \& Bennett, M. R. Vascular smooth muscle cell apoptosis induces interleukin-1-directed inflammation: effects of hyperlipidemia-mediated inhibition of phagocytosis. Circ. Res. 106, 363-372 (2010).

Schrijvers, D. M., De Meyer, G. R. Y., Kockx, M. M., Herman, A. G. \& Martinet, W. Phagocytosis of apoptotic cells by macrophages is impaired in atherosclerosis. Arteriosclerosis, Thrombosis, and Vascular Biology 25, 1256-1261 (2005).

1. Proudfoot, D. et al. The role of apoptosis in the initiation of vascular calcification. $Z$ Kardiol 90

Suppl 3, 43-46 (2001). atherosclerosis in mice. Circulation 115, 2168-2177 (2007).

Thorp, E., Cui, D., Schrijvers, D. M., Kuriakose, G. \& Tabas, I. Mertk receptor mutation reduces efferocytosis efficiency and promotes apoptotic cell accumulation and plaque necrosis in atherosclerotic lesions of apoe-/- mice. Arteriosclerosis, Thrombosis, and Vascular Biology 28, $1421-1428$ (2008).

Iyemere, V. P., Proudfoot, D., Weissberg, P. L. \& Shanahan, C. M. Vascular smooth muscle cell phenotypic plasticity and the regulation of vascular calcification. J. Intern. Med. 260, 192-210 (2006).

Schurgers, L. J. et al. Vitamin K-antagonists accelerate atherosclerotic calcification and induce a vulnerable plaque phenotype. PLoS ONE 7, e43229 (2012).

Marx, S. O., Jayaraman, T., Go, L. O. \& Marks, A. R. Rapamycin-FKBP inhibits cell cycle regulators of proliferation in vascular smooth muscle cells. Circ. Res. 76, 412-417 (1995).

Marx, S. O., Totary-Jain, H. \& Marks, A. R. Vascular smooth muscle cell proliferation in restenosis. Circ Cardiovasc Interv 4, 104-111 (2011).

Aizawa, Y., Kawabe, J., Hasebe, N., Takehara, N. \& Kikuchi, K. Pioglitazone enhances cytokineinduced apoptosis in vascular smooth muscle cells and reduces intimal hyperplasia. Circulation 104, 455-460 (2001).

Platt, N., da Silva, R. P. \& Gordon, S. Recognizing death: the phagocytosis of apoptotic cells. Trends Cell Biol. 8, 365-372 (1998).

Criqui, M. H. et al. Calcium density of coronary artery plaque and risk of incident cardiovascular events. JAMA 311, 271-278 (2014).

Criqui, M. H. et al. Coronary Artery Calcium Volume and Density: Potential Interactions and Overall Predictive Value: The Multi-Ethnic Study of Atherosclerosis. JACC Cardiovasc Imaging 10, 845854 (2017).

Goldberger, Z. D. et al. Are changes in carotid intima-media thickness related to risk of nonfatal myocardial infarction? A critical review and meta-regression analysis. American Heart Journal 160, 701-714 (2010).

Lanzer, P. et al. Medial vascular calcification revisited: review and perspectives. Eur. Heart J. 35, $1515-1525$ (2014).

Kannel, W. B. \& McGee, D. L. Diabetes and cardiovascular risk factors: the Framingham study. Circulation 59, 8-13 (1979).

Sandow, S. L., Senadheera, S., Bertrand, P. P., Murphy, T. V. \& Tare, M. Myoendothelial contacts, gap junctions, and microdomains: anatomical links to function? Microcirculation 19, 403-415 (2012).

Zhang, Z. et al. Role of Myoendothelial Gap Junctions in the Regulation of Human Coronary Artery Smooth Muscle Cell Differentiation by Laminar Shear Stress. Cell. Physiol. Biochem. 39, 423-437 (2016).

Chae, C.-W. \& Kwon, Y.-W. Cell signaling and biological pathway in cardiovascular diseases. Arch. Pharm. Res. 42, 195-205 (2019). 
Medial VSMC apoptosis accelerates atherogenesis and induces plaque vulnerability

\section{Supplemental material}

Supplemental table 1. Flow cytometric analysis of blood after 6 and 18 weeks of western type diet. All values are represented as mean \pm SD. Significance was analyzed using a one-way ANOVA with Bonferroni's multiple comparison test.

\begin{tabular}{|c|c|c|c|c|c|c|}
\hline $\begin{array}{c}\text { Celltype } \\
\text { (\% of viable } \\
\text { cells) }\end{array}$ & $\begin{array}{l}\text { Subpopulation } \\
\text { ( } \% \text { of parent) }\end{array}$ & $\begin{array}{c}6 \text { weeks } \\
\text { SM22 } \alpha-h D T r \\
/ A p o E^{-}\end{array}$ & $\begin{array}{c}6 \text { weeks } \\
\text { SM22 } \alpha- \\
\text { hDTr }^{+} / \text {ApoE }^{-}\end{array}$ & $\begin{array}{c}18 \text { weeks } \\
\text { SM22 } \alpha-\text { hDTr } \\
\text { /ApoE } \%\end{array}$ & $\begin{array}{c}18 \text { weeks } \\
\text { SM22 } \alpha- \\
\text { hDTr }^{+} / \text {ApoE }^{-/}\end{array}$ & $\mathbf{P}$ \\
\hline B-cells & & $37,8 \pm 11,19$ & $40,13 \pm 21,08$ & $25,31 \pm 13,20$ & $28,59 \pm 9,25$ & n.s \\
\hline \multirow[t]{3}{*}{ T-cells } & & $10,30 \pm 2,25$ & $12,79 \pm 3,40$ & $8,90 \pm 5,07$ & $7,64 \pm 2,34$ & n.s \\
\hline & CD4+ T-cells & $54,56 \pm 2,61$ & $52,31 \pm 2,87$ & $47,85 \pm 4,30$ & $46,25 \pm 4,22$ & n.s \\
\hline & CD8+ T-cells & $51,35 \pm 3,40$ & $43,68 \pm 6,72$ & $55,96 \pm 7,11$ & $52,28 \pm 6,00$ & n.s \\
\hline NK-cells & & $5,62 \pm 1,43$ & $4,10 \pm 1,21$ & $3,55 \pm 1,24$ & $3,15 \pm 0,73$ & n.s \\
\hline Granulocytes & & $65,35+9,12$ & $69,21+15,86$ & $66,64+13,30$ & $67,55+9,241$ & n.s \\
\hline \multirow[t]{4}{*}{ Monocytes } & & $29,28 \pm 7,55$ & $24,95 \pm 13,12$ & $27,49 \pm 11,36$ & $27,93 \pm 7,95$ & n.s \\
\hline & Ly6C low & $28,78 \pm 13,15$ & $22,04 \pm 7,59$ & $22,28 \pm 9,75$ & $17,43 \pm 6,48$ & n.s \\
\hline & Ly6C medium & $6,03 \pm 1,50$ & $6,71 \pm 2,84$ & $3,15 \pm 1,03$ & $3,47 \pm 0,95$ & n.s \\
\hline & Ly6C high & $53,97 \pm 14,33$ & $55,25 \pm 15,78$ & $59,00 \pm 13,35$ & $67,00 \pm 11,41$ & n.s \\
\hline
\end{tabular}

Supplemental table 2. Flow cytometric analysis of bone marrow after 6 and 18 weeks of western type diet. All values are represented as mean \pm SD. Significance was analyzed using a one-way ANOVA with Bonferroni's multiple comparison test.

\begin{tabular}{|c|c|c|c|c|c|c|}
\hline $\begin{array}{c}\text { Celltype } \\
\text { (\% of viable cells) }\end{array}$ & $\begin{array}{c}\text { Subpopulation } \\
\text { (\% of parent) }\end{array}$ & $\begin{array}{c}6 \text { weeks } \\
\text { SM22 } \alpha-\text { hDTr } \\
\text { /ApoE } \%\end{array}$ & $\begin{array}{c}6 \text { weeks } \\
\text { SM22 } \alpha-\text { hDTr }^{+} \\
\text {/ApoE } \%\end{array}$ & $\begin{array}{l}18 \text { weeks } \\
\text { SM22 } \alpha-\text { hDTr } \\
\text { /ApoE } \%\end{array}$ & $\begin{array}{c}18 \text { weeks } \\
\text { SM22 }^{-h}-\mathrm{hDTr}^{+} \\
/ \text {/ApoE }^{-}\end{array}$ & $\mathbf{P}$ \\
\hline B-cells & & $6,54 \pm 3,61$ & $10,46 \pm 3,54$ & $4,71 \pm 1,60$ & $5,89 \pm 2,48$ & 0,0009 \\
\hline NK-Cells & & $0,36 \pm 0,17$ & $0,45 \pm 0,12$ & $0,33 \pm 0,29$ & $0,32 \pm 0,14$ & n.s. \\
\hline Granulocytes & & $53,54 \pm 6,22$ & $54,34 \pm 4,00$ & $50,41 \pm 5,84$ & $48,47 \pm 7,99$ & n.s. \\
\hline Monocytes & & $10,58 \pm 3,44$ & $12,91 \pm 3,68$ & $9,63 \pm 3,91$ & $10,37 \pm 3,06$ & n.s. \\
\hline & Ly6C low & $8,74 \pm 4,42$ & $11,97 \pm 3,61$ & $10,23 \pm 5,64$ & $14,52 \pm 6,32$ & n.s. \\
\hline & Ly6C high & $79,10 \pm 15,94$ & $74,13 \pm 14,44$ & $81,46 \pm 11,32$ & $75,88 \pm 10,64$ & n.s. \\
\hline T-cells & & $1,36 \pm 0,57$ & $1,72 \pm 0,71$ & $1,41 \pm 0,70$ & $1,66 \pm 0,51$ & n.s. \\
\hline
\end{tabular}


Chapter 4

Supplemental table 3. Primer list.

\begin{tabular}{|ccc|}
\hline Gene & Forward & Reverse \\
\hline B-actin & GGCACCACACCTTCTACAATG & GGGGTGTTGAAGGTCTCAAAC \\
\hline GAPDH & AGGTCGGTGTGAACGGATTTG & TGTAGACCATGTAGTTGAGGTCA \\
\hline $18 \mathrm{~s}$ & ACTTTTGGGGCCTTCGTGTC & GCCCAGAGACTCATTTCTTCTTG \\
\hline CNN1 & GCAGTGGACACACGCATTTT & AACAACTGGCCCCAAGACTC \\
\hline$\alpha$ SMA & CTGACAGAGGCACCACTGAA & CATCTCCAGAGTCCAGCACA \\
\hline
\end{tabular}




\section{Chapter 5}

Use of primary vascular smooth muscle cells to predict vascular calcification potential of patients: Development of a BioHybrid Assay

A.M.G. Jaminon, C. Akbulut, R. Kramann, E. Biessen, B. Mees, E. Natour, V.
Brandenburg, W. Jahnen-Dechent, J. Floege, J. Uitto, C.P. Reutelingsperger, L.J.
Schurgers
In preparation 


\begin{abstract}
Background: Vascular calcification is an active process and increases cardiovascular disease (CVD) risk. The current methods to detect vascular calcification are computed tomography and ${ }^{18} \mathrm{~F}-\mathrm{NaF}$ positron emission tomography. Clinical research into validated biomarkers is making progress but there is still no consensus on an appropriate marker for vascular calcification. We reason that the biomarker for vascular calcification is not a single plasma component but the collection of all plasma components and aimed to develop a cell-based assay to measure calcification (BioHybrid assay).

Methods: Human vascular smooth muscle cells (hVSMCs) were exposed to high calcium conditions and were challenged with different serum and plasma samples of patients susceptible for vascular calcification. Patient sera and plasma were derived from different cohort studies including chronic kidney disease (CKD) stages III, IV, V and VD, pseudoxanthoma elasticum and other CVD disease with high calcification score. Live hVSMC calcifying propensity was measured in a new developed assay using Fetuin A-Alexa 546.

Results: The BioHybrid assay was comparable to the gold-standard Ocresolphthalein method of calcification determination and is able to measure calcification development over time. The BioHybrid assay indicated that serum of dialysis patients before dialysis were more prone to calcify as compared to serum of the same patient after dialysis. Additionally, PXE, oral anticoagulation (OAC) and high $\mathrm{CAC}$ score patients showed greater calcification as compared to control.

Conclusion: We demonstrate a novel method for detecting vascular calcification in vitro. Using a Fetuin A fluorescent probe we have developed a real-time calcification assay that is able to give a quantifiable readout of calcification development over time in patients vulnerable for vascular calcification.
\end{abstract}




\section{Introduction}

Cardiovascular disease (CVD) is the largest killer in the world and globally CVDs account for approximately $30 \%$ of all-cause deaths annually ${ }^{1}$. Currently the majority of medical interventions for CVD occur post-event. Therefore, there is an unmet need of pre-event diagnostics to accurately predict susceptibility to cardiovascular events. Clinical research into validated biomarkers is making progress but is still insufficient to resolve professional consensus on appropriate markers ${ }^{2}$.

Vascular calcification is an active process that occurs within the vessel wall and increases the risk of cardiovascular disease ${ }^{3}$. Moreover, in the clinic coronary artery calcification (CAC) is measured by computed tomography (CT) as marker for cardiovascular burden. Calcification present at any vascular site relates to some 3.5 -fold increase for CVD events ${ }^{4}$. Additionally, vascular calcification progression is associated with a 17-fold increase of risk of myocardial infarction ${ }^{5}$. Therefore, means to measure vascular calcification amount or progression rate, promise to predict accurately patient risks for cardiovascular events.

The current methods to detect and assess calcification aim to visualize precipitated calcium salts in the vasculature such as $\mathrm{CT}$ and ${ }^{18} \mathrm{~F}-\mathrm{NaF}$ positron emission tomography (PET). Circulating biomarkers reflecting the patient's propensity to develop and aggravate vascular calcification have not been identified so far. In this paper we reason that the circulating biomarker for vascular calcification is not a single plasma component but the collection of all plasma components. As readout of this composite biomarker we selected in vitro calcification mediated by vascular smooth muscle cells, which are considered to be the cellular protagonists in vascular calcification. This paper describes the BioHybrid calcification assay, a cell-based assay for measuring the composite biomarker of vascular calcification. We measured the BioHybrid response for plasma samples of chronic kidney disease (CKD) patients, pseudoxanthoma elastica (PXE) patients and CVD patients of whom the Agatston score of CAC was measured. We show that there is a strong and significant correlation between the Agatston score and the BioHybrid calcification readout. We conclude that the BioHybrid calcification assay is an informative strategy to determine the patient's vascular calcification propensity.

\section{Methods}

\section{Human vascular smooth muscle cell culturing and characterization}

Human vascular smooth muscle cells (hVSMCs) were isolated from nonatherosclerotic aortas of surgical biopsies in accordance with MUMC+ research and diagnostic procedure. In short, human vascular tissue samples were transported in icecold serum free M199 medium containing 1\% Penicillin/ Streptomycin (PS) and washed in phosphate buffered saline (PBS). Intima, fat and connective tissue was removed before cutting the vascular tissue into small fragments and placing each into a laminin (\#L2020, Sigma) coated 6-well plates, with the intima facing down. The pieces were covered with M199 medium containing 20\% fetal bovine serum (FBS), 1\% PS and 1\% Amphotericin B (\#15290-026, Gibco) and placed into an incubator. When outgrowing cells reached confluency, they were passaged to laminin coated T25 flasks and tested for mycoplasma. hVSMCs were cultured in M199 medium (Gibco, Bleiswijk, the Netherlands) 
supplemented with 20\% FBS and 1\% penicillin/streptomycin (P/S) and used between passages 5-10 for our experiments.

To characterize cells (supplemental Figure 2), hVSMCs were fixed in $4 \%$ paraformaldehyde before blocking in $2 \% \mathrm{BSA}, 0.1 \%$ triton in PBS for one hour. Primary antibodies were incubated overnight at $4{ }^{\circ} \mathrm{C}$ : alpha-Smooth Muscle Actin ( $\alpha$ SMA) 1:200 (DAKO, M0851), phosphorylated-Myosin Light Chain (pMLC) 1:200 (Cell Signaling, 3675S), Calponin (CNN1) 1:200 (Abcam, ab46794), Smooth Muscle protein 22-alpha (SM22a) 1:200 (Abcam, ab14106), S100 calcium-binding protein A4 (S100A4) 1:200 (DAKO, A5114). Cells were incubated with secondary antibodies for one hour at room temperature: anti-mouse FITC 1:250 (Hycult Biotech, HP2001), anti-rabbit fluorescein isothiocyanate (FITC) (DAKO, F0205). Before imaging cells were incubated with 4',6diamidino-2-phenylindole (DAPI) for one minute. Imaging was performed on the Cytation 3 system (BioSPX, Abcoude, The Netherlands).

\section{hVSMC mediated calcification}

For calcification experiments, hVSMCs were seeded at a density of $1.3 \times 10^{\wedge} 4$ cells per $\mathrm{cm}^{2}$. After 24h, hVSMCs were cultured in calcification medium (M199, 5\% human serum/plasma, $1 \% \mathrm{P} / \mathrm{S}$ and $3.6 \mathrm{mM} \mathrm{Ca}^{2+}$ ) up to 14 days. Plasma samples were further supplemented with Hirudin to prevent clotting of the samples.

\section{Real-time kinetic assay}

For real-time calcification measurement, Fetuin A-Alexa $546(3 \mu \mathrm{g} / \mathrm{ml}$; kindly provided by Prof. Dr. W. Jahnen-Dechent, RWTH Aachen, Aachen, Germany) and Hoechst $(1 \mu \mathrm{g} / \mathrm{ml})$ were supplemented at the start of the experiment. At various time points after calcification induction, red fluorescent protein (RFP) and DAPI channel (cell count) were imaged up to 14 days. After calcification was detected, cells were imaged regularly to follow calcification progression. Imaging was done with the Cytation 3 system (BioSPX, Abcoude, The Netherlands) and the Gen5 software (BioTek).

\section{End-point assay}

For end-point kinetic measurement the O-cresolphthalein method (Randox, London, United Kingdom) was used. The O-cresolphthalein assay was carried out according to manufacturer's instructions, after solubilising mineral deposits in $0.1 \mathrm{M}$ $\mathrm{HCl}$.

\section{Patient serum/ plasma preparation}

Patient serum and plasma samples were obtained from different cohort studies. Patient samples that were included were: CKD5D, CKD 3-5, PXE, high and low CAC score patients, oral anticoagulation (OAC) patients and aortic valve calcification (AVC) patients that received vitamin $\mathrm{K} 1$ supplementation. $\mathrm{CKD}$ patients are known to be prone to develop vascular calcification ${ }^{6}$. PXE patients are known to have a monogenetic liver disorder of the ATP Binding Cassette Subfamily C Member 6 (ABCC6), which is characterized by dystrophic mineralisation of soft connective tissues and elastic structures in several organs ${ }^{7}$. Additionally, we used samples from individuals enrolled with AVC that were supplemented with vitamin $\mathrm{K} 1$ or placebo ${ }^{8}$. Last, serum and plasma of healthy controls was obtained as negative control. This research was approved by the Ethics Committee of the Klinikum Aachen and Sidney Kimmel Medical College at 
Thomas Jefferson University, Philadelphia and conducted in accordance with the Declaration of Helsinki.

\section{Dephosphorylated-uncarboxylated matrix Gla protein (dp-ucMGP) measurement}

Plasma dp-ucMGP levels were determined using the commercially available IVD CE-marked chemiluminescent InaKtif MGP assay on the IDS-iSYS system (IDS, Boldon, UK). In brief, patient samples and internal calibrators were incubated with magnetic particles coated with murine monoclonal antibodies against dp-MGP, acridinium-labelled murine monoclonal antibodies against ucMGP, and an assay buffer. The magnetic particles were captured using a magnet and washed to remove any unbound analyte. Trigger reagents were added; the resulting light emitted by the acridinium label was directly proportional to the level of dp-ucMGP in the sample. The within-run and total variations of this assay were $0.8-6.2 \%$ and $3.0-8.2 \%$, respectively. The assay measuring range was between 300 and $12,000 \mathrm{pmol} / \mathrm{L}$ and was linear up to $11,651 \mathrm{pmol} / \mathrm{L}^{8}$. All assays were performed by Coagulation Profile laboratories, department of Biochemistry, Maastricht, the Netherlands.

\section{Data analysis and statistics}

Real-time calcification development over time was performed by using Gen5 version 2.9 (BioTek). Area of Alexa 546 signal was determined and normalized against cell count. Data are presented as mean \pm standard deviation (SD). Non-parametric MannWhitney U test was performed for comparison between two groups. For more than two groups, significance was determined using one-way analysis of variance (ANOVA) with comparison between groups by Dunn's multiple comparison test. For correlation determination a linear regression model and $\mathrm{R}^{2}$ was determined. Statistical significance was defined as $\mathrm{P} \leq 0.05(*), \mathrm{P} \leq 0.01(* *), \mathrm{P} \leq 0.001(* * *)$ and $\mathrm{P} \leq 0.0001(* * * *)$.

\section{Results}

\section{BioHybrid calcification assay}

Thus far, measurement of in vitro calcification is only possible via end-point assays using alizarin red or O-cresolphthalein quantification. We designed a novel in vitro calcification assay using hVSMCs in which calcification development was followed in time by fluorescently labeled Fetuin A. To test the robustness of our live calcification assay we compared the BioHybrid assay with the O-cresolphthalein method, that measures calcification as calcium/protein ratio. We found a strong correlation $\left(\mathrm{R}^{2}=0.72\right)$ between Fetuin A-Alexa 546 fluorescence per cell $(\% \mathrm{RFP} /$ cell count) and O-cresolphthalein $\left(\mu \mathrm{g} \mathrm{Ca}^{2+} / \mu \mathrm{g}\right.$ protein; Figure 1A; representative images Figure 1D).

\section{Variability}

A great advantage of using Fetuin A-Alexa 546 is that it can be used in real time, measuring calcification progression in vitro over time. The use of Fetuin A-Alexa 546 unfolds novel possibilities that can provide a quantifiable readout of calcification during one assay. We tested a variety of both serum and calcium conditions to establish the optimal parameters for our assay. We found that the use of $5 \%$ serum was optimal for a robust increase in calcification (Figure 1B). Under $0.5 \%$ and $2.5 \%$ FBS conditions, 


\section{Chapter 5}

development of calcification was too rapid (Figure 1B) and when using 10\% FBS calcification formation was low (data not shown), as was shown previously ${ }^{9}$. Another parameter that is not uniform in calcification assays is the used concentration of calcium to trigger calcification development. All our assays were performed with $3.6 \mathrm{mM}$ of calcium chloride $\left(\mathrm{CaCl}_{2}\right)$. Higher concentrations of $\mathrm{CaCl}_{2}(4.5 \mathrm{mM}$ and $5.4 \mathrm{mM})$ had a much more rapid development of calcification, comparable to lower serum levels (supplemental Figure 1). Hence, we used $3.6 \mathrm{mM} \mathrm{CaCl}_{2}$ and $5 \%$ serum for our BioHybrid assay.

Since we developed the BioHybrid system to test clinical samples, we investigated whether human serum or plasma could allow for calcification development. Comparing FBS with either a pool of healthy control serum/ plasma or the individual serum/ plasma from each of our healthy controls we did not find differences in hVSMC calcification (Figure 1E). This indicates that serum as well as plasma samples can be used to measure calcification with our assay.

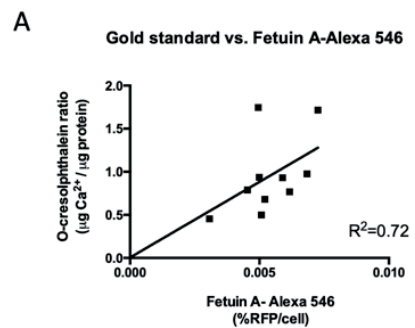

B
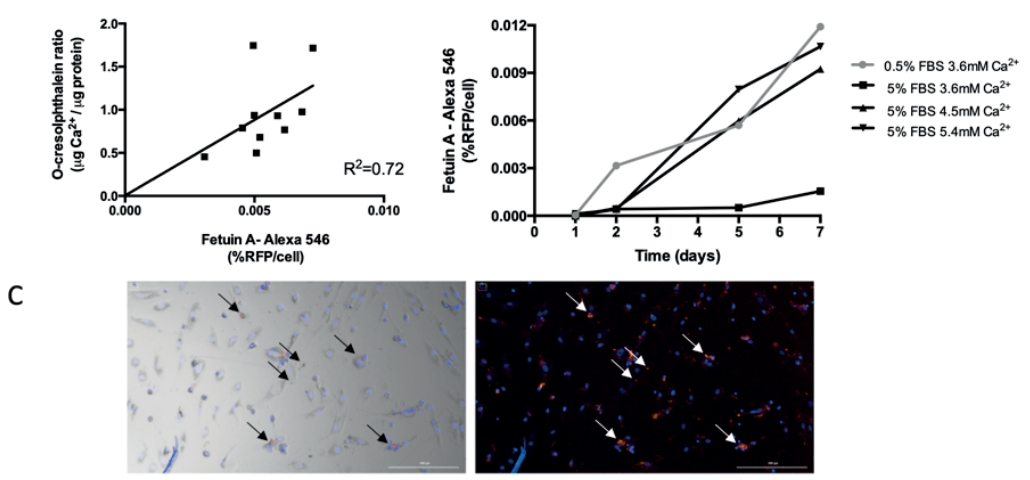

D

$0.5 \%$ FBS
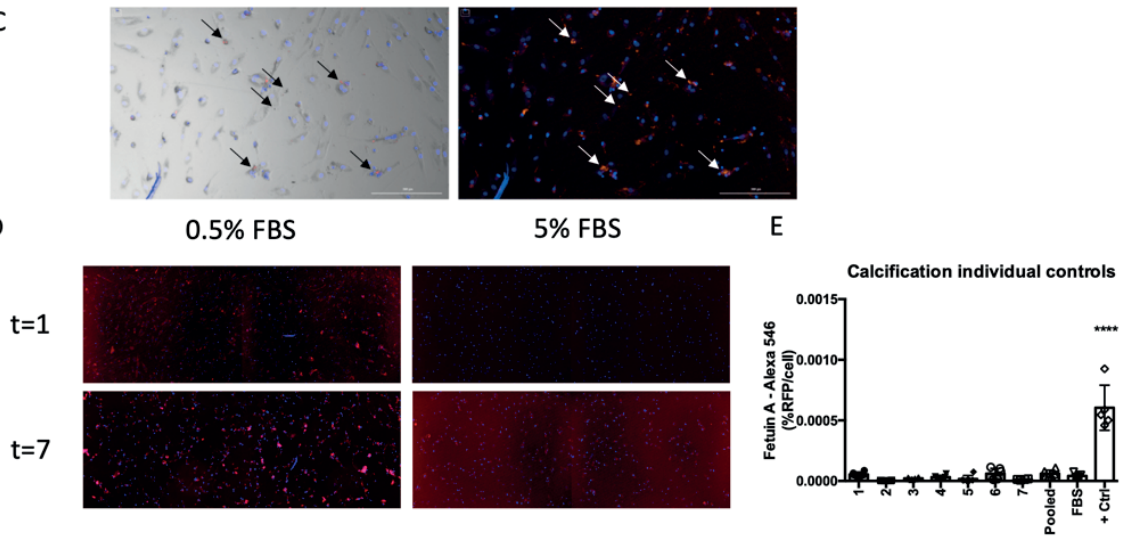

Figure 1. BioHybrid calcification assay. (A) Live calcification assay compared to the O-cresolphthalein method. (B) RFP signal over time, to determine the best calcifying conditions. (C) micro calcification can be determined with the Fetuin A-Alexa546 probe. (D) Representative images of the BioHybrid calcification assay at $\mathrm{t}=1$ and $\mathrm{t}=7$ days. $0.5 \%$ FBS concentration give rise to a strong positive signal while $5 \%$ FBS only shows mild calcification after 7 days. (E) Calcification assay comparing FBS to serum of healthy individuals and a pooled condition. $* * * * \mathrm{p}<0.0001$ 


\section{Calcification propensity of CKD5D serum}

We measured the calcifying potential of serum of patients diagnosed with CKD5D. After 3 days there was a distinguishable difference between serum of CKD5D patients and healthy controls (Figure 2A and 2B). After 6 days, the CKD5D sera continued to increase in vitro calcification whereas the control population maintained lower levels of calcification (Figure 2A and 2B).

Next, we interrogated whether dialysis would have an effect on the calcification propensity of VSMCs. When analyzing the rate of calcification between two time points (72 hours apart), we show that pre-dialysis serum samples induce a significantly increased rate of VSMC calcification, compared to post-dialysis of the same patients (Figure 2C). Further, the BioHybrid system is notably responsive to dialysis after 3 and 6 days (Figures 2E-G). Moreover, serum from 5 out of the 7 patients had a significantly lower induction of calcification following dialysis treatment (Figure 2D). Serum from one patient had no significant difference and intriguingly one other patient showed a significant increase in in vitro VSMC calcification after dialysis (Table 1).

A

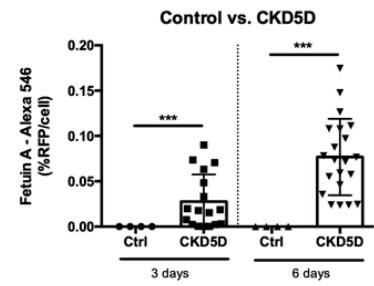

B
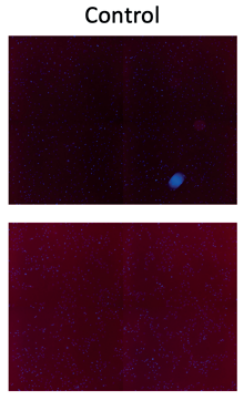

CKD5D
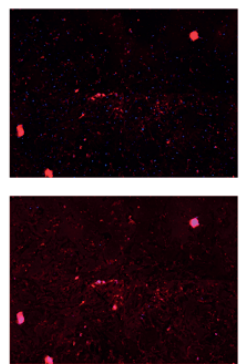

c

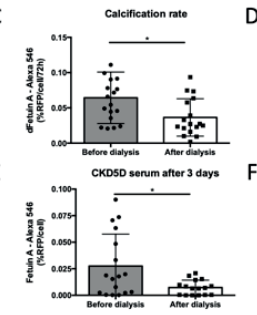

G
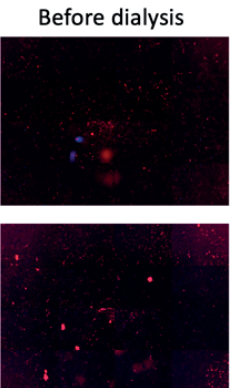

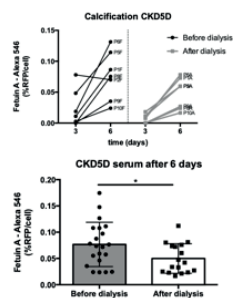

After dialysis
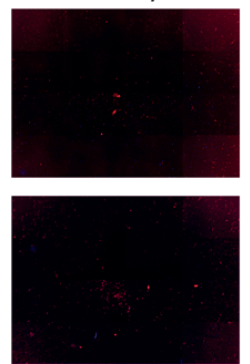

Figure 2. Calcification in CKD5D patients. (A) Serum of CKD5D patients tested in the BioHybrid assay. A significant increase of calcification propensity was found at $t=3$ and $t=6$ days. (B) Representative images of the calcification assay, control vs. CKD5D serum. (C) The effect of dialysis on the calcification rate. Serum of patient before dialysis has a significant higher calcification rate as compared to the same patients after dialysis. (D) Calcification increase from $\mathrm{t}=3$ to $\mathrm{t}=6$ days in the assay. (E) The effect of dialysis on calcification at $\mathrm{t}=3$. As significant increase in calcification was found in serum before dialysis. (F) The effect of dialysis on calcification at $\mathrm{t}=6$. As significant increase in calcification was found in serum before dialysis. (G) Representative images of the calcification assay of serum before and after dialysis. ${ }^{*} \mathrm{p}<0.05 ;{ }^{* * *} \mathrm{p}<0.001$ 
Table 1. Calcification response to dialysis. Positive indicates that serum before dialysis has more calcification compared to serum of the same patient after dialysis.

\begin{tabular}{|l|l|}
\hline Response to dialysis & Percentage (\%) \\
\hline Positive & 71.4 \\
\hline No change & 14.3 \\
\hline Negative & 14.3 \\
\hline
\end{tabular}

\section{Calcification propensity of CKD 3-5 serum}

We compared hVSMC calcification induction of patient serum from CKD stages 3, 4 and 5 against a pool of healthy donors. As seen figure $3 \mathrm{~A}$ the healthy control had the lowest rate of calcification development per hour. CKD5 showed the highest calcification rate followed by CKD4 and CKD3 respectively ( $\mathrm{p}=0.022$; CKD5 vs. Ctrl). We did observe a positive trend in calcification rate of CKD patients, which was statistically significant compared to control $(\mathrm{p}=0.039)$. By day 7 , the most significant in vitro calcification was observed in CKD5 patients, followed by CKD4, CKD3, and the lowest in healthy controls (Figures $3 \mathrm{~B}$ and $3 \mathrm{C}$; not significant).

A

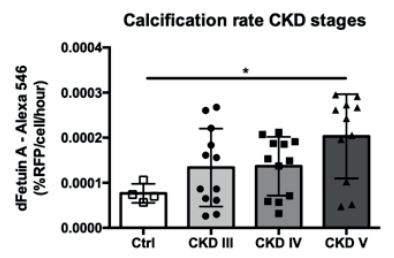

B

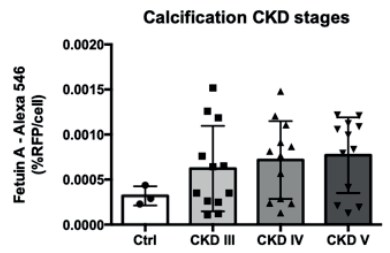

C

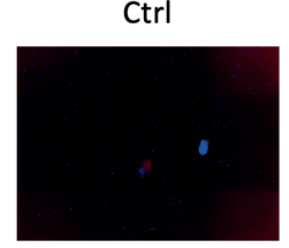

CKD IV

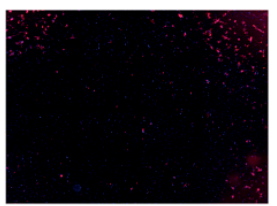

CKD III

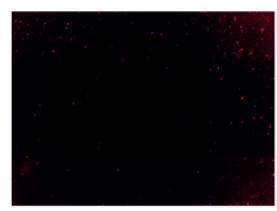

CKD V

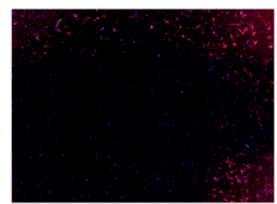

Figure 3. Calcification in CKD stages III-V. (A) Calcification rate in the BioHybrid calcification assay. A significant increase was found in serum of CKDV patient compared to control. (B) Calcification signal at $\mathrm{t}=7$. (C) Representative images at $\mathrm{t}=7$ days. ${ }^{*} \mathrm{p}<0.05$ 


\section{Calcification propensity of PXE plasma}

PXE is a connective tissue disorder that results in elastic tissue mineralization and calcium deposition. PXE patients display reduced anti-mineralization capacity that results in extensive ectopic calcification of the skin, eyes and cardiovascular system. We found that plasma from PXE patients calcified much faster compared to both a healthy control plasma pool and spouse control plasma (Figure 4A and 4B). This difference was found to be significant between the PXE group and spouse controls (Figure 4A; $\mathrm{p}<0.001)$.

A

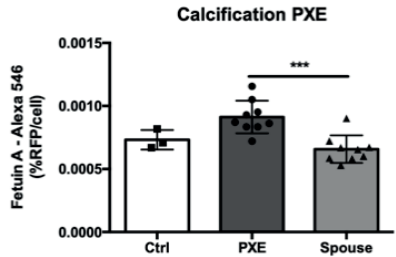

B Ctrl

PXE

Spouse
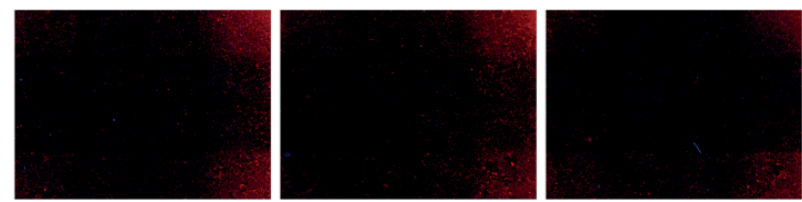

Figure 4. Calcification in PXE patients. (A) Calcifciation of plasma of PXE patients compared to their spouses and healthy controls. A significant increase in calcification was found PXE patients compared to their spouses. (B) Representative images of the calcification. $* * * p<0.001$

\section{Calcification propensity of low and high Agatston score serum}

Next, we tested whether our BioHybrid system is able to detect differences in in vitro calcification of patients with high and low CAC scores. For this we used serum from patients which were high $(>1500)$ or low $(<50)$ in Agatston score. In vitro calcification was significantly higher for serum from patients with a high Agatston scoring compared to serum from patients with a low Agatston score (Figure 5A and 5C; $\mathrm{p}=0.002$ ). A significant correlation was observed between Agatston score and VSMC calcification in our BioHybrid assay. Dividing patients in high and low Agatston score, those with a high Agatston score displayed a significant correlation with in vitro calcification (Figure $5 \mathrm{~B} ; \mathrm{R}^{2}=0.68$ ). No such correlation was observed with patients in the low Agatston group (data not shown). 

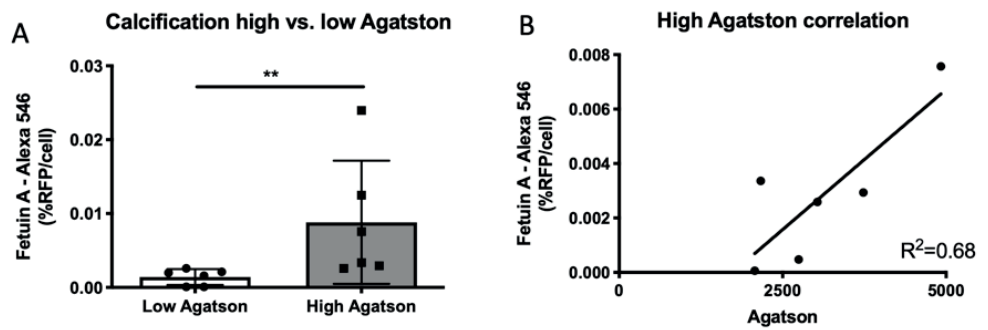

C
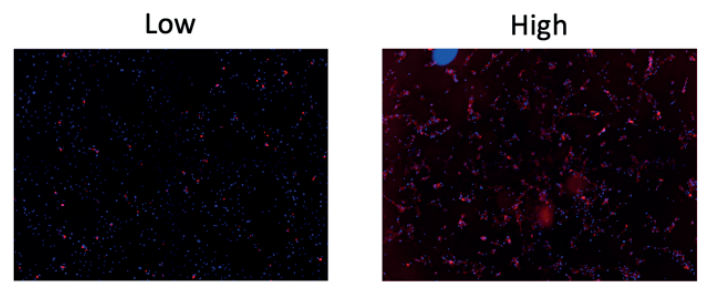

Figure 5. Calcification in low and high Agatston score patients. (A) Calcification of serum from low vs. high Agatston score patients. A significant increase in calcification was found in serum of patients determined to be high compared to low Agatston score. (B) Correlation of Agatston score to calcification in our BioHybrid assay. Only a significant correlation was found in the high Agatston score group $\left(\mathrm{R}^{2}=0.68\right)$. (C) Representative images of calcification from patients with low vs. high Agatston score. ${ }^{* *} \mathrm{p}<0.01$

\section{Calcification propensity of OAC plasma}

Both patients and healthy volunteers on vitamin $\mathrm{K}$ antagonists (VKAs) displayed increased in vitro calcification (Figure 6A and 6B) when compared to no anticoagulation use (normalized INR). Additionally, healthy volunteers supplemented with vitamin K2 (MK7) show decreased calcification (Figure 6B). Complementary, patients that were using VKAs had lower turbidity time, while MK7 supplementation increased this (Figure 6C).

A

Plasma one patient (24h later)

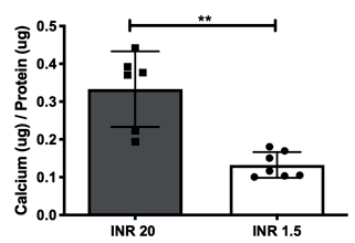

Healthy volunteer on Coumarin or Vitamin $\mathbf{K}$

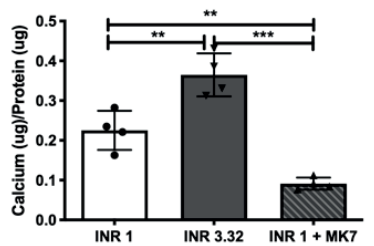

Patient on Coumarin or Vitamin K

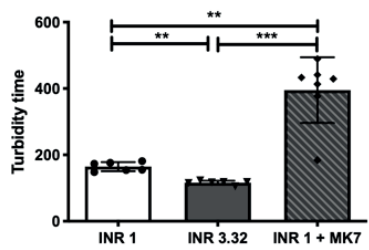

Figure 6. Calcification of plasma of OAC patients. (A) Calcification of plasma of a patient on VKA vs. the same patients with a normalized INR. A significant decrease in calcification was found in plasma with a normalized INR. (B) Calcification of plasma from a healthy volunteer taking VKA or MK7 supplementation. A significant increase in calcification was found when taking VKA while a decrease in calcification was found when taking MK7 supplementation. (C) Turbidity time of plasma from a patient taking VKA or MK7 supplementation. A significant decrease in turbidity time was found when taking VKA while an increase in turbidity time was found with MK7 supplementation. ${ }^{* *} \mathrm{p}<0.01 ; * * \mathrm{p}<0.001$ 


\section{Calcification propensity of AVC serum with vitamin K1 treatment}

Finally, we interrogated serum samples from AVC patients that were supplemented with vitamin $\mathrm{K}$ for one year ${ }^{8}$. After 12 months of supplementation with vitamin $\mathrm{K}$, serum from patients showed reduced in vitro calcification (Figure 7A). Conversely, serum from patients given placebo for one year exhibited increased calcification (Figure 7A and 7E; $\mathrm{p}=0.06$ ). Exogenous supplemented vitamin $\mathrm{K}$ to serum from patients in the placebo group had no effect on calcification also when compared to patients treated for one year with vitamin K (Figure 7B).

Finally, patient plasma was used to determine the vitamin $\mathrm{K}$ status of AVC patients using dp-ucMGP as biomarker (Figure 7C). Dp-ucMGP was significantly decreased in the patients supplemented with vitamin $\mathrm{K} 1(\mathrm{p}<0.0001)$. Comparing the delta change in Agatston score between baseline and one year treatment, no differences in either carotid artery or aortic valve calcification between vitamin $\mathrm{K} 1$ or placebo could be observed (Figure 7D).
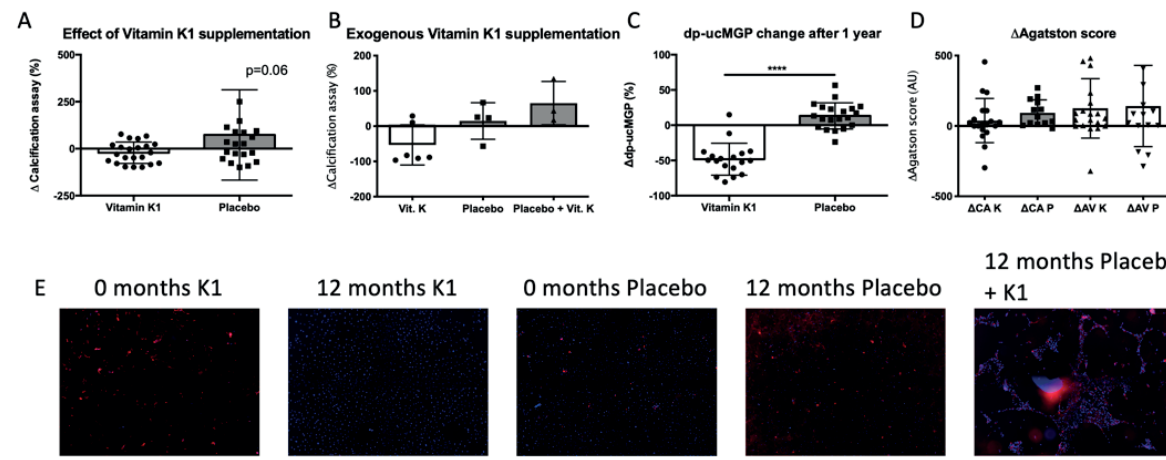

12 months Placebo

$+\mathrm{K} 1$

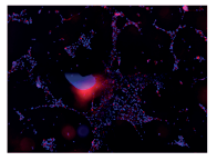

Figure 7. Calcification of AVC patients. (A) Calcification effect of 1 year Vitamin K supplementation vs. placebo. 12 months vitamin $\mathrm{K}$ supplementation decreased calcification while 1 year placebo supplementation increased calcification (not significant). (B) the effect of exogenous Vitamin K supplementation. Addition of exogenous Vitamin K did not decrease calcification as 1 year vitamin K supplementation did. (C) Dp-ucMGP change after one year of supplementation. Dp-ucMGP levels significantly decreased after 1 year of vitamin $\mathrm{K}$ supplementation. (D) Changes in Agatston score after one year of supplementation. (E) Representative images of calcification from patients taking Vitamin K or placebo supplementation. $* * * * p<0.0001$

\section{Discussion}

In vivo determination of cardiovascular burden using vascular calcification is challenging, costly, and exposes patients to radiation. Direct measures for assessing calcification are CT or ${ }^{18} \mathrm{~F}-\mathrm{NaF}$ Positron Emission Tomography (PET). Detection of vascular remodeling is based upon biomechanical properties of the vessel wall such as pulse wave velocity and intima-media thickness. Both in vivo measurements are limited to late stages of vascular calcification and are thus a marker for cardiovascular burden. Detecting vascular calcification at an early stage would allow to intervene before vascular disease has been established, Therefore, utilising serum of patients to detect vascular calcification at an early stage would provide a unique clinical tool. Here we demonstrate the in vitro use of fluorescently labeled Fetuin A as imaging tool to follow calcification progression real-time and to predict patient susceptibility for developing 
calcification. We verified our assay using serum from patients with vascular calcification progression.

The gold standard for measuring in vitro calcification, is a singular time-point read, based on a subjective time-point assessment of abundant calcification being present. The two methods that are predominantly used for determining calcification are Alizarin Red staining and O-cresolphthalein. Additionally, the rate in which serum calcifies, dependent on factors present in serum, can be assessed using the $T_{50}$ assay that measures the propensity of serum to calcify under non-physiologic conditions ${ }^{10}$. $\mathrm{T}_{50}$ results have shown to be associated with cardiovascular and all-cause mortality and aortic stiffening in renal patients ${ }^{11-13}$. Yet, this assay is mainly useful for serum of CKD patients, and does not take into account the vascular component. All current methods for calcification determination are end-point assays and there is need of a method capable of quantifying calcification rate in real-time. We found a strong and significant correlation between the O-cresolphthalein method and the BioHybrid assay. More specifically, in the early stages of in vitro calcification detection, the Fetuin A probe was superior and showed greater sensitivity. End-point assays require multiple replicates to record calcification development over time. With the BioHybrid assay we exploit livecell imaging, enabling us to follow calcification development of one sample over time. Severe renal artery, aortic calcification and aortic valve calcification is symptomatic in CKD patients ${ }^{14}$. Additionally, up to $93 \%$ of CKD patients on dialysis display vascular calcification ${ }^{15,16}$. Vascular calcification is also found in patients that suffer from PXE. PXE is a monogenetic liver disorder characterized by a loss-of-function mutation in the ABCC6 gene. PXE patients display reduced plasma anti-mineralization capacity due to lower Fetuin A and MGP activity. Additionally, they display extensive ectopic calcification which primarily manifests in the skin, the eye and cardiovascular system. PXE is metabolic disorder and thus ideally suitable for our BioHybrid screening ${ }^{7,17}$.

We found that in vitro calcification was increased for both CKD and PXE patient serum/ plasma. CKD5D serum induced extensive calcification after three days, further progressing after six days whereas calcification progression of serum from CKD 3,4 and 5 patients was significantly slower. This is in line with the less in vivo calcification that is found in the clinical situation with the different stages of CKD ${ }^{18-20}$. Additionally, pre-dialysis serum was shown to increase calcification development, indicative of altered blood components caused by dialysis. However, not every dialysis patient had a similar response in the Biohybrid assay. One patient did not show a preand post-dialysis difference, while one patient showed increased calcifying potential post-dialysis. This indicates that not all patients share the same blood components and respond differently in our assay, while they undergo dialysis and are having CKD. In our assay, PXE serum showed increased calcification when compared to spouse controls. Interestingly the constituents of PXE have been already demonstrated to interfere within ordinary elastic fiber deposition ${ }^{21}$.

Finally, the Biohybrid assay enables us to stratify amongst susceptible patients based on their effect of in vitro calcification and could aid in the discovery of dialysable or systemic factors that determine in vivo calcification.

CAC score is evaluated as the gold-standard clinical predictor for the accumulation and progression of vascular calcification over time ${ }^{5,22}$. Additionally, CAC score is used to identify high-risk individuals that need immediate medical attention or 
intervention ${ }^{23}$. Vitamin $\mathrm{K}$ supplementation is regarded as one of the solutions to hold the progression of vascular calcification. In particular, in patients that are on VKA treatment ${ }^{24}$. Indeed we show that patients as well as healthy volunteers have a high calcifying propensity when using VKAs. Additionally, when supplemented with vitamin K2 there is a decreased calcification tendency. This is confirmed by the T50 measurement that determines turbidity time of patient plasma.

Serum from patients with high CAC scores developed more in vitro calcification as compared to low CAC score individuals. We further observed a positive correlation of calcification development within the high CAC score group which was non-existing in the low CAC group. Additionally, serum of patients that received vitamin $\mathrm{K}$ supplementation for 12 months showed lower calcification development as compared to placebo controls. Interestingly, although all patients' cardiovascular status continued to decline the placebo group had on average a 25\% increase in Agatston scoring during this year, whereas the vitamin $\mathrm{K}$ group had only $12 \%$ increase. These results indicate that the BioHybrid assay can predict vascular calcification development and is sensitive to distinguish highly susceptible patients. Moreover, serum after one-year treatment with vitamin $\mathrm{K}$ resulted in lower in vitro calcification which associated with lower progression of Agatston score in vivo.

Dp-ucMGP is a biomarker that has been shown to associate with vascular calcification $^{4,25}$. Vitamin K supplementation decreased dp-ucMGP levels in all patients, yet this was not reflected in the development of vascular calcification. We reason that dp-ucMGP only reflects as one biomarker involved in calcification. Thus, dp-ucMGP reflects the current status of the protein in the plasma pool, while the BioHybrid assay employs the whole blood compartment. We hypothesize that the BioHybrid platform can become an assay for clinical risk assessment of vascular calcification progression.

In conclusion, we demonstrate a novel method for detecting vascular calcification in vitro. Using a Fetuin A fluorescent probe we have developed a real-time calcification assay that is able to give a quantifiable readout of calcification development over time. We applied our assay to patients with varying calcification levels and demonstrated its application as an indicative readout based on cardiovascular status. Further the sensitivity of this assay has been demonstrated in response to dialysis, vitamin $\mathrm{K}$ treatment, as well as both metabolic and non-metabolic disorders that directly affect cardiovascular status. We propose wide scale application of this assay in larger cohorts to further validate application and efficacy of this tool as well as in application as a low-cost, non-invasive cardiovascular diagnostic. 


\section{Chapter 5}

\section{References}

1. GBD 2017 Risk Factor Collaborators. Global, regional, and national comparative risk assessment of 84 behavioural, environmental and occupational, and metabolic risks or clusters of risks for 195 countries and territories, 1990-2017: a systematic analysis for the Global Burden of Disease Study 2017. Lancet 392, 1923-1994 (2018).

2. Khambhati, J. et al. The art of cardiovascular risk assessment. Clin Cardiol 41, 677-684 (2018).

3. Schurgers, L. J. et al. Initiation and Propagation of Vascular Calcification Is Regulated by a Concert of Platelet- and Smooth Muscle Cell-Derived Extracellular Vesicles. Front Cardiovasc Med 5, 36 (2018).

4. Rennenberg, R. J. M. W. et al. Vascular calcifications as a marker of increased cardiovascular risk: a meta-analysis. Vasc Health Risk Manag 5, 185-197 (2009).

5. Raggi, P., Callister, T. Q. \& Shaw, L. J. Progression of coronary artery calcium and risk of first myocardial infarction in patients receiving cholesterol-lowering therapy. Arteriosclerosis, Thrombosis, and Vascular Biology 24, 1272-1277 (2004).

6. Garimella, P. S. \& Sarnak, M. J. Cardiovascular disease in CKD in 2012: moving forward, slowly but surely. Nat Rev Nephrol 9, 69-70 (2013).

7. Uitto, J., Li, Q. \& Jiang, Q. Pseudoxanthoma elasticum: molecular genetics and putative pathomechanisms. J. Invest. Dermatol. 130, 661-670 (2010).

8. Brandenburg, V. M. et al. Slower Progress of Aortic Valve Calcification With Vitamin K Supplementation: Results From a Prospective Interventional Proof-of-Concept Study. Circulation 135, 2081-2083 (2017).

9. Reynolds, J. L. et al. Human vascular smooth muscle cells undergo vesicle-mediated calcification in response to changes in extracellular calcium and phosphate concentrations: a potential mechanism for accelerated vascular calcification in ESRD. J. Am. Soc. Nephrol. 15, 2857-2867 (2004).

10. Pasch, A. et al. Nanoparticle-Based Test Measures Overall Propensity for Calcification in Serum. $J$. Am. Soc. Nephrol. 23, 1744-1752 (2012).

11. Smith, E. R. et al. Serum calcification propensity predicts all-cause mortality in predialysis CKD. $J$. Am. Soc. Nephrol. 25, 339-348 (2014).

12. Keyzer, C. A. et al. Calcification Propensity and Survival among Renal Transplant Recipients. $J$. Am. Soc. Nephrol. 27, 239-248 (2016).

13. Dahle, D. O. et al. Serum Calcification Propensity Is a Strong and Independent Determinant of Cardiac and All-Cause Mortality in Kidney Transplant Recipients. Am. J. Transplant. 16, 204-212 (2016).

14. O'Shaughnessy, M. M., Liu, S., Montez-Rath, M. E., Lafayette, R. A. \& Winkelmayer, W. C. Cause of kidney disease and cardiovascular events in a national cohort of US patients with end-stage renal disease on dialysis: a retrospective analysis. Eur. Heart J. (2018). doi:10.1093/eurheartj/ehy422

15. Nitta, K. et al. Assessment of coronary artery calcification in hemodialysis patients using multidetector spiral CT scan. 27, 527-533 (2004).

16. Wang, X.-R., Zhang, J.-J., Xu, X.-X. \& Wu, Y.-G. Prevalence of coronary artery calcification and its association with mortality, cardiovascular events in patients with chronic kidney disease: a systematic review and meta-analysis. 41, 244-256 (2019).

17. Uitto, J., Li, Q., van de Wetering, K., Váradi, A. \& Terry, S. F. Insights into Pathomechanisms and Treatment Development in Heritable Ectopic Mineralization Disorders: Summary of the PXE International Biennial Research Symposium-2016. J. Invest. Dermatol. 137, 790-795 (2017).

18. Russo, D. et al. Progression of coronary artery calcification and cardiac events in patients with chronic renal disease not receiving dialysis. Kidney Int. 80, 112-118 (2011).

19. Stavroulopoulos, A. et al. Evolution of coronary artery calcification in patients with chronic kidney disease Stages 3 and 4, with and without diabetes. Nephrol. Dial. Transplant. 26, 2582-2589 (2011).

20. Lamarche, M. C., Hopman, W. M., Garland, J. S., White, C. A. \& Holden, R. M. Relationship of coronary artery calcification with renal function decline and mortality in predialysis chronic kidney disease patients. Nephrol. Dial. Transplant. 34, 1715-1722 (2019).

21. Gheduzzi, D. et al. Matrix Gla protein is involved in elastic fiber calcification in the dermis of pseudoxanthoma elasticum patients. Lab. Invest. 87, 998-1008 (2007).

22. Raggi, P. Cardiovascular disease: Coronary artery calcification predicts risk of CVD in patients with CKD. Nat Rev Nephrol 13, 324-326 (2017).

23. Yamamoto, H., Kitagawa, T. \& Kihara, Y. Clinical Implications of the Coronary Artery Calcium Score in Japanese Patients. J. Atheroscler. Thromb. 21, 1101-1108 (2014). 
24. van Gorp, R. H. \& Schurgers, L. J. New Insights into the Pros and Cons of the Clinical Use of Vitamin K Antagonists (VKAs) Versus Direct Oral Anticoagulants (DOACs). Nutrients 7, 95389557 (2015).

25. Chatrou, M. L. L., Winckers, K., Hackeng, T. M., Reutelingsperger, C. P. \& Schurgers, L. J. Vascular calcification: The price to pay for anticoagulation therapy with vitamin K-antagonists. YBLRE 26, 155-166 (2012). 


\section{Chapter 6}

Matrix Gla protein is an independent predictor of both intimal and medial vascular calcification in chronic kidney disease

A.M.G Jaminon, L. Dai, A.R. Qureshi, P. Evenepoel, J. Ripsweden, M. Söderberg, A. Witasp, H. Olauson, L.J. Schurgers*, P. Stenvinkel*

Sci Rep. Nature Publishing Group; 2020 Apr 20;10(1):6586-9. 


\title{
Chapter 6
}

\begin{abstract}
Matrix Gla protein (MGP) is a potent inhibitor of vascular calcification (VC) and requires carboxylation by vitamin $\mathrm{K}$ to exert calcification inhibition. Chronic kidney disease (CKD) patients undergo early vascular aging often involving extensive VC. The present cross-sectional study investigated the association between circulating dp-ucMGP levels, MGP expression in vascular tissue and MGP polymorphisms. In 141 CKD stage 5 patients, CAC score was significantly increased in the highest tertile of dp-ucMGP $(\mathrm{p}=0.002)$, and a high medial VC score was associated with elevated dp-ucMGP levels. MGP vascular expression was associated with increased circulating dp-ucMGP and CAC scores. MGP SNP analysis revealed that patients homozygous for the $\mathrm{C}$ allele of the rs1800801 variant had a higher CAC score (median 15 [range 0-1312]) compared to patients carrying a $\mathrm{T}$ allele (median 0 [range 0-966] AU). These results indicate that plasma levels of dp-ucMGP are an independent predictor of increased VC in CKD5 patients and correlate with both higher CAC scores and degree of medial calcification. Additionally, high vascular expression of MGP was associated with higher CAC scores and plasma dp-ucMGP levels. Taken together, our results support that MGP is involved in the pathogenesis of VC.
\end{abstract}




\section{Introduction}

Matrix Gla protein (MGP) is a vitamin $\mathrm{K}$ dependent protein (VKDP) that is involved in the inhibition of vascular calcification (VC). MGP is small secretary protein $(14 \mathrm{kD})$ that is primarily secreted by vascular smooth muscle cells (VSMCs) in the arterial wall ${ }^{1}$. MGP contains five Glu residues that require carboxylation to become activated and to fulfill its calcification inhibitory function. This carboxylation step cannot take place in the absence of vitamin $\mathrm{K}$, which has an unequivocal role in driving this post-translational step ${ }^{2,3}$. Vitamin $\mathrm{K}$ is a co-factor for the enzyme $\gamma$-glutamyl carboxylase that converts glutamic acid (Glu) into $\gamma$-carboxyglutamic acid (Gla) residues ${ }^{2}$. This conversion is critical for the activation of MGP. Additionally, there are three serine residues that need phosphorylation ${ }^{4,5}$. The exact role of phosphorylation of MGP is still not known, but it is believed to play an important role in the regulation of secretion of the protein ${ }^{3}$. Upon activation, MGP binds calcium-salts with high affinity, thereby affecting the calcification processes. The importance of MGP in the inhibition of calcification is illustrated by studies of MGP knockout mice, who die within two months after birth due to severe arterial calcification and rupture of the aorta ${ }^{1}$.

Chronic kidney disease (CKD) patients have an extremely high risk for developing vascular disease ${ }^{4}$. VC, manifested both as medial and intimal calcification with distinct pathologies, is a common risk factor in $\mathrm{CKD}^{5}$. Additionally, vitamin $\mathrm{K}$ deficiency is frequently encountered in $\mathrm{CKD}$, which is associated with increased plasma levels of dephosphorylated uncarboxylated MGP (dp-ucMGP) plasma levels ${ }^{6,7}$. Furthermore, increased plasma dp-ucMGP levels associate with $\mathrm{VC}^{8,9}$, cardiovascular morbidity-mortality ${ }^{10}$ and aortic valve calcification ${ }^{11}$. However, the connection between local vessel wall expression of MGP (transcription), production (tissue ucMGP) and excretion (plasma dp-ucMGP) is not well explored in CKD. In addition, the vasculature is exposed to an toxic uremic milieu, which has been shown to affect carboxylase activity causing vascular vitamin $\mathrm{K}$ deficiency and increased VC ${ }^{12}$. Additionally, uremia promote VC in a rat model ${ }^{13}$ and induced bone-specific proteins in cultured VSMCs ${ }^{14}$.

The use of dp-ucMGP has clinical potential as a prognostic biomarker of $\mathrm{VC}^{15}$ and might provide complementary information to traditional cardiovascular disease (CVD) risk factors ${ }^{16}$. Furthermore, carboxylated MGP bind, via the negative charge, to active calcification. The inactive form of MGP (dp-ucMGP) is set free in the circulation because of lack of negative charge to bind to calcium crystals and, thus, might be used as biomarker to identify high-risk CVD patients, allowing early intervention ${ }^{17}$. Furthermore, although single nucleotide polymorphisms (SNP) of MGP have been associated with outcomes in diabetes and $\mathrm{CKD}$, its association to $\mathrm{VC}$ remains obscure. MGP SNP analysis could help us understand the complex nature of MGP expression and regulation. Other VKDPs, such as osteocalcin (OC) share the carboxylation step to become activated. Similar to MGP, uncarboxylated OC (ucOC) is increased in vitamin $\mathrm{K}$ deficiency ${ }^{18}$ and has been reported to have a role in the development of $\mathrm{VC}{ }^{19}$.

We investigated the potential association between MGP and VC in CKD stage 5 patients undergoing living donor renal transplantation (RTx). We link MGP genetics (SNPs), transcription (mRNA) and protein data (immunohistochemistry and plasma levels) to clinical vascular phenotype (CAC and histology). 
Chapter 6

\section{Methods}

Patients and study design

Adult ESRD patients undergoing living donor (LD) kidney transplantation (tx) at the Department of Transplantation Surgery at Karolinska University Hospital between March 2009 and October 2016 were invited to participate in the study. The etiologies of CKD were chronic glomerulonephritis $(n=52)$, hypertension and renovascular disease $(n=8)$, diabetic nephropathy $(n=9)$ and others or unknown causes $(n=72)$. The cohort included CKD5 non-dialysis (ND) patients $(n=51)$, prevalent peritoneal dialysis (PD) patients $(n=39)$ and prevalent hemodialysis (HD) patients $(n=51)$. PD patients were treated (median vintage time 11.4 months) with different combinations of biocompatible glucose-based or amino acid-based, or, for the long dwell, icodextrin-based solutions. HD-patients were treated by conventional maintenance HD or other dialytic techniques such as hemodiafiltration (median vintage time 14.4 months). Sixteen (11\%) out of 141 patients had diabetes. Twenty-three (15\%) of the patients had previously been diagnosed with cerebrovascular, cardiovascular, and/or peripheral vascular disease (grouped as CVD). We measured circulating plasma dp-ucMGP in 141 CKD5 patients including 51 non-dialyzed (CKD5-ND) and 90 CKD5 patients treated by either PD $(n=39)$ or HD $(n=51)$. Patient characteristics are shown in Table 1 and a flow chart of patient inclusion is displayed in supplemental figure 1. Age ranged from 19 to 75 years and patients were recruited from March 2009 to October 2016.

Exclusion criterion was unwillingness to participate. Informed consent was obtained from each patient. The Ethics Committee of the Karolinska Institutet approved study protocols. The studies were conducted in adherence to the Declaration of Helsinki."

\section{Biochemical assessments}

Prior to the LD-RTx, fasting blood samples were drawn and stored in $-80^{\circ} \mathrm{C}$. Biochemical analyses of plasma cholesterol, triglycerides, HDL-cholesterol, hemoglobin, creatinine, calcium, phosphate, albumin (coefficient of variation, CV, 3$4 \%$ ), were performed at the Clinical Chemical Laboratory of Karolinska University Hospital, Stockholm, Sweden. LDL was calculated using the Friedewald formula: [(total cholesterol) - (high-density lipoprotein cholesterol) - (triglycerides/5)].

\section{Plasma dp-ucMGP}

Plasma dp-ucMGP levels were determined using the commercially available IVD CE-marked chemiluminescent InaKtif MGP assay on the IDS-iSYS system (IDS, Boldon, UK). In brief, patient samples and internal calibrators were incubated with magnetic particles coated with murine monoclonal antibodies against dp-MGP, acridinium-labelled murine monoclonal antibodies against ucMGP, and an assay buffer. The magnetic particles were captured using a magnet and washed to remove any unbound analyte. Trigger reagents were added; the resulting light emitted by the acridinium label was directly proportional to the level of dp-ucMGP in the sample. The within-run and total variations of this assay were $0.8-6.2 \%$ and $3.0-8.2 \%$, respectively. The assay measuring range was between 300 and $12,000 \mathrm{pmol} / \mathrm{L}$ and was linear up to $11,651 \mathrm{pmol} / \mathrm{L}$. Assays were performed in a single run by Coagulation Profile BV, department of Biochemistry, Maastricht, the Netherlands. 


\section{Vascular scoring by histology}

Inferior epigastric arteries $(\mathrm{n}=118)$ were collected from patients within $20 \mathrm{~min}$ from the start of kidney transplantation procedure. After fixation (4\% phosphate buffered formalin) and paraffin embedding, 1-2 $\mu \mathrm{m}$ thick tissue sections were stained with hematoxylin, eosin and von Kossa method before evaluation by experienced pathologists. The extent of medial calcification was assessed by a pathologist in vascular biopsies ${ }^{38}$ and graded as 0 to 3 . Patients graded as 0 and 1 represented no/ minimal vascular calcification, and those graded 2 and 3 represented moderate/ extensive vascular calcification.

\section{ucMGP immunohistochemical staining}

After deparaffinization and rehydration of inferior epigastric arteries $(n=20)$, immunostaining for ucMGP was performed as described previously ${ }^{35}$, using a monoclonal MGP antibody directed against uncarboxylated MGP (1mg/mL, 1:400 diluted) and a goat anti-mouse HRP (60 minutes at room temperature; Dako, Golstrup, Denmark) secondary antibody. Detection was performed using Novared stain (Vector Labs, Burlingame, CA), yielding a red color. The degree of ucMGP staining was measured semi-quantitatively and assessed by independent pathologists and graded as 0 to 3. Patients graded as 0 and 1 represented zero to minimal ucMGP staining, and patients graded 2 and 3 represented moderate to extensive ucMGP positivity.

\section{MGP expression}

Inferior epigastric artery samples collected at LD-RTx $(n=41)$ were incubated overnight in AllProtect Tissue Reagent (Qiagen, Hilden, Germany) and subsequently stored at $-70^{\circ} \mathrm{C}$. RNA was isolated from arteries using TRIzol Reagent and $M G P$ mRNA levels were analysed with TaqMan chemistry (Thermo Fisher Scientific, Waltham, MA USA) as previously described ${ }^{36}$.

\section{Genotyping of MGP rs4236, rs 1800801 and rs 1800802}

A subset of 117 CKD5 patients with available peripheral blood samples and 389 controls (anonymous blood donors recruited in Stockholm municipality) underwent genotyping. Genomic DNA was extracted following standard procedures at the Karolinska Biobank. Genotypes for rs4236, rs1800801 and rs1800802 polymorphisms were determined with PCR-based allelic discrimination using TaqMan SNP Genotyping Assays, QuantStudio 5 System (Thermo Fisher Scientific, Waltham, MA, USA) and TaqMan Genotyper Software according to manufacturer's protocols.

\section{CAC score}

Cardiac computed tomography (CT) scans were performed using a 64-channel detector scanner (Lightspeed VCT; General Electric (GE) Healthcare, Milwaukee, WI). $\mathrm{CAC}$ scores were expressed in Agatston units ${ }^{37}$, the protocol and measurements as described previously in detail ${ }^{38}$. Total CAC score was calculated as the sum of CAC scores in the left main artery, the left anterior descending artery, the left circumflex artery, and the right coronary artery. 
Chapter 6

\section{Statistical analyses}

Continuous data are expressed as median (10th to 90th percentile) and nominal or ordinal data as percentage. Statistical significance was set at the level of $\mathrm{P}<0.05$. Comparisons between groups were assessed with the non-parametric Wilcoxon test/ Kruskal-Wallis ANOVA-test for continuous variables and Chi-square test for nominal variables. Non-parametric Spearman rank correlation analysis was used to determine associations between variables. We performed multiple imputation of missing values for multivariate linear regression and multivariate logistic regression using the function PROC MI, with all variables in the covariate section used to produce the values for imputation. The original $n$ for each variable is given throughout. The results for each imputation were generated using PROC REGRESS and LOGISTIC, and then combined using PROC MIANALYZE. We used 20 imputed datasets for this study to ensure that our effect estimates were not overly inaccurate due to Monte Carlo variability. Multivariate linear regression analyses of dp-ucMGP were used and results were shown as standardized $\beta$ regression coefficients. We performed multinomial logistic regression analysis to examine factors associated for determinants of vascular calcification $0-1$ score vs 2 -3. Statistical analyses were performed using statistical software SAS version 9.4 (SAS Campus Drive, Cary, NC, USA) and Stata 15.1 (Stata Corporation, College Station, TX, USA) ${ }^{38}$. Figures were created using GraphPad Prism version 8.3.1 for Windows, GraphPad Software, San Diego, California USA, www.graphpad.com.

\section{Results}

\section{Clinical and biochemical characteristics}

Demographic and clinical characteristics are shown in Table 1 and supplemental Table S1. Patients in the highest tertile of dp-ucMGP (>1491 pM) levels were older and had higher CVD prevalence (25\%). Moreover, serum creatinine was higher in the highest tertile of dp-ucMGP compared to the other two tertiles $(782 \mu \mathrm{mol} / \mathrm{L})$. Additionally, gluOC $(37.8 \mathrm{ng} / \mathrm{mL})$, mid-OC $(90.5 \mathrm{ng} / \mathrm{mL})$ and Osteoprotegerin $(7.6 \mathrm{pg} / \mathrm{mL})$ were also elevated in the highest tertile of dp-ucMGP.

Table 1. Baseline clinical and biochemical characteristics in 141 CKD 5 patients in relation to tertiles of dpucMGP.

\begin{tabular}{|c|c|c|c|c|c|}
\hline & All $(n=141)$ & $1^{\text {st }}$ tertile $(n=46)$ & $2^{n d}$ tertile $(n=47)$ & $3^{\text {rd }}$ tertile $(n=48)$ & $p$-value \\
\hline Age (years) & $47(24,63)$ & $43(22,63)$ & $49(23,62)$ & $50(32,68)$ & 0.008 \\
\hline Males, (\%) & 70 & 65 & 68 & 75 & 0.57 \\
\hline Diabetes mellitus, (\%) & 11 & 7 & 11 & 17 & 0.29 \\
\hline Cardiovascular disease, (\%) & 16 & 7 & 15 & 25 & 0.04 \\
\hline BMI, $\left(\mathrm{kg} / \mathrm{m}^{2}\right)^{*}$ & $24.5(20.8,29.9)$ & $24.0(19.8,29.1)$ & $23.5(20.8,30.4)$ & $25.1(21.8,30.2)$ & 0.17 \\
\hline Systolic BP, (mmHg)* & $140(117,169)$ & $140(116,162)$ & $140(116,167)$ & $144(121,181)$ & 0.29 \\
\hline Diastolic BP, $(\mathrm{mmHg})^{*}$ & $84(69,96)$ & $82(68,92)$ & $82(72,100)$ & $86(67,99)$ & 0.33 \\
\hline Hemoglobin, (g/L)‡ & $114(99,132)$ & $111(98,129)$ & $118(99,135)$ & $113(99,132)$ & 0.09 \\
\hline $\mathrm{HbA} 1 \mathrm{c},(\mathrm{mmol} / \mathrm{mol}) \dagger$ & $33(23,40)$ & $35(26,40)$ & $32(25,40)$ & $32(21,41)$ & 0.06 \\
\hline Triglycerides, (mmol/L) & $1.3(0.7,2.4)$ & $1.2(0.6,2.2)$ & $1.2(0.7,2.6)$ & $1.4(0.7,2.6)$ & 0.58 \\
\hline Total cholesterol, (mmol/L) & $4.5(3.1,6.2)$ & $4.3(3.0,6.1)$ & $4.6(3.2,6.0)$ & $4.5(3.0,6.4)$ & 0.64 \\
\hline HDL cholesterol, (mmol/L) & $1.4(0.9,2.1)$ & $1.4(1.0,2.1)$ & $1.4(0.9,2.0)$ & $1.3(0.8,2.2)$ & 0.37 \\
\hline Serum Creatinine, ( $\mu \mathrm{mol} / \mathrm{L})$ & $723(488,1022)$ & $622(388,985)$ & $743(517,1018)$ & $782(531,1135)$ & 0.01 \\
\hline Serum Albumin $(\mathrm{g} / \mathrm{L})^{*}$ & $35(29,40)$ & $35(30,42)$ & $35(29,40)$ & $36(29,41)$ & 0.80 \\
\hline
\end{tabular}


MGP is an independent predictor of vascular calcification in CKD

\begin{tabular}{|c|c|c|c|c|c|}
\hline Uric Acid, $(\mu \mathrm{mol} / \mathrm{L}) \bullet$ & $369(254,522)$ & $413(285,557)$ & $350(239,510)$ & $357(247,469)$ & 0.02 \\
\hline PEW (SGA>1), n (\%)** & 25 & 31 & 26 & 18 & 0.33 \\
\hline \multicolumn{6}{|c|}{ Vascular calcification biomarkers } \\
\hline $\begin{array}{l}\text { Coronary artery calcium, } \\
\text { (AU) } \ddagger \ddagger\end{array}$ & $8(0,1170)$ & $0(0,236)$ & $8(0,1392)$ & $34(0,1948)$ & 0.002 \\
\hline Calcium, (mmol/L)* & $2.3(2.0,2.5)$ & $2.3(2.1,2.4)$ & $2.3(2.0,2.6)$ & $2.3(1.9,2.6)$ & 0.67 \\
\hline Phosphate, $(\mathrm{mmol} / \mathrm{L})^{*}$ & $1.6(1.0,2.4)$ & $1.7(1.0,2.2)$ & $1.6(1.1,2.5)$ & $1.7(0.9,2.5)$ & 0.96 \\
\hline Glu-OC, $(\mathrm{ng} / \mathrm{mL})^{* *}$ & $14.0(1.8,87.6)$ & $7.5(1.6,33.7)$ & $15.5(1.7,122.6)$ & $37.8(2.3,99.2)$ & 0.001 \\
\hline Gla-OC, $(\mathrm{ng} / \mathrm{mL})^{* *}$ & $33.2(11.7,97.6)$ & $26.7(11.0,73.5)$ & $33.2(11.7,138.0)$ & $42.9(9.7,102.2)$ & 0.23 \\
\hline Mid-OC, $(\mathrm{ng} / \mathrm{mL})$ ๆๆ & $59.5(13.5,259.5)$ & $37.9(11.6,178.6)$ & $61.8(11.3,218.6)$ & $90.5(17.7,395.8)$ & 0.02 \\
\hline Osteoprotegerin, (pg/ml) & $6.1(3.8,12.0)$ & $5.6(2.8,10.1)$ & $5.9(3.9,11.1)$ & $7.6(4.2,14.0)$ & 0.002 \\
\hline
\end{tabular}

Data presented as median (10th - 90th percentile) or percentage

Abbreviations: CKD, chronic kidney disease; dp-ucMGP, dephosphorylated uncarboxylated Matrix Gla protein; BMI (body mass index); BP, blood pressure; HbAlc, Hemoglobin A1c; HDL, high-density lipoprotein; PEW, protein energy wasting; SGA, subjective global assessment; AU, Agatson unit; Glu-OC, undercarboxylated osteocalcin; Gla-OC, carboxylated osteocalcin; mid-OC, n-mid osteocalcin * $n=139$ : **n=

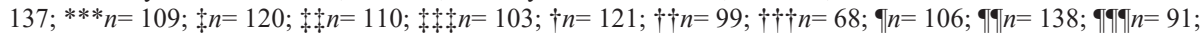
$\bullet n=128 ; \bullet n=140 ; \cdots n=114$

\section{dp-ucMGP levels were associated with increased CAC and medial calcification score}

Patients in the $3^{\text {rd }}$ tertile showed higher CAC score compared to the other two tertiles ranging from a CAC score with a median $\left(10^{\text {th }}-90^{\text {th }}\right.$ percentile $)$ of $0(0,236)$ in the $1^{\text {st }} 8(8,1392)$ in the $2^{\text {nd }}$ and $34(0,1948)$ in the $3^{\text {rd }} d p$-ucMGP tertile $(p=0.002)$ (Figure 1a). Medial VC (scored by pathologists, ranging from 0 - no, 1 - mild, 2 moderate, to 3 - severe calcification) was associated with an increase in dp-ucMGP levels (median 1193 in group 0-1 and 1458 in group 2-3) $(\mathrm{p}<0.05)$ (Figure 1b).

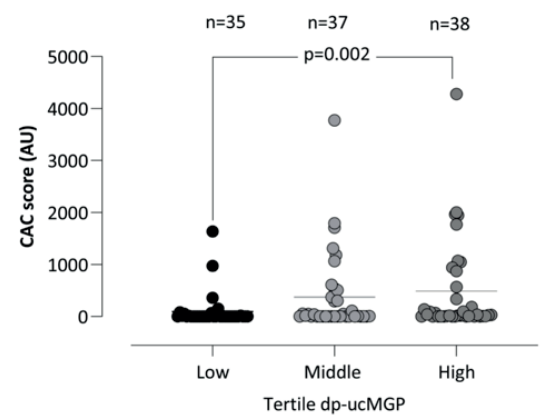

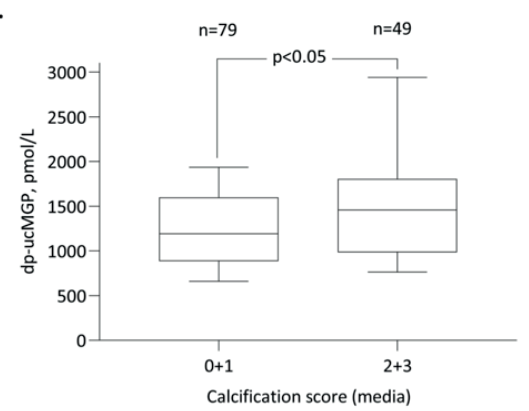

Figure 1. dp-ucMGP levels associate to CAC score and vascular media calcification score. (a) CAC score measured in 110 CKD5 patients in relation to tertiles of dp-ucMGP levels. (b) dp-ucMGP levels of 128 CKD5 patients displayed against low (score $0+1)$ and high (score $2+3$ ) media VC.

\section{Calcification of the vascular media co-localized with ucMGP}

Patients that had a medial VC score of 0 showed no staining for ucMGP (Figure 2a). On the contrary, patients that had a medial VC score of 3 showed a strong staining of ucMGP around calcified areas (Figure 2b). Calcified areas are accompanied by a loss of structure and show no cellular components, the arrows indicate calcified areas that stain highly positive for ucMGP (Figure $2 b$ ). 
a.
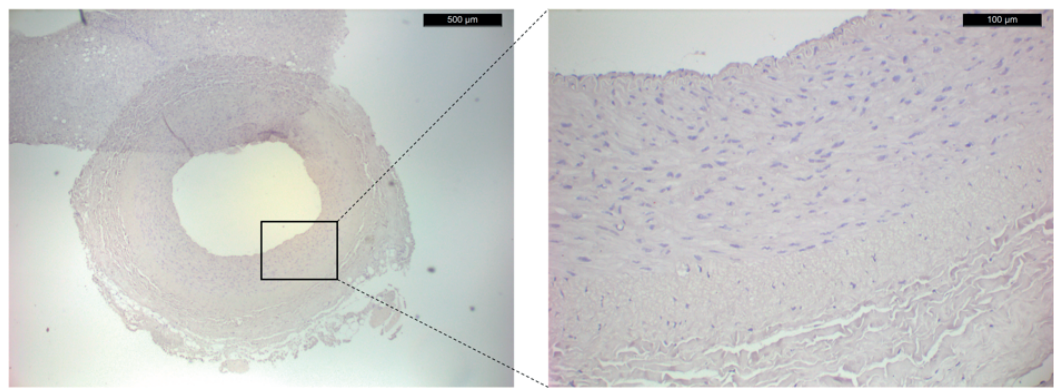

b.
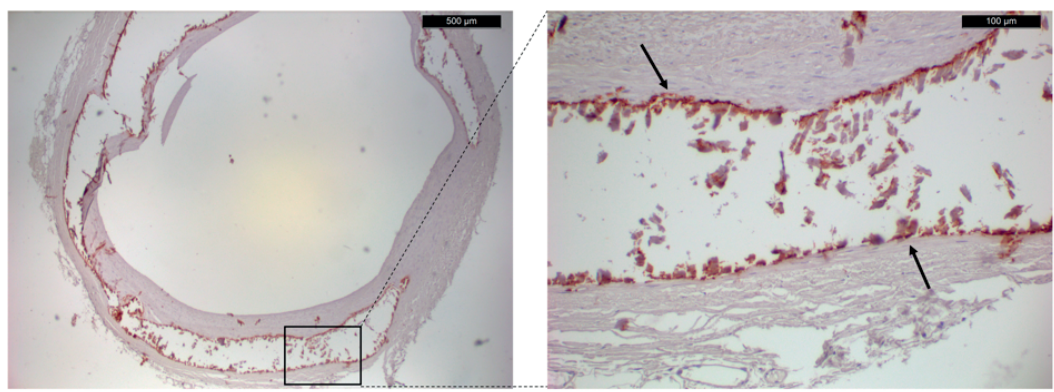

Figure 2. Immunohistochemical staining of ucMGP in epigastric artery from CKD5 patients. (a) shows ucMGP staining of epigastric artery of a patient with a medial VC score of 0 (left, 4x; right, 20x). (b) shows ucMGP staining of an epigastric artery with a medial VC score of 3 (left, 4x; right, 20x).

\section{Multivariate analysis of factors associated with dp-ucMGP}

In multivariate linear regression analysis of determinants of dp-ucMGP were bone markers i.e. 1 -SD increase of ucOC (est $0.48 ; p=0.001$ ) and OPG (est $0.28 ; p=0.02$ ) after adjusting for 1-SD increase of age and albumin (Table 2).

Table 2. Multiple linear regression models for 1-standard deviation increase of plasma dp-ucMGP in 141 CKD 5 patients.

\begin{tabular}{l|c} 
& Model (coefficient +SE, p-value) \\
\hline 1-SD increase of age, years & $0.05+0.12(0.69)$ \\
$1-S D$ increase of uCOC, $\mathrm{ng} / \mathrm{ml}$ & $0.48+0.14(0.001)$ \\
$1-S D$ increase of albumin, $\mathrm{g} / \mathrm{L}$ & $0.13+0.12(0.27)$ \\
$1-S D$ increase of Mid-OC, $\mathrm{ng} / \mathrm{ml}$ & $-0.21+0.15(0.16)$ \\
1-SD increase of osteoprotegerin, $\mathrm{pg} / \mathrm{ml}$ & $0.28+0.12(0.02)$ \\
\hline
\end{tabular}

Abbreviations; 1-SD, one standard deviation; dp-ucMGP, dephosphorylated uncarboxylated Matrix Gla protein; CKD, chronic kidney disease; SE, standard error; ucOC, undercarboxylated osteocalcin; mid-OC, nmid osteocalcin

\section{Multivariate analysis of factors associated with medial calcification}

In a multivariate logistic regression analysis of factors associated with high medial VC (Table 3) (VC score media $(0-1)=$ reference), high medial VC was associated with age $(p=0.006)$, sex $(p=0.001)$, dp-ucMGP $(p=0.04)$ and DM $(p=0.008)$ after adjusting for hsCRP and CAC score $(\mathrm{p}=0.059)$ (Table 3$)$. 
MGP is an independent predictor of vascular calcification in CKD

Table 3. Multinominal logistic regression analysis of factors associated with high calcification score media $(2+3)\left(n=122\right.$, pseudo $\left.r^{2}=0.22\right)$. Patients with calcification score media $0+1$ served as the reference.

\begin{tabular}{lcc}
\cline { 2 - 3 } & Odds ratio (95\%Cl) & p-value \\
\hline 1-SD increase of age, years & $2.3(1.3-4.1)$ & 0.006 \\
1-SD increase of dp-ucMGP, pmol/L & $1.6(1.0-2.5)$ & 0.04 \\
Gender (female, male) & $7.3(2.2-25.1)$ & 0.001 \\
1-SD increase of hsCRP, mg/L & $1.3(0.4-1.3)$ & 0.31 \\
Diabetes mellitus (yes, no) & $26.0(2.3-293.2)$ & 0.008 \\
1-SD increase of CAC-score, AU & $1.2(0.5-2.9)$ & 0.59
\end{tabular}

Abbreviations: 95\%CI, 95\% confidence interval; 1- SD, one standard deviation; dp-ucMGP, dephosphorylated uncarboxylated Matrix Gla protein; hsCRP, high-sensitivity C-reactive protein; CAC, coronary artery calcium; $\mathrm{AU}$, Agatston unit

\section{High arterial $M G P$ mRNA expression associates with high CAC}

We divided patients according to median of MGP mRNA expression. High expression of MGP $(n=41)$ showed higher CAC score with a median $\left(10^{\text {th }}-90^{\text {th }}\right.$ percentile) of 16 (0 - 1804), compared to the low MGP expression group with a median $\left(10^{\text {th }}-90^{\text {th }}\right.$ percentile $)$ of $0(0-255)(\mathrm{p}=0.036)$ (Figure $\left.3 \mathrm{a}\right)$. Additionally, high expression of MGP showed higher dp-ucMGP plasma levels, with a median $\left(10^{\text {th }}-90^{\text {th }}\right.$ percentile $)$ of 1459 (711 - 5313), compared to low MGP expression with a median $\left(10^{\text {th }}-90^{\text {th }}\right.$ percentile) of 1100 (760 - 1653) ( $\mathrm{p}=0.034)$ (Figure 3b). In a Spearman's rank (rho) analyses, MGP expression was significantly correlated with CAC score $(\mathrm{Rho}=0.39)$ and medial calcification score $(\mathrm{Rho}=0.37)$. After adjusting for age and sex in a multivariate linear regression analysis, these significant correlations were lost.

a.

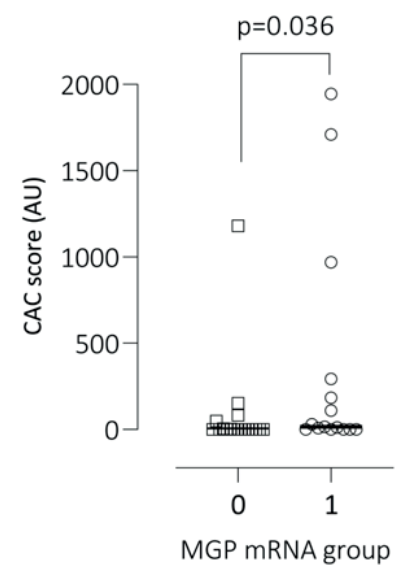

b.

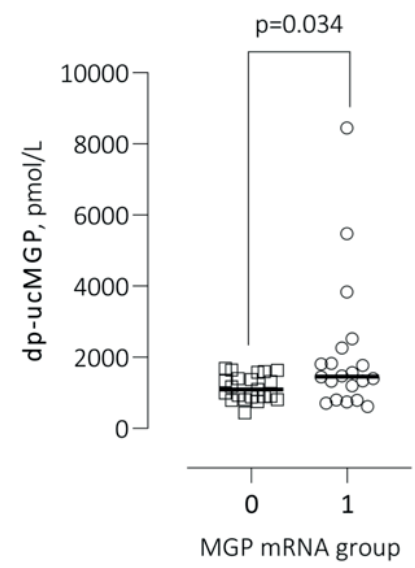

Figure 3. Association between MGP expression and CAC and dp-ucMGP. (a) MGP expression measured in 41 CKD5 patients in relation to CAC score. (b) MGP expression measured in 41 CKD5D patients in relation to dp-ucMGP plasma levels. 


\section{MGP polymorphisms - relation to circulating dp-ucMGP, arterial $M G P$ mRNA and $\mathrm{VC}$}

Distributions of MGP rs4236, rs1800801, rs1800802 polymorphisms in CKD5 patients and controls are presented in Supplemental Table S2. The genotype frequencies of the rs4236 and rs1800801, but not rs1800802, differed significantly between patients and controls: in patients, the $\mathrm{rs} 4236 \mathrm{~T} / \mathrm{T}$ and $\mathrm{rs} 1800801 \mathrm{C} / \mathrm{C}$ genotypes were more common (44\% vs. $39 \%$ and $53 \%$ vs. $44 \%$, respectively), whereas $\mathrm{rs} 4236 \mathrm{C} / \mathrm{C}$ and rs $1800801 \mathrm{~T} / \mathrm{T}$ were less frequent (both 6\% vs. 15\%) compared to controls. A selection of patient characteristics was investigated in relation to MGP genotypes (Supplemental Table S3). None of the MGP SNPs were associated with age, sex, DM or dp-ucMGP levels. Neither the rs1800801 nor the rs1800802 SNPs associated with MGP mRNA levels but when dividing patients according to rs4236 T-homozygotes vs. C-allele carriers, $M G P$ mRNA levels were found to be higher in patients homozygous for the $\mathrm{T}$ allele compared to patients with CC and CT genotype ( 1.3 vs. $0.9 \mathrm{RQ}, \mathrm{p}=0.03)$. Whereas patient's CAC scores were not associated with MGP genotypes, media VC score differed significantly according to rs 1800801 genotype, with $50 \%$ of $\mathrm{C}$ homozygotes having a moderate to extensive calcification (i.e. score 2 or 3 ) compared to $24 \%$ and $14 \%$, respectively, in patients with $\mathrm{C} / \mathrm{T}$ and $\mathrm{T} / \mathrm{T}$ genotypes $(\mathrm{p}=0.01)$ (Supplemental Table S3). When comparing $\mathrm{C}$-allele homozygotes to T-allele carriers in relation to either none/minimal calcification (score $0-1$ ) or moderate/extensive calcification (score 2-3) the difference became even more significant ( $50 \%$ vs. $22 \%, n=109 ; \mathrm{p}=0.005)$. Similarly, patients that were homozygous for the rs4236 T-allele had more often moderateextensive medial VC than patients carrying a C-allele (49\% vs. 27\%, $\mathrm{p}=0.02)$.

\section{Discussion}

We examined the role of MGP as a potential biomarker for the extent of VC in CKD5 patients and confirmed than an increase in plasma dp-ucMGP levels was significantly associated with an increase in both CAC score and medial arterial VC. CKD patients are predisposed to $\mathrm{VC}^{20}$, an independent risk factor for CVD morbidity and mortality ${ }^{4,21}$. CAC score determines the extent of CAC and is correlated to $\mathrm{VC}$ at other places in the arterial tree ${ }^{22-24}$. However, as CT of the heart involves radiation and its value to determine microcalcifications is limited, circulating biomarkers that reflect early $\mathrm{VC}$ would be cheaper, safer and less invasive. Additionally, to interfere with VC progression, biomarkers that predict initiation phases of VC, such as MGP are of value in prediction and to follow treatment of VC.

MGP is a potent inhibitor of VC produced by VSMCs ${ }^{25}$, which are pivotal in maintaining MGP homeostasis, as they are involved in both the production and activation of MGP, both of which are important for the subsequent inhibition of VC ${ }^{21,25}$. Since MGP is an active inhibitor of VC it could be a potential useful biomarker. Indeed, circulating plasma levels of dp-ucMGP correlate with amount of $\mathrm{VC}^{22,23}$ and arterial stiffness ${ }^{26}$. In accordance, we report that patients with the highest dp-ucMGP levels had a higher CAC score and more extensive medial VC.

CKD patients suffer from vitamin K deficiency and are prone to develop $\mathrm{VC}^{24}$. Since vitamin K is necessary for the activation (carboxylation) of MGP in the vessel wall, MGP will be produced in the inactive form when levels of vitamin $\mathrm{K}$ are low ${ }^{25}$. We show that next to circulating levels of dp-ucMGP, highly calcified epigastric arteries also show co-localization with ucMGP. This data supports the hypothesis that circulating 
dp-ucMGP reflects local tissue levels of ucMGP which might be caused by a vascular vitamin $\mathrm{K}$ deficiency, a risk factor for VC. Although vitamin K intake or plasma levels were not measured in the present study, our findings indirectly imply that vitamin $\mathrm{K}$ status is low since also ucOC was significantly increased ${ }^{27}$. After correction for age and albumin, dp-ucMGP remained significant for bone markers. Moreover, after correction for CAC score and inflammation, dp-ucMGP remained significant for medial calcification score.

In this study, high MGP expression was associated with a CAC score and increased medial calcification (not significant) scored by pathologists. High expression of MGP infers to a high local production of MGP. However, nothing is known about the activation state of this locally produced MGP. As CKD patients are vitamin K deficient ${ }^{24}$, we hypothesize that most of the MGP is present as inactive MGP. This accord with our results showing a strong association between MGP expression and circulating dpucMGP. In vitro, low expression of MGP was associated with calcification via upregulation of BMP-2 ${ }^{28}$. Additionally, under specific pro-calcific conditions, such as a high calcium concentration, calcification is increased whereas MGP expression is decreased ${ }^{29}$. This is contradictory to our results but might be explained by the carboxylation efficiency of MGP. Total MGP in the plasma is the sum of both active (cMGP) and inactive (ucMGP) MGP; thus, high levels of dp-ucMGP do not reflect total level of MGP that is expressed in the tissues. The importance of MGP as inhibitor of VC is demonstrated by MGP ko studies in mice that showed increased soft tissue calcification ${ }^{30}$. Moreover, $\mathrm{MGP}^{-/ /}$mice express higher levels of osteogenic proteins, such as osteopontin, OC and Cbfa ${ }^{31}$. Due to the complex nature of MGP, further studies need to examine the precise relation between local vasculature MGP expression and circulating MGP.

Besides measurement of plasma dp-ucMGP, we genotyped a subgroup of patients for three well-known SNPs (rs4236, rs1800801 and rs1800802) in the MGP gene. In comparison to healthy controls, our subgroup of CKD patients differed in respect to two of the SNPs, rs4236 and rs1800801, with patients displaying clearly lower frequencies of the minor alleles $\mathrm{C}$ and $\mathrm{T}$, respectively. As none of the SNPs were associated with plasma dp-ucMGP levels, the biological impact is unclear. However, because the MGP rs1800801 T-allele is associated with lower medial VC score, this allele may be protective. The literature is, however, conflicting regarding the potential protective effect of the T-allele on vascular disease. A meta-analysis by Sheng et al ${ }^{32}$ showed that the MGP T-allele was associated with a higher risk for VC whereas Taylor et al. ${ }^{33}$ found no association between the MGP rs1800801 and CAC score. An explanation for the conflicting evidence might be the differences in ethnic composition between the study populations and limited power.

The present results should be considered in light of some strengths and limitations. The relatively low sample size restricts power of the multivariate analyses and information retrieved from the MGP gene variants. Moreover, the observational nature of the study restricts causal conclusions. Furthermore, results obtained in this study are retrieved from different patient cohorts which means that our study population is heterogenous, for example displaying a population aged from 19-75. Since dp-ucMGP only represents a minor fraction of the total ucMGP content it should be interpreted with care ${ }^{17}$. CAC determination by cardiac CT cannot distinguish between medial or intimal calcification. However, we did find a strong association between medial $\mathrm{VC}$ in the 


\section{Chapter 6}

epigastric artery and CAC. Finally, expression levels of MGP were only measured in limited number of patients and further studies are needed to examine the role of MGP expression in VC. For the MGP gene variants we need to perform additional in vitro and in vivo experiments to assess its causal role.

As increased dp-ucMGP levels associate with increased CAC and medial VC our results support that dp-ucMGP is an independent predictor of $\mathrm{VC}$ and a risk factor for arterial stiffness and cardiovascular mortality ${ }^{34}$. Alterations in plasma dp-ucMGP correlate with local tissue expression of ucMGP around areas of VC, low general MGP expression and genomic SNP analysis. Although our data indicates a predictive value of MGP as a biomarker for VC, further studies need to confirm whether these finding translate into CVD morbidity and mortality. Additionally, discovering the precise regulation of MGP in VC may provide novel therapeutic approaches with a potential role for vitamin $\mathrm{K}$ supplementation. 


\section{References}

1. Luo, G. et al. Spontaneous calcification of arteries and cartilage in mice lacking matrix GLA protein. Nature 386, 78-81 (1997).

2. Price, P. A., Faus, S. A. \& Williamson, M. K. Warfarin Causes Rapid Calcification of the Elastic Lamellae in Rat Arteries and Heart Valves. Arteriosclerosis, Thrombosis, and Vascular Biology 18, 1400-1407 (1998).

3. Wajih, N., Borras, T., Xue, W., Hutson, S. M. \& Wallin, R. Processing and transport of matrix gamma-carboxyglutamic acid protein and bone morphogenetic protein-2 in cultured human vascular smooth muscle cells: evidence for an uptake mechanism for serum fetuin. J. Biol. Chem. 279, 4305243060 (2004).

4. O'Shaughnessy, M. M., Liu, S., Montez-Rath, M. E., Lafayette, R. A. \& Winkelmayer, W. C. Cause of kidney disease and cardiovascular events in a national cohort of US patients with end-stage renal disease on dialysis: a retrospective analysis. Eur. Heart J. 40, 887-898 (2019).

5. Bashir, A. et al. Coronary Artery Calcium Assessment in CKD: Utility in Cardiovascular Disease Risk Assessment and Treatment? Am. J. Kidney Dis. 65, 937-948 (2015).

6. Westenfeld, R. et al. Effect of vitamin K2 supplementation on functional vitamin $\mathrm{K}$ deficiency in hemodialysis patients: a randomized trial. Am. J. Kidney Dis. 59, 186-195 (2012).

7. Dalmeijer, G. W. et al. Matrix Gla Protein Species and Risk of Cardiovascular Events in Type 2 Diabetic Patients. Diabetes Care 36, 3766-3771 (2013).

8. Delanaye, P. et al. Dephosphorylated-uncarboxylated Matrix Gla protein concentration is predictive of vitamin $\mathrm{K}$ status and is correlated with vascular calcification in a cohort of hemodialysis patients. BMC Nephrol 15, 145 (2014).

9. Schurgers, L. J. et al. The circulating inactive form of matrix gla protein is a surrogate marker for vascular calcification in chronic kidney disease: a preliminary report. Clin J Am Soc Nephrol 5, 568575 (2010).

10. Ueland, T. et al. Circulating levels of non-phosphorylated undercarboxylated matrix Gla protein are associated with disease severity in patients with chronic heart failure. Clinical Science 121, 119-127 (2011).

11. Brandenburg, V. M. et al. Slower Progress of Aortic Valve Calcification With Vitamin K Supplementation: Results From a Prospective Interventional Proof-of-Concept Study. Circulation 135, 2081-2083 (2017).

12. Kaesler, N. et al. Impaired vitamin $\mathrm{K}$ recycling in uremia is rescued by vitamin $\mathrm{K}$ supplementation. Kidney Int. 86, 286-293 (2014).

13. Opdebeeck, B. et al. Indoxyl Sulfate and p-Cresyl Sulfate Promote Vascular Calcification and Associate with Glucose Intolerance. J. Am. Soc. Nephrol. 30, 751-766 (2019).

14. Muteliefu, G., Enomoto, A., Jiang, P., Takahashi, M. \& Niwa, T. Indoxyl sulphate induces oxidative stress and the expression of osteoblast-specific proteins in vascular smooth muscle cells. Nephrol. Dial. Transplant. 24, 2051-2058 (2009).

15. Schurgers, L. J., Cranenburg, E. C. M. \& Vermeer, C. Matrix Gla-protein: the calcification inhibitor in need of vitamin K. Thromb. Haemost. 100, 593-603 (2008).

16. Dalager, S., Falk, E., Kristensen, I. B. \& Paaske, W. P. Plaque in superficial femoral arteries indicates generalized atherosclerosis and vulnerability to coronary death: an autopsy study. Journal of Vascular Surgery 47, 296-302 (2008).

17. Cranenburg, E. C. M. et al. Characterisation and potential diagnostic value of circulating matrix Gla protein (MGP) species. Thromb. Haemost. 104, 811-822 (2017).

18. Nagata, Y. et al. Increased undercarboxylated osteocalcin/intact osteocalcin ratio in patients undergoing hemodialysis. Osteoporos Int 26, 1053-1061 (2015).

19. Okura, T. et al. Undercarboxylated osteocalcin is a biomarker of carotid calcification in patients with essential hypertension. Kidney Blood Press. Res. 33, 66-71 (2010).

20. Goodman, W. G. et al. Coronary-Artery Calcification in Young Adults with End-Stage Renal Disease Who Are Undergoing Dialysis. http://dx.doi.org/10.1056/NEJM200005183422003 342, 1478-1483 (2009).

21. Block, G. A., Raggi, P., Bellasi, A., Kooienga, L. \& Spiegel, D. M. Mortality effect of coronary calcification and phosphate binder choice in incident hemodialysis patients. Kidney Int. 71, 438-441 (2007).

22. Chatrou, M. L. L., Winckers, K., Hackeng, T. M., Reutelingsperger, C. P. \& Schurgers, L. J. Vascular calcification: The price to pay for anticoagulation therapy with vitamin K-antagonists. YBLRE 26, 155-166 (2012). 


\section{Chapter 6}

23. Rennenberg, R. J. M. W. et al. Vascular calcifications as a marker of increased cardiovascular risk: a meta-analysis. Vasc Health Risk Manag 5, 185-197 (2009).

24. Cranenburg, E. C. M. et al. Vitamin K intake and status are low in hemodialysis patients. Kidney Int. 82, 605-610 (2012).

25. Schurgers, L. J., Uitto, J. \& Reutelingsperger, C. P. Vitamin K-dependent carboxylation of matrix Gla-protein: a crucial switch to control ectopic mineralization. Trends Mol Med 19, 217-226 (2013).

26. Puzantian, H. et al. Circulating Dephospho-Uncarboxylated Matrix Gla-Protein Is Associated With Kidney Dysfunction and Arterial Stiffness. Am. J. Hypertens. 31, 988-994 (2018).

27. Lian, J. B. \& Gundberg, C. M. Osteocalcin. Clinical Orthopaedics and Related Research \&NA, 267291 (1988).

28. Chiyoya, M. et al. Matrix Gla protein negatively regulates calcification of human aortic valve interstitial cells isolated from calcified aortic valves. J. Pharmacol. Sci. 136, 257-265 (2018).

29. Wang, Q. et al. High Concentration of Calcium Promotes Mineralization in NRK-52E Cells Via Inhibiting the Expression of Matrix Gla Protein. Urology 119, 161.e1-161.e7 (2018).

30. Lanham, S. A., Cagampang, F. R. \& Oreffo, R. O. C. The influence of a high fat diet on bone and soft tissue formation in Matrix Gla Protein knockout mice. Sci Rep 8, 3635 (2018).

31. Steitz, S. A. et al. Smooth muscle cell phenotypic transition associated with calcification: upregulation of $\mathrm{Cbfa} 1$ and downregulation of smooth muscle lineage markers. Circ. Res. 89, 1147 1154 (2001).

32. Sheng, K. et al. Association of Matrix Gla protein gene (rs1800801, rs1800802, rs4236) polymorphism with vascular calcification and atherosclerotic disease: a meta-analysis. Sci Rep 7, 8713 (2017).

33. Taylor, B. C. et al. Matrix Gla protein and osteopontin genetic associations with coronary artery calcification and bone density: the CARDIA study. Hum. Genet. 116, 525-528 (2005). Zanoli, L. et al. Arterial Stiffness in the Heart Disease of CKD. J. Am. Soc. Nephrol. 30, 918-928 (2019).

35. Schurgers, L. J. et al. Novel conformation-specific antibodies against matrix gammacarboxyglutamic acid (Gla) protein: undercarboxylated matrix Gla protein as marker for vascular calcification. Arteriosclerosis, Thrombosis, and Vascular Biology 25, 1629-1633 (2005).

36. Stenvinkel, P. et al. CDKN2A/p16INK4a expression is associated with vascular progeria in chronic kidney disease. Aging 9, 494-507 (2017).

37. Agatston, A. S. et al. Quantification of coronary artery calcium using ultrafast computed tomography. J. Am. Coll. Cardiol. 15, 827-832 (1990).

38. Qureshi, A. R. et al. Increased circulating sclerostin levels in end-stage renal disease predict biopsyverified vascular medial calcification and coronary artery calcification. Kidney Int. 88, 1356-1364 (2015). 


\section{Supplemental material}

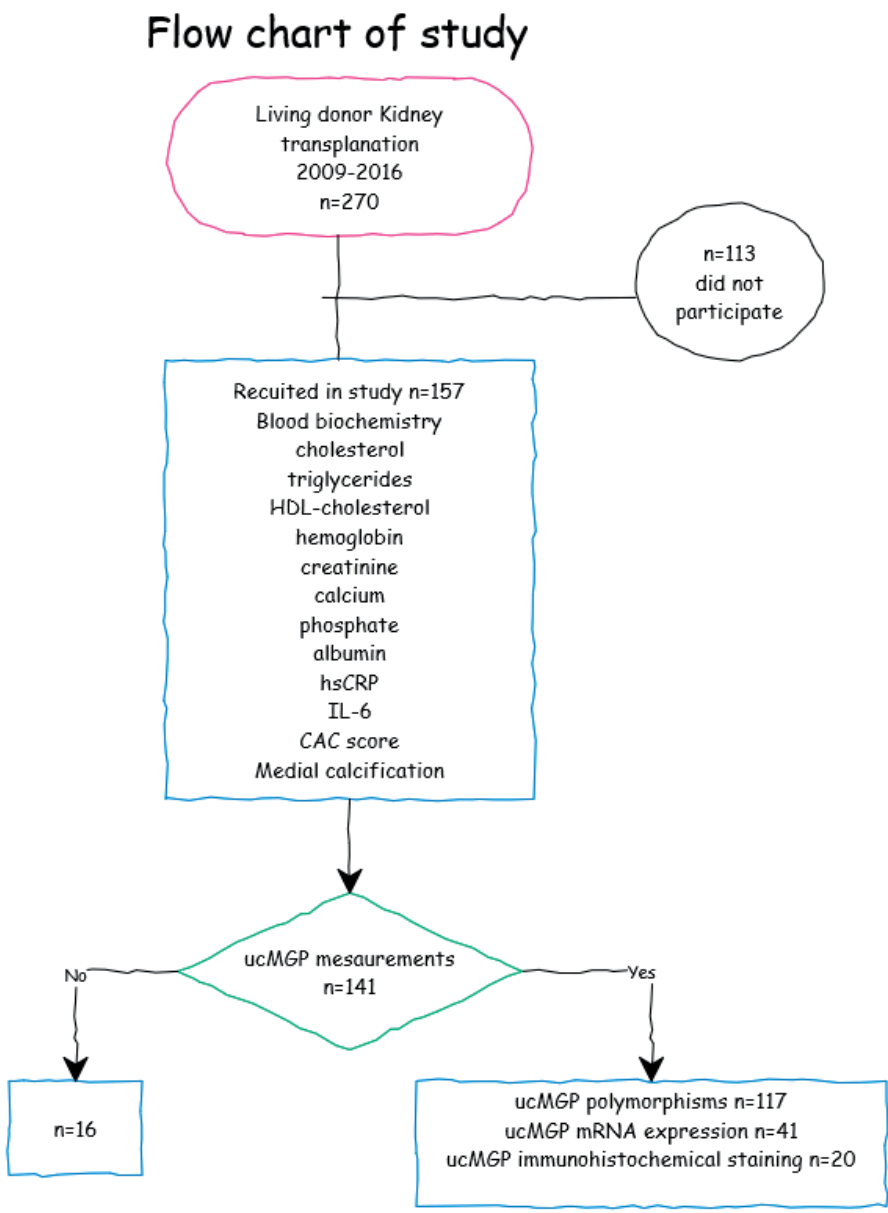

Supplemental Figure S1. Flow chart of patient inclusion. 


\section{Chapter 6}

Supplemental Table S1. Other baseline clinical and biochemical characteristics in 141 CKD 5 patients in relation to tertiles of dp-ucMGP.

$$
\text { All }(n=141) \quad 1^{\text {st }} \text { tertile }(n=46) \quad 2^{\text {nd }} \text { tertile }(n=47) \quad 3^{\text {rd }} \text { tertile }(n=48) \quad p \text {-value }
$$

\begin{tabular}{|c|c|c|c|c|c|}
\hline iPTH, (ng/L) & $261(83,558)$ & $255(81,747)$ & $270(84,502)$ & $290(49,563)$ & 0.72 \\
\hline $\begin{array}{l}1,25-\mathrm{OH} \\
(\mathrm{mmol} / \mathrm{L}) \dagger \dagger\end{array} \quad$ vitamin & $18(9,32)$ & $18(11,35)$ & $18(9,33)$ & $17(7,32)$ & 0.89 \\
\hline $\begin{array}{l}25(\mathrm{OH}) \text { D-vitamin, } \\
(\mathrm{mmol} / \mathrm{L}) \text { ๆ }\end{array}$ & $35(17,72)$ & $33(16,75)$ & $38(12,69)$ & $36(21,73)$ & 0.64 \\
\hline hsCRP, $(\mathrm{mg} / \mathrm{L}) \bullet$ & $0.7(0.2,4.0)$ & $0.5(0.2,2.5)$ & $0.7(0.2,3.9)$ & $1.5(0.3,10.7)$ & $<0.001$ \\
\hline IL-6, $(\mathrm{pg} / \mathrm{mL}) * * *$ & $1.3(0.1,4.1)$ & $0.9(0.01,3.0)$ & $1.6(0.3,3.4)$ & $1.4(0.02,6.6)$ & 0.06 \\
\hline $\mathrm{TNF},(\mathrm{pg} / \mathrm{mL})+t+$ & $10.5(7.6,16.5)$ & $8.9(7.3,12.3)$ & $10.7(7.3,16.1)$ & $12.6(9.1,20.4)$ & $<0.001$ \\
\hline $\mathrm{CTX},(\mathrm{ng} / \mathrm{ml}) \bullet$ & $1.8(0.5,5.1)$ & $1.8(0.4,4.9)$ & $1.8(0.6,5.5)$ & $1.9(0.5,4.4)$ & 0.88 \\
\hline FGF-19, (pg/ml) * & $117(41,331)$ & $133(39,439)$ & $86(35,263)$ & $135(53,343)$ & 0.02 \\
\hline FGF-23, $\dagger \dagger+(\mathrm{pg} / \mathrm{ml})$ & $4055(676,48027)$ & $3668(350,32935)$ & $7581(826,44887)$ & $6391(624,99267)$ & 0.40 \\
\hline Klotho, $(\mathrm{pg} / \mathrm{ml}) \bullet$ & $389(213,669)$ & $366(188,763)$ & $382(212,671)$ & $414(257,585)$ & 0.66 \\
\hline ALP, (U/L) & $65.9(39.8,118.3)$ & $69.7(41.7,118.2)$ & $65.1(37.4,122.9)$ & $65.5(39.4,121.5)$ & 0.95 \\
\hline BALP, (ug/L) & $15,8(8.6,41.5)$ & $16.1(8.9,40.7)$ & $14.7(8.6,41.1)$ & $17.2(8.3,43.4)$ & 0.84 \\
\hline Sclerostin, $(\mathrm{pg} / \mathrm{ml}) * *$ & $432(245,830)$ & $409(215,802)$ & $440(244,807)$ & $431(259,897)$ & 0.55 \\
\hline \multicolumn{6}{|c|}{ Medication } \\
\hline Statins, (\%) & 37 & 30 & 36 & 44 & 0.41 \\
\hline$\beta$-blockers, $(\%)$ & 58 & 44 & 62 & 69 & 0.04 \\
\hline Calcium blocker, $(\%)$ & 51 & 54 & 55 & 44 & 0.46 \\
\hline Phosphate binders, $(\%)$ & 53 & 54 & 53 & 52 & 0.98 \\
\hline
\end{tabular}

Data presented as median (10th - 90th percentile) or percentage 
MGP is an independent predictor of vascular calcification in CKD

Supplemental Table S2. Genotype distributions of $M G P$ polymorphisms rs4236, rs 1800801 and rs 1800802 in 117 CKD5 patients and 389 controls.

\begin{tabular}{|c|c|c|c|c|}
\hline & & CKD5 patients & Controls & P-value* \\
\hline MGP rs4236, n (\%) & $\begin{array}{l}\text { T/T } \\
\text { T/C } \\
\text { C/C } \\
\end{array}$ & $\begin{array}{c}51(44) \\
59(50) \\
7(6) \\
\end{array}$ & $\begin{array}{c}152(39) \\
177(46) \\
60(15) \\
\end{array}$ & 0.03 \\
\hline MGP rs1800801, n (\%) & $\begin{array}{l}\mathrm{C} / \mathrm{C} \\
\mathrm{C} / \mathrm{T} \\
\mathrm{T} / \mathrm{T} \\
\end{array}$ & $\begin{array}{c}62(53) \\
48(41) \\
7(6) \\
\end{array}$ & $\begin{array}{c}171(44) \\
161(41) \\
57(15)\end{array}$ & 0.03 \\
\hline MGP rs1800802, n (\%) & $\begin{array}{l}\mathbf{A} / \mathbf{A} \\
\mathbf{A} / \mathbf{G} \\
\mathbf{G} / \mathbf{G}\end{array}$ & $\begin{array}{c}69(59) \\
42(36) \\
6(5) \\
\end{array}$ & $\begin{array}{c}232(59) \\
145(37) \\
16(4)\end{array}$ & 0.89 \\
\hline
\end{tabular}

*Pearson's Chi-Square test 
Supplemental Table S3. Distribution of patient characteristics in relation to $M G P$ genotypes in 117 CKD5 patients.

\begin{tabular}{|c|c|c|c|c|c|}
\hline \multicolumn{4}{|c|}{$M G P$ genotypes } & \multicolumn{2}{|c|}{ P-value } \\
\hline \multicolumn{6}{|c|}{ rs4236 } \\
\hline & & $\mathbf{T} / \mathbf{T}$ & $\mathbf{T} / \mathbf{C}$ & $\mathrm{C} / \mathrm{C}$ & \\
\hline Age (years) & & $47(24-64)$ & $45(23-62)$ & $39(28-66)$ & 0.58 \\
\hline Males (\%) & & 76 & 63 & 43 & 0.11 \\
\hline DM (\%) & & 10 & 8 & 14 & 0.88 \\
\hline dp-ucMGP (pmol/L) & & $1333(745-2218)$ & $1193(661-2526)$ & $1329(799-1998)$ & 0.65 \\
\hline$M G P$ mRNA $(\mathrm{RQ})^{\neq}$ & & $1.2(0.3-3.4)$ & $0.9(0.4-1.7)$ & $0.61(0.5-0.8)$ & 0.15 \\
\hline CAC score $(\mathbf{A U})^{\#}$ & & $37(0-1729)$ & $1(0-1068)$ & $0(0-12)$ & 0.09 \\
\hline \multirow[t]{4}{*}{ Media VC score, $(\%)^{\square}$} & $\mathbf{0}$ & 11 & 18 & 29 & 0.30 \\
\hline & 1 & 40 & 54 & 57 & \\
\hline & 2 & 31 & 20 & 0 & \\
\hline & 3 & 18 & 9 & 14 & \\
\hline \multicolumn{6}{|c|}{ rs1800801 } \\
\hline & & $\mathrm{C} / \mathrm{C}$ & $\mathbf{C} / \mathbf{T}$ & $\mathbf{T} / \mathbf{T}$ & \\
\hline Age (years) & & $47(25-63)$ & $45(23-62)$ & $41(29-63)$ & 0.65 \\
\hline Males $(\%)$ & & 74 & 63 & 43 & 0.15 \\
\hline DM (\%) & & 10 & 8 & 14 & 0.88 \\
\hline dp-ucMGP (pmol/L) & & $1315(755-2737)$ & $1191(650-2478)$ & $1329(799-1998)$ & 0.57 \\
\hline$M G P$ mRNA $(\mathrm{RQ})^{\ddagger}$ & & $1.2(0.4-3.1)$ & $0.9(0.4-1.8)$ & $0.6(0.5-0.8)$ & 0.16 \\
\hline CAC score $(\mathbf{A U})^{\#}$ & & $15(0-1312)$ & $0(0-990)$ & $0(0-12)$ & 0.06 \\
\hline \multirow[t]{4}{*}{ Media VC score, $(\%)^{a}$} & $\mathbf{0}$ & 15 & 15 & 29 & 0.05 \\
\hline & 1 & 35 & 62 & 57 & \\
\hline & 2 & 33 & 15 & 0 & \\
\hline & 3 & 17 & 9 & 14 & \\
\hline \multicolumn{6}{|c|}{ rs1800802 } \\
\hline & & $\mathbf{A} / \mathbf{A}$ & $\mathbf{A} / \mathbf{G}$ & $\mathbf{G} / \mathbf{G}$ & \\
\hline Age (years) & & $45(23-62)$ & $47(25-64)$ & $49(36-60)$ & 0.59 \\
\hline Males (\%) & & 70 & 64 & 70 & 0.85 \\
\hline DM (\%) & & 9 & 12 & 0 & 0.62 \\
\hline dp-ucMGP (pmol/L) & & $1323(766-2526)$ & $1232(654-2016)$ & $1121(848-1884)$ & 0.78 \\
\hline$M G P$ mRNA $(\mathrm{RQ})^{\neq}$ & & $1.0(0.5-2.1)$ & $1.3(0.3-2.0)$ & $1.3(0.9-1.6)$ & 0.88 \\
\hline CAC score $(\mathbf{A U})^{\#}$ & & $0(0-1061)$ & $12(0-1021)$ & $15(14-1710)$ & 0.27 \\
\hline \multirow[t]{4}{*}{ Media VC score, $(\%)^{a}$} & $\mathbf{0}$ & 17 & 16 & 0 & 0.35 \\
\hline & 1 & 54 & 39 & 40 & \\
\hline & 2 & 15 & 34 & 40 & \\
\hline & 3 & 14 & 10 & 20 & \\
\hline
\end{tabular}

Data presented as median (range of 10th-90th percentile) or percentage. Differences between genotype groups were evaluated with Kruskal-Wallis and Pearson's Chi-square tests. ${ }^{*} \mathrm{n}=41 ;{ }^{*} \mathrm{n}=91 ;{ }^{n} \mathrm{n}=108$ 


\section{Chapter 7}

General discussion 


\section{General discussion}

The vessel wall is continuously exposed to stress, resulting in vascular remodeling. Arterial remodeling involves numerous molecular pathways regulating vascular repair. During long-term stress the vessel wall cannot cope with this resulting in structural and biological changes that modify vessel wall characteristics. This pathological adaptation eventually results in vascular stiffness. One major contributor of arterial remodeling is the development of vascular calcification. Vascular calcification has long been neglected and was considered clinically irrelevant. However, it is nowadays appreciated that vascular calcification is a highly active and regulated process, with a major role of vascular smooth muscle cells (VSMCs).

In this thesis I studied the role of arterial remodeling processes and the downstream development of vascular stiffness and calcification. Additionally, I studied the interaction between arterial medial remodeling and the initiation and progression of atherosclerosis. In particular, I focused on the role of VSMCs in the process of vascular remodeling and calcification.

In chapter 2, I provide an extensive review on the role of VSMCs in arterial remodeling with a focus on calcification-related processes. In chapters 3 and 4 we report on in vivo mouse models and employed these to study the effect of medial remodeling on the development of atherosclerosis. In chapter 3 we investigated the effect of medial calcification on the development of atherosclerosis. In chapter 4 we studied the role of medial VSMC apoptosis on the development of atherosclerosis. In Chapter 5 I describe a novel BioHybrid in vitro approach to study the cardiovascular risk profile of patient plasma by using human primary VSMCs as biosensor for calcification. In chapter 6 we studied the association between vitamin K-dependent matrix Gla protein (MGP) and vascular calcification, and additionally the role of MGP as a biomarker.

The key findings of my thesis are:

(I) VKA induced medial calcification accelerates atherosclerosis development (chapter 3)

(II) VKAs induce extensive arterial remodeling in the medial layer of the vessel

(III) Apoptosis of medial VSMCs aggravate atherosclerosis and induces features of plaque instability (chapter 4)

(IV) The BioHybrid is a novel VSMC-based system using serum of patients to study their cardiovascular risk profile (chapter 5)

(V) Vitamin K-dependent MGP is an independent predictor of vascular calcification in patients with end stage renal disease (chapter 6)

\section{VKA induced development of medial calcification}

In my thesis I focus on mechanisms that lead to arterial remodeling. Vascular calcification is considered a late-stage result of arterial remodeling. It has been reported that VKA use is associated with increased vascular calcification ${ }^{1-3}$. Isolated medial calcification is predominantly found in $\mathrm{CKD}^{4}$, Mönckeberg Sclerosis ${ }^{5}$, breast calcification ${ }^{6}$ and calciphylaxis patients ${ }^{7}$ but often coincides with atherosclerosis. Additionally, patients susceptible for the development of medial calcification, such as chronic kidney disease and diabetes patients, die regularly from cardiovascular disease (CVD) ${ }^{8,9}$. Indeed, some 80 years ago it has been reported that medial calcification or degeneration precedes atherosclerosis ${ }^{10}$. 
Germline genetically engineered animals such as $\mathrm{LDLr}^{-/}$and Apoe ${ }^{-/-}$mice, are preferred to study atherosclerosis ${ }^{11,12}$. DBA/2 mice, that are susceptible for the development of ectopic calcification, do not develop atherosclerosis ${ }^{13}$. We designed a novel experimental animal model that develops VKA induced medial vascular calcification ${ }^{14}$ with subsequent induction of atherosclerosis by introducing LDL receptor knock-down. We employed the gain-of-function proprotein convertase subtilisin/kexin type 9 (PCSK9), that binds hepatic low-density lipoprotein (LDL) receptors directing them to the lysosomes for degradation ${ }^{15}$. Employment of PCSK9 gain of function in DBA/2 mice with medial calcification raised lipid levels to that of $\mathrm{LDLr}^{-/}$and Apoe ${ }^{-/-}$mice. Additionally, plaque development was found in the aorta and side-branches. We found that VKA use induces arterial medial remodeling and calcification which results in increased atherosclerosis and intimal calcification. Currently, the model is employed to unravel the mechanisms by which VSMCs are involved in medial remodeling and have an impact on atherosclerosis.

\section{VSMC apoptosis aggravates atherosclerosis progression and induces features of plaque instability}

Maintaining vessel homeostasis involves a balance between proliferation and apoptosis of VSMCs. After vessel injury VSMC apoptosis occurs, followed by increased proliferation. The fact that after vessel injury normal medial cellularity is achieved, indicates that VSMC apoptosis triggers repopulation of the vessel ${ }^{16}$. Additionally, vascular diseases such as aneurysm ${ }^{17}$ and atherosclerosis ${ }^{18}$ are associated with increased VSMC apoptosis, resulting in inflammation, endothelial damage and blood flow oscillations. My work confirms that loss of medial VSMCs results in larger atherosclerotic plaques with features of a more vulnerable phenotype. Our observations extend previous findings demonstrating that the induction of VSMC apoptosis in established atherosclerosis increased both plaque size and features of plaque vulnerability ${ }^{19,20}$. An explanation for the increase in plaque size is the observed thinner fibrous cap, which facilitates increased leukocyte infiltration, plaque inflammation and necrotic core formation. The fibrous cap consists of VSMCs that stabilize the atherosclerotic plaque. Because of medial VSMC apoptosis, it is plausible that migration of medial VSMCs towards the fibrous cap is impaired. Our data are in line with data from Clarke and coworkers who showed that intimal VSMC apoptosis resulted in thinning of the fibrous cap with subsequent necrotic core enlargement. A thin fibrous cap is characteristic of advanced human atherosclerosis, and plaques with thin fibrous caps are associated with increased risk on rupture ${ }^{21}$. Additionally, VSMCs have efficient efferocytosis capacity that can contribute to clearance of debris in atherosclerotic plaques 22 . The decline in efferocytosis capacity combined with the lower number of VSMCs might further accelerate atherosclerotic plaque growth.

We also found that extensive medial VSMC apoptosis did not affect vessel contractility. This finding suggests that the remaining VSMCs become hypertrophic and compensate for VSMC loss and maintain vessel wall contractility. Indeed, VSMC contractility markers were increased in animals with increased VSMC apoptosis. Vessel wall distensibility however, was compromised and vessel dilation was reduced compared to healthy vessels. These effects likely caused by a loss of endothelial derived hyperpolarizing factors. The exact mechanism by which this decrease in vessel wall 
dilation is caused is not fully understood but might arise from the impaired cellular connections in gap junctions between endothelial cells and VSMCs ${ }^{23}$.

\section{Development of the BioHybrid system - using in vitro VSMCs to measure cardiovascular risk}

To predict risk for cardiovascular disease, circulating biomarkers for vascular calcification are being used. However, this circulating compartment comprises not a single component but the collection of all circulating components. Therefore, we developed an assay that enables us to measure the cardiovascular risk profile of whole patient serum using VSMCs in vitro as detection system. The BioHybrid system is able to measure in vitro VSMC calcification using serum of patients prone to develop vascular calcification. Current in vitro VSMC models for determining calcification are end-point assays that measure calcification at a subjectively chosen time-point. Our BioHybrid assay provides a reproducible, sensitive and human VSMC-based calcification assay that can be monitored in time, and measures the effect of circulating components on the vascular system. Current clinical determination of vascular calcification includes $\mathrm{CT}$ and PET imaging while remodeling, a feature of vascular calcification, can be measured via PWV and IMT. All techniques are limited to late stages of vascular calcification development. Thus, we aim for applying the Biohybrid screening system as a method to detect early stages of vascular calcification. Several patient populations, including CKD ${ }^{24}, \mathrm{PXE}^{25}$ and diabetes patients ${ }^{8}$ are known to be prone to develop vascular calcification. In our Biohybrid system we tested serum from CKD and PXE patients and demonstrated its applicability as an indicative readout based on the cardiovascular status of these patients. Indeed, most patient serum/ plasma samples show a large responsiveness in our platform. Patients that display vascular calcification are largely heterogenous in nature which is reflected in our Biohybrid platform. Not all patients measured respond in a similar manner, opposite responsiveness in the Biohybrid system is a powerful tool to screen for factors that determine the calcifying potential and enables us to stratify patients in better way. Furthermore, the sensitivity of the assay has been demonstrated in response to dialysis, vitamin $\mathrm{K}$ treatment and both metabolic and genetic disorders.

\section{The role of vitamin K-metabolism on arterial remodeling in the clinical situation}

Clinical assessment of arterial remodeling processes is challenging. Assessment tools include measurements that determine vessel characteristics such as pulse wave velocity, distensibility and intima-media thickness, and imaging modalities like computed tomography (CT) and positron emission tomography (PET). Additionally, biomarkers that can predict arterial remodeling or vascular calcification are scarce and not specific. Recently, cellular senescence (measured as $\mathrm{CDK} 2 \mathrm{~A} / \mathrm{p} 16^{\mathrm{INK} 4 \mathrm{a}}$ by immunohistochemistry and mRNA expression) has been suggested as a marker for biological ageing ${ }^{26}$. CKD patients are characterised by accelerated ageing expressed as progressive vascular disease with increased vascular calcification. In CKD the arterial wall appears older than its expected age due to mechanisms that lead to premature ageing, such as inflammation, oxidative stress and cellular senescence. Indeed, high levels of senescence were found in CKD patients and were associated with vascular calcification ${ }^{27}$. Several biomarkers for vascular calcification have been reported so far and sclerostin ${ }^{28,29}$, Fetuin- $\mathrm{A}^{30}$ and MGP ${ }^{31}$ are amongst the most studied. Additionally, in the clinic distinction between medial and intimal calcification is not yet possible. In 
this thesis we focused on MGP as it is the only factor that can be modified activity wise. We found that the inactive form, dephosphorylated uncarboxylated MGP (dp-ucMGP), was an independent predictor of both intimal and medial vascular calcification in CKD patients. In accordance with our results, dp-ucMGP has been found to correlate with the amount of vascular calcification ${ }^{32-34}$. Therewith, we found increased local ucMGP tissue and mRNA MGP expression that associates with both the plasma dp-ucMGP as well as CAC levels. We are the first to show that MGP is an important player in the development of vascular calcification. Not only does the plasma dp-ucMGP level associate with medial and intimal calcification we also found increased gene and local tissue expression. Most literature reports only dp-ucMGP levels to be associated with vascular calcification. We believe MGP to be a potent inhibitor of vascular calcification and a promising target to modulate vascular calcification.

One of the strongest and accepted risk-factors for CVD development are increased blood lipid levels. Statins have proven to be effective in lowering LDL cholesterol and have shown to reduce CVD incidence and progression among different risk categories, in men and woman, and younger and older individuals ${ }^{35,36}$. However, statin use has also been associated with the development of vascular calcification ${ }^{37-39}$. Statins inhibit the enzymatic activity of UBIAD1, an enzyme important for vitamin K synthesis ${ }^{40}$. Therefore, it is tempting to speculate that patients treated with statins are prone to develop vitamin K deficiency. Indeed, CKD patients have been shown to be severely vitamin $\mathrm{K}$ deficient ${ }^{41}$. MGP requires vitamin $\mathrm{K}$ as cofactor to be posttranslationally activated, and increased dp-ucMGP levels reflect vitamin Kdeficiency ${ }^{42}$. We hypothesize that statin use accelerates vascular calcification via inducing vitamin K-deficiency, thereby increasing dp-ucMGP levels. Indeed, literature has shown that in CKD5D patients, statin use was associated with CAC progression and that statins blocked vitamin $\mathrm{K}$ synthesis in vitro. Moreover, dp-ucMGP plasma levels were associated with increased CAC score and medial vascular calcification ${ }^{43}$.

\section{Concluding remarks and future perspectives}

In my thesis I discussed arterial remodeling from a basic, translational and clinical research point of view. Today, CVD treatment is based on resolving symptoms rather than preventing disease development. This is likely caused by late clinical diagnose which can only detect severe stages of malformations. In this thesis, I discussed the importance of arterial remodeling in relation to development of CVD. In chapters 3 and 4 we report on novel animal models representing arterial medial remodeling, i.e. vascular calcification and apoptosis. These models aid the understanding how vascular remodeling affects atherogenesis thereby resulting in possibly CVD-related processes at an earlier stage, even before CVD is detected. Our work puts forward a key role for VSMC phenotypic switching, which plays a pivotal role in vessel repair. Our work revealed that VSMC apoptosis resulted in phenotype change of remaining VSMCs, increasing expression of contractile markers and becoming hypertrophic. This compensatory mechanism resulted in increased vessel contractility. Additionally, my data indicate a strong correlation between vascular calcification and development and progression of atherosclerosis. Vascular medial remodeling especially affects extracellular matrix (ECM) content with debranching, thinning and breaks of elastic fibers. In chapter 5, I describe the generation of a novel method to assess the cardiovascular risk profile using the in vitro BioHybrid assay. The BioHybrid assay is 
used to measure in vitro calcification that reflects in vivo vascular calcification. Our data point towards using the whole circulating compartment, rather than a singular biomarker, to assess the cardiovascular risk profile. Moreover, the Biohybrid platform can be extended incorporating multiple assays and cell-reporters that can provide a risk profile for CVD and beyond. Furthermore, the Biohybrid assay may have the potential to be a prediction tool for detecting vulnerable patients. Although we provide data pointing towards using the whole serum compartment for in vitro research, single biomarker assessment is still essential and can be used to reflect deficiencies. As such, in chapter 6 the use of dp-ucMGP as biomarker for vitamin K-status to predict vascular calcification was described. Using MGP as an imaging tool is appealing as it potentially demonstrates processes leading to calcification well before the calcification can be detected. As such, MGP may provide an unique imaging tool that increases resolution and interpretation of vascular calcification. Taken together, the data in my thesis show that arterial remodeling is key in the development of vascular calcification and atherosclerosis and that early detection and intervention is necessary to treat CVD.

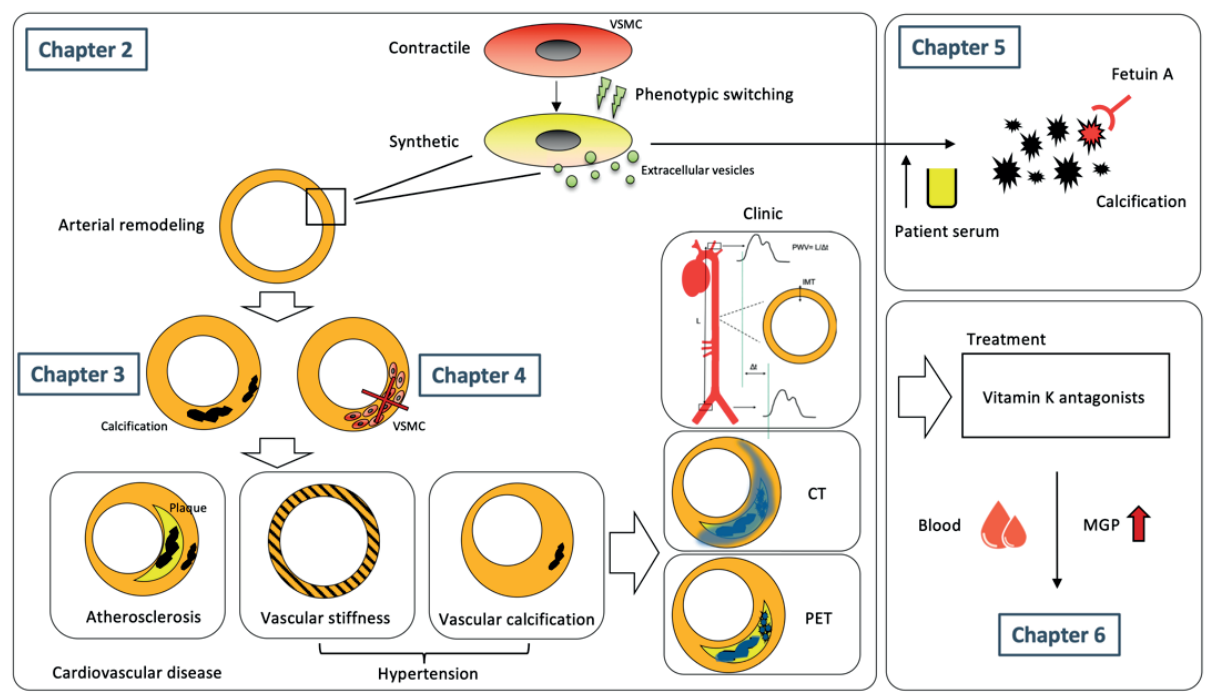

Concluding figure. Overview of the chapters in this thesis. Chapter 1 is the general introduction. General mechanisms of arterial remodeling are described in Chapter 2. Briefly, arterial remodeling is initiated by VSMCs, the cell type that is most abundant in the vessel wall. VSMCs display a contractile phenotype to maintain vessel homeostasis. Upon stress signals, VSMCs change towards a synthetic phenotype to repair damage inflicted. Long-term damage leads to pathology development such as atherosclerosis, arterial stiffness and vascular calcification. Clinically, arterial remodeling cannot be measured accurately. Measuring vascular calcification currently includes pulse wave velocity (indirect), computation tomography and positron emission tomography using ${ }^{18} \mathrm{~F}-\mathrm{NaF}$. Patients that display vascular calcification are often treated with Vitamin $\mathrm{K}$ antagonists (VKAs) that are involved in anticoagulation. We found that VKA use (Chapter 6) increases vascular calcification and the inactive form of matrix Gla protein. To study arterial remodeling processes more detailed we developed two animal models that focus on VSMC phenotypic switching. In Chapter 3 we induce VSMC-specific apoptosis and study its effect on atherogenesis. Chapter 4 describes a model in which we induce vascular calcification in the medial layer using VKAs. Last, we aimed to developed an assay able to measure a patients' propensity to calcify (Chapter 5). We validated this new method in highly susceptible patient populations. 


\section{References}

1. Koos, R. et al. Relation of oral anticoagulation to cardiac valvular and coronary calcium assessed by multislice spiral computed tomography. Am. J. Cardiol. 96, 747-749 (2005).

2. Chatrou, M. L. L., Winckers, K., Hackeng, T. M., Reutelingsperger, C. P. \& Schurgers, L. J. Vascular calcification: The price to pay for anticoagulation therapy with vitamin $\mathrm{K}$-antagonists. YBLRE 26, 155-166 (2012).

3. Peeters, F. E. C. M. et al. Vitamin K Antagonists, Non-Vitamin K Antagonist Oral Anticoagulants, and Vascular Calcification in Patients with Atrial Fibrillation. TH Open 2, e391-e398 (2018).

4. Moe, S. M. et al. Medial artery calcification in ESRD patients is associated with deposition of bone matrix proteins. Kidney Int. 61, 638-647 (2002).

5. Micheletti, R. G., Fishbein, G. A., Currier, J. S. \& Fishbein, M. C. Mönckeberg sclerosis revisited: a clarification of the histologic definition of Mönckeberg sclerosis. Arch. Pathol. Lab. Med. 132, 4347 (2008).

6. Trimboli, R. M., Codari, M., Guazzi, M. \& Sardanelli, F. Screening mammography beyond breast cancer: breast arterial calcifications as a sex-specific biomarker of cardiovascular risk. Eur J Radiol 119, 108636 (2019).

7. Rogers, N. M., Teubner, D. J. O. \& Coates, P. T. H. Calcific uremic arteriolopathy: advances in pathogenesis and treatment. Semin Dial 20, 150-157 (2007).

8. Edmonds, M. E. Medial arterial calcification and diabetes mellitus. Z Kardiol 89 Suppl 2, 101-104 (2000).

9. Garimella, P. S. \& Sarnak, M. J. Cardiovascular disease in CKD in 2012: moving forward, slowly but surely. Nat Rev Nephrol 9, 69-70 (2013).

10. Blumenthal, H. T., Lansing, A. I. \& Wheeler, P. A. Calcification of the Media of the Human Aorta and Its Relation to Intimal Arteriosclerosis, Ageing and Disease. The American Journal of Pathology 20, 665-687 (1944)

11. Getz, G. S. \& Reardon, C. A. Animal models of atherosclerosis. Arteriosclerosis, Thrombosis, and Vascular Biology 32, 1104-1115 (2012).

12. Stylianou, I. M., Bauer, R. C., Reilly, M. P. \& Rader, D. J. Genetic basis of atherosclerosis: insights from mice and humans. Circ. Res. 110, 337-355 (2012).

13. Paigen, B., Ishida, B. Y., Verstuyft, J., Winters, R. B. \& Albee, D. Atherosclerosis susceptibility differences among progenitors of recombinant inbred strains of mice. Arteriosclerosis 10, 316-323 (1990).

14. Kruger, T. et al. Warfarin Induces Cardiovascular Damage in Mice. Arteriosclerosis, Thrombosis, and Vascular Biology 33, 2618-2624 (2013).

15. Bjørklund, M. M. et al. Induction of atherosclerosis in mice and hamsters without germline genetic engineering. Circ. Res. 114, 1684-1689 (2014).

16. Malik, N. et al. Apoptosis and cell proliferation after porcine coronary angioplasty. Circulation $\mathbf{9 8 ,}$ 1657-1665 (1998).

17. López-Candales, A. et al. Decreased vascular smooth muscle cell density in medial degeneration of human abdominal aortic aneurysms. The American Journal of Pathology 150, 993-1007 (1997).

18. Lutgens, E. et al. Biphasic pattern of cell turnover characterizes the progression from fatty streaks to ruptured human atherosclerotic plaques. Cardiovasc. Res. 41, 473-479 (1999).

19. Clarke, M. C. H. et al. Apoptosis of vascular smooth muscle cells induces features of plaque vulnerability in atherosclerosis. Nat. Med. 12, 1075-1080 (2006).

20. Clarke, M. C. H. et al. Chronic apoptosis of vascular smooth muscle cells accelerates atherosclerosis and promotes calcification and medial degeneration. Circ. Res. 102, 1529-1538 (2008).

21. Burke, A. P. et al. Coronary risk factors and plaque morphology in men with coronary disease who died suddenly. N. Engl. J. Med. 336, 1276-1282 (1997).

22. Allahverdian, S., Pannu, P. S. \& Francis, G. A. Contribution of monocyte-derived macrophages and smooth muscle cells to arterial foam cell formation. Cardiovasc. Res. 95, 165-172 (2012).

23. Sandow, S. L., Senadheera, S., Bertrand, P. P., Murphy, T. V. \& Tare, M. Myoendothelial contacts, gap junctions, and microdomains: anatomical links to function? Microcirculation 19, 403-415 (2012).

24. Wang, X.-R., Zhang, J.-J., Xu, X.-X. \& Wu, Y.-G. Prevalence of coronary artery calcification and its association with mortality, cardiovascular events in patients with chronic kidney disease: a systematic review and meta-analysis. 41, 244-256 (2019).

25. Gheduzzi, D. et al. Matrix Gla protein is involved in elastic fiber calcification in the dermis of pseudoxanthoma elasticum patients. Lab. Invest. 87, 998-1008 (2007). 


\section{Chapter 7}

26.

28. Figurek, A., Rroji, M. \& Spasovski, G. Sclerostin: a new biomarker of CKD-MBD. Int Urol Nephrol 382, 260-7 (2019).

29. Evenepoel, P. et al. Sclerostin Serum Levels and Vascular Calcification Progression in Prevalent Renal Transplant Recipients. J. Clin. Endocrinol. Metab. 100, 4669-4676 (2015).

30. Ketteler, M. et al. Association of low fetuin-A (AHSG) concentrations in serum with cardiovascular mortality in patients on dialysis: a cross-sectional study. The Lancet 361, 827-833 (2003).

31. Schurgers, L. J., Uitto, J. \& Reutelingsperger, C. P. Vitamin K-dependent carboxylation of matrix Gla-protein: a crucial switch to control ectopic mineralization. Trends Mol Med 19, 217-226 (2013).

32. Rennenberg, R. J. M. W. et al. Vascular calcifications as a marker of increased cardiovascular risk: a meta-analysis. Vasc Health Risk Manag 5, 185-197 (2009).

33. Schurgers, L. J. et al. The circulating inactive form of matrix gla protein is a surrogate marker for vascular calcification in chronic kidney disease: a preliminary report. Clin J Am Soc Nephrol 5, 568575 (2010)

34. Roumeliotis, S., Dounousi, E., Eleftheriadis, T. \& Liakopoulos, V. Association of the Inactive Circulating Matrix Gla Protein with Vitamin K Intake, Calcification, Mortality, and Cardiovascular Disease: A Review. Int J Mol Sci 20, 628 (2019).

35. Katzmann, J. L. \& Laufs, U. New Insights in the Control of Low-Density Lipoprotein Cholesterol to Prevent Cardiovascular Disease. Curr Cardiol Rep 21, 69 (2019).

36. Cholesterol Treatment Trialists' Collaboration. Efficacy and safety of statin therapy in older people: a meta-analysis of individual participant data from 28 randomised controlled trials. Lancet 393, 407415 (2019)

37. Nakazato, R. et al. Statins use and coronary artery plaque composition: results from the International Multicenter CONFIRM Registry. Atherosclerosis 225, 148-153 (2012).

38. Henein, M. et al. High dose and long-term statin therapy accelerate coronary artery calcification. Int. J. Cardiol. 184, 581-586 (2015).

39. Andrews, J., Psaltis, P. J., Bartolo, B. A. D., Nicholls, S. J. \& Puri, R. Coronary arterial calcification: A review of mechanisms, promoters and imaging. Trends Cardiovasc. Med. 28, 491-501 (2018).

40. Hirota, Y. et al. Functional characterization of the vitamin K2 biosynthetic enzyme UBIAD1. PLoS ONE 10, e0125737 (2015).

41. Cranenburg, E. C. M. et al. Vitamin K intake and status are low in hemodialysis patients. Kidney Int. 82, 605-610 (2012).

42. Cranenburg, E. C. M. et al. Characterisation and potential diagnostic value of circulating matrix Gla protein (MGP) species. Thromb. Haemost. 104, 811-822 (2010).

43. Chen, Z. et al. Does statins promote vascular calcification in chronic kidney disease? Eur. J. Clin. Invest. 47, 137-148 (2017). 


\section{Chapter 8}

Summary 
In this thesis the main focus was to investigate the role of vascular smooth muscle cells (VSMCs) in vascular remodeling with a special focus on vascular calcification. In chapter 1 we delineate the impact of cardiovascular disease (CVD) on society. One of the key players that contributes to CVD development is vascular remodeling. Vascular remodeling reflects the adaptation of the vessel wall to biochemical and biomechanical stimuli. In general, two types of remodeling are discriminated: outward and inward remodeling. Vascular remodeling ultimately results in CVD diseases such as atherosclerosis and vascular calcification and arterial stiffening. The VSMC, being the most abundant cell type in the vessel wall, contributes for a major part to the development and progression of vascular remodeling. Processes such as proliferation, migration, extracellular vesicle (EV) release and apoptosis are biological pathways that are regulated by VSMCs. Chapter 2 focusses in more detail on vascular remodeling processes and especially the role that VSMCs have in its regulation. VSMC phenotypic switching is the most important factor that contributes to vascular remodeling. Under physiological condition VSMCs display a contractile phenotype characterised by high expression of markers involved in contraction and dilation. Upon stress VSMCs respond by changing their phenotype in order to conduct vessel repair. Environmental cues, such as soluble biochemical compounds, extracellular matrix (ECM) proteins and biophysical conditions interact with VSMCs and their phenotypic state. Additionally, special emphasize is given on clinical features of vascular remodeling. Extensive vascular remodeling can lead to pathologies such as hypertension, vascular calcification, atherosclerosis and fibrosis. These clinical manifestations of vascular remodeling are extensively discussed in chapter 2 with a special emphasize on the role of the VSMCs in these diseases. Last, current clinical detection methodologies of vascular remodeling including their strengths and limitations are discussed.

There is a lack of good models to study vascular remodeling, and most models that currently exist focus on diseases themselves, rather than on arterial remodeling processes. In chapter 3 we developed a model in which we specifically induced medial calcification by the use of Vitamin K antagonists (VKAs). Medial calcification is a late stage remodeling feature and it is difficult to study without developing vascular calcification at other sites of the vascular tree. We found that VKAs induce cardiovascular calcification, including ectopic heart calcification, valvar calcification and medial calcification of the aorta. Additionally, vessel compliance was decreased and isolated aortas showed increased uptake of $18 \mathrm{~F}-\mathrm{NaF}$ a radionuclide tracer that is reported to image active mineralization. Furthermore, we studied the effect of established medial calcification on subsequent atherosclerosis development, and found that intimal calcification was significantly increased. Moreover, increased uncarboxylated matrix Gla protein (MGP) and features of extensive vascular remodeling were found.

In chapter 4 we studied the effect of VSMC apoptosis on the development of atherosclerosis. Here we exploited a different model of vascular remodeling, we used SM22 $\square$-hDTr+/ApoE-/- mice to induce VSMC apoptosis. Upon DT exposure, VSMC cell numbers were decreased up to 50\% compared to control littermates. In SM22 $\square$ $\mathrm{hDTr}+/$ ApoE-/- mice we found that the remaining VSMCs showed signs of increased contractility and hypertrophy, both in the thoracic aorta and in ex vivo cultured VSMCs. In $\mathrm{hDTr}+$ mice we found that vessel contractility was preserved while vessel dilation capability was impaired. Upon atherosclerosis induction (6 and 18 weeks) SM22 $\square-$ $\mathrm{hDTr}+/ \mathrm{ApoE}-/$ - mice developed significantly larger atherosclerotic plaques, with 
increased inflammation ( 6 weeks), a thinner fibrous cap ( 6 and 18 weeks), lower intimal VSMCs ( 6 weeks) and a larger necrotic core (18 weeks). These are all hallmarks of a more vulnerable plaque phenotype.

To gain further insight in the mechanisms of vascular calcification development, we used isolated human primary VSMCs and developed a novel in vitro calcification assay that measures kinetic calcification development. In chapter $\mathbf{5}$ we compare this novel assay to existing methods for calcification determination. One major advantage over other assays is the ability to measure calcification development over time. This BioHybrid platform can be used for both human serum and plasma samples. The Biohybrid platform was assessed by the use of samples of patients known to be susceptible for the development of vascular calcification, such as chronic kidney disease (stages III, IV, V and VD; CKD), pseudoxanthoma elasticum, high coronary artery calcium (CAC) score patients, VKA users and aortic valve calcification patients. For most of these patients the BioHybrid platform was able to detect a high calcification propensity compared to controls.

As biomarker is still valuable for clinical research we studied, in chapter 6, the role of MPG as a biomarker for vascular calcification in CKD patients. The most inactive form of MGP (dephosphorylated uncarboxylated MGP; dp-ucMGP) was shown to associate with increased CAC and medial calcification score. Additionally, at areas of extensive medial calcification, ucMGP positivity was found. In multivariate analyses dpucMGP was associated to bone markers and medial calcification score was associated with age, gender, diabetes mellitus and dp-ucMGP levels. Moreover, MGP mRNA expression was shown to associate high CAC score and with high dp-ucMGP levels in the plasma. Last, we sought to determine the role of MGP single nucleotide polymorphisms in relation to the development of vascular calcification. We could not find clear MGP SNP that associated with vascular calcification. 


\section{Chapter 9}

Nederlandse samenvatting 
Het hoofddoel in mijn proefschrift was het onderzoeken van de rol van vasculaire gladde spiercellen (VSMCs) in vasculaire remodellering met een speciale focus op vasculaire verkalking. In hoofdstuk 1 besprak ik de impact van hart- en vaatziekten (CVD) op de samenleving. Een van de belangrijkste spelers die bijdragen aan CVD is vasculaire remodellering. Vasculaire remodellering is het aanpassingsvermogen van de vaatwand op biochemische en biomechanische prikkels. In het algemeen zijn er twee typen van remodellering te onderscheiden: buiten- en binnenwaartse remodellering. Omvangrijke vasculaire remodellering leidt uiteindelijk tot CVD, zoals atherosclerose en arteriële stijfheid, die beiden worden gekenmerkt door vasculaire verkalking. VSMCs zijn de meest voorkomende cellen in de vaatwand en dragen voor een groot deel bij aan de ontwikkeling en progressie van vasculaire remodellering. Biologische processen zoals proliferatie, migratie, extracellulair vesicle (EV) afgifte en apoptose worden door VSMCs gereguleerd. Hoofdstuk 2 gaat meer gedetailleerd in op de rol van VSMCs in vasculaire remodellering. VSMC fenotypische switch is een van de belangrijkste factoren die bijdraagt aan vasculaire remodellering. Onder fysiologische condities hebben VSMCs een contractiel fenotype dat wordt gekarakteriseerd door een hoge expressie van markers die belangrijk zijn bij de contractie en dilatatie van de vaatwand. Bij overmatige stress reageren de VSMCs en veranderen deze hun fenotype om de vaatwand te herstellen. Omgevingsfactoren, zoals oplosbare biochemische stoffen, extracellulaire matrix eiwitten en biofysische condities hebben interactie met de VSMC en hun fenotype. Bovendien, wordt er in hoofdstuk 2 speciale nadruk gelegd op klinische aspecten van vasculaire remodellering. Een grote mate van vasculaire remodellering kan leiden tot ziektebeelden zoals hypertensie, atherosclerose, arteriële stijfheid, vasculaire verkalking en fibrose. Als laatste, wordt de huidige diagnostiek van vasculaire remodellering besproken met daarbij hun sterkte- en zwaktepunten.

Preklinisch is er een gebrek aan goede modellen voor het bestuderen van vasculaire remodellering. In hoofdstuk 3 hebben wij een diermodel ontwikkeld waarin we mediale verkalking kunnen opwekken door het gebruik van vitamine $\mathrm{K}$ antagonisten (VKA). Mediale verkalking is een kenmerk van vasculaire remodellering dat laat tot uitdrukking komt en is moeilijk te bestuderen in onderzoek dat zich focust op atherosclerose. In dit onderzoek lieten wij zien dat VKA cardiovasculaire verkalking opwekken, inclusief ectopische hart verkalking, klep verkalking en mediale verkalking van de aorta. Bovendien, was vaatwand compliantie verlaagd en zagen we een verhoogde opname van $18 \mathrm{~F}-\mathrm{NaF}$, een radionuclide PET tracer die gebruikt wordt bij het in beeld brengen van actieve verkalking. Verder, hebben wij gebruik gemaakt van het "gain of function" AAV virus dat het PCSK9 eiwit verhoogd en hebben wij het effect van mediale verkalking op de ontwikkeling van atherosclerose bestudeerd. In dit model hebben we laten zien dat het aanwezig zijn mediale verkalking leidt tot een significante toename van zowel atherosclerose als intimale verkalkingen. Bovendien, was er een toename van niet-gecarboxyleerd matrix Gla eiwit (MGP) en vonden wij kenmerken van extensieve vasculaire remodellering.

In hoofdstuk $\mathbf{4}$ hebben wij het effect van VSMC apoptose op atherosclerose ontwikkeling bestudeerd. We hebben hier een ander diermodel gebruikt, namelijk SM22 $\square$-hDTr+/ApoE-/- muizen waarin we specifiek VSMC in apoptose kunnen brengen. Na het toedienen van DT, verminderde het aantal VSMCs in de vaatwand met $50 \%$ t.o.v. controle nestgenoten. In SM22 $\square-\mathrm{hDTr}+/ \mathrm{ApoE}-/-$ muizen hebben wij laten 
zien dat de achtergebleven VSMCs een verhoogde contractiliteit hebben en hypertroof zijn, zowel in de aorta als in ex vivo geïsoleerde cellen. Verder lieten we zien dat SM22 $\square$-hDTr+/ApoE-/- muizen eenzelfde vaatwand contractiliteit hadden maar dat vaatwand dilatatie was verminderd. Na het opwekken van atherosclerose (zowel vroege als late atherosclerotische plaques) ontwikkelden SM22 $\square-\mathrm{hDTr}+/ \mathrm{ApoE}-/-$ muizen significant grotere atherosclerotische plaques, met verhoogde ontsteking (vroeg), een dunnere fibrotische cap (beide vroeg en laat), minder intimale VSMCs (vroeg) en een grotere necrotische kern (laat). Al deze eigenschappen zijn kenmerken van een meer kwetsbaar plaque fenotype.

Om verder inzicht te verschaffen in de mechanismen van de ontwikkeling van vasculaire verkalking, hebben wij een nieuwe kinetische in vitro calcificatie assay ontwikkeld die gebruik maakt van geïsoleerde humane VSMCs. In hoofdstuk $\mathbf{5}$ hebben wij deze assay vergeleken met bestaande methoden voor het meten van verkalking. Een groot voordeel van onze assay is dat we verkalking kunnen meten over tijd. Ons "BioHybrid" platform kan worden gebruikt voor zowel humaan serum als plasma. Het BioHybrid platform is gevalideerd door serum en plasma samples van patiënten te gebruiken die klinisch hebben bewezen gevoelig te zijn voor het ontwikkeling van verkalkingen, zoals: chronische nierziekten (stadium III, IV, V en VD; CKD), pseuxanthoma elasticum, hoge coronair arterie calcium (CAC) score patiënten, VKA gebruikers en patiënten met klepverkalking. Over het algemeen was het BioHybrid platform bekwaam om patiënten met een hoge verkalking neiging op te kunnen pikken vergeleken met controles.

Biomarkers blijven een belangrijke rol spelen in klinisch onderzoek en dus hebben we in hoofdstuk 6 de rol van MGP als biomarker voor verkalking in CKD patiënten onderzocht. De inactive vorm van MGP (gedefosforyleerd-nietgecarboxyleerd MGP; dp-ucMGP) werd geassocieerd met een hoge CAC en mediale verkalking score. Verder konden wij geen rol voor MGP single nucleotide polymorfismen vinden met vasculaire verkalking. Echter, bij gebieden die veel mediale verkalkingen vertoonden, was er een grote mate van ucMGP positiviteit. In multivariate analyses vonden wij dat plasma dp-ucMGP was geassocieerd met bot markers en dat mediale verkalking score was geassocieerd met leeftijd, geslacht, diabetes mellitus en plasma dp-ucMGP waarden. Tenslotte werden MGP mRNA levels geassocieerd met een hoge CAC score en met hoge dp-ucMGP waarden in het plasma. 


\section{Chapter 10}

Valorization 
Valorization, the utilization of academic research. Or the process of value creation out of knowledge and make it available for economic or societal purposes. Eventually so that it can be translated into products, service or other processes regarding industry.

In my thesis we studied the vascular smooth muscle cell (VSMC), a pivotal player in vascular health and the development of cardiovascular disease (CVD). In more detail we focused on its action in vascular remodeling processes that lead to the development of CVD and vascular calcification. CVD is still the number one cause of death in the world. And understanding the mechanisms of CVD is important, but does not help society directly. However, it can help in the development of new treatments strategies and earlier detection. Most clinical tools that are currently available diagnose CVD at a too late stage. Especially, vascular calcification is diagnosed overdue, when only treatment is focused around resolving the symptoms rather than curing the disease. The valorization part of my thesis focusses on the effect of medial remodeling processes that affect vascular calcification and CVD.

Chapter 2 elaborates on the role of VSMCs in arterial remodeling processes, with a focus on calcification-related processes. In this chapter the role of VSMCs in CVD is discussed from a fundamental, translational and clinical point of view. Chapter 3 investigates the effect of medial calcification on atherosclerosis development. We believe that medial degradation precedes the pathological build-up of plaques. This concept is already old, but recent literature investigates atherosclerosis mostly from a lipid point of view. The research in chapter 3 confirms that medial degeneration, induced as medial calcification by vitamin $\mathrm{K}$ antagonists (VKAs), leads to extensive extracellular medial remodeling with subsequent atherosclerosis induction. We believe it is important to disseminate this view with clinicians to change the way of treating CVD. Additionally, big pharmaceutical companies should change their focus on CVD drug discovery from relieving symptoms towards disease prevention. Another important finding in chapter 3 is that VKAs induce accelerated vascular calcification. VKAs are regularly prescribed to patients suffering from CVD, but clinicians are often not aware of the detrimental effects VKAs have on established atherosclerotic plaques. For example, numerous studies have shown that chronic kidney disease (CKD) patients display extreme vascular calcification after VKA use.

In chapter 4 we focused on the role of VSMCs apoptosis on the development of atherosclerosis. Our results indicate that VSMC apoptosis in the vessel media results in larger and more vulnerable atherosclerotic plaques. Furthermore, recent literature indicates that most cells that reside inside atherosclerotic plaques are derived from VSMC origin. These findings might have a great impact on the clinical situation. The most well-known treatments of atherosclerosis involve lipid lowering, by-pass surgery and stenting. It is pivotal to include the VSMC into current treatment, as an important contributor to the development and progression of atherosclerosis. Future treatment strategies should focus more on changing the phenotype from synthetic, excreting and proliferative VSMCs towards a contractile phenotype in which the proliferative and secretory nature is decreased.

Chapter 5 describes a novel method to measure calcification propensity using patient serum or plasma, and its susceptibility to develop vascular calcification. Current methods for calcification determination are unrefined and there is no consensus on how 
the calcification assays are performed. With our assay we are able to measure calcification kinetics and evaluate patient specific responses while restricting to optimal calcification conditions. The BioHybrid assay might potentially be used in future academic research as golden standard. Thereby, normalising and improving the current end-point assays that are available. Moreover, the BioHybrid platform can be expanded by more in vitro assays to cover a wide range of biological read-out parameters. This in combination with the use of several primary cell types provides a platform that is currently unique and non-existing, with the potential to not only combat vascular calcification but other diseases as well. Additionally, the BioHybrid platform is expected to be fully translatable into animal and healthy volunteer phase, making it a patentable solution that can be commercialized.

The concept of a biohybrid platform is appealing but should not attenuate research to new biomarkers. In chapter 6 we investigate the biomarker potential of matrix Gla protein (MGP). We found that MGP is an independent predictor of both intimal and medial vascular calcification is a specific cohort of CKD patients. Biomarker research is valuable for the clinic as they can directly be used to interpret parameters of patients. The most inactive variant of MGP (dephosphorylated-uncarboxylatedMGP; dpucMGP) reflects a patient' Vitamin K status. Vitamin $\mathrm{K}$ is key in the activation of Vitamin K dependent proteins (VKDP) that are known to be important in the coagulation cascade. However, other VKDP, such as MGP, are involved in the inhibition of mineralization. We found that dp-ucMGP plasma levels associate with local protein and mRNA expression and also medial calcification. Targeting MGP, and especially its activation, might prove a novel therapeutic target as it can inhibit or prevent local tissue mineralization at a much earlier stage of the disease. One strategy to activate MGP is to supplement Vitamin $\mathrm{K}$ to patients. Although critical care should be taken when supplementing Vitamin $\mathrm{K}$ to patients that require anticoagulation. Currently, several clinical trials are being conducted to study the beneficial effects of Vitamin K in CVD and specifically vascular calcification prevention.

Taken together, the data described in my thesis provide a new angle for the development of therapeutic strategies to target vascular calcification and atherosclerosis. Ideally, treatment shifts towards earlier stages of the disease in which regression or prevention is still possible. Treating patients earlier is advantageous but clinical detection should develop along. To aid detection, we build the BioHybrid platform which is based on a VSMC readout to patients' plasma or serum. Clinically treatment that prevents or regresses calcification would be greatly appreciated as it is a benefit for CVD mortality/ morbidity and expensive treatments costs. In conclusion this thesis, covers the whole spectra of research from fundamental to translational and clinical. Thereby some of the findings can be valuable for the interpretation of a different therapeutic approach or can be translated into products and services that can be commercialized. 


\section{Chapter 11}

Curriculum Vitae 


\section{About the author}

Armand Mario Gerard Jaminon was born on November 21st 1989 in Heerlen, The Netherlands. He graduated from Sophianum high school in Gulpen in 2008 completing the Science and Health profile.

After graduation he started to study Bio-Pharmaceutical Sciences at the University of Leiden, where he received his Bachelor's degree in August 2011. After completion of the Bachelor program he started a 2 year Master program in BioPharmaceutical Sciences with a specialization in Drug Delivery Technology and Biopharmaceutics. He obtained his Master's degree in August 2013 after completing an internship of 9 months at the department of Biopharmacy at the University of Leiden under the supervision of Prof. Dr. van Eck and an internship of 6 months at the department of Molecular Genetics at the University of Maastricht under the supervision of Dr. Neumann.

In 2013 he started to work as a researcher at the department of Molecular Genetics of the University of Maastricht under the supervision of Dr. Neumann. Here he performed research on the generation of stable knockout cell lines for AMPK subunits. In 2014 he started his $\mathrm{PhD}$ program at the department of Biochemistry at the University of Maastricht under the supervision of Prof. Dr. Schurgers and Prof. Dr. Reutelingsperger. During his PhD program he studied the role of vascular smooth muscle cells in vascular remodeling and calcification. During his $\mathrm{PhD}$ program he has published his data in peer-reviewed international scientific journals and presented his work at international conferences in Boston and Helsinki.

After his PhD he started to work as PostDoc on the RegMedXB project at the Biochemistry department of Maastricht University. A project that focusses on the regeneration of the human heart.

\section{Over de Auteur}

Armand Mario Gerard werd geboren op 21 november 1989 in Heerlen, Nederland. In 2008 behaalde bij zijn VWO diploma met het profiel Natuur en Gezondheid aan het Sophianum te Gulpen.

$\mathrm{Na}$ het afstuderen van de middelbare school begon hij aan zijn studie, biofarmaceutische wetenschappen aan de universiteit van Leiden, waar hij zijn bachelor behaalde in augustus 2011. Na het voltooien van zijn bachelor begon hij met een 2 jaar durende master opleiding bio-farmaceutische wetenschappen met een specialisatie in geneesmiddelen toediening technologie en biofarmacie. Hij behaalde zijn master in augustus 2013, na het voltooien van een stage van 9 maanden bij de afdeling biofarmacie aan de universiteit van Leiden onder supervisie van Prof. Dr. van Eck en een stage van 6 maanden bij de afdeling moleculaire genetica aan de universiteit van Maastricht onder supervisie van dr. Neumann.

In 2013 begon hij als onderzoeker bij de afdeling moleculaire genetica aan de universiteit van Maastricht onder supervisie van Dr. Neumann. Hier deed hij onderzoek naar de generatie van stabiele "knockout" cellijnen van AMPK sub-eenheden. In 2014 begon hij zijn promotie onderzoek bij de afdeling biochemie aan de univerisiteit van Maastricht onder leiding van Prof. Dr. Schurgers en Prof. Dr. Reutelingsperger. Tijdens zijn $\mathrm{PhD}$ bestudeerde hij de rol van gladde spiercellen in vasculaire remodellering en calcificatie. Tijdens zijn promotie traject publiceerde hij zijn data in peer-reviewed 
internationale wetenschappelijke tijdschriften en presenteerde hij zijn werk op internationale conferenties in Boston en Helsinki.

$\mathrm{Na}$ zijn promotie startte hij als PostDoc in het RegMed XB project op de afdeling biochemie van de universiteit van Maatricht. Het project focust zich op de regeneratie van het humane hart. 


\section{Chapter 12}

List of publications 


\section{Publications}

Jaminon AMG, Dai L, Qureshi AR, Evenepoel P, Ripsweden J, Söderberg M, et al. Matrix Gla protein is an independent predictor of both intimal and medial vascular calcification in chronic kidney disease. Sci Rep. Nature Publishing Group; 2020 Apr 20;10(1):6586-9.

Jaminon A, Reesink K, Kroon A, Schurgers L. The Role of Vascular Smooth Muscle Cells in Arterial Remodeling: Focus on Calcification-Related Processes. Int J Mol Sci. Multidisciplinary Digital Publishing Institute; 2019 Nov 14;20(22):5694.

Posthuma JJ, Posma JJN, van Oerle R, Leenders P, van Gorp RH, Jaminon AMG, et al. Targeting Coagulation Factor Xa Promotes Regression of Advanced Atherosclerosis in Apolipoprotein-E Deficient Mice. Sci Rep. Nature Publishing Group; 2019 Mar 7;9(1):3909.

Chen Z, Qureshi AR, Parini P, Hurt-Camejo E, Ripsweden J, Brismar TB, Barany P, Jaminon AMG, Schurgers LJ, Heimbürger O, Lindholm B, Stenvinkel P. Does statins promote vascular calcification in chronic kidney disease? Eur J Clin Invest. 2017 Feb;47(2):137-48.

Stöhr R, Schurgers L, van Gorp R, Jaminon A, Marx N, Reutelingsperger C. Annexin A5 reduces early plaque formation in ApoE -/- mice. PLoS ONE. Public Library of Science; 2017;12(12):e0190229.

van Kampen E, Jaminon A, van Berkel TJC, Van Eck M. Diet-induced (epigenetic) changes in bone marrow augment atherosclerosis. J Leukoc Biol. 2014 Nov;96(5):83341 .

\section{Publications (in preparation)}

Jaminon AMG, Rapp N, van Gorp R, Halder M, Leenders P, Bauwens M, Mottaghy F, Reesink K, Kramann R, Dijkgraaf I, Reutelingsperger CPM, Schurgers LJ. The role of medial vascular calcification on the development and progression of atherosclerosis. (in preparation)

Jaminon AMG, Willems BAG, van Zandvoort M, Recarti C, Schiffers PMH, Reesink K, Biessen EA, Clarke M, Bennett M, de Mey J, Vermeer C, Kramann R, Reutelingsperger CPM, Schurgers LJ. Medial vascular smooth muscle cell apoptosis accelerates atherogenesis and promotes features of a vulnerable plaque. (in preparation)

Jaminon AMG, Akbulut C, Kramann R, Biessen EA, Mees B, Natour E, Brandenburg $\mathrm{V}$, Jahnen-Dechent W, Floege J, Uitto J, Reutelingsperger CPM, Schurgers LJ. Use of primary vascular smooth muscle cells to predict vascular calcification potential of patients: Development of a BioHybrid Assay. (in preparation) 
van Gorp RH, Petsophonsakul P, Jaminon AMG, Leenders P, Jahnen-Dechent W, Reutelingsperger CPM, Schurgers LJ. Vitamin K inhibits vascular smooth muscle cell induced calcification via reduced oxidative stress and extracellular vesicle secretion. (in preparation)

Peeters FECM, Jaminon AMG, Peters D, Suverein M, Mesu J, Lorusso R, Segers P, Kats S, Crijns HGJM, Meex SJR, Kietselaer BLJH, Schurgers LJ. Role of calcification in progression of aortic valve stenosis: involvement of vitamin K-dependent Matrix Gla Protein. (in preparation)

Chatrou ML, Kusters DH, Willems BA, Jaminon AMG, Norling L, Proudfoot D, Spohn G, Spronk HM, Gruell H, Reutelingsperger CPM, Schurgers LJ. Vitamin K antagonists attenuate early and aggravate late atherogenesis in apoE-/- mice. (in preparation)

\section{Poster presentations}

Jaminon AMG, Willems BAG, van Gorp RH, Recarti C, Schiffers PMH, van Zandvoort M, Biessen EA, Reutelingsperger CPM, Clarke MCH, Bennett MR, Schurgers LJ. Role of medial vascular smooth muscle cell pre-ablation in atherogenesis and plaque vulnerability. International Vascular Biology Meeting (IVBM), June 2018, Helsinki, Finland

Jaminon AMG, van Gorp RH, Bucerius J, Bauwens M, Leenders, P, Floege J, Reutelingsperger CPM, Schurgers LJ. Detection of active mineralisation in vivo with $\mathrm{Na} 18 \mathrm{~F}$ in experimental animals. Nederlandse Klinisch Radiochemische Vereniging (NKRV) meeting, January 2018, Maastricht, The Netherlands.

Jaminon AMG, van Gorp R, Jahnen-Dechent W, Floege J, Reutelingsperger CPM, Schurgers LJ. A Vascular Smooth Muscle Cell Biohybrid High Content Screening Platform for Atherogenicity. International Vascular Biology Meeting (IVBM), November 2016, Boston, USA.

\section{Abstracts}

Dai L, Jaminon AMG, Wasilewski GB, Qureshi AR, Brismar TB, Ripsweden J, Heimbürger O, Barany P, Schurgers LJ, Lindholm B, Stenvinkel P. Poor Vitamin K status is associated with increased mortality in peritoneal dialysis patients. International Society for Peritoneal Dialysis (ISPD), (postponed), Glasgow, Scotland.

van Gorp RH, Jaminon AMG, Baaten CC, Heemskerk JW, Reutelingsperger CP, Spronk HMH, Schurgers LJ. Long-term Vitamin K antagonist treatment induces calcification and atherosclerotic plaque progression, promoting a prethrombotic state. European Atherosclerosis Society (EAS), May 2019, Maastricht, The Netherlands

Mezger STP, Jaminon AMG, Cillero-Pastor B, Schurgers LJ, Mingels AMA, Heeren RMA. Three dimensional MALDI-imaging mass spectrometry mapping of a calcified 
mouse heart. Mass Spectronomy: Application to the Clinic Lab (MSACL), September 2018, Salzburg, Austria.

Jaminon AMG, Willems BAG, van Gorp RH, Recarti C, Schiffers PMH, van Zandvoort M, Biessen EA, Reutelingsperger CPM, Clarke MCH, Bennett MR, Schurgers LJ. Role of medial vascular smooth muscle cell pre-ablation in atherogenesis and plaque vulnerability. International Vascular Biology Meeting (IVBM), June 2018, Helsinki, Finland

van Gorp RH, Jaminon AMG, Baaten CC, Heemskerk JW, Reutelingsperger CP, Spronk HMH, Schurgers LJ. Long-term Vitamin K antagonist treatment induces calcification and atherosclerotic plaque progression, promoting a prethrombotic state. International Vascular Biology Meeting (IVBM), June 2018, Helsinki, Finland.

Jaminon AMG, van Gorp RH, Bucerius J, Bauwens M, Leenders, P, Floege J, Reutelingsperger CPM, Schurgers LJ. Detection of active mineralisation in vivo with $\mathrm{Na} 18 \mathrm{~F}$ in experimental animals. Nederlandse Klinisch Radiochemische Vereniging (NKRV) meeting, January 2018, Maastricht, The Netherlands.

van Gorp RH, Jaminon AMG, Bauwens M, Bucerius J, Brandenburg V, JahnenDechent W, Spronk HMH, Reutelingsperger CP and Schurgers LJ. Oral anticoagulants as modulators of arterial calcification. International Society on Thrombosis and Haemostasis (ISTH) July 2017, Berlin, Germany.

Mezger STP, Jaminon AMG, Schurgers LJ, Mingels AMA, Bekers O, Cillero-Pastor B, Heeren RMA. MALDI-Imaging Mass Spectrometry: studying novel pathophysiological markers in heart failure. International Mass Spectrometry School, July 2017, Dubrovnik, Croatia

Jaminon AMG, van Gorp R, Jahnen-Dechent W, Floege J, Reutelingsperger CPM, Schurgers LJ. A Vascular Smooth Muscle Cell Biohybrid High Content Screening Platform for Atherogenicity. International Vascular Biology Meeting (IVBM), November 2016, Boston, USA.

Chatrou ML, Kusters DHM, Willems BAG, Jaminon AMG, Norling L, Proudfoot D, Spohn G, Spronk HM, Gruell H, Reutelingsperger CPM, Schurgers LJ. Vitamin Kantagonists: a two edged sword. Bi-phasic effect of VKA on atherosclerotic plaque development. 24th International Symposium of Thrombosis and Hemostasis (ISTH), July 2013, Amsterdam, The Netherlands. 


\section{Chapter 13}

Dankwoord 
En dan ben ik nu aangekomen bij het laatste hoofdstuk van dit proefschrift: het dankwoord. Ik weet dat dit het meest gelezen hoofdstuk van mijn proefschrift zal zijn, niet geheel onterecht, want onderzoek doe je niet alleen en er zijn een aantal mensen die ik graag in het zonnetje wil zetten.

Allereerst wil ik mijn promotieteam bedanken: prof. dr. Leon Schurgers en prof. dr. Chris Reutelingsperger.

Mijn promotor prof. dr. Schurgers. Beste Leon, jij bent vanaf het begin mijn directe begeleider geweest en je bijdrage aan dit proefschrift is dan ook bijzonder groot. Ik kan me nog goed ons eerste gesprek herinneren dat plaatsvond tijdens mijn sollicitatie voor een $\mathrm{PhD}$ positie. Er was direct een klik, en we spraken een uur lang over onderzoek, waar we beiden zeer gepassioneerd over waren. Dit gesprek voelde dan ook helemaal niet als een sollicitatiegesprek. Jouw enthousiasme over je eigen werk werkt aanstekelijk en dat heeft mij gedurende mijn promotietraject ook vaak op de been gehouden. Mijn promotietraject heeft immers de nodige tegenslagen gekend. Telkens wanneer ik enigszins gefrustreerd jouw kamer binnen kwam was jij er met het nodige positivisme om me weer de goede richting op te sturen. Daarnaast was jij ook altijd geïnteresseerd in mijn privéleven. (Ik denk dat dit jou als persoon typeert, je bent een man die naast het werk ook iemand privé wil leren kennen). Ik heb de afgelopen jaren heel wat lief en leed met je gedeeld en je hebt het niet nagelaten persoonlijke levenslessen met mij te delen, dit heb ik altijd erg gewaardeerd.. Daarnaast hebben we ook veel gezellige momenten buiten werk beleefd samen. Voor al deze mooie momenten ben ik je zeer dankbaar.

Mijn promotor prof. dr. Reutelingsperger. Beste Chris, hoewel je iets verder van mijn onderzoek af stond ben ik blij met jou als promotor. Ik heb er bewondering voor hoe je altijd weer een verrassende blik hebt bij al het onderzoek dat we doen. Daarnaast vind ik het mooi om te zien hoe jij een wetenschappelijke carrière hebt gecombineerd met die van het bedrijfsleven. Jouw hypothese gedreven vragen waren altijd verhelderend en brachten me altijd terug naar hoofdpunten in plaats van bijzaken. Verder is een van mijn hoogtepunten samen met jou de trip naar Boston, waar ik je ook persoonlijk heb leren kennen, dat was een prachtige ervaring.

I would like to thank the members of the reading committee, prof. dr. Shanahan, prof. dr. Beulens, prof. dr. Kooi, dr. Blankesteijn and prof. dr. De Leeuw for being part of my reading committee and for their critical reading and proficient evaluation of this doctoral thesis. Also I would like to thank all the members of the corona.

Dit proefschrift mag ik verdedigen met twee speciale mensen aan mijn zijde, die veel voor mij en mijn onderzoek hebben betekend.

Lieve Yvonne, het is ondertussen al wat jaartjes geleden dat we elkaar leerden kennen tijdens de afstudeerstage van mijn master. Er was meteen een klik tussen ons 'heuvellanders'. We hebben vanaf toen een hechte band ontwikkeld waarin we lief en leed met elkaar hebben gedeeld. Eerst als directe collega, vervolgens als collega op de UM en nu als goede vrienden. Samen met Joël en jullie dochtertje Gisèle hebben we al veel mooie momenten beleefd. En als er belangrijke momenten in ons leven zijn waar je 
niet fysiek bij aanwezig bent, dan zorg je dat er altijd een kaartje wordt gestuurd. Ik moet zeggen dat de universiteit anders is nu je er niet meer bent maar ik ben enorm blij dat je mijn paranimf wilt zijn en ik weet zeker dat we nog vele bijzondere momenten met elkaar zullen delen. Ik wil je bedanken voor alle steun die je me hebt gegeven en ik wens je een mooi vervolg van je carrière toe!

Beste Rick, we zijn tegelijkertijd begonnen bij Leon als $\mathrm{PhD}$ student. Het is voor mij niet meer dan logisch dat je mijn paranimf bent want ik denk dat we heel veel aan elkaar hebben gehad tijdens ons promotie traject. Ik weet nog goed dat we als twee broekies begonnen en we samen de "legacy" van de alfa-mannetjes die voor ons promoveerden moesten voortzetten. Terugkijkende denk ik dat we daar goed in zijn geslaagd en ondertussen zijn we meer vrienden dan collega's geworden. Ik weet dat we ondertussen ook genoeg bijnamen hebben gekregen. We worden gezien als die twee van Leon of de gebroeders van Leon. Ik moet zeggen dat ik het nu ook wel zo zie: we hebben elkaar geholpen waar nodig en hebben veel met elkaar gedeeld. Zowel op onze werkkamer als iemand het nodig had om even te spuien of in het lab waar we elkaar veel geholpen hebben met de dierenexperimenten. Wat ik enorm kan waarderen is jouw Brabantse nuchter- en eerlijkheid. Je was een geweldige $\mathrm{PhD}$ collega en ik hoop dan ook dat je een mooie nieuwe uitdaging vindt voor in de toekomst.

Naast mijn promotoren is er een vaste kern binnen onze groep die het onderzoek draaiende houdt, en die ik ook graag wil bedanken.

Lieve Cécile, ik heb je wel vaker de moeder van onze groep genoemd en dat is denk ik ook de perfecte beschrijving voor jou. Je bent naast een betrouwbare kracht ook iemand die de gezelligheid in de groep brengt, iemand die altijd wel in is voor een kopje koffie en een goed gesprek. Ik vond het altijd fijn om mijn hart bij je te kunnen luchten.

Lieve Petra, jij bent het manusje van alles. Ik ken denk ik niemand die zo veelzijdig is als jij. Iedereen, maar dan ook letterlijk iedereen komt naar jou toe met vragen die je zonder problemen oplost. Naast de werk gerelateerde zaken hebben we vaak privé gesprekken gehad die ik enorm kon waarderen.

Beste Niko, de gekke Belg van het stel. Voor jou heb ik denk ik de meeste bijnamen bedacht die ik hier maar niet zal noemen (hè klinge). Jij bent iemand die altijd wel in is voor een babbel en brengt enthousiasme en lol in de groep. Verder kan ik me de talrijke dinertjes met overnachting bij mij thuis nog goed herinneren, waarbij we vaak hebben genoten van een laat biertje buiten in de tuin.

Beste Liset, ik wil je bedanken voor de fijne tijd die we hebben gehad. We hebben wat minder persoonlijk contact gehad maar je staat altijd open voor een inhoudelijke discussie om de talrijke problemen die onderzoek soms oplevert te kunnen ophelderen.

Beste Peter, pas zeer recent toegevoegd aan de vaste kern. Jij als dierdeskundige hebt een zeer grote bijdrage geleverd aan mijn boekje. Ik waardeer het enorm hoeveel kundige hulp je hebt geboden met alle dierproeven. Verder ben je een sociaal persoon die super 
betrouwbaar is. Dat is zeker gebleken tijdens de corona crisis waarin je met veel passie de dierproeven hebt doorgezet.

Our group has grown since I started my $\mathrm{PhD}$ and has become a very multicultural one.

Dear Ploi, my other roommate. I can still remember the countless attempts to pronounce your name. You are a very polite and cheerful lady and I liked the numerous discussions about the fruits and other habits of Thailand. I wish you all the best in finishing your $\mathrm{PhD}$ and for your future.

Dear Gosia, Greg, Angelina, Nikolas, Cengiz, Rogier and lately Anxhela and Vanessa. Thank you for being part of our amazing group and being helpful whenever needed. I wish you all the best for the future and keep the spirit going.

Naast onze groep is de afdeling biochemie erg divers en wil ik ook hiervan een aantal mensen speciaal bedanken.

Beste Stijn, Danique en Annemiek. De vaste leden van het koffie-drink-groepje. Ik wil jullie bedanken voor de talloze gezellige gesprekken tijdens onze koffiemomentjes. Jullie kwamen altijd enthousiast vragen of ik mee koffie ging drinken en ik moet zeggen dat dit soms een welkome afleiding was van het werk. Met jullie kon ik ook de minder leuke zaken bespreken die horen bij een promotietraject. Het was fijn dat jullie hier altijd een luisterend oor voor hadden. Verder kan ik me nog goed de vele gezellige activiteiten herinneren die jullie organiseerden en die onze afdeling zo speciaal maakt. Stijn: jou als buurman wil ik graag nog even apart bedanken, omdat je het mogelijk maakte dat Famke en ik het afgelopen jaar af en toe een weekend weg konden gaan, door onze vaste konijnenverzorger te zijn!

All other PhD students of Biochemistry, thank you for all the nice moments we shared. Ik wil hier een aantal personen nog kort uitlichten:

Jens, wat een sympathiek persoon ben je toch. Ik waardeer de gesprekken die we hebben gevoerd over zowel wetenschappelijke als privé aangelegenheden.

Mieke, jou wil ik bedanken voor de leuke en diverse gang gesprekken die we hebben gehad. Ik wens je veel geluk in je nieuwe huis in Eijsden.

Stan, bedankt voor het organiseren voor de bier en wijnproeverijen, het brengt verbondenheid op de afdeling.

Billy, je hebt een afwijkend pad bewandeld maar uiteindelijk ben je (gedeeltelijk) teruggekeerd naar het oude nest. Ik wil je bedanken voor de leuke gesprekken tijdens en na het werk. Ik herinner me nog goed de Star Wars avonden samen met Rick, met de nodige biertjes, die erg gezellig waren.

Verder wil ik alle PI's en andere ervaren onderzoekers bedanken: Hugo, Johan, Tilman, Judith, Gerry, Betta, Henri, Rene, Marijke, Dennis, Hans, Kanin, Rory en Ingrid. Ik heb veel van jullie geleerd de afgelopen periode.

Paola, jou wil ik apart bedanken. Allereerst sorry voor de vele onderbrekingen tijdens je werk omdat ik Niko weer eens wat moest vragen. Verder hebben we veel leuke 
gesprekken gehad over zowel privé als werk gerelateerde zaken, je was altijd geïnteresseerd in hoe het met mij ging.

Stella, jij mag natuurlijk niet ontbreken in dit dankwoord. Jij bent de echte gangmaker van de afdeling biochemie en ik krijg nog steeds een lach op mijn gezicht als ik denk aan de vele Bicky burgers die je hebt gebakken.

Beste Trees en Lidewij, jullie zijn de motor van de afdeling. Met de administratieve rompslomp kon ik altijd bij jullie terecht, altijd behulpzaam en open voor een leuk gesprek. Ik wil jullie dan ook bedanken voor de talloze keren dat jullie me hebben geholpen.

Tom M, Magdi, Constance, Frauke, Peter, Daniëlle B, Tanja en Roy, de oudere garde van biochemie. De meesten van jullie zijn ondertussen vertrokken. Ik wil jullie bedanken voor de vele leuke momenten die we hebben gehad binnen de afdeling.

Alle studenten die me hebben geholpen tijdens mijn promotie traject: Katrien, Demi, Renée, Maurice, Nicole, Maria en Bastiaan. Dank voor jullie bijdrage aan dit proefschrift.

Maurice, ik vond het heel erg leuk om jou te zien groeien tijdens je master stage in onze groep. Het heeft uiteindelijk succes gebracht en we hebben tot op de dag van vandaag nog steeds een leuke samenwerking. Ik hoop dat we deze ook kunnen blijven behouden. Nicole, your a special one. I can still remember the day you came to our lab, a little bit clumsy but with the heart on the right place. I am proud that you did such a great job in our lab and look at you now, you are an amazing independent researcher.

Beste Martijn, Dennis K en Brecht jullie wil ik ook bedanken. Jullie hebben ervoor gezorgd dat de fundering van het verkalkingsonderzoek is gelegd, hier heb ik verder op kunnen bouwen.

Dan kom ik nu bij de collega's van de UM.

Beste Jacques, Agnieska, Helma en Nicole. Heel erg bedankt voor het meedenken en werken aan de dierproeven. Jullie worden als technici vaak onderschat maar ik waardeer het enorm dat jullie met me mee hebben gedacht.

Beste Frederique, we hebben elkaar tijdens het uitvoeren van de CATAPuLT studie goed leren kennen, je bent een sociale en zeer gestructureerde collega. Ik ben blij dat we vele discussies over verkalking hebben mogen voeren. Ondertussen ben je al gepromoveerd en ben je in opleiding tot cardioloog, chapeau!

Dear Luise, thank you for helping me with the DT project. I wish you all the best in finishing your PhD.

Dr. Natour en dr. Mees. Beste Ehsan en Barend, veel dank voor al het weefsel dat we vanuit de kliniek krijgen om onze gladde spiercellen uit te isoleren. De open hart operatie die dr. Natour uitvoerde en waarbij ik aanwezig mocht zijn zal me altijd bijblijven, een indrukwekkend staaltje vaardigheid. Barend, ik verheug me op onze intensievere 
samenwerking de komende jaren, ik hoop dat we veel interessante ontdekkingen zullen gaan doen!

Kim, Margaux en Tom H. Ik wil jullie bedanken voor de leuke samenwerkingen en de hulp die jullie me hebben geboden.

Dr. Neumann. Dear Dietbert, I want to thank for the opportunity to start in Maastricht as a master student and to work for you as a researcher at the department of Molecular Genetics. You have been a mentor for me before I started my PhD, thank you for that. Van de afdeling MolGen wil ik in het bijzonder prof. Glatz en Petra bedanken, ook omdat jullie je hebben ingezet om mij na mijn afstudeerstage op de UM als onderzoeker te behouden.

Naast collega's, wil ik ook al mijn vrienden en familie bedanken. Niet alleen door de interesse in mijn werk maar vooral omdat ze zorgen voor de nodige ontspanning, om buiten het werk ook af te kunnen schakelen. Er zijn een aantal mensen die ik in het bijzonder wil bedanken:

De Bonte Bruij, als vriendengroep hebben we allemaal een connectie met de harmonie van Vijlen. Ondertussen is de groep flink gegroeid en zijn er partners en kinderen bijgekomen: we zijn een echt bont gezelschap zo met z'n allen. Ik wil jullie bedanken voor de vele ontspannen momenten als we met vrienden onder elkaar zijn, zoals bieravonden met de mannen, bioscoop avonden met de filmliefhebbers en etentjes met de sushi liefhebbers. En in het bijzonder de vele Bonte Bruij weekenden. Het is fijn om op deze vriendschap terug te kunnen vallen.

Nils, ook deel van de Bonte Bruij, maar ik wil je toch nog even apart noemen. Je hebt altijd contact met mij gehouden gedurende mijn tijd in Leiden en sinds ik terug ben is ons contact veel closer geworden. Ik ben dan ook oprecht trots dat jij de Peetoom bent van onze dochter Marit, en ik weet nu al zeker dat dit de juiste keuze was. Als ik jou hier noem mag Lonneke natuurlijk niet ontbreken, we hebben met z'n vieren al moeilijke periodes met elkaar gedeeld maar we weten dat we op elkaar kunnen bouwen. Ik vind onze vriendschap erg belangrijk en speciaal.

Robbin, jij als 'oudste' vriend mag natuurlijk niet ontbreken. Je was ook niet voor niets getuige op mijn bruiloft. De vele discussies over voetbal, formule 1 en wielrennen, live of via de app helpen mij echt om te ontspannen. Ook onze wekelijkse rondjes op de racefiets hebben me altijd goed gedaan. Dankjewel voor je vriendschap, en dit geldt natuurlijk ook voor Vera. Ik hoop dat we samen nog veel leuke momenten mogen beleven.

Maurice en Michelle, mijn broer en zusje. We zijn samen opgegroeid in Vijlen en hebben met elkaar ook veel meegemaakt. Ik ben blij dat jullie er zijn. Jullie hebben op geheel eigen wijze jullie eigen succes bereikt en dat is iets om trots op te zijn.

Josien, Cyriel, Twan en Jorrit, mijn schoonfamilie. Ik ben blij dat jullie in mijn leven zijn. We delen lief en leed met elkaar en het is zo leuk en uniek om de jongens te zien 
opgroeien. Ik ben trots dat ik hun 'nonk Armand' mag zijn. Ik hoop dat we nog veel leuke momenten mogen hebben en dat we nog vaak samen kunnen genieten.

Evelyn en Jo, mijn schoonouders. Sinds ik met Famke samen ben heb ik het gevoel dat ik er twee ouders bij heb gekregen. Ik kan me nog goed herinneren dat ik de eerste keer bij jullie op de bank zat, alweer flink wat jaartjes geleden. Jullie hebben me toen met open armen ontvangen en doen dat nog steeds. Ik waardeer het enorm hoeveel jullie voor mij en Famke betekenen. Jullie staan altijd voor ons klaar en willen ook graag leuke momenten met ons delen. Ik hoop dan ook dat er nog vele zullen volgen.

Maria, lieve mam. Hoewel je op dit moment niet meer alles volledig meekrijgt weet ik dat je trots op me bent om wat ik heb bereikt. Vroeger heb je altijd goed voor ons gezorgd, we kwamen niks te kort. Op dit moment moet ik helaas voor jou zorgen en hoewel je je niet meer zo goed kunt uiten weet ik dat je dit diep van binnen erg waardeert. Ook weet ik dat nu Marit, je kleindochter er is, je hernieuwde energie hebt, ik zie je steeds stralen als je haar ziet. Mam bedankt voor alles wat je voor me hebt gedaan.

Pierre, beste pap. Ook jou wil ik bedanken voor de goede zorgen die je ons hebt gegeven. We hebben niet de makkelijkste jaren achter de rug maar dat gaat inmiddels stukken beter. Je bent altijd bereid om me te komen helpen wanneer ik een handje hulp in huis kan gebruiken. Ik merk dat we als team ook steeds vaardiger worden en de meeste problemen samen kunnen oplossen. Nu Marit er is heb je ook een nieuwe rol in je leven, namelijk die van opa, ik vind het mooi om te zien hoeveel plezier je hier in hebt. Pap ik wil je bedanken dat je altijd voor me klaarstaat.

En dan de twee belangrijkste personen in mijn leven. Famke, mijn lieve sterke vrouw, mijn steun en toeverlaat. Jou moet ik toch het allermeeste van iedereen bedanken. Je hebt mij altijd gesteund gedurende het promotie traject. Ik weet dat het uiteindelijk een lange zit is geworden maar je bent altijd achter me blijven staan en hebt me gestimuleerd om verder te gaan op de momenten dat het niet opschoot. Wat hebben we ondertussen toch al veel meegemaakt in de 12 jaar dat we samen zijn. De vele mooie vakanties, onze prachtige bruiloft en recent de geboorte van onze dochter Marit. Ik kan niet meer dan super trots op je zijn als ik zie hoe jij je ondertussen ook hebt ontwikkeld, van jonge geneeskunde student, tot volwassen dokter, (bijna) huisarts en lieve moeder van ons kleine meisje. Jouw ondersteuning, zorgzaamheid maar ook je pittige karakter waardeer ik enorm, het houdt me scherp. Je weet dat ik zielsveel van je hou en ik hoop dan ook dat we nog veel mooie momenten samen mogen meemaken. Marit, mijn lieve dochter. Alle clichés zijn waar, jij bent het mooiste wat me is overkomen. Hoewel jij een hele moeilijk start hebt gehad heb je je daar ontzettend goed doorheen geslagen en daar heb ik heel veel bewondering voor. Jij hebt laten zien over de echte koempelmentaliteit te beschikken en mij als geen ander laten zien wat je kunt bereiken met een portie doorzettingsvermogen en vechtlust. $\mathrm{Nu}$ we samen thuis zijn, zie ik hoe snel je je ontwikkelt en wat voor een vrolijke dame je al bent. Weet dat ik heel veel van je hou en dat ik dat voor altijd zal blijven doen. 
